\title{
Equivariant Seiberg-Witten Floer Homology
}

\author{
Matilde MARCOLli AND BAI-LING WANG
}

In this paper we construct, for all compact oriented threemanifolds, a $U(1)$-equivariant version of Seiberg-Witten Floer homology, which is invariant under the choice of metric and perturbation. We give a detailed analysis of the boundary structure of the monopole moduli spaces, compactified to smooth manifolds with corners. The proof of the independence of metric and perturbation is then obtained via an analysis of all the relevant obstruction bundles and sections, and the corresponding gluing theorems. The paper also contains a discussion of the chamber structure for the Seiberg-Witten invariant for rational homology 3-spheres, and proofs of the wall crossing formula, obtained by studying the exact sequences relating the equivariant and the non-equivariant Floer homologies and by a local model at the reducible monopole.

\section{Contents.}

$1 \quad$ Introduction . . . . . . . . . . . . . . 452

1.1 Summary of the main results . . . . . . . . . 453

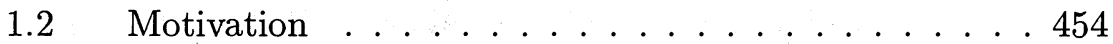

1.3 Outline of the paper . . . . . . . . . . 456

2 Non-equivariant Floer theory . . . . . . . . . . . . . . 462

2.1 Three dimensional Seiberg-Witten theory . . . . . . 462

2.2 Chamber structure . . . . . . . . . . . . 467

2.3 Perturbation of flow lines . . . . . . . . . . 472

2.4 Transversality of $\mathcal{M}(a, b) \ldots \ldots 77$

2.5 Floer homology . . . . . . . . . . . . . . . 483

3 Morse-Bott theory . . . . . . . . . . . . . . 486

$3.1 \quad$ Framed moduli space . . . . . . . . . . . . 487

3.2 Gradient flow lines . . . . . . . . . . . . . . . . 490

3.3 Relative Morse Index . . . . . . . . . . . . . . . . 492

3.4 Decay estimate . . . . . . . . . . . . . . . 494

3.5 Transversality of $\mathcal{M}\left(O_{a}, O_{b}\right) \ldots \ldots \ldots 01$

4 Boundary structure . . . . . . . . . . . . . 501

4.1 Convergence theorem . . . . . . . . . . 503 
4.2 Gluing theorem . . . . . . . . . . . . . . . . 512

4.3 Multiple gluing theorem and corner structure . . . . . 541

5 Equivariant Homology . . . . . . . . . . . . . . . . . . 549

5.1 The equivariant complex . . . . . . . . . . . 550

5.2 Equivariant Floer Homology _. . . . . . . . . . . 551

$5.3 \quad$ Intermezzo: the coefficient $m_{a c} \ldots \ldots \ldots \ldots 55$

6 Topological invariance . . . . . . . . . . . . . 563

6.1 The boundary structure of $\mathcal{M}\left(O_{a}, O_{a^{\prime}}\right) \ldots \ldots 571$

6.2 Obstruction bundle and gluing theorems . . . . . . 584

6.3 Proof of topological invariance . . . . . . . . . 600

$7 \quad$ Wall crossing formula for the Casson-type invariant . . . . . . 614

7.1 Comparison with the non-equivariant Floer Homology 615

7.2 Wall-crossing formula: the algebraic picture . . . . 622

7.3 Wall-crossing formula: the geometric picture . . . . 628

\section{Introduction.}

und das hat mit ihrem Singen

die Loreley getan.

(H. Heine)

The dimensional reduction of the Seiberg-Witten equations on 4manifolds leads to equations on a compact oriented 3-manifold $Y$, obtained by considering translation invariant solutions of the original equations in a temporal gauge on an infinite cylinder $Y \times \mathbb{R}$ with translation invariant metric. The solutions modulo gauge transformations of the reduced equations on $Y$ can be regarded as the critical points of a Chern-Simons-Dirac functional, defined on the configuration space of $U(1)$-connections and spinors. There is an associated Floer-type homology, which depends on the choice of the $\operatorname{Spin}_{c}$-structure. Some of the properties of this Seiberg-Witten Floer homology have been discussed in [11], [13], [27], [38], [40], [41], [42], [54], [63], [64], [65]. The properties are different according to whether the manifold $Y$ is a rational homology sphere or a manifold with $b^{1}(Y)>0$. In particular, if $Y$ is a rational homology sphere, for any $\operatorname{Spin}_{c}$-structure there is always a reducible point in the moduli space, namely solutions with vanishing spinor and a non-trivial stabilizer of the gauge group action. Unlike the case with non-zero Betti number, the reducible cannot be perturbed away just by adding a co-closed 1 -form to the curvature equation. This gives rise to an interesting metric dependence phenomenon, which has no analog in 
the instanton case. The Seiberg-Witten Floer homology for manifolds with $b^{1}(Y)>0$ presents a periodicity of the grading and is graded over the integers only after passing to a cover. With the formulation we follow in this paper, it is no longer finitely generated. Unlike the case of $b^{1}(Y)=0$, the Floer homology of 3-manifolds with $b^{1}(Y)>1$ is independent of the metric and perturbation, and, since we are dealing only with the case of $\operatorname{Spin}_{c^{-}}$ structures with $c_{1}(L) \neq 0$, and with perturbations that are cohomologically trivial, this is true for $b^{1}(Y)=1$ as well.

Summarizing, there are Seiberg-Witten Floer homologies with substantially different properties depending on the underlying three-manifold $Y$. One purpose of this paper is to give a unifying approach and a construction of Seiberg-Witten Floer homology for all 3-manifolds, which is always independent of metric and perturbation.

The Floer homology always depends on a choice of the $\operatorname{Spin}_{c}$-structure, as does the construction of Seiberg-Witten invariants. Thus, we really obtain, over a 3-manifold $Y$, a family of Floer homologies $H F_{*}^{S W}(Y, \mathbf{s})$, param-

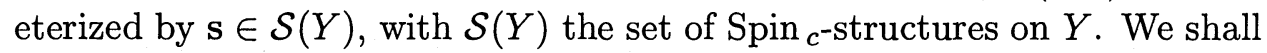
always consider a fixed $\operatorname{Spin}_{c}$-structure, hence, for simplicity of notation, we never explicitly mention the $\mathbf{s}$ dependence.

\subsection{Summary of the main results.}

We give a unifying approach to Seiberg-Witten Floer theory, by introducing a version of Seiberg-Witten Floer homology that is defined for all threemanifolds and is always metric independent. In the case of manifolds with $b^{1}(Y)>0$ we shall only consider $\operatorname{Spin}_{c^{-}}$structures with $c_{1}(L) \neq 0$. The case $b^{1}(Y)>0$ and $c_{1}(L)=0$ has a more subtle behavior with respect to the choice of the perturbation and the compactification of the moduli spaces of flow lines (cf. Remark 2.18). Since this case arises in important problems connected to surgery formulae, we consider it separately in [42]. We construct an equivariant version of the Seiberg-Witten Floer homology. In the case of rational homology spheres, this approach counts all the contributions, both from the reducible point and from the irreducibles. The equivariant Floer homology also depends on the choice of the Spin $c^{- \text {structure. }}$ We prove the invariance of the equivariant Floer homology with respect to the metric and perturbation. The argument involves Taubes' obstruction bundle technique and an appropriate gluing theorem. In the two distinct cases of rational homology spheres and of manifolds with non-trivial Betti number, we compare the equivariant theory with the constructions of nonequivariant Seiberg-Witten Floer theories. In the case of manifolds with 
non-trivial Betti number, we obtain an isomorphism of the equivariant and the non-equivariant theories (both considered for a fixed $\operatorname{Spin}_{c}$-structure) and in the case of rational homology spheres we construct some exact sequences that relate the equivariant and the non-equivariant theory. The results are obtained by means of the spectral sequences associated to a filtration of the complexes. The metric independence of the equivariant Floer homology together with the exact sequences lead to an algebraic proof of the wall crossing formula for the associated Casson-type invariant. We also provide a geometric proof of the same formula, through the analysis of the local structure of the moduli spaces.

Main Theorem. Let $Y$ be any closed and oriented 3-manifold with a fixed Spin $_{c}$-structure $\mathbf{s}$, with $c_{1}(\mathbf{s})$ non-torsion if $b_{1}(Y)>0$. Then there exists a $U(1)$-equivariant Seiberg-Witten-Floer homology $H F_{*, U(1)}^{S W}(Y, \mathbf{s})$, which is a graded $\mathbb{R}[\Omega]$-module, and is a topological invariant of $(Y, \mathbf{s})$. This Floer homology has the following properties:

- For $b_{1}(Y)>0$ and $c_{1}(\mathbf{s})$ non-torsion, $H F_{*, U(1)}^{S W}(Y, \mathbf{s})$ is finitely generated and isomorphic to the non-equivariant Seiberg-Witten-Floer homology $\mathrm{HF}_{*}^{S W}(Y, \mathbf{s})$.

- For Y a rational homology 3-sphere, and for any fixed choice of metric and perturbation, there exists a long exact sequence relating $H F_{*, U(1)}^{S W}(Y, \mathbf{s})$ and $H F_{*}^{S W}(Y, \mathbf{s})$, of the form

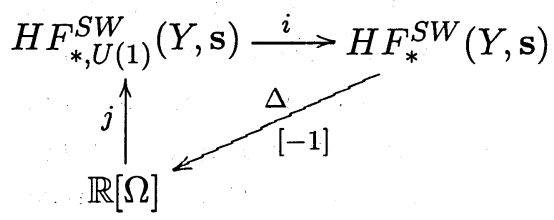

where $\Omega$ is a generator of degree 2 , the maps $i$ and $j$ preserve the grading, and $\Delta$ decreases the grading by one.

\subsection{Motivation.}

Floer homology, as a gauge theoretic invariant of three-manifolds, made its first appearance in the seminal work of Floer [24] on what is now referred to as the Yang-Mills, or instanton, Floer homology. Instanton Floer homology came to play an essential conceptual role in Atiyah's Topological Quantum Field Theory formulation of Donaldson theory [1], and in the definition of 
relative Donaldson invariants of 4-manifolds with boundary and their gluing formulae, see for instance the work of Taubes [62]. Soon after the introduction of the new Seiberg-Witten gauge theory, it became clear that an analogue of Floer homology existed in the Seiberg-Witten context. Nonequivariant versions of Seiberg-Witten Floer theory were presented in the work of K. Iga, M. Marcolli, B.L. Wang, and R.G. Wang. In the last few years, there has been a number of significant papers using Seiberg-Witten Floer homology and gluing theorems for Seiberg-Witten invariants. It is now known (see e.g., [29]) that a good understanding of the relation between the instanton and the Seiberg-Witten Floer homology can lead to very striking topological consequences. However, in the literature (both in the instanton and in the Seiberg-Witten context) there seems to be a fundamental need for a satisfactory foundational work which presents a detailed and careful construction of the Floer homology. It is important to mention that, perhaps surprisingly, the technical aspects involved in the Seiberg-Witten gauge theory are substantially different from the Yang-Mills case and require different techniques. An important issue, which has no analogue in the Yang-Mills case and to which much of this paper is dedicated, is the metric dependence of the Seiberg-Witten Floer homology in the case of homology 3-spheres. Providing a metric independent version of Floer homology is essential in all the important applications which involve surgery formulae and gluing of relative invariants. Along the lines of the theory Austin and Braam [6] developed for instanton homology, we develop an equivariant version of Seiberg-Witten Floer homology. The main result in the paper consists of showing that this is precisely what is needed to the purpose of avoiding the metric dependence problem. The main technical issues involved center around the fine structure of the compactification of the moduli spaces of flow lines of the Chern-Simons-Dirac functional, and around the use of Taubes' obstruction bundles in the course of the proof of the topological invariance of the equivariant Seiberg-Witten Floer homology.

It is not easy to give the skeptical reader an introduction to this paper that justifies the many pages to come. To be perfectly honest, it took a long time to convince ourselves as well of the real need to unravel all the detailed technical issues that appear in the corpus of this paper. Nonetheless, we hope the introductory paragraph above gives sufficiently clear an idea of why it is genuinely useful to write a paper that covers the material presented here. This paper has been under preparation since 1995. It circulated in preprint versions in 1996, 1997, and 2000. Regrettably, several years passed between the time when this paper was submitted and the time when the last of its long series of avatars would finally see the light in printed form incarnation. 
We should signal to the reader the appearance, in the meanwhile, of other papers that have a more or less extended overlap with the present one, most notably [27] and [65].

We summarize, in the rest of the Introduction, the main results of the various sections of this paper, pointing to precise references to Theorems, Sections, etc. where the various statements are presented and proved.

\subsection{Outline of the paper.}

The paper is organized as follows. Section 2 presents preliminary material, starting, in Propositions 2.3, 2.4, and 2.5, with a brief account of SeibergWitten gauge theory on three-manifolds and a brief overview of the properties and the local structure of the moduli space $\mathcal{M}$ of critical points of the Chern-Simons-Dirac functional, and then continuing in Sections 2.3 and 2.4 with an account of the properties of the moduli spaces $\mathcal{M}(a, b)$ of flow lines connecting critical points. These are the moduli spaces that appear in the construction of the non-equivariant versions of Seiberg-Witten Floer theory, as used for instance in [13], [38], [63], [64]. The main result in Sections 2.3 and 2.4 is the construction of a suitable class of perturbations of the flow lines equations, in Definition 2.10, and the proof of transversality in Proposition 2.14. We also discuss the notion of relative Morse index of critical points of the Chern-Simons-Dirac functional in Proposition 2.12 and the orientation of the moduli spaces $\mathcal{M}(a, b)$ in Proposition 2.15. The construction and properties of the non-equivariant Seiberg-Witten Floer theory are briefly summarized in Section 2.5, the main result being the gluing formula of Lemma 2.16, which is essential in establishing the property $\partial \circ \partial=0$ for the boundary of the Floer complex. We do not present a complete treatment of the non-equivariant Floer homology, since all the necessary results follow from the equivariant case of Sections 4, 5, and 6, and the comparison results of Section 7. However, we discuss some essential aspects of the non-equivariant theory, for instance the phenomenon of metric dependence in the case of homology spheres. In the proof of Theorem 3.4 it is shown that, in the case of homology spheres, the unique reducible point is isolated whenever the metric and perturbation are chosen so that the twisted Dirac operator has trivial kernel. This condition on the choice of metric and perturbation determines a chamber structure with codimension one walls which is analyzed in Section 2.2. We prove in Theorem 2.7 that the condition on the Dirac operator is generic and that the space of metrics and perturbations breaks into chambers with codimension one walls. We analyze the structure of the walls in Theorem 2.8. 
In Section 3 we introduce the framed moduli space $\mathcal{M}^{0}$ in the framed configuration space $\mathcal{B}^{0}$. The results of Lemma 3.2, Lemma 3.3, and Theorem 3.4 can be summarized as follows: the framed moduli space $\mathcal{M}^{0}$ is a smooth manifold with a $U(1)$-action: the action is free in the case of manifolds with $b^{1}(Y)>0$. When $b^{1}(Y)=0$, there is a unique fixed point in $\mathcal{M}^{0}$, which corresponds to the reducible point in $\mathcal{M}$. In the case of rational homology spheres, the finitely many inequivalent flat $U(1)$ connections correspond to the unique fixed point in $\mathcal{M}^{0}$ for all the different possible choices of the Spin $_{c}$-structure. In Theorem 3.4 we also show that the critical orbits are all isolated and the Hessian is non-degenerate in the directions orthogonal to the orbits. That is, the Chern-Simons-Dirac functional satisfies the MorseBott condition.

In the remaining of Section 3 we analyze the moduli spaces $\mathcal{M}\left(O_{a}, O_{b}\right)$ of gradient flow lines connecting critical orbits $O_{a}$ and $O_{b}$. In Section 3.3, Theorem 3.8, we discuss the relative Morse index of critical orbits, which requires introducing the relevant Fredholm theory. The analysis of Section 3.4 is aimed at proving that all finite energy solutions of the Seiberg-Witten equations on the manifold $Y \times \mathbb{R}$ decay asymptotically to critical orbits, with an exponential weight which is determined by the smallest absolute value of the non-trivial eigenvalues of the Hessian. Thus, every finite energy solution lies in some moduli space $\mathcal{M}\left(O_{a}, O_{b}\right)$. The exponential decay to the endpoints of solutions in a moduli space $\mathcal{M}\left(O_{a}, O_{b}\right)$ is proved in Theorem 3.12 , using the result of Lemma 3.10 (a Palais-Smale condition), and the estimate of Lemma 3.13. The argument of Theorem 3.12 is basically a "finite energy implies finite length" type result (cf. [58]). Finally, we give the transversality result for the moduli spaces $\mathcal{M}\left(O_{a}, O_{b}\right)$ in Proposition 3.14 .

Section 4 is dedicated to the existence and properties of the compactification of the moduli spaces $\hat{\mathcal{M}}\left(O_{a}, O_{b}\right)$ of unparameterized flow lines. We prove that a compactification can be obtained by adding boundary strata of broken trajectories. The codimension $\mathrm{k}$ strata in the boundary are of the form

$$
\bigcup_{c_{1}, \cdots c_{k}} \hat{\mathcal{M}}\left(O_{a}, O_{c_{1}}\right) \times_{O_{c_{1}}} \hat{\mathcal{M}}\left(O_{c_{1}}, O_{c_{2}}\right) \times \cdots \times_{O_{c_{k}}} \hat{\mathcal{M}}\left(O_{c_{k}}, O_{b}\right)
$$

where the union is over all possible sequences of critical points $c_{1}, \cdots c_{k}$ with decreasing indices. We also show that the compactification has a fine structure of a smooth manifold with corners. The analysis in Section 4.1 shows that a compactification exists, and that the points in the ideal boundary consist of a certain subset of the set (1) of broken trajectories through inter- 
mediate critical points. This is the main result of Theorem 4.1. Unlike other problems of compactification in gauge theory, the construction of Floer homology requires a more detailed analysis of the compactification. In fact, the property that the boundary $D$ of the (equivariant) Floer complex satisfies $D \circ D=0$ requires non only to know that a compactification of the moduli spaces $\hat{\mathcal{M}}\left(O_{a}, O_{b}\right)$ exists, but also that all the broken trajectories (1) in the ideal boundary occur in the actual boundary of the compactification. Moreover, since the argument in the proof of $D \circ D=0$ is based on a version of Stokes' theorem for manifolds with corners, it is essential to analyze the fine structure of the compactification at the boundary strata (1). At the end of Section 4.1, in Remark 4.5 we also compare different existing definitions of the Seiberg-Witten Floer homology for manifolds with $b^{1}(Y)>0$.

In Section 4.2 we prove that in fact all broken trajectories through intermediate critical points listed in (1) actually occur in the compactification, the main result being the gluing theorem 4.9. This theorem only deals with the codimension one boundary, namely with the trajectories that break through one intermediate critical orbit. The gluing construction which proves Theorem 4.9 involves several technical aspects. The main idea is to introduce a pre-gluing procedure, in which an approximate solution to the Seiberg-Witten equations is obtained by splicing together with cutoff functions a pair of solutions in the fibered product

$$
\hat{\mathcal{M}}\left(O_{a}, O_{b}\right) \times_{O_{b}} \hat{\mathcal{M}}\left(O_{b}, O_{c}\right) .
$$

The gluing construction then takes place by proving, via a fixed point argument (Remark 4.20), that close enough to the approximate solution there is a unique actual solution in $\hat{\mathcal{M}}\left(O_{a}, O_{c}\right)$. The central technical issues connected to this argument revolve around the analysis of the eigenspace of small eigenvalues of the linearization of the Seiberg-Witten equations at the approximate solutions. Lemma 4.7 and Lemma 4.8 deal with the slices of the gauge action. These are necessary in order to introduce the pre-gluing construction in Lemma 4.10. The analysis of the small eigenvalues is developed essentially in Lemma 4.12, Lemma 4.13, and Lemma 4.15, and then recalled, in Section 6, in Lemma 6.13. In Lemma 4.15 we also provide an estimate of the rate of decay of the small eigenvalues. The eigenspaces of small eigenvalues, which give the normal bundle for the gluing construction, are introduced in Definition 4.16 and in Proposition 4.17, cf. also Corollary 4.18 on the splitting of the index, and Remark 4.22.

In Section 4.3 we extend the results of Section 4.2 , in order to deal with the strata of higher codimension in the compactification, and to show the 
fine structure of the compactified moduli spaces, namely the fact that the moduli spaces $\hat{\mathcal{M}}\left(O_{a}, O_{b}\right)$ compactify to smooth manifolds with corners, in the sense of Melrose [44]. The main result on the corner structure, Theorem 4.23, is based on Proposition 4.25, which is an inductive generalization of Theorem 4.9, and shows the existence of a smooth atlas of charts with corners.

Throughout all the gluing construction in Section 4, we make essential use of the transversality result, namely of the fact that the linearizations at the solutions in $\hat{\mathcal{M}}\left(O_{a}, O_{b}\right)$ and $\hat{\mathcal{M}}\left(O_{b}, O_{c}\right)$ have trivial cokernel. We say in such cases that the gluing is "unobstructed". The spectral analysis in Lemma 4.12, Lemma 4.13, and Lemma 4.15, however, is formulated in more general terms that adapt to the case where cokernels are present. This gluing theory "with obstructions" is elaborated in Section 6, with the purpose of proving the topological invariance of the equivariant Floer homology.

As we discuss in the beginning of Section 4, the fine structure of the compactification of the moduli spaces of flow lines is necessary in order to establish the existence of the Floer complex, namely the fact that the boundary operator $D$ of the equivariant Floer complex satisfies $D^{2}=0$.

Section 5 introduces the equivariant complex, the boundary operator, and the equivariant Floer homology. With the essential use of the results of Section 4, we prove the property $D^{2}=0$ in Theorem 5.1. We then give an explicit description of the boundary operator of the equivariant Floer complex in Proposition 5.3.

Section 6 contains the proof of the invariance of the equivariant SeibergWitten Floer homology with respect to the choice of the metric and perturbation. The proof of the invariance is obtained by defining a chain map $I$ connecting the equivariant Floer complexes associated to choices $\left(g_{0}, \nu_{0}\right)$ and $\left(g_{1}, \nu_{1}\right)$, a similar chain map $J$ in the opposite direction, and then showing that there is a chain homotopy $H$, satisfying $i d-J I=D H+H D$, that induces an isomorphism on the level of cohomology. The chain map is constructed by means of moduli spaces $\mathcal{M}\left(O_{a}, O_{a^{\prime}}\right)$ of solutions of SeibergWitten equations on the cylinder $Y \times \mathbb{R}$ endowed with a metric $g_{t}+d t^{2}$ that varies between $g_{0}+d t^{2}$ and $g_{1}+d t^{2}$ along the cylinder. The main theorem, Theorem 6.1 is presented at the beginning of Section 6 , followed by a "model" proof of the easiest case of metrics and perturbation in the same chamber. The proof of the general statement of Theorem 6.1 will only be given in Section 6.3, after the necessary technical tools have been introduced.

The construction of the chain map, in the general case of Theorem 6.1, requires a careful analysis of the boundary structure of the moduli spaces 
$\mathcal{M}\left(O_{a}, O_{a^{\prime}}\right)$. In Section 6.1 we present the properties of the moduli spaces $\mathcal{M}\left(O_{a}, O_{a^{\prime}}\right)$. In Theorem 6.4, we prove the existence of a compactification for the moduli spaces $\mathcal{M}\left(O_{a}, O_{a^{\prime}}\right)$, obtained by adding fibered products of lower dimensional moduli spaces. This is the analogue of the results of Section 4.1 in the case of flow lines. We then give a transversality result, in Lemma 6.5 and Lemma 6.6, under the hypothesis that at least one of the critical orbits $O_{a}$ and $O_{a^{\prime}}$ has a free $U(1)$ action, or that the relative Morse index $\mu\left(\theta_{0}\right)-\mu\left(\theta_{1}\right)$ is non-negative. In the case of the moduli space $\mathcal{M}\left(\theta_{0}, \theta_{1}\right)$, with $\mu\left(\theta_{0}\right)-\mu\left(\theta_{1}\right)<0$, we show in Corollary 6.7 and in Lemma 6.8 that the transversality result fails. The moduli space $\mathcal{M}\left(\theta_{0}, \theta_{1}\right)$ consists in this case of a unique reducible solution and the linearization has a nontrivial cokernel of dimension equal to the index $\left|\mu\left(\theta_{0}\right)-\mu\left(\theta_{1}\right)\right|$. We prove in Theorem 6.9 the gluing theorem for all the non-singular boundary strata in $\mathcal{M}\left(O_{a}, O_{a^{\prime}}\right)^{*}$, namely those with trivial Cokernels. Similarly, we prove in Theorem 6.10 the gluing theorem for all the non-singular boundary strata in $\mathcal{M}^{P}\left(O_{a}, O_{b}\right)^{*}$.

Section 6.2 contains the general theory needed in order to deal with the gluing theorems in the presence of obstructions. It relies heavily on Taubes' technique developed in [60] and [59], together with Donaldson's results in [16]. We recall in Lemma 6.13 the necessary eigenvalue splitting for the Laplacians at the approximate solution, as proved in Lemma 4.12, Lemma 4.13, and Lemma 4.15 of Section 4.2. In Proposition 6.17 we introduce the obstruction bundle with the canonical obstruction section. In Lemma 6.14 we derive the fixed point argument which we use to complete the proof of the non-obstructed gluing of Theorem 4.9 and to formulate the gluing with obstruction in Proposition 6.17. In the remaining of Section 6.2 we analyze the modified boundary strata of $\mathcal{M}\left(O_{a}, O_{a^{\prime}}\right)^{*}$ and $\mathcal{M}^{P}\left(O_{a}, O_{b}\right)^{*}$. In Theorem 6.19 and Theorem 6.21 we identify the extra boundary components in $\mathcal{M}\left(O_{a}, O_{a^{\prime}}\right)^{*}$ due to the zeroes of the obstruction section, and we define in Proposition 6.20 the additional gluing maps. Similarly, in Theorem 6.22, we identify the extra boundary components in $\mathcal{M}^{P}\left(O_{a}, O_{b}\right)^{*}$ due to the zeroes of the obstruction sections, and we introduce the additional gluing maps.

In Section 6.3, we finally complete the proof of the topological invariance. The modification of the boundary structure discussed in Section 6.2 prescribes correction terms for the maps $I$ and $H$ (but not $J$ ), so that the identities $I D-D I=0$ and $i d-J I=D H+H D$ can still be satisfied. We first discuss, in Lemma 6.25 and Lemma 6.26, some more properties of the zeroes of the obstruction sections and some identities obtained by counting these zeroes, which are useful in checking the identities satisfied by the coefficients of the maps $I$ and $H$. We then introduce, in Definition 
6.27, the modified maps $I$ and $H$, and we prove, in Lemma 6.28, Lemma 6.29, Lemma 6.30, and Lemma 6.31, that the necessary identities hold, thus completing the proof of Theorem 6.1 on the topological invariance of the equivariant Seiberg-Witten Floer homology. It is clear, from the way the proof is structured, that the argument breaks down for the non-equivariant Floer homology. While the invariance within the same chamber is still verified (nothing changes in that part of the argument), in the case of metrics and perturbations in different chambers we need essentially the contribution of the reducible points in order to construct the chain map $I$ and chain homotopy $H$, as one can see form Definition 6.27, and the Lemmata 6.28, $6.29,6.30$, and 6.31 .

Section 7 deals with the wall crossing formula for the Casson-type invariant of homology spheres obtained as the Euler characteristic of the (nonequivariant) Seiberg-Witten Floer homology. In Section 7.1 we derive relations between the equivariant and the non-equivariant Floer homologies. We prove in Theorem 7.1 that, when we have $b^{1}(Y)>0$, the equivariant and the non-equivariant Floer homologies are isomorphic. This is not a surprising result: in fact, it is conceptually like considering the equivariant homology of a manifold with a free group action, for which it is well known that one recovers the homology of the quotient. In the case of rational homology spheres, we prove in Theorem 7.3 that there is an exact sequence relating the equivariant Floer homology with the non-equivariant and with the polynomial algebra $H_{*}(B U(1), \mathbb{R})$. The results of both Theorem 7.1 and 7.3 are derived by considering filtrations of the complexes and the associated spectral sequences, as in Lemma 7.2 and Lemma 7.4. We also give an explicit expression of the connecting homomorphism in the exact sequence in Proposition 7.5. The exact sequence that relates the metric independent equivariant Floer homology with the metric dependent non-equivariant one is the tool we exploit in Section 7.2, in order to derive, in a purely algebraic way, the wall crossing formula for the Euler characteristic in Theorem 7.7. The argument is based on the topological invariance of the equivariant Floer homology proved in Theorem 6.1, and the result of Proposition 7.6 on the counting of the ranks of the equivariant Floer groups. The result is generalized to multiple wall crossings, and to the case of the $J$-invariant perturbations of [14], in Propositions 7.8, 7.9, and in Corollary 7.10. In Section 7.3 we show how the same wall crossing formula can be derived geometrically by considering the local model of the parameterized moduli space of critical points $\mathcal{M}(g, \nu)$ along with a deformation of the metric and perturbation $(g, \nu)$.

Given the length of this work, we have thought it useful to add an index 
of notation at the end of the paper.

Acknowledgements. We are deeply grateful to T. Mrowka for the many invaluable comments and suggestions. We thank A. Carey and M. Rothenberg for the many useful conversations. We are grateful to L. Nicolaescu for useful remarks and suggestions. In the early stages of this work useful were comments of R. Brussee, G. Matić, R. Mazzeo, J.W. Morgan, and R.G. Wang. We thank the referee for the very detailed and useful comments and for many valuable suggestions on how to improve various sections of the manuscript. Parts of this work were carried out during visits of the two authors to the Max Planck Institut für Mathematik in Bonn, and visits of the first author to the University of Adelaide and to the Tata Institute of Fundamental Research. We thank these institutions for the kind hospitality and for support. The first author was partially supported by NSF grant DMS-9802480. The second author was supported by ARC fellowship.

\section{Non-equivariant Floer theory.}

There are two disjoint situations in which the non-equivariant SeibergWitten Floer homology can be defined. They have been considered in [13], [38], [64]. In this section, we first recall the general setting of SeibergWitten theory on 3-manifolds, and then we summarize the construction of non-equivariant Floer theory.

\subsection{Three dimensional Seiberg-Witten theory.}

Any three-manifold admits a Spin-structure. A choice of the metric determines a (non-canonical) choice of a "trivial" Spin-structure with spinor

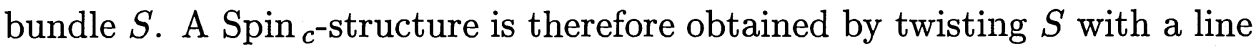
bundle $L$. Suppose given a three-manifold $Y$ with a $\operatorname{Spin}_{c}$ structure $S \otimes L$. Consider the space $\mathcal{A}_{k}$ of pairs $(A, \psi)$, where $A$ is a $U(1)$ connection on the line bundle $L$ and $\psi$ is a section of $S \otimes L$, endowed with a fixed $L_{k}^{2}$-Sobolev completion.

The group $\tilde{\mathcal{G}}_{k+1}$ is the gauge group of maps of $Y$ in $U(1)$ locally modeled on the Lie algebra

$$
\operatorname{Lie}\left(\tilde{\mathcal{G}}_{k+1}\right)=L_{k+1}^{2}\left(\Omega^{0}(Y, i \mathbb{R})\right),
$$

acting on pairs $(A, \psi)$ by

$$
\lambda:(A, \psi) \mapsto\left(A-2 \lambda^{-1} d \lambda, \lambda \psi\right) .
$$


The group $\mathcal{G}_{k+1}$ is the subgroup of $\tilde{\mathcal{G}}_{k+1}$ of gauge transformations $\lambda$ satisfying the condition

$$
\frac{i}{2 \pi} \int_{Y} c_{1}(L) \wedge \lambda^{-1} d \lambda=0 .
$$

Here $c_{1}(L)$ represents the Chern class of the line bundle $L$ that defines

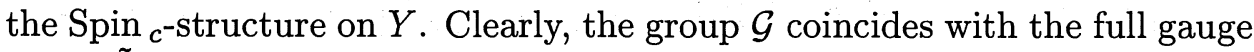
group $\tilde{\mathcal{G}}$ in the case when $c_{1}(L)=0$ rationally, hence in particular for rational homology spheres.

The functional

$$
\mathcal{C}(A, \psi)=\frac{-1}{2} \int_{Y}\left(A-A_{0}\right) \wedge\left(F_{A}+F_{A_{0}}\right)+\frac{1}{2} \int_{Y}\left\langle\psi, \partial_{A} \psi\right\rangle d v
$$

was first introduced by Kronheimer and Mrowka in the proof of the Thom conjecture [30]. It is defined on the space $\mathcal{A}$ of connections and sections and it is invariant under the action of the identity component $\mathcal{G}_{k+1}$. Thus, it descends to a real-valued functional on the space $\mathcal{B}_{k}=\mathcal{A}_{k} / \mathcal{G}_{k+1}$.

The first order increment of this functional defines a 1 -form on the $L_{k}^{2}$ tangent space $T \mathcal{A}$,

$$
\left.\mathcal{F}\right|_{(A, \psi, \rho)}(\alpha, \phi)=\int_{Y}-\alpha \wedge\left(F_{A}-* \sigma(\psi, \psi)\right)+\int_{Y}\left\langle\phi, \partial_{A} \psi\right\rangle .
$$

Thus, the gradient flow of the functional (3), with respect to the $L^{2}$-inner product, is given by the paths of connections and sections $(A(t), \psi(t))$ that satisfy the equations

$$
\frac{d}{d t} \psi=-\partial_{A} \psi
$$

and

$$
\frac{d}{d t} A=-* F_{A}+\sigma(\psi, \psi)
$$

where the 1-form $\sigma(\psi, \psi)$ is given in local coordinates by $\frac{1}{2}\left\langle e_{i} \psi, \psi\right\rangle e^{i}$.

These equations can be thought of as the three-dimensional reduction of the Seiberg-Witten equations on four-manifolds introduced in [66] (see also [4], [5], [13], [38], [63], [64]). In fact, we can consider the four-manifold $Y \times \mathbb{R}$ with a cylindrical metric $g+d t^{2}$, and with $\operatorname{Spin}_{c}$ structure determined by the pullback of $S \otimes L$ via the projection $\pi: Y \times \mathbb{R} \rightarrow Y$. Thus we have $S^{+} \otimes L \cong \pi^{*}(S \otimes L)$. Over $Y$ we have $S^{+} \otimes L \cong S^{-} \otimes L$ under Clifford 
multiplication by $d t$, and the identifications $\Omega^{2+}(Y \times \mathbb{R}, i \mathbb{R}) \cong \pi^{*}\left(\Omega^{1}(Y, i \mathbb{R})\right)$ and $\Omega^{1}(Y \times \mathbb{R}, i \mathbb{R}) \cong \pi^{*}\left(\Omega^{0}(Y, i \mathbb{R}) \oplus \Omega^{1}(Y, i \mathbb{R})\right)$, that is $\pi^{*}(\rho(t))=* \rho(t)+$ $\rho(t) \wedge d t$ and $\pi^{*}(f(t), \alpha(t))=\alpha(t)+f(t) d t$ on $Y \times \mathbb{R}$.

Consider a pair $(\mathbb{A}, \Psi)$ on $Y \times \mathbb{R}$, where $\mathbb{A}$ is a $U(1)$ connection on the determinant line bundle of $S^{+} \otimes L$ and $\Psi$ is a section of the spinor bundle $S^{+} \otimes L$. An element $(\mathbb{A}, \Psi)$ is in a temporal gauge if the $d t$-component of $\mathbb{A}$ vanishes identically. Thus, a path $(A(t), \psi(t))$ corresponds to an element $(\mathbb{A}, \Psi)$ in a temporal gauge.

Lemma 2.1. The Seiberg-Witten equations (5) and (6) on $Y \times \mathbb{R}$ are equivalent to

$$
F_{\AA}^{+}=\Psi \cdot \bar{\Psi}
$$

and

$$
D_{\mathbb{A}} \Psi=0,
$$

where $\Psi \cdot \bar{\Psi}$ is the self-dual two-form given in local coordinates by $\Psi \cdot \bar{\Psi}=$ $\left\langle e_{i} e_{j} \Psi, \Psi\right\rangle e^{i} \wedge e^{j}$ and $D_{\mathbb{A}}$ is the Dirac operator twisted with the connection $\mathbb{A}$ acting on sections of $S^{+} \otimes L$.

Proof. The Dirac operator $D_{\mathbb{A}}: S^{+} \otimes L \rightarrow S^{-} \otimes L$ on the four-manifold, twisted with the connection $\mathbb{A}$, has the form

$$
D_{\mathbb{A}}=\partial_{t}+\partial_{A(t)},
$$

where $\partial_{A}$ is the self-adjoint Dirac operator on $Y$ twisted with the time dependent connection $A(t)$. For the curvature equation (7), write $F_{\mathbb{A}}^{+}=$ $\frac{1}{2}\left(F_{\AA}+*_{4} F_{\AA}\right)$. Since $F_{\AA}^{-}$acts trivially on the positive spinors, the action of $F_{\mathbb{A}}^{+}$corresponds precisely to the action of $\frac{d A}{d t}+*_{3} F_{A}$. Here we have introduced the notation $*_{4}$ and $*_{3}$ to distinguish the Hodge $*$-operator on the 4-manifold $Y \times \mathbb{R}$ and on the 3-manifold $Y$. In the following, we shall drop the subscript, since it will be clear which $*$-operator is being used. Thus, given the expression of the 2-form $\Psi \cdot \bar{\Psi}$ and of the one form $\sigma(\psi(t), \psi(t))$, we can write equation (7) as

$$
\frac{1}{2}\left(F_{i t}+\epsilon^{i t j k} F_{j k}\right) e^{i} \wedge d t=\frac{1}{4}\left\langle e_{i} e_{t} \psi, \psi\right\rangle e^{i} \wedge d t,
$$

with an implicit sum over repeated indices and the symbol $\epsilon$ denoting the sign of the permutation $\{i t j k\}$. Upon applying the $*_{3}$ operator and using the 
identification of $S^{+} \otimes L$ and $S^{-} \otimes L$ on $Y \times \mathbb{R}$ under Clifford multiplication by $d t$, we can identify this equation with the equation (6).

The Seiberg-Witten equations on the 4-manifold $Y \times \mathbb{R}$ are preserved by the action of the gauge group $\tilde{\mathcal{G}}_{Y \times \mathbb{R}}$ of maps of $Y \times \mathbb{R}$ in $U(1)$. Any element $(\mathbb{A}, \Psi)$ can be transformed into a temporal gauge by effect of a gauge transformation. Suitable Sobolev completions of these spaces will be introduced in Proposition 2.9. For a general overview of the Seiberg-Witten theory on four-manifold see [15], [39], [45], [56].

The critical points of the functional $\mathcal{C}$ are pairs $(A, \psi)$ that satisfy

$$
\begin{gathered}
\partial_{A} \psi=0, \\
* F_{A}=\sigma(\psi, \psi) .
\end{gathered}
$$

Let $\mathcal{M}_{k}$ be the moduli space of solutions of (9) in $\mathcal{B}_{k}$ and $\tilde{\mathcal{M}}_{k}$ be the moduli space of solutions in $\tilde{\mathcal{B}}_{k}=\mathcal{A}_{k} / \tilde{\mathcal{G}}_{k+1}$.

Lemma 2.2. By elliptic regularity, $\mathcal{M}_{k}$ can be represented by smooth elements.

Lemma 2.2 follows from the Sobolev embedding theorems. In the following we drop the subscript $k$ and just write $\mathcal{M}$ for the moduli space of critical points.

The deformation complex that determines the virtual dimension of $\mathcal{M}$ is given by

$$
\begin{aligned}
0 \rightarrow \Omega^{0}(Y, i \mathbb{R}) \oplus \Omega^{1}(Y, i \mathbb{R}) \oplus & \Gamma(S \otimes L) \\
& \stackrel{L}{\rightarrow} \Omega^{0}(Y, i \mathbb{R}) \oplus \Omega^{1}(Y, i \mathbb{R}) \oplus \Gamma(S \otimes L) \rightarrow 0,
\end{aligned}
$$

with an index zero Fredholm operator

$$
\left.L\right|_{(A, \psi)}(f, \alpha, \phi)=\left\{\begin{array}{l}
\left.T\right|_{(A, \psi)}(\alpha, \phi)+\left.G\right|_{(A, \psi)}(f) \\
\left.G^{*}\right|_{(A, \psi)}(\alpha, \phi)
\end{array}\right.
$$

defined between the $L_{k}^{2}$ and the $L_{k-1}^{2}$-Sobolev completions of the spaces above, where the operator

$$
\left.G\right|_{(A, \psi)}(f)=(-d f, f \psi)
$$

is the infinitesimal action of the gauge group and $G^{*}$ is the adjoint with respect to the $L^{2}$ pairing. The map $T$ is the linearization of the equation 
(9) at a pair $(A, \psi)$,

$$
\left.T\right|_{(A, \psi)}(\alpha, \phi)=\left\{\begin{array}{l}
* d \alpha-2 i \operatorname{Im} \sigma(\psi, \phi) \\
\partial_{A} \phi+\alpha \psi
\end{array}\right.
$$

The Hessian of the functional $\mathcal{C}$ is given by a quadratic form in the increment $(\alpha, \phi) \in T \mathcal{A}$,

$$
\left.\nabla \mathcal{F}\right|_{(A, \psi)}(\alpha, \phi)=\langle\alpha, * d \alpha-2 i \operatorname{Im} \sigma(\psi, \phi)\rangle+\left\langle\phi, \partial_{A} \phi+\alpha \psi\right\rangle .
$$

This descends to the same operator $T$ on the $L_{k}^{2}$-tangent space $T_{[A, \psi]} \mathcal{B}_{k}$, when $(A, \psi)$ is a solution of (9), since the condition

$$
G_{(A, \psi)}^{*}\left(T_{(A, \psi)}(\alpha, \phi)\right)=0
$$

is satisfied. The operator $T$ is essentially self-adjoint.

As in the case of Donaldson theory [1], the linearization is a first order elliptic operator, hence its spectrum is not in general bounded from below, and this affects the definition of the index of critical points, as we are going to discuss in Proposition 2.12.

We consider the functional $\mathcal{C}$ perturbed with a co-closed 1-form $\rho$,

$$
\mathcal{C}_{\rho}(A, \psi)=\mathcal{C}(A, \psi)-2 i \int_{Y}\left(A-A_{0}\right) \wedge * \rho .
$$

We have the corresponding perturbed critical point equations

$$
\begin{gathered}
\partial_{A} \psi=0, \\
* F_{A}=\sigma(\psi, \psi)+2 i \rho .
\end{gathered}
$$

Notice that the first integral cohomology group of $Y$ counts the homotopy classes of gauge transformations, namely $H^{1}(Y, \mathbb{Z}) \cong \pi_{0}(\tilde{\mathcal{G}})$ under the identification

$$
\lambda \rightarrow h=\left[\frac{i}{2 \pi} \lambda^{-1} d \lambda\right] \in H^{1}(Y, \mathbb{Z}) .
$$

We denote with $H$ the subgroup of the classes $h \in H^{1}(Y, \mathbb{Z})$ that satisfy

$$
\left\langle c_{1}(L) \cup h,[Y]\right\rangle=\frac{i}{2 \pi} \int_{Y} c_{1}(L) \wedge \lambda^{-1} d \lambda=0 .
$$

We have the following compactness result for the set of critical points of the Seiberg-Witten functional. 
Proposition 2.3. The moduli space $\tilde{\mathcal{M}}_{\rho}$ of solutions of (15) modulo the action of the full gauge group $\tilde{\mathcal{G}}$ is compact. The space $\mathcal{M}_{\rho}$ of solutions, modulo the subgroup $\mathcal{G} \subset \tilde{\mathcal{G}}$ of gauge transformations satisfying (2), consists of a copy of $\tilde{\mathcal{M}}_{\rho}$ for each class in $H^{1}(Y, \mathbb{Z}) / H$. $\mathcal{M}_{\rho}$ is compact iff $H^{1}(Y, \mathbb{Z}) / H$ is finite. In particular, $\mathcal{M}_{\rho}$ is compact if $b^{1}(Y)=0$.

Notice that the condition that $H^{1}(Y, \mathbb{Z}) / H$ is finite corresponds to $c_{1}(L)=0$ rationally.

Proposition 2.4. If the manifold $Y$ has $b^{1}(Y)>0$, then, for an open set of small perturbations, the perturbed equations (15) do not admit reducible solutions. Moreover, by the Sard-Smale theorem, for a generic choice of $\rho$ the corresponding moduli space $\tilde{\mathcal{M}}_{\rho}$ is a smooth manifold that is cut out transversely by the equations. $\tilde{\mathcal{M}}_{\rho}$ is compact and zero-dimensional, hence it consists of a finite set of points.

All the moduli spaces come with a natural orientation defined by the determinant line bundle of the Fredholm linearization, as in [17].

When $Y$ is a homology sphere the perturbation $\rho$ can be written as $\rho=* d \nu$ and the equations (15) admit one gauge class of reducible solutions $[\nu, 0]$.

Proposition 2.5. If $Y$ is a homology sphere, then there is a unique gauge class of reducible solutions $\theta=[\nu, 0]$ of (15) with $\rho=* d \nu$. If the metric on $Y$ is such that $\operatorname{Ker}\left(\partial_{\nu}^{g}\right)=0$, where $\partial_{\nu}^{g}$ is the self-adjoint Dirac operator on $Y$ twisted with the $U(1)$-connection $\nu$, then the analysis of the local Kuranishi model shows that the reducible point is isolated and non-degenerate.

Propositions 2.3, 2.4, and 2.5 are proven in [64], [38], and [13] respectively. See also the more recent [35].

\subsection{Chamber structure.}

In this subsection we prove that $\operatorname{Ker}\left(\partial_{\nu}^{g}\right)=0$ is satisfied for generic metrics. The condition $\operatorname{Ker}\left(\partial_{\nu}^{g}\right) \neq 0$ defines a chamber structure in the space of metrics. The walls form a stratified space with a codimension one top stratum and higher codimensional strata, in the sense described in Theorem 2.8.

Notice that there is a more abstract approach [28] describing a stratification of the space of Fredholm operators by their index and kernel dimension. However, the result we are interested in does not follow directly by simply applying the results of [28]. In fact, we know from the general result of 
Section 1 of [28] that the space $\mathcal{F}_{0}(S, Y)$ of index zero Fredholm operators, acting on the bundle $S$ (or $S \otimes L$ ) over $Y$, has the structure of a real analytic manifold, with a stratification given by the sets $\mathcal{F}_{0, n}(S, Y)$ of Fredholm operators of index zero with the dimension of the kernel equal to $n$. In our specific problem, however, we are considering the particular map from the space $\mathcal{M} e t \times Z^{1}(Y, \mathbb{R})$ of metrics and perturbations $(g, * d \nu)$ to the space $\mathcal{F}_{0}(S, Y)$ given by

$$
\begin{gathered}
\mathcal{X}: \mathcal{M} e t \times Z^{1}(Y, \mathbb{R}) \rightarrow \mathcal{F}_{0}(S, Y) \\
(g, * d \nu) \mapsto \partial_{\nu}^{g}=: \mathcal{X}(g, \nu) .
\end{gathered}
$$

Thus, we need a specific result that shows how the image of the map $\mathcal{X}$ lies in $\mathcal{F}_{0}(S, Y)$ with respect to the stratification of [28]. This is the purpose of our Theorem 2.7 and Theorem 2.8. Although it is quite possible that a "proof by library search" of these results may be provided combining the circle of ideas in [28], [37], and the more recent [21], we prefer to give a direct proof that covers our specific case. A note should be added: during the long period between the initial submission of this paper and the completion of its publishing process, a discussion of the chamber structure has also appeared in [35] and [55].

Remark 2.6. Suppose $Y$ is a homology sphere. There is a quaternion structure on the spinor bundle $S, J: S \rightarrow \bar{S}$ locally given by $\left(z_{1}, z_{2}\right) \mapsto\left(-\bar{z}_{2}, \bar{z}_{1}\right)$. The action of $J$ extends to the configuration space $\mathcal{A}$ as $(A, \psi) \mapsto\left(A^{*}, J \psi\right)$. This means that $J$ acts on $\nu$ by $J \nu=-\nu$, hence

$$
J\left(\partial_{\nu}^{g} \psi\right)=\partial_{-\nu}^{g} J \psi
$$

In other words, the Dirac operator $\partial^{g}$ on a three-manifold $Y$ is quaternion linear, but the twisted Dirac operator $\partial_{\nu}^{g}$ is only complex linear.

A $J$-invariant perturbation of the Seiberg-Witten equations has been constructed by W. Chen [14], where the Dirac operator is perturbed with a smooth real function $f$ on $Y$. This choice of perturbation leads to a different chamber structure in the space of metrics which lies inside one chamber of the more general perturbation

$$
\begin{gathered}
\partial_{A} \psi=f \psi, \\
* F_{A}=\sigma(\psi, \psi)+* d \nu .
\end{gathered}
$$

In this setting, transversality can be achieved by adding a perturbation by a function of the holonomy. This choice of perturbations lead to a different 
wall crossing formula. However, we are going to show in Section 7, Proposition 7.9 and Corollary 7.10, that the wall crossing formula for the $J$-invariant perturbations can be derived with the same technique that we employ in the case on perturbations $\nu$.

The following theorems discuss the chamber structure in the space of metrics and perturbations.

Theorem 2.7. Let Met be the space of all Riemannian metrics on a homology 3-sphere $Y$. Consider the twisted Dirac operator $\partial_{\nu}^{g}$ associated with the chosen metric $g$ and the connection $\nu$. The condition $\operatorname{Ker} \partial_{\nu}^{g} \neq 0$ determines a real codimension one subset in the space of $M e t \times Z^{1}(Y, i \mathbb{R})$.

Proof. Let $g_{0}$ be a metric on $Y$ such that $\operatorname{Ker} \partial_{\nu_{0}}^{g_{0}} \neq 0$ for the connection $\nu_{0}$. We can decompose the spinor space as $\mathcal{H} \oplus \mathcal{H}^{\perp}$, under the $L^{2}$ inner product, where $\mathcal{H}=\operatorname{Ker} \partial_{\nu_{0}}^{g_{0}}$, equipped with a Hermitian metric from the

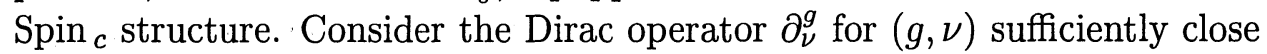
to $\left(g_{0}, \nu_{0}\right)$ in the $C^{\infty}$-topology. Under the isometry identification of the spinor spaces for $g_{0}$ and $g$, the Dirac operator for $g$ can be considered to act on the spinor space of $g_{0}$, still denoted by $\partial_{\nu}^{g}$.

Claim 1. $\partial_{\nu}^{g}$ acting on the spinor space $S_{g_{0}}$ is self-adjoint if and only if the metrics $g_{0}$ and $g$ define the same volume element.

Suppose $d v o l_{g}=f d v o l_{g_{0}}$ for a positive function $f$ on $Y$, then with a direct calculation we get

$$
\left\langle\partial_{\nu}^{g} \psi, \phi\right\rangle_{g_{0}}=\left\langle\psi, f \partial_{\nu}^{g}\left(f^{-1} \phi\right)\right\rangle_{g_{0}} .
$$

Claim 1 is then immediate. Denote by $M e t^{0}$ the space of metrics which have the same volume element as the metric $g_{0}$.

Claim 2. If two metrics $g_{1}$ and $g_{2}$ are conformal, that is, $g_{1}=e^{2 u} g_{2}$ for a real function on $Y$, then the multiplication by $e^{-u}$ defines an isomorphism between $\operatorname{Ker} \partial_{\nu}^{g_{1}}$ and $\operatorname{Ker} \partial_{\nu}^{g_{2}}$.

This is the consequence of the following relation: under the isometry identification of the spinor spaces for $g_{1}$ and $g_{2}$, we have (see [26] or [33] Theorem II.5.24)

$$
\partial_{\nu}^{g_{1}}=e^{-u} \partial_{\nu}^{g_{2}} e^{u}
$$

Notice that, in the formula for the variation of the Dirac operator under conformal changes in the metric, different conventions are used in the literature. Here we are following the convention and notation of [26]. 
Therefore, we only need to prove that the condition $\operatorname{Ker} \partial_{\nu}^{g} \neq 0$ determines a real codimension one subset in the space of $M e t^{0} \times Z^{1}(Y, i \mathbb{R})$.

We want to reduce the problem of the existence of solutions of the equation $\partial_{\nu}^{g} \psi=0$ to a finite dimensional problem on $\mathcal{H}$.

As a map from $M e t^{0} \times Z^{1}(Y, i \mathbb{R}) \times \Gamma(S)$ to $\Gamma(S)$, the linearization of the equation $\partial_{\nu}^{g} \psi=0$ at $\left(g_{0}, \nu_{0}, 0\right)$ is invertible when restricted to $\mathcal{H}^{\perp}$. Thus, the implicit function theorem provides a unique map $q: \mathcal{U} \rightarrow \mathcal{H}^{\perp}$ defined on a neighborhood $\mathcal{U}$ of $\left(g_{0}, \nu_{0}, 0\right)$ in $M e t^{0} \times Z^{1}(Y, i \mathbb{R}) \times \mathcal{H}$, such that

$$
(1-\Pi) \partial_{\nu}^{g}(\phi+q(g, \nu, \phi))=0
$$

for all $(g, \nu, \phi) \in \mathcal{U}$, where $\Pi$ is the projection onto $\mathcal{H}$.

Therefore, the operator $\partial_{\nu}^{g}$ has non-trivial kernel if and only if the equation

$$
\Pi \partial_{\nu}^{g}(\phi+q(g, \nu, \phi))=0
$$

admits solutions in $\mathcal{H}$. This is a finite dimensional problem. Define a map

$$
L:\left(M e t^{0} \times Z^{1}(Y, i \mathbb{R})\right) \cap \mathcal{U} \longrightarrow U(\mathcal{H})
$$

by setting

$$
L(g, \nu)(\phi)=\Pi \partial_{\nu}^{g}(\phi+q(g, \nu, \phi)) .
$$

Direct calculation implies that $L(g, \nu)$ is a Hermitian transformation of the space $\mathcal{H}$, that $L(g, \nu) \in U(\mathcal{H})$, where $U(\mathcal{H})$ is the Hermitian transformation group on $\mathcal{H}$. The kernel of $\partial_{\nu}^{g}$ is non-trivial if and only if the kernel of $L(g, \nu)$ is non-trivial. The determinant is a real-valued function on $U(\mathcal{H})$. Thus, we have a real-valued function $f(g, \nu)=\operatorname{det}(L(g, \nu))$ on the neighborhood of $\left(g_{0}, \nu_{0}\right)$ in $M e t^{0} \times Z^{1}(Y, i \mathbb{R})$. Those $(g, \nu)$ with non-trivial kernel have value 0 for this function.

Now we only need to check that the derivative of $f(g, \nu)$ at $\left(g_{0}, \nu_{0}\right)$ is surjective, then the condition $\operatorname{Ker} \partial_{\nu}^{g} \neq 0$ determines a real codimension one subset in the space of $M e t^{0} \times Z^{1}(Y, i \mathbb{R})$ by Morse theory. It can be checked by differentiating $f(g, \nu)$ at $\left(g_{0}, \nu_{0}\right)$ along $(0, \alpha)$-direction, for $\alpha \in \Omega^{1}(Y, i \mathbb{R})$. Since

$$
D f_{\left(g_{0}, \nu_{0}\right)}(0, \alpha)=\operatorname{Tr}\left(\phi \mapsto \Pi\left(\frac{1}{2} \alpha . \phi\right)\right),
$$

which is non-zero for suitable choice of $\alpha$.

Theorem 2.7 now follows from Claim 2 and the fact that any metric is conformally equivalent to a metric in $M e t^{0}$. 
Let $\mathcal{W}$ denote the wall in the space of metrics and perturbations $M e t \times$ $Z^{1}(Y, i \mathbb{R})$

$$
\mathcal{W}=\left\{(g, * d \nu) \mid \operatorname{Ker} \partial_{\nu}^{g} \neq 0\right\}
$$

Motivated by Chen's work [14], we analyze more carefully the structure of $\mathcal{W}$. This analysis will be useful in Section 7, in establishing the results on the wall crossing formulae.

Theorem 2.8. $\mathcal{W}$ is a stratified space with the top stratum $\mathcal{W}_{1}$ consisting of those $(g, \nu)$ with $\operatorname{Ker} \partial_{\nu}^{g} \cong \mathbb{C}$. In general, the set of pairs $(g, \nu)$ with $\operatorname{Ker} \partial_{\nu}^{g} \cong \mathbb{C}^{n}$ is a codimension $2 n-1$ subset $\mathcal{W}_{n}$ in $M$ et $\times Z^{1}(Y, i \mathbb{R})$.

Proof. As in the proof of Theorem 2.7, we only need to prove the result for $(g, \nu)$ in $M e t^{0} \times Z^{1}(Y, i \mathbb{R})$ (see Claim 1 and Claim 2 in the proof of Theorem 2.7). Consider a real Hilbert bundle $\mathcal{L}$ over

$$
M e t^{0} \times Z^{1}(Y, i \mathbb{R}) \times\left(L_{1}^{2}(S)-\{0\}\right)
$$

whose fiber over $(g, \nu, \psi)$ is

$$
\mathcal{L}_{(g, \nu, \psi)}=\left\{\phi \in L_{1}^{2}(S) \mid \operatorname{Re}\langle\phi, i \psi\rangle_{g}=0\right\}
$$

Define a section $\zeta$ of $\mathcal{L}$ by assigning to $(g, \nu, \psi)$ the element $\partial_{\nu}^{g} \psi$.

Claim. $\zeta$ is transverse to the zero section of $\mathcal{L}$.

We need to prove that the differential map of $\zeta$ is surjective at zeroes of $\zeta$. Suppose that $\left(g_{0}, \nu_{0}, \psi_{0}\right)$ (with $\psi \neq 0$ ) satisfies $\partial_{\nu_{0}}^{g_{0}} \psi_{0}=0$. Differentiating $\zeta$ with respect to the directions tangent to $Z^{1}(Y, i \mathbb{R}) \times\left(L_{1}^{2}(S)-\{0\}\right)$ only, we see that the differential map is

$$
\begin{aligned}
\mathcal{D} \zeta: \quad \Omega_{L_{2}^{2}}^{1}(Y, i \mathbb{R}) \oplus L_{1}^{2}(S) & \longrightarrow\left\{\phi \in L_{1}^{2}(S) \mid \operatorname{Re}\langle\phi, i \psi\rangle_{g}=0\right\} \\
\left(\nu_{1}, \psi_{1}\right) & \mapsto \partial_{\nu_{0}}^{g_{0}} \psi_{1}+\frac{1}{2} \nu_{1} . \psi_{0} .
\end{aligned}
$$

If $\phi \in \mathcal{L}_{\left(g_{0}, \nu_{0}, \psi_{0}\right)}$ is orthogonal to the image of $\mathcal{D} \zeta$, then $\phi$ satisfies:

$$
\left\{\begin{array}{l}
(1) \cdot \operatorname{Re}\left\langle\phi, i \psi_{0}\right\rangle_{g_{0}}=0, \\
\text { (2). } \operatorname{Re}\left\langle\phi, \nu_{1} \cdot \psi_{0}\right\rangle_{g_{0}}=0, \quad \text { for any } \nu_{1} \in \Omega^{1}(Y, i \mathbb{R}) . \\
\text { (3). } \partial_{\nu_{0}}^{g_{0}} \phi=0 .
\end{array}\right.
$$


From the second equation we see that there exists a function $f: Y \rightarrow \mathbb{R}$ such that $\phi=i f \psi_{0}$. Substitute this into equation (3), using $\partial_{\nu_{0}}^{g_{0}} \psi_{0}=0$. We obtain $d f=0$ which implies $f=C$ is a constant function. Then

$$
\operatorname{Re}\left\langle i C \psi_{0}, i \psi_{0}\right\rangle_{g_{0}}=C\left|\psi_{0}\right|^{2}
$$

implies $C=0$. Therefore, $\phi=0$, that means, $\mathcal{D} \zeta$ is surjective at $\left(g_{0}, \nu_{0}, \psi_{0}\right)$. It is easy to see that the index of $\mathcal{D} \zeta$ is the index of $\partial_{\nu_{0}}^{g_{0}}$, which is 1 , since $i \psi_{0}$ is orthogonal to the image of $\partial_{\nu_{0}}^{g_{0}}$.

From the Claim, $\zeta^{-1}(0)$ is a Banach manifold and the projection

$$
\Pi: \zeta^{-1}(0) \rightarrow M e t^{0} \times \Omega^{1}(Y, i \mathbb{R})
$$

is a Fredholm operator of index 1. Note that for any $(g, \nu) \in M e t^{0} \times$ $\Omega^{1}(Y, i \mathbb{R})$ we have $\Pi^{-1}(g, \nu)=\operatorname{Ker} \partial_{\nu}^{g}$.

Moreover, at $\left(g_{0}, \nu_{0}, \psi_{0}\right)$ we have

$$
\left\{\begin{array}{l}
\operatorname{Ker}\left(\Pi_{*}\right)=\left\{\phi \mid \partial_{\nu_{0}}^{g_{0}} \phi=0\right\} \\
\operatorname{dim} \operatorname{Ker}\left(\Pi_{*}\right)-\operatorname{dim} \operatorname{Coker}\left(\Pi_{*}\right)=1 .
\end{array}\right.
$$

Therefore, $\operatorname{dim}$ Coker $\left(\Pi_{*}\right)=\operatorname{dim} \operatorname{Ker}\left(\Pi_{*}\right)-1$. Then the Theorem follows, with the top stratum of codimension one described in Theorem 2.7, and the stratification given by

$$
\mathcal{W}_{n}=\mathcal{X}^{-1}\left(\mathcal{F}_{0,2 n}(S, Y) \cap \mathcal{X}\left(\mathcal{M} e t \times Z^{1}(Y, \mathbb{R})\right)\right),
$$

where $\mathcal{X}$ is the map of (16). Thus, the structure of stratified set on $\mathcal{W}$ is induced by the structure on Image $(\mathcal{X})$ inside the stratified set $\mathcal{F}_{0}(S, Y)$.

Notice that on any three-manifold it is possible to find special metrics for which the dimension of the space of harmonic spinors is arbitrarily large. The result for $S^{3}$ was proved by Hitchin [26] and recently generalized to all manifolds of dimension 3 mod 4 by Bär [9].

\subsection{Perturbation of flow lines.}

We introduce suitable moduli spaces of gradient flow lines connecting critical points. We prove in the Section 2.3, Proposition 2.14 that, generically, these are smooth manifolds that are cut out transversely, hence with the dimension prescribed by the index theorem. This property depends on an accurate choice of a class of perturbations for the gradient flow equations. 
Consider the space of connections and spinor sections $(\mathbb{A}, \Psi)$ on $Y \times \mathbb{R}$ with the product metric $g+d t^{2}$, topologized with the weighted Sobolev norms [24], [36]. Here we choose the weight $e_{\delta}(t)=e^{\tilde{\delta} t}$, where $\tilde{\delta}$ is a smooth function with bounded derivatives, $\tilde{\delta}: \mathbb{R} \rightarrow[-\delta, \delta]$ for some fixed positive number $\delta$, such that $\tilde{\delta}(t) \equiv-\delta$ for $t \leq-1$ and $\tilde{\delta}(t) \equiv \delta$ for $t \geq 1$. The $L_{k, \delta}^{2}$ norm is defined as $\|f\|_{2, k, \delta}=\left\|e_{\delta} f\right\|_{2, k}$, where the Sobolev norms are defined with respect to a fixed reference connection $\mathbb{A}_{0}$ in $L_{k, l o c}^{2}$. By the Sobolev multiplication theorem of Proposition 2.9 below, the spaces $L_{k, \delta, \mathbb{A}_{0}}^{2}$ and $L_{k, \delta, \mathbb{A}_{1}}^{2}$ are equal whenever we have $\mathbb{A}_{0}-\mathbb{A}_{1}=\alpha_{0}+\alpha_{1}$, with $\alpha_{0}$ in $L_{\ell, \delta, \mathbb{A}_{0}}^{2}$ and $\alpha_{1}$ is $\mathcal{C}^{\ell}$ bounded, with $\ell \geq k-1$, cf. [46]. The weight $e_{\delta}$ imposes an exponential decay as asymptotic condition along the cylinder. A proof of the following Proposition can be found, for instance, in Section 9 of [20].

Proposition 2.9. Let $Y$ be a compact oriented three-manifold endowed with a fixed Riemannian metric $g_{0}$. Consider the cylinder $Y \times \mathbb{R}$ with the metric $g_{0}+d t^{2}$. The weighted Sobolev spaces $L_{k, \delta}^{2}$ on the manifold $Y \times \mathbb{R}$ satisfy the following Sobolev embeddings.

(i) The embedding $L_{k, \delta}^{2} \hookrightarrow L_{k-1, \delta}^{2}$ is compact for all $k \geq 1$.

(ii) If $k>m+2$ we have a continuous embedding $L_{k, \delta}^{2} \hookrightarrow \mathcal{C}^{m}$.

(iii) If $k>m+3$ the embedding $L_{k, \delta}^{2} \hookrightarrow \mathcal{C}^{m}$ is compact.

(iv) If $2<k^{\prime}$ and $k \leq k^{\prime}$ the multiplication map $L_{k, \delta}^{2} \otimes L_{k, \delta}^{2} \stackrel{m}{\rightarrow} L_{k, 2 \delta}^{2}$ is continuous.

Consider a metric $g_{t}+d t^{2}$ on the cylinder $Y \times \mathbb{R}$ such that for a fixed $T$ we have $g_{t} \equiv g_{0}$ for $t \geq T$ and $g_{t} \equiv g_{1}$ for $t \leq-T$ and $g_{t}$ varies smoothly when $t \in[-1,1]$. The same Sobolev embedding theorems hold for the $L_{k, \delta}^{2}$ spaces on $\left(Y \times \mathbb{R}, g_{t}+d t^{2}\right)$.

Choose smooth representatives $\left(A_{0}, \psi_{0}\right)$ and $\left(A_{1}, \psi_{1}\right)$ of $a$ and $b$ in $\mathcal{M}$. Choose a smooth path $(A(t), \psi(t))$ such that for $t \leq 0$ it satisfies $(A(t), \psi(t)) \equiv\left(A_{0}, \psi_{0}\right)$ and for $t \geq 1$ it is $(A(t), \psi(t)) \equiv\left(A_{1}, \psi_{1}\right)$. The configuration space $\mathcal{A}_{k, \delta}(a, b)$ is given by the space of pairs $(\mathbb{A}, \Psi)$ on $Y \times \mathbb{R}$ satisfying

$$
(\mathbb{A}, \Psi) \in(A(t), \psi(t))+L_{k, \delta}^{2}\left(\Omega^{1}(Y \times \mathbb{R}) \oplus \Gamma\left(S^{+} \otimes L\right)\right) .
$$

Consider the group $\mathcal{G}_{k+1, \delta}(a, b)$ of gauge transformations in $\tilde{\mathcal{G}}_{Y \times \mathbb{R}}$, locally modeled on $L_{k+1, \delta}^{2}\left(\Omega^{0}(Y \times \mathbb{R}, i \mathbb{R})\right)$, that approach elements $\lambda_{ \pm \infty}$ in 
the stabilizers $G_{a}$ and $G_{b}$ of $\left(A_{0}, \psi_{0}\right)$ and $\left(A_{1}, \psi_{1}\right)$ as $t \rightarrow \pm \infty$. This gauge group acts on $\mathcal{A}_{k, \delta}(a, b)$ and we can form the quotient $\mathcal{B}_{k, \delta}(a, b)$. There is an action of $\mathbb{R}$ by translations on $\mathcal{B}_{k, \delta}(a, b)$.

We consider the perturbed gradient flow equations for a path $(A(t), \psi(t))$,

$$
\frac{d}{d t} \psi(t)=-\partial_{A(t)} \psi(t)
$$

and

$$
\frac{d}{d t} A(t)=\sigma(\psi(t), \psi(t))-* F_{A(t)}+2 i \rho+2 q_{(\mathbb{A}, \Psi)}(t) .
$$

Equations (18) and (19) can be rewritten in terms of pairs $(\mathbb{A}, \Psi)$ in the form

$$
D_{\mathbb{A}} \Psi=0
$$

and

$$
F_{\mathbb{A}}^{+}=\Psi \cdot \bar{\Psi}+i \mu+P_{(\mathbb{A}, \Psi)},
$$

as proved in Lemma 2.1 .

The perturbation $P=* q+q \wedge d t$ is a function of $\mathcal{B}(a, b)$ to $\pi^{*}\left(\Omega^{1}(Y, i \mathbb{R})\right) \cong \Omega^{2+}(Y \times \mathbb{R}, i \mathbb{R})$, such that the corresponding equations in a temporal gauge (18) and (19) are preserved under the action of $\mathbb{R}$ by reparameterization of the path $(A(t), \psi(t))$. The class of such perturbations is described as follows.

Definition 2.10. The space of perturbations $\mathcal{P}$ is the space of maps

$$
P: \mathcal{B}_{k, \delta}(a, b) \rightarrow L_{k, \delta}^{2} \Omega^{2+}(Y \times \mathbb{R}, i \mathbb{R}),
$$

that satisfy the following conditions.

(1) $P_{(\mathbb{A}, \Psi)}=* q_{(\mathbb{A}, \Psi)}(t)+q_{(\mathbb{A}, \Psi)}(t) \wedge d t$, where $q_{(\mathbb{A}, \Psi)}(t)$ satisfies

$$
q_{(\mathbb{A}, \Psi)^{T}}(t)=q_{(\mathbb{A}, \Psi)}(t+T),
$$

for any $T \in \mathbb{R}$, where $(\mathbb{A}, \Psi)^{T}$ is the $T$-translate of $(\mathbb{A}, \Psi)$.

(2) The $L_{k, \delta}^{2}$-norm of the perturbation $P_{(\mathbb{A}, \Psi)}$ is bounded uniformly with respect to $(\mathbb{A}, \Psi)$. 
(3) The linearization $\mathcal{D} P_{(\mathbb{A}, \Psi)}$ is a pseudodifferential operator of order $<1$. In particular $\mathcal{D} P_{(\mathbb{A}, \Psi)}$ is a compact operator from the $L_{k, \delta}^{2}$ to the $L_{k-1, \delta}^{2}$ tangent spaces.

(4) For all $l \leq k-1$, we have

$$
\left\|q_{(\mathbb{A}, \Psi)}(t)\right\|_{L_{l}^{2}} \leq C_{l}\left\|\nabla \mathcal{C}_{\rho}(A(t), \psi(t))\right\|_{L_{l}^{2}}
$$

in the $L_{l}^{2}$-norm on $Y \times\{t\}$, where

$$
\nabla \mathcal{C}_{\rho}(A(t), \psi(t))=\left(-\partial_{A(t)} \psi(t), \sigma(\psi(t), \psi(t))-* F_{A(t)}+2 i \rho\right)
$$

is the gradient flow of the functional $\mathcal{C}_{\rho}$, and $0<C_{l}<1$.

(5) The inequality

$$
\left\|\mathcal{D} q_{(\mathbb{A}, \Psi)}(\alpha(t), \phi(t))\right\| \leq C_{(\mathbb{A}, \Psi)}\left\|\nabla \mathcal{C}_{\rho}(A(t), \psi(t))\right\| \cdot\|(\alpha(t), \psi(t))\|
$$

holds for $t \geq T_{0}$. Here $\mathcal{D} q_{(\mathbb{A}, \Psi)}$ is the linearization of the perturbation $q_{(\mathbb{A}, \Psi)}$.

With a perturbation in the class $\mathcal{P}$ the equations (18) and (19) are invariant with respect to the action of $\mathbb{R}$ by translations along the gradient flow lines, that is if $(A(t), \psi(t))$ is a solution of (18) and (19), then $(A(t+$ $T), \psi(t+T))$ is also a solution for any $T \in \mathbb{R}$.

An example of perturbation with these properties has been constructed by Froyshov [25].

Proposition 2.11. The class of perturbations introduced by Froyshov in $[25]$ is contained in our class $\mathcal{P}$.

According to Froyshov's construction, for fixed smooth compactly supported functions $\eta_{1}, \eta_{2}$, with $\operatorname{supp}\left(\eta_{1}\right) \subset[-1,1]$ and $\left.\eta_{2}\right|_{I}(t)=t$ on an interval $I$ containing all the critical values of $\mathcal{C}_{\rho}$, a function $h: \mathcal{B}_{k, \delta}(a, b) \rightarrow \mathcal{C}^{m}(\mathbb{R})$ is defined as

$$
h_{(\mathbb{A}, \Psi)}(T)=\int_{\mathbb{R}} \eta_{1}(s-T) \eta_{2}\left(\int_{\mathbb{R}} \eta_{1}(t-s) \mathcal{C}_{\rho}(A(t), \psi(t)) d t\right) d s .
$$

Let $\Omega_{\Xi}^{2}(Y \times \mathbb{R})$ be the set of $\mathcal{C}^{m} 2$-forms $\omega$ that are compactly supported in $Y \times \Xi$, where $\Xi$ is the complement of a union of small intervals centered at the critical values of $\mathcal{C}_{\rho}$. Froyshov's perturbation is constructed by setting

$$
P_{(\mathbb{A}, \Psi)}=\left(h_{(\mathbb{A}, \Psi)}^{*}(\omega)\right)^{+},
$$


where $h_{(\mathbb{A}, \Psi)}^{*}(\omega)$ is the pullback of $\omega$ along the map $I d_{Y} \times h_{(\mathbb{A}, \Psi)}: Y \times \mathbb{R} \rightarrow$ $Y \times \mathbb{R}$.

As shown in [25], the function $h_{(\mathbb{A}, \Psi)}$ is bounded with all derivatives, uniformly with respect to $(\mathbb{A}, \Psi)$. Moreover, by the choice of $\Xi$, the perturbation $h_{(\mathbb{A}, \Psi)}^{*}(\omega)$ is smooth and compactly supported, hence in $L_{k, \delta}^{2}$.

Condition (1) holds, since the function $h_{(\mathbb{A}, \Psi)}(t)$ satisfies

$$
h_{(\mathbb{A}, \Psi)}(t+\tau)=h_{(\mathbb{A}, \Psi)^{\tau}}(t),
$$

where $(\mathbb{A}, \Psi)^{\tau}$ is the $\tau$-reparameterized solution represented in a temporal gauge by $(A(t+\tau), \psi(t+\tau))$. In fact,

$$
\begin{aligned}
h_{(\mathbb{A}, \Psi) \tau}(T) & =\int_{\mathbb{R}} \eta_{1}(s-T) \eta_{2}\left(\int_{\mathbb{R}} \eta_{1}(t-s) \mathcal{C}_{\rho}(A(t+\tau), \psi(t+\tau)) d t\right) d s \\
& =\int_{\mathbb{R}} \eta_{1}(s-T) \eta_{2}\left(\int_{\mathbb{R}} \eta_{1}(u-s-\tau) \mathcal{C}_{\rho}(A(u), \psi(u)) d u\right) d s \\
& =\int_{\mathbb{R}} \eta_{1}(v-T-\tau) \eta_{2}\left(\int_{\mathbb{R}} \eta_{1}(u-v) \mathcal{C}_{\rho}(A(u), \psi(u)) d u\right) d v \\
& =h_{(\mathbb{A}, \Psi)}(T+\tau) .
\end{aligned}
$$

Condition (2) holds: in fact, it is shown in [25] that the function $h_{(\mathbb{A}, \Psi)}$ is bounded with all derivatives, uniformly with respect to $(\mathbb{A}, \Psi)$. The Sobolev embeddings of Proposition 2.9 provide the uniform bound in the $L_{k, \delta}^{2}$-norms.

Condition (3) and (5): we can write the function $h_{(\mathbb{A}, \Psi)}$ with the notation

$$
h_{(\mathbb{A}, \Psi)}=\eta_{1} *\left(\eta_{2}\left(\eta_{1} * \mathcal{C}_{\rho}(A, \psi)\right)\right),
$$

where $*$ denotes the convolution product on $\mathbb{R}$. We obtain the variation with respect to $(\mathbb{A}, \Psi)$ of the form

$$
v_{(\mathbb{A}, \Psi)}(\alpha, \phi)=\eta_{1} *\left(\eta_{2}^{\prime}\left(\eta_{1} * \mathcal{C}_{\rho}(A, \psi)\right) \eta_{1} *\left\langle\nabla \mathcal{C}_{\rho}(A, \psi),(\alpha, \phi)\right\rangle\right) .
$$

Thus, as shown in Froyshov ([25], Prop.5), for $\omega$ a $\mathcal{C}^{m}$ form, the linearization of the perturbation $h_{(\mathbb{A}, \Psi)}^{*}(\omega)$ at the point $(\omega, \mathbb{A}, \Psi)$ is a bounded operator $K_{(\omega, \mathbb{A}, \Psi)}: L_{k, \delta}^{2} \rightarrow \mathcal{C}^{m}$ with

$$
\operatorname{supp}\left(K_{(\omega, \mathbb{A}, \Psi)}(\alpha, \Phi)\right) \subset h_{(\mathbb{A}, \Psi)}^{-1}(\Xi) \times Y .
$$

Thus Condition (3) follows, since $\eta_{1}$ and $\eta_{2}$ are smooth compactly supported functions. In particular, if $\omega$ is a smooth form, the linearization $\mathcal{D} P_{(\mathbb{A}, \Psi)}$ is a smoothing operator. The expression of the variation $v_{(\mathbb{A}, \Psi)}$ also shows that Condition (5) holds. 
Condition (4) follows from an estimate on $\left\|d h_{(\mathbb{A}, \Psi)}\right\|$,

$$
\left\|d h_{(\mathbb{A}, \Psi)}(t)\right\| \leq C\left\|\nabla \mathcal{C}_{\rho}(A(t), \psi(t))\right\|,
$$

where the constant $C$ only depends on the asymptotic values $a$ and $b$ of $(\mathbb{A}, \Psi)$.

Other perturbations of the functional $\mathcal{C}$ can be used to achieve transversality of the moduli space of flow lines. For instance see the discussion in [29], and the class of perturbations introduced in [11]. The perturbations introduced in [11] have the advantage of being defined directly as perturbations of the Chern-Simons-Dirac functional $\mathcal{C}$, instead of being perturbations of the 4-dimensional Seiberg-Witten equations, as in the case of the class considered here.

\subsection{Transversality of $\mathcal{M}(a, b)$.}

Let $\mathcal{L}_{(\mathbb{A}, \Psi)}$ be the linearization of equations $(20)$ and $(21)$ on $\mathcal{B}_{k, \delta}(a, b)$.

The operator $\mathcal{L}$ is of the form

$$
\mathcal{L}_{(\mathbb{A}, \Psi, P)}(\alpha, \Phi)=\left\{\begin{array}{l}
D_{\mathbb{A}} \Phi+\alpha \Psi \\
d^{+} \alpha-\frac{1}{2} \operatorname{Im}(\Psi \cdot \bar{\Phi})+\mathcal{D} P_{(\mathbb{A}, \Psi)}(\alpha, \Phi) \\
G_{(\mathbb{A}, \Psi)}^{*}(\alpha, \Phi)
\end{array}\right.
$$

mapping

$$
\begin{aligned}
& L_{k, \delta}^{2}\left(\Omega^{1}(Y \times \mathbb{R}, i \mathbb{R}) \oplus \Gamma\left(S^{+} \otimes L\right)\right) \\
& \quad \rightarrow L_{k-1, \delta}^{2}\left(\Omega^{0}(Y \times \mathbb{R}, i \mathbb{R}) \oplus \Omega^{2+}(Y \times \mathbb{R}, i \mathbb{R}) \oplus \Gamma\left(S^{-} \otimes L\right)\right) .
\end{aligned}
$$

The operator $G^{*}$ is the adjoint, in the $L_{0, \delta}^{2}$-pairing, of the linearization of the gauge group action $G_{(\mathbb{A}, \Psi)}(f)=(-d f, f \Psi)$.

As the following proposition shows, the operator $\mathcal{L}_{(\mathbb{A}, \Psi, P)}$ is obtained by adding the compact perturbation $\mathcal{D} P_{(\mathbb{A}, \Psi)}$ to a Fredholm map from $L_{k, \delta}^{2}$ to $L_{k-1, \delta}^{2}$, hence it is Fredholm. Therefore we have a well defined relative Morse index of two critical points $a$ and $b$ in $\mathcal{M}$.

Proposition 2.12. Suppose $a$ and $b$ are irreducible critical points for the functional $\mathcal{C}_{\rho}$. Let $\left\{\lambda_{a}\right\}$ and $\left\{\lambda_{b}\right\}$ be the eigenvalues of the Hessian $T$ at the points $a$ and $b$. Assume that the positive number $\delta$ satisfies the condition

$$
\delta<\min \left\{\left|\lambda_{a}\right|,\left|\lambda_{b}\right|\right\}
$$


Let $(\mathbb{A}, \Psi)$ be a solution of $(20)$ and $(21)$ in $\mathcal{B}_{k, \delta}(a, b)$. Then the linearization $\mathcal{L}_{(\mathbb{A}, \Psi)}$ is a Fredholm operator of index

$$
\operatorname{Ind}\left(\mathcal{L}_{(\mathbb{A}, \Psi)}\right)=\sigma(a, b) .
$$

The right hand side $\sigma(a, b)$ is the spectral flow of the operator $\nabla \mathcal{F}$ along a path $(A(t), \psi(t))$ in $\mathcal{A}$ that corresponds to $(\mathbb{A}, \Psi)$ under $\pi^{*}$. The quantity $\sigma(a, b)$ is independent of the path, hence

$$
\sigma(a, b)=\mu(a)-\mu(b)
$$

defines a relative Morse index of $a$ and $b$, where $\mu(a)$ is the spectral flow of $\nabla \mathcal{F}$ on a path joining a to a fixed $\left[A_{0}, \psi_{0}\right]$ in $\mathcal{M}$.

Remark 2.13. In the case with $Y$ a homology sphere, Proposition 2.12 holds for a reducible point $a=[\nu, 0]$ under the assumption that, for the chosen metric $g$ on $Y$, the condition $\operatorname{Ker}\left(\partial_{\nu}^{g}\right)=0$ is satisfied.

We state and prove an analogue of Proposition 2.12 in the context of framed moduli spaces and equivariant theory in Theorem 3.8. We also prove, in Theorem 3.8, that the relative Morse index of points in $\tilde{\mathcal{M}}$ is well defined.

Consider the moduli space $\mathcal{M}(a, b)$ of solutions of the equations (20) and $(21)$ in $\mathcal{B}_{k, \delta}(a, b)$.

Proposition 2.14. When $a$ and $b$ are irreducible critical points of $\mathcal{C}_{\rho}$, for a generic choice of the perturbation $P \in \mathcal{P}$, the moduli space $\mathcal{M}(a, b)$ of gradient flow lines is a smooth oriented manifold, cut out transversely by the equations, of dimension

$$
\operatorname{dim}(\mathcal{M}(a, b))=\mu(a)-\mu(b),
$$

where $\mu(a)-\mu(b)$ is the relative Morse index of the critical points.

Proof. It is first necessary to know that there are no reducible flow lines connecting the critical points $a$ and $b$. This fact is an easy consequence of the definition of the configuration space (17), since the exponential weight in the Sobolev norm forces elements in (17) to decay at the ends to the asymptotic values, which are irreducible by assumption. On the convergence of flow lines to the endpoints $a$ and $b$, see the results of Section 3.4. Thus, $\mathcal{M}(a, b)$ lies entirely in the irreducible component $\mathcal{B}_{k, \delta}^{\prime}(a, b)$, provided that at least one of the endpoints $a$ and $b$ is irreducible. The statement then 
follows via the implicit function theorem, upon showing that, for a generic choice of the perturbation $P$, the linearization $\mathcal{L}$ is surjective.

Consider the operator

$$
\hat{\mathcal{L}}_{(\mathbb{A}, \Psi, P)}(\alpha, \Phi, p)=\mathcal{L}_{(\mathbb{A}, \Psi, P)}(\alpha, \Phi)+p_{(\mathbb{A}, \Psi, P)}(\alpha, \Phi),
$$

where we vary the perturbation by an element $p_{(\mathbb{A}, \Psi, P)}$ of $T_{P} \mathcal{P}$. This corresponds to varying the parameter $\omega \in \Omega_{\Xi}^{2}(Y \times \mathbb{R})$ in Froyshov's class of perturbations.

The operator $\mathcal{L}$ is Fredholm, therefore $\hat{\mathcal{L}}$ has a closed range. We show that $\hat{\mathcal{L}}$ is surjective by proving that it has dense range.

Suppose given an element $(\beta, \xi, g)$ in $L_{-k-1,-\delta}^{2}$ that is $L^{2}$-orthogonal to the range of the operator $\hat{\mathcal{L}}$. Here $\beta$ is an element in $\Omega^{2+}(Y \times \mathbb{R}, i \mathbb{R}), \xi$ is a spinor, and $g$ is a zero-form. The element $(\beta, \xi, g)$ is in the kernel of the adjoint $\tilde{\mathcal{L}}^{*}$, which is an elliptic operator with $L_{-k,-\delta}^{2}$ coefficients, thus $(\beta, \xi)$ lives in $L_{-k,-\delta}^{2}$ by elliptic regularity. In fact, the perturbation satisfies Condition (3) of Definition 2.10. If we consider the $L^{2}$-pairing of $L_{k, \delta}^{2}$ and $L_{-k,-\delta}^{2}$, we get

$$
\begin{aligned}
\left\langle\beta, d^{+} \alpha-\frac{1}{2} \operatorname{Im}(\Psi \cdot \bar{\Phi})+\mathcal{D} P_{(\mathbb{A}, \Psi)}(\alpha, \Phi)+p_{(\mathbb{A}, \Psi, P)}(\alpha, \Phi)\right\rangle \\
+\left\langle\xi, D_{\mathbb{A}} \Phi+\alpha \Psi\right\rangle+\left\langle g, G_{(\mathbb{A}, \Psi)}^{*}(\alpha, \Phi)\right\rangle=0 .
\end{aligned}
$$

By varying $p \in \mathcal{P}$ we force $\beta \equiv 0$. The remaining inner product

$$
\left\langle\xi, D_{\mathbb{A}} \Phi+i \alpha \Psi\right\rangle+\left\langle g, G_{(\mathbb{A}, \Psi)}^{*}(\alpha, \Phi)\right\rangle=0
$$

gives the following equations

(a) $\left(e_{-\delta} d e_{\delta}\right) g=\frac{1}{2} \xi \cdot \bar{\Psi}$ and

(b) $D_{A} \xi-g \Psi=0$.

We assume that $\Psi$ is not identically zero. Applying $d^{*}$ to (a) and using (b) we obtain $d^{*}\left(e_{-\delta} d e_{\delta} g\right)+g|\Psi|^{2}=0$. Equivalently, we get

$$
\left(e_{\delta / 2} d^{*} e_{-\delta / 2}\right)\left(e_{-\delta / 2} d e_{\delta / 2}\right) e_{\delta / 2} g+|\Psi|^{2} e_{\delta / 2} g=0 .
$$

The equation

$$
\Delta_{\delta / 2} e_{\delta / 2} g+e_{\delta / 2} g|\Psi|^{2}=0
$$

with

$$
\Delta_{\delta}=e_{-\delta} \Delta e_{\delta}
$$


implies that $g \equiv 0$, since $g$ decays at $\pm \infty$ and the maximum principle applies. Then, by varying $\alpha$ alone in $\left\langle\xi, D_{\mathbb{A}} \Phi+\alpha \Psi\right\rangle=0$, we force $\xi$ to vanish on some arbitrary open set. We obtain $(\beta, \xi) \equiv 0$.

Thus the operator $\tilde{\mathcal{L}}$ is surjective. This implies that zero is a regular value for the map defined by the equations (20) and (21). Therefore the moduli space $\mathcal{M} o d$ of triples $([\mathbb{A}, \Psi], P)$ in $\mathcal{B}_{L_{k, \delta}^{2}}^{\prime}(a, b) \oplus \mathcal{P}$ that satisfy the equations is a smooth (infinite dimensional) manifold with tangent space $\operatorname{Ker}(\hat{\mathcal{L}})$.

The projection $\Pi: \mathcal{M} o d \rightarrow \mathcal{P}$ given by $\Pi([\mathbb{A}, \Psi], P)=P$ linearizes to a surjective Fredholm operator $\mathcal{D} \Pi: \operatorname{Ker}(\tilde{\mathcal{L}}) \rightarrow T_{P} \mathcal{P}$. The kernel of $\mathcal{D} \Pi$ is $\operatorname{Ker}\left(\mathcal{D} \Pi_{(\mathbb{A}, \Psi, P)}\right)=\operatorname{Ker}\left(\mathcal{L}_{(\mathbb{A}, \Psi, P)}\right)$. The infinite dimensional Sard theorem implies that the moduli space $\mathcal{M}(a, b)$, for a generic perturbation $P \in \mathcal{P}$, is the inverse image under the projection map from $\mathcal{M o d}$ to $\mathcal{P}$ of a regular value. Thus $\mathcal{M}(a, b)$ is a smooth manifold which is cut out transversely by the equations. Equivalently, the linearization $\mathcal{L}$ with a fixed generic $q$ is surjective.

The virtual dimension of the moduli space $\mathcal{M}(a, b)$ equals the index of the Fredholm operator $\mathcal{L}$. According to Proposition 2.12, this is the relative Morse index $\mu(a)-\mu(b)$.

The orientation of $\mathcal{M}(a, b)$ is given by a trivialization of the determinant line bundle of the operator $\mathcal{L}$. This is obtained given a choice of an orientation of

$$
H_{\delta}^{0}(Y \times \mathbb{R}) \oplus H_{\delta}^{2+}(Y \times \mathbb{R}) \oplus H_{\delta}^{1}(Y \times \mathbb{R}),
$$

the cohomology groups of $\delta$-decaying forms, as discussed in the following Proposition.

Proposition 2.15. The manifold $\mathcal{M}(a, b)$ is oriented by a trivialization of the determinant line bundle of the operator $\mathcal{L}$. This is obtained from an orientation of

$$
H_{\delta}^{0}(Y \times \mathbb{R}) \oplus H_{\delta}^{2+}(Y \times \mathbb{R}) \oplus H_{\delta}^{1}(Y \times \mathbb{R}),
$$

the cohomology groups of $\delta$-decaying forms.

Proof. Suppose given $[x]=[\mathbb{A}, \Psi] \in \mathcal{M}(a, b)$. Let $x=(A(t), \psi(t))$ be a temporal gauge representative such that

$$
\lim _{t \rightarrow \infty}(A(t), \psi(t))=\left(A_{b}, \psi_{b}\right)
$$


and

$$
\lim _{t \rightarrow-\infty}(A(t), \psi(t))=\left(A_{a}, \psi_{a}\right)
$$

Consider the family of operators

$$
\mathcal{L}_{x}(\alpha, \Phi)=\left\{\begin{array}{l}
d^{+} \alpha-\frac{1}{2} \operatorname{Im}(\Psi \cdot \bar{\Phi}) \\
D_{\mathbb{A}} \Phi+\alpha \Psi \\
G_{(\mathbb{A}, \Psi)}^{*}(\alpha, \Phi)
\end{array}\right.
$$

acting on the space of $L_{k, \delta}^{2}$-decaying 1-forms and spinor sections on $Y \times \mathbb{R}$.

An orientation of the moduli space $\mathcal{M}(a, b)$ is determines by a trivialization of the determinant line bundle of the family of operators $\mathcal{L}_{x}$. We can separate $\mathcal{L}_{x}$ in the first order term and a perturbation,

$$
\mathcal{L}_{x}=\mathcal{L}_{x}^{1}+\mathcal{L}_{x}^{0}
$$

with

$$
\mathcal{L}_{x}^{1}=d^{+}+d_{\delta}^{*}+D_{\mathbb{A}}
$$

and

$$
\mathcal{L}_{x}^{0}=\left(\begin{array}{c}
-\frac{1}{2} \operatorname{Im}(\cdot \bar{\Psi}) \\
i\langle\Psi, \cdot\rangle
\end{array}\right) .
$$

The operators induced by $\mathcal{L}_{x}$ on the asymptotic ends are

$$
L( \pm \infty)=L^{1}( \pm \infty)+L^{0}( \pm \infty)
$$

where we have

$$
\begin{aligned}
L^{1}(\infty) & =\left(\begin{array}{ccc}
* d & d^{*} & 0 \\
d & 0 & 0 \\
0 & 0 & \partial_{A_{b}}
\end{array}\right), \\
L^{1}(-\infty) & =\left(\begin{array}{ccc}
* d & d^{*} & 0 \\
d & 0 & 0 \\
0 & 0 & \partial_{A_{a}}
\end{array}\right)
\end{aligned}
$$

and

$$
\begin{aligned}
L^{0}(\infty) & =\left(\begin{array}{ccc}
0 & 0 & -2 i \operatorname{Im} \sigma\left(\cdot, \psi_{b}\right) \\
0 & 0 & -i<\cdot, \psi_{b}> \\
\cdot \psi_{b} & \psi_{b} & 0
\end{array}\right), \\
L^{0}(-\infty) & =\left(\begin{array}{ccc}
0 & 0 & -2 i \operatorname{Im} \sigma\left(\cdot, \psi_{a}\right) \\
0 & 0 & -i<\cdot, \psi_{a}> \\
\cdot \psi_{a} & \psi_{a} & 0
\end{array}\right)
\end{aligned}
$$


acting on $\Omega^{0}(Y, i \mathbb{R}) \oplus \Omega^{1}(Y, i \mathbb{R}) \oplus \Gamma(S \otimes L)$.

We can consider a deformation $\mathcal{L}_{x}^{\epsilon}$ of the family $\mathcal{L}_{x}$ obtained as in [45] Section 6.6,

$$
\mathcal{L}_{x}^{\epsilon}=\mathcal{L}_{x}^{1}+(1-\epsilon) \mathcal{L}_{x}^{0} .
$$

The deformation changes the asymptotic operators in the form

$$
L^{\epsilon}( \pm \infty)=L^{1}( \pm \infty)+(1-\epsilon) L^{0}( \pm \infty),
$$

We can guarantee that this is a deformation via Fredholm operators provided that the weight $\delta$ is chosen such that $\delta / 2$ is not in the spectrum of $L^{\epsilon}( \pm \infty)$ for all $\epsilon$ ([36] Theorem 6.2, and [47] Lemma 8.3.1). That is, if the spectrum of the operators $L^{\epsilon}( \pm \infty)$ is uniformly bounded away from zero.

If this is the case, then a trivialization of the determinant line of the family $\mathcal{L}_{x}^{\epsilon}$ is obtained by a trivialization at $\epsilon=1$. This induces a trivialization of $\mathcal{L}_{x}$. The trivialization at $\epsilon=1$ is a trivialization of the determinant line of the operator $d^{+}+d_{\delta}^{*}+D_{\mathbb{A}}$.

The Dirac operator is complex linear and it preserves the orientation induced by the complex structure on the spinor bundle $S^{+} \otimes L$. Thus a trivialization is obtained by an orientation of

$$
H_{\delta}^{0}(Y \times \mathbb{R}) \oplus H_{\delta}^{2+}(Y \times \mathbb{R}) \oplus H_{\delta}^{1}(Y \times \mathbb{R}),
$$

the cohomology groups of $\delta$-decaying forms, [45], [48].

However, the condition on the spectrum of $L^{\epsilon}( \pm \infty)$ may not always be satisfied: the deformation $\mathcal{L}_{x}^{\epsilon}$ may not be through Fredholm operators. It is still possible to obtain an orientation of $\mathcal{M}(a, b)$ : the following argument was suggested to us by L. Nicolaescu [51].

We can change the family $\mathcal{L}_{x}$ by a deformation such that the new family $\mathcal{H}_{x}$ satisfies

$$
\left.\left.\mathcal{H}_{x}\right|_{Y \times[-1,1]} \equiv \mathcal{L}_{x}\right|_{Y \times[-1,1]} .
$$

On $Y \times(-\infty,-2] \cup Y \times[2, \infty)$ it satisfies

$$
\begin{gathered}
\left.\mathcal{H}_{x}\right|_{Y \times(-\infty,-2]}=d^{+}+d_{\delta}^{*}+D_{A_{a}}+\left(\begin{array}{c}
-\frac{1}{2} \operatorname{Im}\left(\cdot \bar{\psi}_{a}\right) \\
i\left\langle\psi_{a}, \cdot\right\rangle
\end{array}\right), \\
\left.\mathcal{H}_{x}\right|_{Y \times[2, \infty)}=d^{+}+d_{\delta}^{*}+D_{A_{b}}+\left(\begin{array}{c}
-\frac{1}{2} \operatorname{Im}\left(\cdot \bar{\psi}_{b}\right) \\
i\left\langle\psi_{b}, \cdot\right\rangle
\end{array}\right) .
\end{gathered}
$$

A trivialization of the determinant line of $\mathcal{H}_{x}$ induces a trivialization of the determinant of $\mathcal{L}_{x}$. 
The index of the family $\operatorname{Ind}\left(\mathcal{H}_{x}\right)$ on $Y \times \mathbb{R}$ equals the index on $Y \times[-3,3]$ with APS boundary conditions [3], [53].

Now we can consider the family of operators $\tilde{\mathcal{H}}_{x}$ that satisfies

$$
\left.\left.\tilde{\mathcal{H}}_{x}\right|_{Y \times[-3,3]} \equiv \mathcal{H}_{x}\right|_{Y \times[-3,3]} .
$$

On $Y \times(-\infty,-4] \cup Y \times[4, \infty)$ it satisfies

$$
\begin{gathered}
\left.\tilde{\mathcal{H}}_{x}\right|_{Y \times(-\infty,-4]}=d^{+}+d_{\delta}^{*}+D_{A_{a}}, \\
\left.\tilde{\mathcal{H}}_{x}\right|_{Y \times[4, \infty)}=d^{+}+d_{\delta}^{*}+D_{A_{b}} .
\end{gathered}
$$

On the cylinder $Y \times[-4,-3]$ and $Y \times[3,4]$ the index of the operator $\tilde{\mathcal{H}}_{x}$ can be obtained as $\operatorname{Ind}\left(\frac{\partial}{\partial t}+H(t)\right)$, where $H(t)$ is independent of $x$ (but depends on the asymptotic values $a$ or $b)$. Thus, $\operatorname{Ind}\left(\tilde{\mathcal{H}}_{x}\right)$ on $Y \times[-4,-3]$ and $Y \times[3,4]$ is the spectral flow $S F(H(t))$ of $H(t)$.

The indices of $\mathcal{H}_{x}$ and $\tilde{\mathcal{H}}_{x}$ are related by the excision formula

$$
\operatorname{Ind}\left(\mathcal{H}_{x}\right)-S F(H(t))=\operatorname{Ind}\left(\tilde{\mathcal{H}}_{x}\right),
$$

generalizing the excision formula of [52].

Thus the relative orientation of $\mathcal{H}_{x}$ and by $\tilde{\mathcal{H}}_{x}$ is exactly $(-1)^{S F(H)}$. Notice that [52] provides examples where this spectral flow is computed explicitly and is odd.

Finally we can introduce a deformation $\tilde{\mathcal{H}}_{x}^{\epsilon}$ with a homotopy that shrinks to zero the spinor part. In this case the asymptotic operators $H( \pm \infty)$ remain constant, hence the deformation is through Fredholm operators. This implies that the orientation determined by $\tilde{\mathcal{H}}_{x}$ is the same as the one determined by $d^{+}+d_{\delta}^{*}+D_{\mathbb{A}}$, that is by an orientation of

$$
H_{\delta}^{0}(Y \times \mathbb{R}) \oplus H_{\delta}^{2+}(Y \times \mathbb{R}) \oplus H_{\delta}^{1}(Y \times \mathbb{R}) .
$$

\subsection{Floer homology.}

The Floer complex has generators

$$
C_{q}=\left\{b \in \mathcal{M}^{\prime} \mid \mu(b)=q\right\},
$$

where $\mathcal{M}^{\prime}$ is the irreducible part of the moduli space of critical points. The Morse index $\mu(b)$ is computed with respect to a fixed element $\left[A_{0}, \psi_{0}\right]$ in $\mathcal{M}$, $\left[A_{0}, \psi_{0}\right]=[\nu, 0]$ in the case of a homology sphere. 
The boundary operator is given by

$$
\partial a=\sum_{b \mid \mu(a)-\mu(b)=1} \epsilon(a, b) b,
$$

where $\epsilon(a, b)$ is the algebraic sum over the paths joining $a$ and $b$ of the signs given by the orientation,

$$
\epsilon(a, b)=\sum_{\gamma \in \hat{\mathcal{M}}(a, b)} \epsilon_{\gamma}
$$

Here $\mathcal{M}(a, b)$ is the moduli space of flow lines on $Y \times \mathbb{R}$ with asymptotic values $a$ and $b$. A description of $\mathcal{M}(a, b)$ will be given in the next section. The space $\hat{\mathcal{M}}(a, b)$ is the quotient of $\mathcal{M}(a, b)$ by the action of $\mathbb{R}$ by translations. A compactness result for $\hat{\mathcal{M}}(a, b)$ is needed in order to make sense of $\epsilon(a, b)$. This result will follow from the more general result proved in Section 4 in the equivariant setup, in Theorem 4.1, Theorem 4.9, and Proposition 4.25 .

The property that $\partial \circ \partial=0$ relies on the gluing formula of Lemma 2.16 that follows from an accurate analysis of the properties of the gradient flow moduli space $\mathcal{M}(a, b)$. In our setting, again, this result will follow from the more general results in the equivariant context, see Theorem 4.9.

In the case where $Y$ is a homology sphere, we need to ensure that in the expression of $\partial^{2}$ there is no contribution coming from trajectories that break through the unique reducible solution. In other words, no component of the form $\hat{\mathcal{M}}(a, \theta) \times \hat{\mathcal{M}}(\theta, b)$ can appear in the boundary of $\hat{\mathcal{M}}(a, b)$ for $\mu(a)-\mu(b)=2$. This has been proved in [13]. In fact, the following gluing formula holds.

Lemma 2.16. Suppose given $a, b$ and $c$ in $\mathcal{M}^{\prime}$, irreducible critical points with $\mu(a)>\mu(b)>\mu(c)$. Then, for large enough $T$, there is a local diffeomorphism

$$
\hat{\mathcal{M}}(a, b) \times \hat{\mathcal{M}}(b, c) \times[T, \infty) \rightarrow \hat{\mathcal{M}}(a, c) .
$$

If $Y$ is a homology sphere and $\theta=[\nu, 0]$ is the unique reducible critical point, there is a local diffeomorphism

$$
\hat{\mathcal{M}}(a, \theta) \times \hat{\mathcal{M}}(\theta, c) \times U(1) \times[T, \infty) \rightarrow \hat{\mathcal{M}}(a, c) .
$$

In this case $U(1)$ is the stabilizer of the reducible solution $\theta=[\nu, 0]$. 
In Theorem 4.9, we prove the gluing formula in the equivariant setup. A proof of Lemma 2.16 can be found in [38] and [13], and it follows from our equivariant result, as discussed at the end of Section 4.2. The result has an immediate corollary.

Corollary 2.17. Suppose $Y$ is a homology sphere. Let $\theta$ be the unique reducible solution. If $a$ and $c$ are irreducible critical points such that $\mu(a)-$ $\mu(c)=2$, then generically there will be no boundary strata of the form $\hat{\mathcal{M}}(a, \theta) \times \hat{\mathcal{M}}(\theta, c)$. In fact for dimensional reasons the moduli space $\hat{\mathcal{M}}(\theta, c)$ of gradient flow lines is generically empty if $\mu(\theta)-\mu(c)=1$.

The property that $\partial^{2}=0$ in the Floer complex follows then from the fact that the matrix elements

$$
\langle\partial \partial a, c\rangle=\sum_{b}\langle\partial a, b\rangle\langle\partial b, c\rangle,
$$

of the operator $\partial^{2}$ are the algebraic sum of the points of the oriented zerodimensional manifold

$$
\cup_{b \in \mathcal{M}^{\prime}} \hat{\mathcal{M}}(a, b) \times \hat{\mathcal{M}}(b, c)=\partial \hat{\mathcal{M}}(a, c) .
$$

In the case with non-trivial $b^{1}(Y)$, we construct the Floer homology under the assumption that $c_{1}(L) \neq 0$ rationally and that the perturbation is restricted to the trivial cohomology class $[* \rho]=0$.

Remark 2.18. For a 3-manifold $Y$ with $b_{1}>0$ and a $\operatorname{Spin}_{c}$-structure with $c_{1}(L)=0$ rationally, the methods discussed here do not extend directly to formulate the corresponding monopole homology. In fact, the ChernSimons-Dirac functional is $\mathbb{R}$-valued on the configuration space as long as the perturbation term represents a trivial de Rham cohomology class. In this case, however, the condition $c_{1}(L)=0$ implies the existence of a reducible set of critical points that is a torus $T^{b_{1}(Y)}$. These can be degenerate, in the sense of Morse-Bott, even in the framed configuration space. Perturbing the functional with a 1 -form $\rho$ that is non-trivial cohomologically can destroy this reducible set, but the functional would no longer be $\mathbb{R}$-valued. There is then no uniform energy bound on the space of flow lines of a fixed virtual dimension. This creates a problem in the compactification by broken trajectories (see Theorem 4.1 and Theorem 4.9). The right framework for this bad case seems to be a Novikov type complex, where trajectories with the same virtual dimension but with different energies are counted separately as coefficients of a power series. Since this case has important applications in the gluing formulae, we deal with it separately in [42]. 
Under our assumptions, when we have $b^{1}(Y)>1$ and a nontrivial $c_{1}(L)$, the Floer homology groups can be proved to be independent of the metric and of the perturbation. In fact a chain map and a chain homotopy are constructed by a cobordism argument between moduli spaces for two different metrics and perturbations. This result follows from the topological invariance of the equivariant Floer homology proved in Theorem 6.1, in Section 6, and the equivalence of equivariant and non-equivariant Floer theories in the case of manifolds with $b^{1}(Y)>1$ and a nontrivial $c_{1}(L)$, proved in Section 7, Theorem 7.1.

In the case with $b^{1}(Y)=1$ one expects to find a dependence on the choice of the perturbation, see [35], however, since we are only considering perturbations that are cohomologically trivial, we obtain independence of the metric and perturbation as in the $b^{1}(Y)>1$ case. A similar dependence was detected in [5] in the case of the invariant of three-manifolds obtained by counting points in $\tilde{\mathcal{M}}$ with the orientation. In [38] it is proved in the case $b^{1}(Y)>0$ that this invariant is in fact the Euler characteristic of the Floer homology. The same invariant was introduced in [12] following the Quantum Field Theory formulation of Seiberg-Witten theory.

In the case of a homology sphere the metric dependence problem is more complicated. In fact due to the reducible solution a cobordism argument does not work and more generally the construction of a chain homotopy can fail due to the presence of moduli spaces of gradient flow lines that connect the irreducibles to the reducible critical point. The space of metrics and perturbations breaks into chambers with codimension-one walls, so that the Floer groups are isomorphic for metrics that belong to the same chamber and are in general non-isomorphic when the metric crosses a wall. We shall discuss the wall-crossing phenomenon in Section 7.

\section{Morse-Bott theory.}

We are now going to introduce the equivariant Floer complex. This can be constructed for all three-manifolds. Clearly in the case of an integral

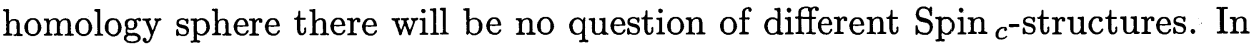
the case of a rational homology sphere, we have finitely many choices of $\operatorname{Spin}_{c}$-structures and there is a reducible point corresponding to each of these. In the case of manifolds with $b^{1}(Y)>0$ there are infinitely many possible choices of $\operatorname{Spin}_{c}$-structures. This gives rise to a family of Floer complexes corresponding to the different choices of the $\operatorname{Spin}_{c}$-structures. The relation between these requires further investigation and is analyzed 
elsewhere (cf. [11]). In all the following we always assume to work with a fixed choice of the $\operatorname{Spin}_{c}$-structure.

In order to consider reducible as well as irreducible generators, we introduce a framed configuration space with a $U(1)$-action, where the functional $\mathcal{C}_{\rho}$ is defined as a $U(1)$-invariant real valued functional. In order to apply the analogue of the finite dimensional equivariant Morse theory [7], we need $\mathcal{C}$ to be a Morse-Bott function. That is, we have to ensure that the Hessian is non-degenerate on the normal bundle to the critical $U(1)$-orbits.

\subsection{Framed moduli space.}

Definition 3.1. Let $x_{0}$ be a fixed base point in $Y$. We define the space $\mathcal{B}^{0}$ to be the quotient of $\mathcal{A}$ with respect to the action of the subgroup $\mathcal{G}^{0} \subset \mathcal{G}$ of gauge transformations $\lambda$ that act as the identity on the fiber of $S \otimes L$ over $x_{0}$ and that satisfy the condition (2). The space $\mathcal{B}^{0}$ is the framed configuration space.

The action of the group $\mathcal{G}^{0}$ on $\mathcal{A}$ is free, therefore the space $\mathcal{B}^{0}$ is an infinite dimensional Banach manifold (using a fixed $L_{k}^{2}$ norm) that carries a residual $U(1)$ action. There is a fibration $\mathcal{B}^{0} \rightarrow \mathcal{B}$ over the unframed configuration space with fiber $U(1)$. The solutions of the three dimensional Seiberg-Witten equations (5) and (6) in $\mathcal{B}^{0}$ form the framed moduli space $\mathcal{M}^{0}$, that is the critical set of the functional (3) modulo based gauge transformations.

As in the case of Donaldson theory [16], an equivalent description of the framed configuration space can be given as the triples $(A, \psi, \phi)$ with $(A, \psi) \in \mathcal{A}$ and $\phi$ a unit vector in the fiber $\left.S \otimes L\right|_{x_{0}}$. The full gauge group acts freely on this space. Solutions of the Seiberg-Witten equations in this configuration space modulo the full gauge group provide another model of framed moduli space. This has been used in [4] and [5]. We use the description given in definition 3.1 , since $\mathcal{M}^{0}$ has an explicit $U(1)$ action which allows us to work equivariantly.

Since the action of the base point preserving gauge transformations on $\mathcal{A}$ is free, the reducible solutions with $\psi \equiv 0$ now have trivial stabilizer, hence they are smooth points in $\mathcal{M}^{0}$. The reducible part of the unframed moduli space $\mathcal{M}$ corresponds exactly to the fixed point set for the $U(1)$ action on $\mathcal{M}^{0}$.

Lemma 3.2. Consider the unperturbed equations (9) in $\mathcal{B}^{0}$. Let $[A, 0]$ be a solution that is a fixed point of the U(1)-action. Then the virtual tangent 
space of $\mathcal{M}^{0}$ at the point $[A, 0]$ is $H^{1}(Y, \mathbb{R}) \oplus \operatorname{Ker}\left(\partial_{A}\right)$. Moreover, the set of fixed points in $\mathcal{M}^{0}$ is identified with the torus $H^{1}(Y, \mathbb{R}) / H^{1}(Y, \mathbb{Z})$ together

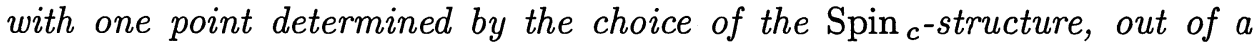
discrete set given by the torsion part of $H^{1}(Y, \mathbb{Z})$.

Proof. Fixed points are flat $U(1)$-connections modulo gauge: these are the representations of $\pi_{1}(Y)$ into $U(1)$. The linearization $T$ at a point $[A, 0]$ is of the form $\left(-* d, \partial_{A}\right)$. For the element in the torsion part of $H^{1}(Y, \mathbb{Z})$ see Theorem 3.4 below.

We need to perturb the equations in some generic way in order to have $\mathcal{M}^{0}$ cut out transversely. For the perturbed equations (15), the fixed point set is described by the equation $* F_{A}=2 i \rho$.

Lemma 3.3. If $Y$ has non-trivial $b^{1}(Y)$ and the functional $\mathcal{C}$ is perturbed with a generic co-closed 1-form $\rho$, then the set of critical orbits $\mathcal{M}^{0}$ contains no fixed point and is cut out transversely by the equations. The Hessian of the perturbed functional $\mathcal{C}_{\rho}$ is non-degenerate in the directions normal to the critical orbits.

Proof. If the Chern class $c_{1}(L)$ is non-trivial, choose a perturbation $\rho$ with $[* \rho] \neq i \pi c_{1}(L)$, or perturb with a harmonic form if $c_{1}(L)=0$. This implies that there are no solutions of the equation $F_{A}=2 i * \rho$.

Consider the linearization $\hat{L}_{(A, \psi, \rho)}$ of the equations (15), where we allow the perturbation to vary,

$$
\hat{L}_{(A, \psi, \rho)}(\alpha, \phi, \eta)=L_{(A, \psi, \rho)}(\alpha, \phi)-2 i \eta .
$$

The operator $\hat{L}$ has closed range, since $L$ is Fredholm. We show that $\hat{L}$ is surjective. Let $(\beta, \xi, g)$ be an element that is $L^{2}$-orthogonal to the range of $\hat{L}$. Then $(\beta, \xi, g)$ is in the kernel of the adjoint, hence by elliptic regularity we can consider the $L^{2}$ pairing of $L_{k}^{2}$ and $L_{-k}^{2}$,

$$
\begin{aligned}
\langle\beta,-* d \alpha-d f+2 \sigma(\psi, \phi)- & 2 i \eta\rangle \\
& +\left\langle\xi, \partial_{A} \phi+\alpha \psi+f \psi\right\rangle+\left\langle g, G^{*}(\alpha, \phi)\right\rangle=0 .
\end{aligned}
$$

The argument is analogous to the proof of Proposition 2.14. By varying $\eta$ we force $\beta \equiv 0$. The vanishing of

$$
\left\langle\xi, \partial_{A} \phi+\alpha \psi+f \psi\right\rangle+\left\langle g, G^{*}(\alpha, \phi)\right\rangle
$$


gives an equation $\Delta g+1 / 2 g|\psi|^{2}=0$ which implies $g \equiv 0$ by the maximum principle. Then by varying $\phi$ and $\alpha$ we get $\partial_{A} \xi=0$ and $\sigma(\xi, \psi)=0$. The latter is satisfied if $\xi$ is an imaginary multiple of $\psi, \xi=i \lambda \psi$, where neither of the two vanishes. Both $\xi$ and $\psi$ are in the kernel of $\partial_{A}$, thus if either of them vanishes on an open set it has to vanish identically (and we know that $\psi$ is not identically zero). If we have $\xi=i \lambda \psi$, we obtain that $\xi$ is identically zero as a consequence of the vanishing of the inner product $\langle\xi, f \psi\rangle$ for arbitrary smooth compactly supported functions $f$.

This is enough to show that for a generic perturbation $\rho$ the moduli space $\mathcal{M}$ (and therefore also $\mathcal{M}^{0}$ ) is cut out transversely, as in the analogous proof of Proposition 2.14 .

When $b^{1}(Y)=0$ the virtual tangent space at a solution of $* F_{A}=2 i * d \nu$ is identified with $\operatorname{Ker}\left(\partial_{A}\right)$ and the perturbation $\rho=* d \nu$ is not enough to ensure that $\mathcal{M}^{0}$ is cut out transversely and that the fixed point set is separated from the other components of $\mathcal{M}^{0}$.

Theorem 3.4. Let $Y$ be a rational homology sphere. Suppose we choose a perturbation by a co-closed 1-form $\rho$ and a generic metric. Then the framed moduli space $\mathcal{M}^{0}$ consists of a disjoint union of finitely many circles (corresponding to the irreducible part of the unframed $\mathcal{M}$ ) and finitely many points (the reducibles of $\mathcal{M}$ ). These correspond to different choices

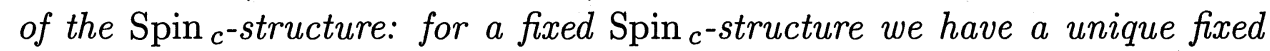
point of the $U(1)$-action. Moreover, the Hessian of the functional $\mathcal{C}_{\rho}$ is nondegenerate in the normal directions to the critical orbits.

Proof. The choice of a perturbation $\rho=* d \nu$ makes the fixed point set into the finitely many solutions modulo gauge of

$$
F_{A}=d \nu,
$$

namely flat $U(1)$ connections modulo gauge. These are representations of the $\pi_{1}(Y)$ into $U(1)$, hence they are identified with the finitely many elements in the group $H^{1}(Y, \mathbb{Z})$. Each of these elements specifies one choice of the Spin ${ }^{c}$-structure. Thus, for fixed Spin ${ }^{c}$-structure, there is a unique fixed point $\theta=\left[A_{0}+\nu, 0\right]$ of the $U(1)$-action in the moduli space $\mathcal{M}^{0}$. If the metric on $Y$ satisfies $\operatorname{Ker}\left(\partial_{A}\right)=0$ at a solution $[A, 0]$ of $(1)$, then the virtual tangent space at this fixed point is zero-dimensional, hence the Hessian of $\mathcal{C}_{\rho}$ is non-degenerate at the fixed points. The condition is satisfied for a generic metric because of Lemma 2.7. 
The irreducible component of $\mathcal{M}$ is a zero dimensional manifold if the perturbation $\rho$ is generic. The moduli space $\mathcal{M}$ is compact, since $b^{1}(Y)=0$, and therefore so is $\mathcal{M}^{0}$. However, we have to show that the unique fixed point is separated from the other components of $\mathcal{M}^{0}$. We want to show that no sequence of irreducible solutions can converge to a reducible solution. We can apply the same perturbative argument used in [13], based on the local Kuranishi model. In fact if $(A, \psi)$ is an irreducible solution which is sufficiently close to a reducible solution $\left(A_{0}+\nu, 0\right)$, then we have an expansion

$$
A=A_{0}+\nu+\epsilon \alpha_{1}+\epsilon^{2} \alpha_{2}+\cdots,
$$

and

$$
\psi=\epsilon \psi_{1}+\epsilon^{2} \psi_{2}+\epsilon^{3} \psi_{3}+\cdots .
$$

Using the equation (15) and the condition that $\operatorname{Ker}\left(\partial_{A_{0}+\nu}\right)=0$ we get that $\psi_{i} \equiv 0$ for all $i$, in contradiction with the assumption that $(A, \psi)$ is an irreducible solution. Thus, for a fixed choice of the Spin ${ }^{c}$-structure, the framed moduli space $\mathcal{M}^{0}$ consists of finitely many circles and a unique point fixed by the $U(1)$ action.

Corollary 3.5. Under the choice of a generic perturbation (and of a generic metric in the case with $b^{1}(Y)=0$ ), the functional $\mathcal{C}_{\rho}$ satisfies the Morse-Bott property.

Remark 3.6. The condition that $\partial_{A}$ satisfies $\operatorname{Ker}\left(\partial_{A}\right)=0$ at the reducible critical point breaks the space of metrics and perturbations into chambers, as discussed in Theorem 2.8. The Floer groups can be expected to change when crossing a wall corresponding to metrics with non-trivial $\operatorname{Ker}\left(\partial_{A}\right)$. The problem of possible dependence of the metric in this case was already addressed by Donaldson in [15]. In Section 7, we prove that indeed the non-equivariant Floer groups do change when crossing a wall. In fact, we derive explicit wall crossing formulae for the Euler characteristic of the Floer homology.

\subsection{Gradient flow lines.}

Denote by $O_{a}$ the critical orbit in $\mathcal{M}^{0}$ that corresponds to a critical point $a \in \mathcal{M}$. From Proposition 3.4 we know that $\mathcal{M}^{0}$ is a $U(1)$-fibration over $\mathcal{M}$, where $O_{a}$ is a circle if $a$ is irreducible and $O_{a}$ is the point $a$ itself otherwise. 
Let us introduce the configuration space $\mathcal{A}_{k, \delta}\left(O_{a}, O_{b}\right)$. Consider the space $\mathcal{A}_{k, \delta}\left(\left(A_{0}, \psi_{0}\right),\left(A_{1}, \psi_{1}\right)\right)$ of elements $(\mathbb{A}, \Psi)$ of the form

$$
(\mathbb{A}, \Psi) \in(A(t), \psi(t))+L_{k, \delta}^{2}\left(\Omega^{1}(Y \times \mathbb{R}) \oplus \Gamma\left(S^{+} \otimes L\right)\right),
$$

where $(A(t), \psi(t))$ is a smooth path such that $(A(t), \psi(t)) \equiv\left(A_{0}, \psi_{0}\right)$ for $t \leq$ 0 and $(A(t), \psi(t)) \equiv\left(A_{1}, \psi_{1}\right)$ for $t \geq 1$, with $\mathcal{G}^{0}$-gauge classes $\left[A_{0}, \psi_{0}\right] \in O_{a}$ and $\left[A_{1}, \psi_{1}\right] \in O_{b}$.

The space $\mathcal{A}_{k, \delta}\left(O_{a}, O_{b}\right)$ is given by

$$
\mathcal{A}_{k, \delta}\left(O_{a}, O_{b}\right)=\bigcup_{\lambda \in U(1)} \mathcal{A}_{k, \delta}\left(\lambda\left(A_{0}, \psi_{0}\right), \lambda\left(A_{1}, \psi_{1}\right)\right)
$$

The space is endowed with a $U(1)$-action.

Let $\mathcal{B}_{k \delta}^{0}\left(O_{a}, O_{b}\right)$ be the quotient of $\mathcal{A}_{k, \delta}\left(O_{a}, O_{b}\right)$ modulo the free action of the gauge group $\mathcal{G}_{k+1, \delta}^{0}\left(O_{a}, O_{b}\right)$ of based gauge transformations, modeled on the $L_{k+1, \delta}^{2}$ completion of the Lie algebra, that decay, as $t \rightarrow \pm \infty$, to elements $\lambda_{ \pm \infty}$ in the stabilizers $G_{a}$ and $G_{b}$. The quotient has an induced $U(1)$-action and endpoint maps

$$
e_{a}^{+}: \mathcal{B}_{k \delta}^{0}\left(O_{a}, O_{b}\right) \rightarrow O_{a}
$$

and

$$
e_{b}^{-}: \mathcal{B}_{k \delta}^{0}\left(O_{a}, O_{b}\right) \rightarrow O_{b}
$$

We denote by $\mathcal{M}\left(O_{a}, O_{b}\right)$ the moduli space of solutions of equations (20) and (21) in $\mathcal{B}_{k \delta}^{0}\left(O_{a}, O_{b}\right)$. These will be our moduli spaces of flow lines connecting the orbits $O_{a}$ and $O_{b}$.

Remark 3.7. Suppose one of $O_{a}$ and $O_{b}$ is not the fixed point $\theta$. Then no reducible solution arises among the flow lines $\mathcal{M}\left(O_{a}, O_{b}\right)$.

In fact, either $\left(A_{a}, \psi_{a}\right)$ or $\left(A_{b}, \psi_{b}\right)$ has non-trivial spinor. Thus $\psi(t)$ has to be non-trivial, in fact, the exponential weight on the Sobolev norms forces the elements in (26) to decay to the endpoints, at least one of which has non-vanishing spinor. Thus, the space $\mathcal{A}_{k, \delta}\left(O_{a}, O_{b}\right)$ only contains irreducible lines. The action of $\mathcal{G}_{k+1, \delta}^{0}\left(O_{a}, O_{b}\right)$ is free and $\mathcal{B}_{k, \delta}^{0}\left(O_{a}, O_{b}\right)$ is a manifold with a free $U(1)$-action. 


\subsection{Relative Morse Index.}

We can rephrase Proposition 2.12 in the case of framed moduli spaces as follows.

Theorem 3.8. Suppose $O_{a}$ and $O_{b}$ are critical orbits of $\mathcal{C}_{\rho}$ in $\mathcal{B}^{0}$. If $Y$ is a rational homology sphere, assume that the metric and perturbation are chosen generically so that $\mathcal{C}_{\rho}$ satisfies the Morse-Bott condition. Let $\left\{\lambda_{a}\right\}$ and $\left\{\lambda_{b}\right\}$ be the eigenvalues of the Hessian operators $Q_{a}, Q_{b}$ (that is the Hessian in $\mathcal{B}^{0}$ restricted to the directions orthogonal to the $U(1)$-orbits). Choose a weight $\delta$ satisfying $0<\delta<\min \left\{\left|\lambda_{a}\right|,\left|\lambda_{b}\right|\right\}$. Then the linearization $\mathcal{L}_{(\mathbb{A}, \Psi)}$ at a solution $[\mathbb{A}, \Psi] \in \mathcal{B}_{k, \delta}^{0}\left(O_{a}, O_{b}\right)$ of $(20)$ and (21) is a Fredholm operator from $L_{k, \delta}^{2}$ to $L_{k-1, \delta}^{2}$. The virtual dimension of the moduli space $\mathcal{M}\left(O_{a}, O_{b}\right)$ is given by the index of $\mathcal{L}_{(\mathbb{A}, \Psi)}$ and is obtained as

$$
\operatorname{dim} \mathcal{M}\left(O_{a}, O_{b}\right)=\sigma\left(O_{a}, O_{b}\right)+1-\operatorname{dim} G_{a},
$$

where $\sigma\left(O_{a}, O_{b}\right)$ is the spectral flow of the operator $\nabla \mathcal{F}$ on a path $(A(t), \psi(t))$ in $\mathcal{A}$ corresponding to $(\mathbb{A}, \Psi)$. The quantity $\sigma\left(O_{a}, O_{b}\right)$ is independent of the path in $\mathcal{B}_{k}^{0}$; by additivity of the spectral flow, it can be written as

$$
\sigma\left(O_{a}, O_{b}\right)=\mu\left(O_{a}\right)-\mu\left(O_{b}\right),
$$

where $\mu\left(O_{a}\right)$ is the flow of $\nabla \mathcal{F}$ on a path connecting the orbit $O_{a}$ to a fixed orbit in $\mathcal{M}^{0}$.

Proof. The fact that the linearization $\mathcal{L}_{(\mathbb{A}, \Psi)}$ on the spaces $\mathcal{B}_{k, \delta}(a, b)$ and on $\mathcal{B}_{k, \delta}\left(O_{a}, O_{b}\right)$ is Fredholm follows from Theorem 6.2 of [36], or Lemma 8.3.1 of [47] (cf. the previous discussion of this point in Proposition 2.15), provided that the operator $T$ has trivial kernel at the points $a$ and $b$ and the weight $\delta$ is smaller than the least eigenvalue of $T$, see also [61].

We shall write $\operatorname{Ind}_{e}\left(\mathcal{L}_{(\mathbb{A}, \Psi)}\right)$ for the index formula in $\mathcal{B}_{k \delta}^{0}\left(O_{a}, O_{b}\right)$. The subscript denotes the fact that we are computing

$$
\operatorname{Ind}_{e}\left(\mathcal{L}_{(\mathbb{A}, \Psi)}\right)=\operatorname{dim} \operatorname{Ker}_{e}\left(\mathcal{L}_{(\mathbb{A}, \Psi)}\right)-\operatorname{dim} \operatorname{Coker}\left(\mathcal{L}_{(\mathbb{A}, \Psi)}\right),
$$

where $\operatorname{Ker}_{e}\left(\mathcal{L}_{(\mathbb{A}, \Psi)}\right)$ is the extended kernel, which consists of solutions $(\alpha, \Phi)$ of $\mathcal{L}_{(\mathbb{A}, \Psi)}(\alpha, \Phi)=0$, with $(\alpha, \Phi)-\left(\alpha_{-}, \phi_{-}\right)$in $L_{k, \delta}^{2}$, where $\left(\alpha_{-}, \phi_{-}\right)$is a solution of $L_{A_{a}, \psi_{a}}\left(\alpha_{-}, \phi_{-}\right)=0$, namely a tangent vector to the orbit $O_{a}$ in $\mathcal{T B}^{0}$. Notice that this $\operatorname{Ker}_{e}\left(\mathcal{L}_{(\mathbb{A}, \Psi)}\right)$ is the correct space representing the tangent space of $\mathcal{M}\left(O_{a}, O_{b}\right)$ in $\mathcal{B}_{k \delta}^{0}\left(O_{a}, O_{b}\right)$. The cokernel is simply given by 
$L_{k-1, \delta}^{2}$ solutions of $\mathcal{L}_{(\mathbb{A}, \Psi)}^{*}(f, \beta, \xi)=0$, as in the setting of [3] I, p. 58. The formula for the index of $\mathcal{L}_{(\mathbb{A}, \Psi)}$ on $\mathcal{B}_{k, \delta}^{0}\left(O_{a}, O_{b}\right)$

$$
\operatorname{Ind}_{e}\left(\mathcal{L}_{(\mathbb{A}, \Psi)}\right)=\sigma\left(O_{a}, O_{b}\right)+1-\operatorname{dim} G_{a},
$$

then follows from the splitting of the index that will be proven in Corollary 4.18, together with the additivity of the spectral flow, and [3] III pg.95.

Thus we obtain the virtual dimension of $\mathcal{M}\left(O_{a}, O_{b}\right)$,

$$
\operatorname{dim} \mathcal{M}\left(O_{a}, O_{b}\right)=\sigma\left(O_{a}, O_{b}\right)+1-\operatorname{dim} G_{a},
$$

where the +1 contribution depends upon the presence of the $U(1)$-action and $\operatorname{dim} G_{a}$ is the dimension of the stabilizer of the point $a$.

The relative Morse index is well defined. To see this, we have to examine the spectral flow of the operator $\nabla \mathcal{F}_{(A(t), \psi(t))}$ on a path $(A(t), \psi(t))$ in $\mathcal{A}$ with endpoints $(A, \psi)$ and $\lambda(A, \psi)$, where $\lambda$ is a gauge transformation satisfying

$$
\frac{i}{2 \pi} \int_{Y} c_{1}(L) \wedge \lambda^{-1} d \lambda=0
$$

According to [3] III p. 95, and [64], this spectral flow is just the index of $\mathcal{L}$ on the manifold $Y \times S^{1}$. The index on a closed four-manifold is

$$
\operatorname{Ind}(\mathcal{L})=c_{1}(L)^{2}-\frac{2 \chi+3 \sigma}{4}
$$

(cf. Corollary 4.6.2 of [45], or Theorem 2.3.8 of [39]), and this quantity vanishes in the case of a manifold of the form $Y \times S^{1}$.

Given the relative Morse index, we can define the Morse index of a critical orbit up to fixing arbitrarily the index of a particular solution. In the case with $b^{1}(Y)>0$ there is no canonical choice, hence the grading of the Floer complex is only defined up to an integer. When $b^{1}(Y)=0$ we can remove this ambiguity by fixing the trivial solution $\theta=\left[A_{0}+\nu, 0\right]$ to have index zero.

Notice that, in the case with $b^{1}(Y)>0, c_{1}(L) \neq 0$, and $[* \rho]=0$, the relative Morse index would be defined only up to a periodicity if we considered solutions modulo the full gauge group $\tilde{\mathcal{G}}$. This is related to the fact that the functional $\mathcal{C}$ is well defined on $\mathcal{A} / \mathcal{G}$, but is only defined as a circle valued functional on $\mathcal{A} / \tilde{\mathcal{G}}$. The relation between $\mathbb{Z}$-graded and $\mathbb{Z}_{l^{-}}$ graded Seiberg-Witten Floer theory will be sketched briefly in Section 4.1, in Remark 4.5. 


\subsection{Decay estimate.}

In this subsection we introduce some analytic properties of the functional $\mathcal{C}_{\rho}$. We show that finite energy solutions of the flow equations necessarily converge to asymptotic values that lie on some critical orbit. Moreover, we show that, if $O_{a}$ or $O_{b}$ is an irreducible orbit, then the flow lines in the moduli space $\mathcal{M}\left(O_{a}, O_{b}\right)$ decay exponentially towards the endpoints. Notice that in this case the moduli space $\mathcal{M}\left(O_{a}, O_{b}\right)$ only contains irreducible flow lines.

We give the following preliminary definition.

Definition 3.9. A smooth path $(A(t), \psi(t))$ in $\mathcal{A}$ is of finite energy if the integral

$$
\int_{-\infty}^{\infty}\left\|\nabla \mathcal{C}_{\rho}(A(t), \psi(t))\right\|_{L^{2}}^{2} d t<\infty
$$

is finite.

Notice that any solution of (18) and (19) with asymptotic values in $O_{a}$ and $O_{b}$ is of finite energy, in fact in this case the total variation of the functional $\mathcal{C}_{\rho}$ along the path $(A(t), \psi(t))$ is finite and (28) satisfies

$$
\int_{-\infty}^{\infty}\left\|\nabla \mathcal{C}_{\rho}(A(t), \psi(t))\right\|_{L^{2}}^{2} d t \leq C\left(\mathcal{C}_{\rho}(a)-\mathcal{C}_{\rho}(b)\right)
$$

because of the assumptions on the perturbation $q_{(\mathbb{A}, \Psi)}$. Finite energy solutions of the flow equations have nice properties: they necessarily decay to asymptotic values that are critical points of $\mathcal{C}_{\rho}$ as we prove in Corollary 3.11 and in Theorem 3.12. We begin by introducing some analytic properties of the functional $\mathcal{C}_{\rho}$ (see also [25], [48], [13]).

Lemma 3.10. Let $\mathcal{M}_{\rho}$ be the moduli space of critical points of $\mathcal{C}_{\rho}$, with $\rho$ a sufficiently small perturbation. For any $\epsilon>0$ there is a $\lambda>0$ such that, if the $L_{1}^{2}$-distance of a point $[A, \psi]$ of $\mathcal{B}$ to all the points in $\mathcal{M}_{\rho}$ is at least $\epsilon$, then

$$
\left\|\nabla \mathcal{C}_{\rho}(A, \psi)\right\|_{L^{2}}>\lambda \text {. }
$$

Proof. For a sequence $\left[A_{i}, \psi_{i}\right]$ of elements of $\mathcal{B}$ with a distance at least $\epsilon$ from all the critical points, such that

$$
\left\|\nabla \mathcal{C}_{\rho}\left(A_{i}, \psi_{i}\right)\right\|_{L^{2}} \rightarrow 0,
$$


as $i \rightarrow \infty$, we would have

$$
\left\|* F_{A_{i}}-\sigma\left(\psi_{i}, \psi_{i}\right)-i \rho\right\|+\left\|\partial_{A_{i}} \psi_{i}\right\| \rightarrow 0
$$

Thus, there is a constant $C$ such that

$$
\int_{Y}\left|* F_{A_{i}}-\sigma\left(\psi_{i}, \psi_{i}\right)-i \rho\right|^{2}+\left|\partial_{A_{i}} \psi_{i}\right|^{2} d v<C
$$

If the perturbation $\rho$ is sufficiently small, the Weizenböck formula implies that

$$
\int_{Y}\left|F_{A_{i}}\right|^{2}+\left|\sigma\left(\psi_{i}, \psi_{i}\right)\right|^{2}+\frac{\kappa}{2}\left|\psi_{i}\right|^{2}+2\left|\nabla_{A_{i}} \psi_{i}\right|^{2} d v<C .
$$

Thus we have a uniform bound on the norms $\left\|\psi_{i}\right\|_{L^{4}},\left\|F_{A_{i}}\right\|_{L^{2}}$, and $\left\|\nabla_{A_{i}} \psi_{i}\right\|_{L^{2}}$. An elliptic estimate shows that there is a subsequence that converges in the $L_{1}^{2}$ norm to a solution of the critical point equations (15), and this contradicts the assumption.

Corollary 3.11. Let $(A(t), \psi(t))$ be a smooth finite energy solution of equations (18) and (19) with a smooth perturbation $q$. Then there exist critical points $a$ and $b$ of $\mathcal{C}_{\rho}$, such that the $\lim _{t \rightarrow \pm \infty}(A(t), \psi(t))$ are in the gauge classes of $a$ and $b$.

Proof. The finite energy condition (28) implies that

$$
\left\|\nabla \mathcal{C}_{\rho}(A(t), \psi(t))\right\| \rightarrow 0
$$

as $t \rightarrow \pm \infty$. The Palais-Smale condition of Lemma 3.10 implies that there exist $T$ large, such that for $|t|>T,[A(t), \psi(t)]$ lies in a very small $\epsilon$ neighborhood of critical points of $\mathcal{C}_{\rho}$. The claim at the level of representatives $(A(t), \psi(t))$ follows from the finite energy condition and the flow equations.

Now we prove the exponential decay property.

Theorem 3.12. Let $O_{a}$ and $O_{b}$ be non-degenerate critical orbits in $\mathcal{B}_{0}$. There exists a weight $\delta>0$ such that the following holds. Suppose given any solution $[\mathbb{A}, \Psi]$ of $(20)$ and (21) that is represented by a smooth pair $(A(t), \psi(t))$ in a temporal gauge, with asymptotic values $\left(A_{a}, \psi_{a}\right)$ and 
$\left(A_{b}, \psi_{b}\right)$ in $O_{a}$ and $O_{b}$. Then there exists a constant $K$ such that, for $t$ outside an interval $[-T, T]$, the distance in any fixed $\mathcal{C}^{l}$-topology of $(A(t), \psi(t))$ from the endpoints is

$$
\operatorname{dist}_{C^{l}}\left((A(t), \psi(t)),\left(A_{i}, \psi_{i}\right)\right)<K \exp (-\delta|t|),
$$

with $i=a$ if $t<-T$ and $i=b$ if $t>T$.

Proof. The proof consists of a few steps. Let us consider the decaying as $t \rightarrow \infty$; the other case with $t \rightarrow-\infty$ is analogous.

For simplicity of notation we shall prove the Theorem in the case of flow lines in $\mathcal{B}$, with the action of the gauge group $\mathcal{G}$ of gauge maps that satisfy (2), and perturbation $\rho$ satisfying $[* \rho]=0$ in cohomology. This ensures that the functional $\mathcal{C}_{\rho}$ is $\mathbb{R}$-valued. All the claims and the proofs extend directly to the case of the based space with the $\mathcal{G}^{0}$-action and a non-degenerate critical orbit $O_{b}$ in the quotient space $\mathcal{B}_{0}$. In this case, the distance of $(A, \Psi)$ from the orbit $O_{b}$ is the minimal distance from points on the orbit.

Lemma 3.13. Suppose $b$ is a non-degenerate critical point of $\mathcal{C}_{\rho}$. Then there exists a constant $C_{b}$ such that if the $L^{2}$-distance from $[A, \psi]$ to $b$ is sufficiently small, then we have the following estimate of the $L^{2}$-distance from $[A, \psi]$ to $b$ :

$$
\operatorname{dist}_{L^{2}}([A, \psi], b) \leq C_{b}\left\|\nabla \mathcal{C}_{\rho}(A, \psi)\right\|_{L^{2}} .
$$

Proof. Consider the Hessian operator $T_{b}$ acting as an unbounded operator on the space of $L^{2}$ connections and sections. Since $b$ is a non-degenerate critical point, we have that

$$
h_{b}=\max \left\{\frac{1}{\left|\lambda_{i}\right|} \mid \lambda_{i} \text { is a eigenvalue of the Hessian operator } T_{b} \text { at } b\right\}
$$

exists and is bounded. We know that $[A, \psi] \mapsto \nabla \mathcal{C}_{\rho}(A, \psi)$ defines a $L^{2}$ tangent section, which is smooth and transverse to zero at $b$. Thus, for any $\epsilon>0$, we may choose a small neighborhood $U_{b}$ of $b$ which may be identified with a small neighborhood of 0 in the $L^{2}$-tangent space of $\mathcal{B}$ at $b$, such that for all $(A, \psi)=b+(\alpha, \phi)$ in $U_{b}$ we have

$$
\left\|\nabla \mathcal{C}_{\rho}(A, \psi)-T_{b}(\alpha, \phi)\right\|_{L^{2}} \leq \epsilon
$$


where we write $\nabla \mathcal{C}_{\rho}(A, \psi)$ as the sum of the linear and a non-linear term,

$$
\nabla \mathcal{C}_{\rho}(A, \psi)=T_{b}(\alpha, \phi)+N(\alpha, \phi),
$$

with $N(\alpha, \phi)=(\sigma(\phi, \phi), \alpha \cdot \phi)$.

When the neighborhood $U_{b}$ is small enough, we can ensure that

$$
\|N(\alpha, \phi)\| \leq 1 / 2\left\|\nabla \mathcal{C}_{\rho}(A, \psi)\right\|,
$$

so that we have

$$
\begin{aligned}
\left\|T_{b}(\alpha, \phi)\right\| & \leq\left\|\nabla \mathcal{C}_{\rho}(A, \psi)\right\|+\|N(\alpha, \phi)\| \\
& \leq 3 / 2\left\|\nabla \mathcal{C}_{\rho}(A, \psi)\right\| .
\end{aligned}
$$

Thus, we get the following estimate:

$$
\begin{aligned}
& \operatorname{dist}_{L^{2}}([A, \psi], b) \\
& =\|(\alpha, \phi)\|_{L^{2}} \\
& \leq h_{b}\left\|\left(T_{b}(\alpha, \phi)\right)\right\|_{L^{2}} \\
& \leq \frac{3}{2} h_{b}\left\|\left(\nabla \mathcal{C}_{\rho}([A, \psi])\right)\right\|_{L^{2}(Y)} \\
& \leq \frac{3}{2} h_{b}\left\|\nabla \mathcal{C}_{\rho}([A, \psi])\right\|_{L^{2}} .
\end{aligned}
$$

The Lemma follows upon choosing the constant $C_{b}$ with

$$
C_{b}>\frac{3}{2} h_{b}
$$

Claim 1. Let $(A(t), \psi(t))$ be a representative of a path in $\mathcal{B}$, such that

$$
\lim _{t \rightarrow \infty}(A(t), \psi(t))=\left(A_{b}, \psi_{b}\right)
$$

where $b=\left[A_{b}, \psi_{b}\right]$ is a non-degenerate critical point in $\mathcal{M}_{\rho}$. Then there is a $T_{0} \gg 0$ and a constant $K_{b}$, such that the inequality

$$
\left|\mathcal{C}_{\rho}(A(t), \psi(t))-\mathcal{C}_{\rho}\left(A_{b}, \psi_{b}\right)\right| \leq K_{b}\left\|\nabla \mathcal{C}_{\rho}(A(t), \psi(t))\right\|_{L^{2}}^{2}
$$

holds for all $t \geq T_{0}$.

Proof of Claim 1. Choose $T_{0}$ such that for all $t \geq T_{0}$ the path $(A(t), \psi(t))$ lies in a neighborhood $U_{b}$ of $b$ for which the result of Lemma 3.13 holds. Notice that such a $T_{0}$ depends in general on which path $(A(t), \psi(t))$ is considered. 
With the notation of (4) and (13), we have

$$
\mathcal{C}_{\rho}(A, \psi) \sim \mathcal{C}_{\rho}\left(A_{b}, \psi_{b}\right)+\mathcal{F}_{b}(\alpha, \phi)+\nabla \mathcal{F}_{b}(\alpha, \phi)
$$

where $(A, \psi)=b+(\alpha, \phi)$ in $U_{b}$. Since $b$ is a critical point, we have $\mathcal{F}_{b} \equiv 0$.

Thus we get

$$
\left|\mathcal{C}_{\rho}(A, \psi)-\mathcal{C}_{\rho}\left(A_{b}, \psi_{b}\right)\right| \leq\left|\left\langle T_{b}(\alpha, \phi),(\alpha, \phi)\right\rangle\right|
$$

Now applying Lemma 3.13 we obtain the estimate

$$
\begin{aligned}
\left|\mathcal{C}_{\rho}(A, \psi)-\mathcal{C}_{\rho}\left(A_{b}, \psi_{b}\right)\right| & \leq\left\|T_{b}(\alpha, \phi)\right\| \cdot\|(\alpha, \phi)\| \\
& \leq \frac{3}{2} C_{b}\left\|\nabla \mathcal{C}_{\rho}(A, \psi)\right\|_{L^{2}}^{2}
\end{aligned}
$$

This completes the proof of Claim 1 , with the constant $K_{b} \geq 3 C_{b} / 2$.

Claim 2. For a finite energy solution $(A(t), \psi(t))$ of $(18)$ and (19), the inequality

$$
\begin{aligned}
\frac{1}{2} \int_{t}^{\infty}\left\|\nabla \mathcal{C}_{\rho}(A(s), \psi(s))\right\|_{L^{2}}^{2} d s & \leq \mathcal{C}_{\rho}(A(t), \psi(t))-\mathcal{C}_{\rho}\left(A_{b}, \psi_{b}\right) \\
& \leq \frac{3}{2} \int_{t}^{\infty}\left\|\nabla \mathcal{C}_{\rho}(A(s), \psi(s))\right\|_{L^{2}}^{2} d s
\end{aligned}
$$

holds for large $t$.

Proof of Claim 2. Without loss of generality we can assume that the perturbation in $\mathcal{P}$ satisfies Condition (4) of 2.10 with $C_{0}<1 / 2$, so that

$$
\left\|q_{(\mathbb{A}, \Psi)}(t)\right\|_{L^{2}}<\frac{1}{2}\left\|\nabla \mathcal{C}_{\rho}(A(t), \psi(t))\right\|_{L^{2}}
$$

Thus, we can replace the equality

$$
\begin{aligned}
& \mathcal{C}_{\rho}(A(t), \psi(t))-\mathcal{C}_{\rho}\left(\left(A_{b}, \psi_{b}\right)\right) \\
& =\int_{t}^{\infty}-\frac{d}{d s} \mathcal{C}_{\rho}(A(s), \psi(s)) d s \\
& =\int_{t}^{\infty}-\left\langle\frac{d}{d s}(A(s), \psi(s)), \nabla \mathcal{C}_{\rho}(A(s), \psi(s))\right\rangle d s \\
& =\int_{t}^{\infty}\left\|\nabla \mathcal{C}_{\rho}(A(s), \psi(s))\right\|^{2} d s,
\end{aligned}
$$

that holds for solutions of the unperturbed equations with the inequality of Claim 2 for solutions of the perturbed equations. 
Claim 3. Let $(A(t)), \psi(t))$ be a finite energy solution of the equations (18) and (19). The quantity

$$
E(t)=\int_{t}^{\infty}\left\|\nabla \mathcal{C}_{\rho}(A(s), \psi(s))\right\|_{L^{2}}^{2} d s
$$

decays exponentially as $t \rightarrow \infty$.

Proof of Claim 3. In fact, the inequality of Claim 2 gives the first inequality in the following estimate:

$$
\begin{aligned}
E(t) & \leq 2\left(\mathcal{C}_{\rho}(A(t), \psi(t))-\mathcal{C}_{\rho}\left(A_{b}, \psi_{b}\right)\right) \\
& \leq K_{b}\left\|\nabla \mathcal{C}_{\rho}(A(t), \psi(t))\right\|^{2} \\
& =-K_{b} \frac{d}{d t} E(t) .
\end{aligned}
$$

The second inequality follows from Claim 1.

Claim 4. For large $t$ we have the inequality

$$
\operatorname{dist}_{L^{2}}\left((A(t), \psi(t)),\left(A_{b}, \psi_{b}\right)\right) \leq K\left(\int_{t-1}^{\infty}\left\|\nabla \mathcal{C}_{\rho}(A(s), \psi(s))\right\|_{L^{2}}^{2} d s\right)^{1 / 2}
$$

when $x(t)=(A(t), \psi(t))$ is a finite energy solution of (18) and (19).

Proof of Claim 4. We can prove that the following inequality holds true for $t \geq T_{0}$ :

$$
\int_{t}^{\infty}\left\|\frac{d}{d s} x(s)\right\|_{L^{2}} d s \leq \frac{2 K_{b}}{1-C_{1}}\left(\mathcal{C}_{\rho}(x(t))-\mathcal{C}_{\rho}(b)\right)^{1 / 2},
$$

where $T_{0}$ is such that for all $t \geq T_{0}$ the perturbation $q_{(\mathbb{A}, \Psi)}$ satisfies the inequality

$$
\left\|q_{(A(t), \psi(t))}(t)\right\|_{L^{2}} \leq C_{1}\left\|\nabla \mathcal{C}_{\rho}(A(t), \psi(t))\right\|_{L^{2}},
$$

with $0<C_{1}<1$, as in property (4) of definition 2.10. Moreover, $T_{0}$ is such that Lemma 3.13 holds. The proof of this inequality follows [58] Lemma 3.1, p. 542. We have

$$
\begin{aligned}
& \left\|\frac{d}{d t} x(t)\right\|^{2}+\left\|\nabla \mathcal{C}_{\rho}(x(t))\right\|^{2}+2\left\langle\frac{d}{d t} x(t), \nabla \mathcal{C}_{\rho}(x(t))\right\rangle \\
& =\|q(t)\|^{2} \leq C_{1}^{2}\left\|\frac{d}{d t} x(t)\right\|^{2} \leq C_{1}\left\|\frac{d}{d t} x(t)\right\|^{2} .
\end{aligned}
$$


Thus we get

$$
\begin{aligned}
-\left\langle\frac{d}{d t} x(t), \nabla \mathcal{C}_{\rho}(x(t))\right\rangle & \geq \frac{\left(1-C_{1}\right)}{2}\left(\left\|\frac{d}{d t} x(t)\right\|^{2}+\left\|\nabla \mathcal{C}_{\rho}(x(t))\right\|^{2}\right) \\
& \geq\left(1-C_{1}\right)\left\|\frac{d}{d t} x(t)\right\| \cdot\left\|\nabla \mathcal{C}_{\rho}(x(t))\right\|,
\end{aligned}
$$

for all $t \in\left[T_{0}, \infty\right)$. That is,

$$
-\frac{d}{d t} \mathcal{C}_{\rho}(x(t)) \geq\left(1-C_{1}\right)\left\|\frac{d}{d t} x(t)\right\| \cdot\left\|\nabla \mathcal{C}_{\rho}(x(t))\right\| .
$$

We obtain the inequality

$$
\begin{aligned}
& -\frac{d}{d t}\left(\mathcal{C}_{\rho}(x(t))-\mathcal{C}_{\rho}(b)\right)^{1 / 2} \\
& \geq \frac{\left(1-C_{1}\right)}{2}\left(\mathcal{C}_{\rho}(x(t))-\mathcal{C}_{\rho}(b)\right)^{-1 / 2}\left\|\frac{d}{d t} x(t)\right\| \cdot\left\|\nabla \mathcal{C}_{\rho}(x(t))\right\| \\
& \geq \frac{\left(1-C_{1}\right)}{2 K_{b}}\left\|\frac{d}{d t} x(t)\right\| .
\end{aligned}
$$

The last inequality follows from Claim 1 . We can now integrate both sides to obtain

$$
\begin{aligned}
\int_{t}^{\infty}\left\|\frac{d}{d s} x(s)\right\|_{L^{2}} d s & \leq \frac{2 K_{b}}{\left(1-C_{1}\right)} \int_{t}^{\infty}-\frac{d}{d s}\left(\mathcal{C}_{\rho}(x(s))-\mathcal{C}_{\rho}(b)\right)^{1 / 2} d s \\
& =\frac{2 K_{b}}{\left(1-C_{1}\right)}\left(\mathcal{C}_{\rho}(x(t))-\mathcal{C}_{\rho}(b)\right)^{1 / 2}
\end{aligned}
$$

Thus by Claim 2 we have

$$
\operatorname{dist}_{L^{2}}\left((A(t), \psi(t)),\left(A_{b}, \psi_{b}\right)\right) \leq K\left(\int_{t}^{\infty}\left\|\nabla \mathcal{C}_{\rho}(A(s), \psi(s))\right\|_{L^{2}}^{2} d s\right)^{1 / 2} .
$$

The exponential decay of $E(t)$ proves the claim of the Theorem for the case of $L^{2}$-topology. Smooth estimates then follow by a bootstrapping argument and elliptic regularity. This completes the proof of the Theorem.

Analogous exponential decay estimates have been proven in [48], cf. [4] and [13]. 


\subsection{Transversality of $\mathcal{M}\left(O_{a}, O_{b}\right)$.}

We have the following transversality result for the moduli spaces $\mathcal{M}\left(O_{a}, O_{b}\right)$.

Proposition 3.14. Suppose given two orbits $O_{a}$ and $O_{b}$ in $\mathcal{M}^{0}$. For a generic choice of the perturbation $P \in \mathcal{P}$, the space $\mathcal{M}\left(O_{a}, O_{b}\right)$ is a smooth $\mathbb{R} \times U(1)$-manifold with dimension given by $\mu\left(O_{a}\right)-\mu\left(O_{b}\right)+1-\operatorname{dim} G_{a}$. $\mathcal{M}\left(O_{a}, O_{b}\right)$ is non-empty only if $\mu\left(O_{a}\right)-\mu\left(O_{b}\right) \geq 1$. There are endpoint maps

$$
e_{a}^{+}: \mathcal{M}\left(O_{a}, O_{b}\right) \rightarrow O_{a}
$$

and

$$
e_{b}^{-}: \mathcal{M}\left(O_{a}, O_{b}\right) \rightarrow O_{b}
$$

that are smooth $U(1)$-equivariant maps.

The computation of the virtual dimension follows from Theorem 3.8. The transversality statement follows from Proposition 2.14, together with the Remark 3.7, to the effect that the smooth manifolds $\mathcal{M}(a, b)$ of Proposition 2.14 are just the quotient of $\mathcal{M}\left(O_{a}, O_{b}\right)$ with respect to the free $U(1)$ action. The properties of the endpoint maps follow from the discussion on the convergence to the endpoints of Section 3.4.

We have the analogue of Corollary 2.17 in this case.

Corollary 3.15. Suppose $\theta$ is the reducible critical point in $\mathcal{M}$ and $b$ any point in $\mathcal{M}$. Then, after a generic perturbation, $\mathcal{M}\left(\theta, O_{b}\right)$ is a smooth $\mathbb{R} \times$ $U(1)$-manifold with dimension $\mu(\theta)-\mu\left(O_{b}\right)$. Moreover, $\mathcal{M}\left(\theta, O_{b}\right)$ is nonempty only if $\mu(\theta)-\mu\left(O_{b}\right) \geq 2$.

\section{Boundary structure.}

The purpose of this part of the work is to study the compactification of the moduli spaces $\hat{\mathcal{M}}\left(O_{a}, O_{b}\right)$. The first step consists of showing the existence of a compactification, obtained by adding a certain set of broken trajectories, namely trajectories that break through other critical orbits of intermediate relative Morse index. We shall introduce the notation $\hat{\mathcal{M}}\left(O_{a}, O_{b}\right)^{*}$ for the compactified moduli spaces. Proving the existence of the compactification by broken trajectories is dealt with in Section 4.1. Section 4.2 then deals with the gluing construction, and Section 4.3 analyzes the fine structure of the compactification.

It is perhaps useful to recall why in Floer theory it is necessary to develop the full gluing construction, as in Section 4.2. The results of Section 4.1 are 
sufficient to prove the existence of a compactification for the moduli spaces of flow lines, obtained by adding broken trajectories. The points of the ideal boundary are not completely identified, and the purpose of the gluing construction of Section 4.2 is precisely to identify all the broken trajectories that appear in the boundary. Recall also that, in the Floer complex, the boundary operator is obtained by counting elements in the oriented moduli spaces $\hat{\mathcal{M}}(a, b)^{*}$ for critical points $a, b$ of relative index one. The statement that this is a boundary operator, namely that $\partial \circ \partial=0$, depends precisely upon having a complete description of the ideal boundary in the compactified moduli spaces $\hat{\mathcal{M}}(a, c)^{*}$ for critical points $a$ and $c$ of relative index two. In other words, proving that all the broken trajectories

$$
\cup_{\mu(a)>\mu(b)>\mu(c)} \hat{\mathcal{M}}(a, b)^{*} \times \hat{\mathcal{M}}(b, c)^{*}
$$

appear in the compactification $\hat{\mathcal{M}}(a, c)^{*}$ is necessary in order to have a chain complex. In the equivariant setting we are considering here, there are components of the boundary operator $D$ which are obtained by pullback and push-forward of forms on compactified moduli spaces $\hat{\mathcal{M}}\left(O_{a}, O_{b}\right)^{*}$, for orbits of relative index one, and on $\hat{\mathcal{M}}\left(O_{a}, O_{c}\right)^{*}$ for orbits of relative index two (see the explicit description of the boundary operator given in Section 5 , in (72). The proof that $D \circ D=0$ relies upon a version of Stokes' theorem for manifolds with corners (as in [7]). For this reason, it is necessary to show that the compactification of the moduli spaces of flow lines of Section 4.1 has the structure of a smooth manifold with corners. This is proved in the case of the codimension one boundary in Section 4.2, and the generalization to the strata of higher codimension is considered in Section 4.3.

Thus, in Floer theory, a very elaborate analysis of the compactification of the moduli spaces of flow lines is required, in order to construct the Floer complex. This situation is essentially different from other issues of compactification in gauge theory. For instance, in the case of Donaldson invariants [18], one only needs to show the existence of the Uhlenbeck compactification of the moduli space of anti-self-dual connections. The definition of the invariants and the proof of the diffeomorphism invariance do not require to show that every ideal anti-self-dual connection actually appears in the Uhlenbeck compactification, nor they require the existence of a fine structure (such as that of smooth manifold with corners) on the compactification.

To start our analysis, we fix a unique way to identify the space of unparameterized trajectories

$$
\hat{\mathcal{M}}\left(O_{a}, O_{b}\right)=\mathcal{M}\left(O_{a}, O_{b}\right) / \mathbb{R}
$$


with a subset of the space of parameterized trajectories $\mathcal{M}\left(O_{a}, O_{b}\right)$. It is sufficient to choose the parameterization $x(t)$ of $\hat{x} \in \hat{\mathcal{M}}\left(O_{a}, O_{b}\right)$ which satisfies the equal energy condition

$$
\int_{-\infty}^{0}\left\|\nabla \mathcal{C}_{\rho}(A(t), \psi(t))\right\|_{L^{2}}^{2} d t=\int_{0}^{\infty}\left\|\nabla \mathcal{C}_{\rho}(A(t), \psi(t))\right\|_{L^{2}}^{2} d t
$$

This lifting of $\hat{\mathcal{M}}\left(O_{a}, O_{b}\right)$ to $\mathcal{M}\left(O_{a}, O_{b}\right)$ is unique. In fact, in the family of gradient flows $\left\{[\mathbb{A}, \Psi]^{T}, T \in \mathbb{R}\right\}$ that represent the class $x \in \hat{\mathcal{M}}\left(O_{a}, O_{b}\right)$ there is a unique element which satisfies the equal energy condition (29). The lifting is often referred to as $\mathcal{M}^{\text {bal }}\left(O_{a}, O_{b}\right) \subset \mathcal{M}\left(O_{a}, O_{b}\right)$, the balanced moduli space. We have $\hat{\mathcal{M}}\left(O_{a}, O_{b}\right) \cong \mathcal{M}^{\text {bal }}\left(O_{a}, O_{b}\right)$. In the following, in order to avoid exceeding use of different notation, we shall always write $\hat{\mathcal{M}}\left(O_{a}, O_{b}\right)$, unless we need to make explicit use of the equal energy condition (29), in which case we may recall that $\hat{\mathcal{M}}\left(O_{a}, O_{b}\right)$ is realized by the balanced moduli space $\mathcal{M}^{\text {bal }}\left(O_{a}, O_{b}\right) \subset \mathcal{M}\left(O_{a}, O_{b}\right)$, as in the proof of Theorem 4.9 in Section 4.2 .

\subsection{Convergence theorem.}

The following theorem describes convergence in the moduli space $\hat{\mathcal{M}}\left(O_{a}, O_{b}\right)$, and proves the existence of a compactification, which we denote $\hat{\mathcal{M}}\left(O_{a}, O_{b}\right)^{*}$, obtained by adding broken trajectories.

Theorem 4.1. Consider the moduli space $\hat{\mathcal{M}}\left(O_{a}, O_{b}\right)$, with $\mu\left(O_{a}\right)-\mu\left(O_{b}\right)$ $=k+1, k \geq 0$. The space $\hat{\mathcal{M}}\left(O_{a}, O_{b}\right)$ is precompact. Namely, any sequence $\left[\hat{x}_{i}\right]$ of elements in $\hat{\mathcal{M}}\left(O_{a}, O_{b}\right)$ has a subsequence which either converges in norm to another solution $[\hat{x}] \in \hat{\mathcal{M}}\left(O_{a}, O_{b}\right)$, or converges to a broken trajectory. This means that there are critical orbits $O_{c_{1}}, \ldots, O_{c_{k}}$, with $\mu\left(O_{a}\right) \geq \mu\left(O_{c_{1}}\right)>\ldots>\mu\left(O_{c_{k}}\right) \geq \mu\left(O_{b}\right)$, trajectories $\left[y_{j}\right] \in \mathcal{M}\left(O_{c_{j}}, O_{c_{j+1}}\right)$, and a sequence of real numbers $T_{i_{k}, j} \in \mathbb{R}$ such that the sequence of parameterized trajectories $x_{i_{k}}^{T_{i_{k}, j}}$ converges smoothly on compact sets

$$
x_{i_{k}}^{T_{i_{k}, j}} \rightarrow y_{j}
$$

Here $x_{i_{k}}^{T_{i_{k}}, j}$ denotes the lifting of $\hat{x}_{i_{k}}$ to the space of parameterized trajectories specified by the condition $x_{i_{k}}^{T_{i_{k}, j}}(0)=x_{i_{k}}\left(T_{i_{k}, j}\right)$, where $x_{i_{k}}$ is the equal energy lift of $\hat{x}_{i_{k}}$. 
Proof. The proof of Theorem 4.1 consists of several steps. Suppose that the perturbation $P_{(\mathbb{A}, \Psi)}$ is smooth. Given a sequence of unparameterized trajectories $\left[\hat{x}_{i}\right]$ in $\hat{\mathcal{M}}\left(O_{a}, O_{b}\right)$, choose representatives $x_{i}$ of the corresponding equal energy lift in $\mathcal{M}\left(O_{a}, O_{b}\right)$. By elliptic regularity these can be represented by smooth solutions.

Let $\left(A_{i}( \pm \infty), \psi_{i}( \pm \infty)\right)$ be the asymptotic values of the elements $x_{i}$ at the ends of the cylinder $Y \times \mathbb{R}$. These represent elements $\left[A_{i}( \pm \infty), \psi_{i}( \pm \infty)\right]$ in $O_{a}$ and $O_{b}$ respectively.

Claim 1. There is a subsequence $\left\{x_{i_{k}}\right\}$ that converges smoothly on compact sets to a solution $y$ of the perturbed flow equations.

Proof of Claim 1. We first show that there is a subsequence that converges uniformly on compact sets. By the use of a bootstrapping argument is then possible to improve the convergence to $\mathcal{C}^{\infty}$ on compact sets.

It is useful to recall the following weak version of Arzela'-Ascoli.

Proposition 4.2. Let $K$ be a compact subset of $\mathbb{R}$ and $(X,\|\cdot\|)$ a normed metric space. $S$ is a subset of continuous functions from $K$ to $X$. Suppose the following conditions are satisfied

(i) pointwise bound:

$$
\sup _{f \in S}\|f(t)\|<\infty
$$

for all $t \in K$;

(ii) local equicontinuity: given $\epsilon>0$, for all $t$ in $K$ there exists a neighborhood $V_{t}$ such that, for all $\tau \in V_{t}$

$$
\|f(\tau)-f(t)\|<\epsilon
$$

uniformly in $f \in S$.

Then the set $S$ is uniformly bounded, that is

$$
\sup _{t \in K, f \in S}\|f(t)\|=M<\infty .
$$

We show that the sequence $x_{i}$ satisfies properties (i) and (ii) of Proposition 4.2 . 
Equicontinuity. Choose $t \leq t^{\prime}$. We have

$$
\operatorname{dist}_{L_{1}^{2}}\left(x_{i}(t), x_{i}\left(t^{\prime}\right)\right) \leq K \int_{t}^{t^{\prime}}\left\|\nabla \mathcal{C}_{\rho}\left(x_{i}(s)\right)\right\|_{L_{1}^{2}} d s
$$

where we denote by $\operatorname{dist}_{L_{1}^{2}}\left(x(t), x\left(t^{\prime}\right)\right)$ the quantity

$$
\operatorname{dist}_{L_{1}^{2}}\left(x(t), x\left(t^{\prime}\right)\right)=\left|\int_{t}^{t^{\prime}}\left\|\frac{d}{d s} x(s)\right\|_{L_{1}^{2}} d s\right| .
$$

Since the perturbation satisfies condition (4) of Definition 2.10, we obtain

$$
\begin{aligned}
\operatorname{dist}_{L_{1}^{2}}\left(x_{i}(t), x_{i}\left(t^{\prime}\right)\right) & \leq K\left|t-t^{\prime}\right|^{1 / 2}\left|\int_{t}^{t^{\prime}}\left\|\nabla \mathcal{C}_{\rho}\left(x_{i}(s)\right)\right\|_{L_{1}^{2}}^{2} d s\right|^{1 / 2} \\
& \leq \tilde{K}\left|t-t^{\prime}\right|^{1 / 2}\left|\int_{t-1}^{t^{\prime}+1}\left\|\nabla \mathcal{C}_{\rho}\left(x_{i}(s)\right)\right\|_{L^{2}}^{2} d s\right|^{1 / 2} \\
& \leq \tilde{K} E\left|t-t^{\prime}\right|^{1 / 2}
\end{aligned}
$$

Here the first step comes from the Hölder inequality and the second step from Lemma 6.14 of [48].

Pointwise bound. Fix $t_{0}$. We can assume that

$$
\left\|\nabla \mathcal{C}_{\rho}\left(x_{i}\left(t_{0}\right)\right)\right\|_{L^{2}}>\lambda
$$

for all $i \gg 1$, where $\lambda$ is the constant of Lemma 3.10. In fact, if there is a subsequence $x_{i_{k}}$ such that this condition is not satisfied, then by Lemma 3.10 the elements $x_{i_{k}}\left(t_{0}\right)$ lie in an $\epsilon$-neighborhood of a critical point hence their norms are bounded.

We can therefore choose a sequence $t_{i}$ of real numbers such that

$$
\left\|\nabla \mathcal{C}_{\rho}\left(x_{i}\left(t_{i}\right)\right)\right\|_{L^{2}}=\lambda
$$

and

$$
\left\|\nabla \mathcal{C}_{\rho}\left(x_{i}(t)\right)\right\|_{L^{2}}>\lambda
$$

for $t \in\left(t_{i}, t_{0}\right]$. Lemma 3.10 then implies that there is an element $\left(A_{a}, \psi_{a}\right)$ with $\left[A_{a}, \psi_{a}\right] \in O_{a}$ and there are gauge transformations $\lambda_{i}$ such that

$$
\operatorname{dist}_{L_{1}^{2}}\left(\lambda_{i} x_{i}\left(t_{i}\right),\left(A_{a}, \psi_{a}\right)\right) \leq \epsilon
$$


for all $i$, and therefore we can write

$$
\begin{aligned}
& \operatorname{dist}_{L_{1}^{2}}\left(\lambda_{i} x_{i}\left(t_{0}\right),\left(A_{a}, \psi_{a}\right)\right) \\
& \leq \operatorname{dist}_{L_{1}^{2}}\left(\lambda_{i} x_{i}\left(t_{i}\right),\left(A_{a}, \psi_{a}\right)\right)+\operatorname{dist}_{L_{1}^{2}}\left(\lambda_{i} x_{i}\left(t_{i}\right), \lambda_{i} x_{i}\left(t_{0}\right)\right) \\
& \leq \epsilon+K \int_{t_{i}}^{t_{0}}\left\|\nabla \mathcal{C}_{\rho}\left(\lambda_{i} x_{i}(t)\right)\right\|_{L_{1}^{2}} d t .
\end{aligned}
$$

Using the inequality

$$
\left\|\nabla \mathcal{C}_{\rho}\left(\lambda_{i} x_{i}(t)\right)\right\|_{L_{1}^{2}} \leq \frac{\left\|\nabla \mathcal{C}_{\rho}\left(\lambda_{i} x_{i}(t)\right)\right\|_{L_{1}^{2}}^{2}}{\left\|\nabla \mathcal{C}_{\rho}\left(\lambda_{i} x_{i}(t)\right)\right\|_{L^{2}}} \leq \frac{\left\|\nabla \mathcal{C}_{\rho}\left(\lambda_{i} x_{i}(t)\right)\right\|_{L_{1}^{2}}^{2}}{\lambda},
$$

for $t \in\left[t_{i}, t_{0}\right]$, and Lemma 6.14 of [48], we obtain

$$
\operatorname{dist}_{L_{1}^{2}}\left(\lambda_{i} x_{i}\left(t_{0}\right),\left(A_{a}, \psi_{a}\right)\right) \leq \epsilon+\frac{\tilde{K}}{\lambda} \int_{t_{i}-1}^{t_{0}+1}\left\|\nabla \mathcal{C}_{\rho}\left(\lambda_{i} x_{i}(t)\right)\right\|_{L^{2}}^{2} \leq \epsilon+\tilde{K} E / \lambda .
$$

Now Proposition 4.2 implies that, given any compact set $K \subset \mathbb{R}$, we have

$$
\sup _{t \in K}\left\|\lambda_{i} x_{i}(t)\right\|_{L_{1}^{2}(Y \times\{t\})}=M<\infty
$$

uniformly in $i$.

For simplicity of notation we refer in the following to the gauge transformed sequence $\lambda_{i} x_{i}$ simply as $x_{i}$.

From the uniform estimate (30) and from the equations (18) and (19) we obtain a uniform bound of the $L^{2}$ norms of $\frac{d}{d t} x_{i}(t)$ on $Y \times K$ :

$$
\left\|\frac{d}{d t} x_{i}(t)\right\|_{L^{2}(Y \times\{t\})} \leq C\left\|x_{i}(t)\right\|_{L_{1}^{2}(Y \times\{t\})},
$$

hence the left hand side is bounded uniformly with respect to $t \in K$ and $i$. We use the fact that the perturbations $P_{\left(\mathbb{A}_{i}, \Psi_{i}\right)}$ are uniformly bounded with respect to $\left(\mathbb{A}_{i}, \Psi_{i}\right)$ according to condition (2) of Definition 2.10.

In the following we shall always consider the set $K$ to be some large interval $[-T, T]$. The previous estimates provide a uniform bound of the norms $\left\|x_{i}\right\|_{L_{1}^{2}(Y \times[-T, T])}$. In fact, we have

$$
\left\|x_{i}\right\|_{L_{1}^{2}(Y \times[-T, T])}^{2} \leq 2 T M+2 T C M .
$$

In order to bound the higher Sobolev norms we need the following gauge fixing condition. Up to gauge transformations $\lambda_{i}$ in the group $\tilde{\mathcal{G}}_{k+1}(Y \times$ $[-T, T])$, it is possible to make $\mathbb{A}_{i}-\mathbb{A}_{0}$ into a sequence of co-closed 1-forms. 
We have elliptic estimates for the domain $Y \times[-T, T]$,

$$
\begin{aligned}
\left\|\mathbb{A}_{i}-\mathbb{A}_{0}\right\|_{L_{k}^{2}(Y \times[-T, T])} \leq & C\left(\left\|\left(d^{*}+d^{+}\right)\left(\mathbb{A}_{i}-\mathbb{A}_{0}\right)\right\|_{L_{k-1}^{2}\left(Y \times\left[-T^{\prime}, T^{\prime}\right]\right)}\right. \\
& \left.+\left\|\mathbb{A}_{i}-\mathbb{A}_{0}\right\|_{L_{k-1}^{2}(Y \times[-T, T])}\right)
\end{aligned}
$$

and

$$
\left\|\Psi_{i}\right\|_{L_{k}^{2}(Y \times[-T, T])} \leq C\left(\left\|\nabla_{A} \Psi_{i}\right\|_{L_{k-1}^{2}\left(Y \times\left[-T^{\prime}, T^{\prime}\right]\right)}+\left\|\Psi_{i}\right\|_{L_{k-1}^{2}(Y \times[-T, T])}\right),
$$

where $\left[-T^{\prime}, T^{\prime}\right]$ is any strictly smaller interval contained in $[-T, T]$. We also have an estimate given by the curvature equation:

$$
\left\|F_{\mathbb{A}_{i}}^{+}\right\|_{L_{k}^{2}(Y \times[-T, T])} \leq\left\|\Psi_{i} \cdot \bar{\Psi}_{i}\right\|_{L_{k}^{2}(Y \times[-T, T])}+\left\|i \mu+P_{\left(\mathbb{A}_{i}, \Psi_{i}\right)}\right\|_{L_{k}^{2}(Y \times[-T, T])} .
$$

These provide a uniform bound on the $L_{k}^{2}$-norms of the $\left(\mathbb{A}_{i}, \Psi_{i}\right)$ on a smaller $Y \times\left[-T^{\prime}, T^{\prime}\right]$. By the Sobolev embeddings, this implies that on a smaller $Y \times$ $\left[-T^{\prime \prime}, T^{\prime \prime}\right]$ there is a subsequence $x_{i_{k}}=\left(\mathbb{A}_{i_{k}}, \Psi_{i_{k}}\right)$ that converges uniformly with all derivatives.

This completes the proof of Claim 1: there is a subsequence $x_{i_{k}}$ that converges smoothly on compact sets to a solution $y=(\mathbb{A}, \Psi)$ of $(18)$ and (19) on $Y \times \mathbb{R}$.

The limit $y$ is of finite energy, hence it defines an element of some moduli space $\mathcal{M}\left(O_{c}, O_{d}\right)$ according to Corollary 3.11 .

For simplicity of notation, assume that the sequence $x_{i}$ itself converges to $y$ smoothly on compact sets.

Claim 2. If the limit $y$ is an element of $\mathcal{M}\left(O_{a}, O_{b}\right)$ then the convergence $x_{i} \rightarrow y$ is strong in the $L_{k, \delta}^{2}$ norm.

Proof of Claim 2. There exists a $T_{0}$ such that for all $t \leq-T_{0}$ we have

$$
\operatorname{dist}_{L_{1}^{2}}\left(y(t),\left(\tilde{A}_{a}, \tilde{\psi}_{a}\right)\right) \leq \epsilon / 2,
$$

for some element $\left(\tilde{A}_{a}, \tilde{\psi}_{a}\right)$ with $\left[\tilde{A}_{a}, \tilde{\psi}_{a}\right] \in O_{a}$. For $i \gg 1$ we also have

$$
\operatorname{dist}_{L_{1}^{2}}\left(x_{i}\left(-T_{0}\right), y\left(-T_{0}\right)\right) \leq \epsilon / 2,
$$

hence

$$
\operatorname{dist}_{L_{1}^{2}}\left(x_{i}\left(-T_{0}\right),\left(\tilde{A}_{a}, \tilde{\psi}_{a}\right)\right) \leq \epsilon .
$$

This implies that $\left(\tilde{A}_{a}, \tilde{\psi}_{a}\right)$ is the same as the element $\left(A_{a}, \psi_{a}\right)$. Moreover, the exponential decay property ensures that we have

$$
\operatorname{dist}_{L_{1}^{2}}\left(x_{i}(t),\left(A_{a}, \psi_{a}\right)\right) \leq \epsilon
$$


for all $t \leq-T_{0}$. This, together with the uniform convergence on compact sets, implies that the convergence is uniform on all $Y \times \mathbb{R}$. Thus, we have a uniform exponential decay towards the endpoints, hence convergence in the $L_{1, \delta}^{2}$ norm.

Notice that in this case we can ensure that there is a unique $T_{0}$ such that (31) is satisfied for all $t \leq-T_{0}$, whereas, in the proof of the exponential decay, the interval $\left(-\infty, T_{0}\right]$ depends on the solution $(A(t), \psi(t))$.

To improve the convergence to $L_{k, \delta}^{2}$ we need to choose suitable gauge transformations.

Lemma 4.3. There exist gauge transformations $\lambda_{i}$ in $\mathcal{G}_{2, \delta}^{0}\left(O_{a}, O_{b}\right)$ such that the forms $\lambda_{i}\left(\mathbb{A}_{i}-\mathbb{A}_{0}\right)$ are co-closed, that is

$$
d_{\delta}^{*} \lambda_{i}\left(\mathbb{A}_{i}-\mathbb{A}_{0}\right)=0 .
$$

Proof of Lemma. Let $\alpha_{i}=\mathbb{A}_{i}-\mathbb{A}_{0}$. The element $e_{\delta / 2} d^{*} e_{-\delta} \alpha_{i}$ is $L^{2}$ orthogonal to the kernel of the Laplacian $\Delta_{\delta / 2}$. Thus we can define the elements

$$
g_{i}=e_{-\delta / 2} \Delta_{\delta / 2}^{-1}\left(e_{\delta / 2} d^{*} e_{-\delta} \alpha_{i}\right)
$$

with

$$
\Delta_{\delta / 2}^{-1}: \operatorname{Ker}\left(\Delta_{\delta / 2}\right)_{L_{1, \delta}^{2}}^{\perp} \rightarrow \operatorname{Ker}\left(\Delta_{\delta / 2}\right)_{L_{2, \delta}^{2}}^{\perp}
$$

These are elements in the $L_{2, \delta}^{2}$ completion of the Lie algebra hence they define gauge transformations $\lambda_{i} \in \mathcal{G}_{2, \delta}^{0}\left(O_{a}, O_{b}\right)$. We have

$$
\lambda_{i}\left(\mathbb{A}_{i}-\mathbb{A}_{0}\right)=\beta_{i}=\alpha_{i}-d g_{i} .
$$

Thus, we can compute $d_{\delta}^{*} \beta_{i}$ as

$$
\begin{aligned}
d_{\delta}^{*} \beta_{i} & =d_{\delta}^{*} \alpha_{i}-d_{\delta}^{*} d e_{-\delta / 2} \Delta_{\delta / 2}^{-1}\left(e_{\delta / 2} d^{*} e_{-\delta} \alpha_{i}\right) \\
& =d_{\delta}^{*} \alpha_{i}-e_{\delta / 2}\left(d_{\delta / 2}^{*} d_{\delta / 2}\right) \Delta_{\delta / 2}^{-1} e_{\delta / 2} d^{*} e_{\delta} \alpha_{i} \\
& =d_{\delta}^{*} \alpha_{i}-d_{\delta}^{*} \alpha_{i}=0 .
\end{aligned}
$$

This completes the proof of the Lemma.

Now we can use the elliptic estimates to improve the convergence to $L_{k, \delta}^{2}$. This completes the proof of Claim 2.

Thus, we see that broken trajectories arise when the limit $y$ is in some $\mathcal{M}\left(O_{c}, O_{d}\right)$ where either $O_{c} \neq O_{a}$ or $O_{d} \neq O_{b}$. These are constrained by the condition

$$
\mathcal{C}_{\rho}(a) \geq \mathcal{C}_{\rho}(c) \geq \mathcal{C}_{\rho}(d) \geq \mathcal{C}_{\rho}(b)
$$


Without loss of generality, assume that $y$ is in $\mathcal{M}\left(O_{a}, O_{d}\right)$ and $\mathcal{C}_{\rho}(a)>$ $\mathcal{C}_{\rho}(d)>\mathcal{C}_{\rho}(b)$.

There exists a sequence of real numbers $T_{i}$ such that $\mathcal{C}_{\rho}\left(x_{i}\left(T_{i}\right)\right)=\alpha$, with $\alpha$ satisfying $\mathcal{C}_{\rho}(d)>\alpha>\mathcal{C}_{\rho}(b)$. Consider the reparameterization $x_{i}^{T_{i}}(t)=x_{i}\left(t+T_{i}\right)$. This is another possible lifting of $\hat{x}_{i}$ to the parameterized trajectories (which does not satisfy the equal energy condition). We can apply the result of Claim 1 to the sequence $x_{i}^{T_{i}}$ and we obtain a subsequence that converges smoothly on compact sets to a solution $\tilde{y}$ of the equations (18) and (19) on $Y \times \mathbb{R}$. Now the asymptotic values $\tilde{y}( \pm \infty)$ are constrained by

$$
\mathcal{C}_{\rho}(d) \geq \mathcal{C}_{\rho}(\tilde{y}(-\infty)) \geq \mathcal{C}_{\rho}(\tilde{y}(\infty)) \geq \mathcal{C}_{\rho}(b)
$$

The process can be repeated: at each step we either get strong convergence or another broken trajectory. If we start with relative index $k+1$, the process ends at most at the order $k$, in fact the condition

$$
\mu\left(O_{a}\right) \geq \mu\left(O_{c}\right)>\mu\left(O_{d}\right) \geq \mu\left(O_{b}\right)
$$

has to be satisfied for dimensional reasons.

Corollary 4.4. Notice that, in particular, if $\mu\left(O_{a}\right)-\mu\left(O_{b}\right)=1$ with $O_{a} a$ free orbit, or if $\mu\left(O_{a}\right)-\mu\left(O_{b}\right)=2$, with $O_{a}=\theta$ the unique fixed point, the previous argument shows that $\hat{\mathcal{M}}\left(O_{a}, O_{b}\right)$ is already compact, $\hat{\mathcal{M}}\left(O_{a}, O_{b}\right)=$ $\hat{\mathcal{M}}\left(O_{a}, O_{b}\right)^{*}$.

A brief notational remark about the case $b^{1}(Y)>0$ :

Remark 4.5. In the case of a three-manifold with $b^{1}(Y)>0$, the SeibergWitten Floer homology considered in this paper differs from the construction of [38], where the connected component of the full gauge group $\tilde{\mathcal{G}}(Y)$ is considered. The relation is illustrated in the following.

With our notation, we have $\tilde{\mathcal{G}}(Y)$, the full gauge group on $Y$, and $\mathcal{G}(Y)$, the group of gauge transformations on $Y$ satisfying the condition (2). We have another possible choice of an even smaller subgroup, the identity component $\mathcal{G}_{c}(Y)$ of the gauge group. These all have subgroups of base point preserving gauge transformations, $\tilde{\mathcal{G}}^{0}(Y), \mathcal{G}^{0}(Y), \mathcal{G}_{c}^{0}(Y)$.

Thus, we have configuration spaces $\tilde{\mathcal{B}}^{0}, \mathcal{B}^{0}$, and $\mathcal{B}_{c}^{0}$. The map $\mathcal{B}_{c}^{0} \rightarrow \tilde{\mathcal{B}}^{0}$ has fiber $H^{1}(Y, \mathbb{Z})$, and the map $\mathcal{B}^{0} \rightarrow \tilde{\mathcal{B}}^{0}$ has fiber the group $H$

$$
H=\left\{h \in H^{1}(Y, \mathbb{Z}) \mid\left\langle c_{1}(L) \cup h,[Y]\right\rangle=0\right\} .
$$


The group $H$ is the kernel of the morphism

$$
\begin{gathered}
\xi: H^{1}(Y, \mathbb{Z}) \rightarrow \mathbb{Z}, \\
\xi(h)=\left\langle c_{1}(L) \cup h,[Y]\right\rangle .
\end{gathered}
$$

The quotient $H^{1}(Y, \mathbb{Z}) / H$ is either trivial or $\mathbb{Z}$. It is trivial only when $c_{1}(L)=0$ rationally, that is in the case of Remark 2.18 which will be considered separately in [42]. Thus we have the following diagram

$$
\begin{aligned}
\mathcal{B}_{c}^{0}(Y) & =\mathcal{A}(Y) / \mathcal{G}_{c}^{0}(Y) \\
\mathcal{B}^{0}(Y)=\mathcal{A}(Y) / \mathcal{G}^{0}(Y)_{Z=H^{1}(Y, Z) / H} & H^{1}(Y, Z) \\
\tilde{\mathcal{B}}^{0}(Y) & =\mathcal{A}(Y) / \tilde{\mathcal{G}}^{0}(Y)
\end{aligned}
$$

As we are about to see, the choice of $\tilde{\mathcal{B}}^{0}(Y)$ gives rise to a periodic Floer homology of periodicity

$$
l=g . c . d\left\{\left\langle c_{1}(L) \cup h,[Y]\right\rangle, h \in H^{1}(Y, \mathbb{Z})\right\} .
$$

The choice of $\mathcal{B}^{0}(Y)$, that was suggested to us by R.G. Wang, represents the minimal cover of $\tilde{\mathcal{B}}^{0}(Y)$ that gives rise to a $\mathbb{Z}$-graded Floer homology. The choice of $\mathcal{B}_{c}^{0}(Y)$ also gives rise to a $\mathbb{Z}$-graded complex.

In [38] the Floer homology is constructed using the group $\mathcal{G}_{c}(Y)$. The relation to our construction here is as follows. Let $\left\{O_{a}^{h}\right\}_{h \in H}$ be the family of distinct critical orbits in $\mathcal{B}_{c}^{0}$ that correspond to a critical orbit $O_{a}$ in $\mathcal{B}^{0}$. Notice that in $\mathcal{B}_{c}^{0}$ the relative Morse index satisfies

$$
\mu\left(O_{a}\right)-\mu\left(O_{a}^{h}\right)=\int_{Y} c_{1}(L) \wedge h=0 .
$$

We have the configuration space of flow lines

$$
\mathcal{A}_{k, \delta}\left(O_{a}^{h}, O_{b}^{h^{\prime}}\right)=\bigcup_{\lambda \in U(1)} \mathcal{A}_{k, \delta}\left(\lambda\left(A_{a}, \psi_{a}\right), \lambda\left(A_{b}, \psi_{b}\right)\right),
$$

with $\left(A_{a}, \psi_{a}\right)$ and $\left(A_{b}, \psi_{b}\right)$ representatives of elements $\left[A_{a}, \psi_{a}\right]$ and $\left[A_{b}, \psi_{b}\right]$ in $O_{a}^{h}$ and $O_{b}^{h^{\prime}}$ respectively, in the space $\mathcal{B}_{c}^{0}(Y)$. The space $\mathcal{A}_{k, \delta}\left(O_{a}^{h}, O_{b}^{h^{\prime}}\right)$ is acted upon by the gauge group of gauge transformations in $\tilde{\mathcal{G}}(Y \times \mathbb{R})$ that decay to elements of $G_{a}$ and $G_{b}$ respectively. We can form the quotient space $\mathcal{B}_{c}^{0}\left(O_{a}^{h}, O_{b}^{h^{\prime}}\right)$. 
We can consider the moduli spaces of flow lines $\mathcal{M}\left(O_{a}^{h}, O_{b}^{h^{\prime}}\right)$ in the space $\mathcal{B}_{c}^{0}\left(O_{a}, O_{b}\right)$. In the analysis of the boundary structure of $\mathcal{M}\left(O_{a}^{h}, O_{b}^{h^{\prime}}\right)$, there may be a question of whether a sequence of elements $\left[x_{i}\right]$ of $\mathcal{M}\left(O_{a}^{h}, O_{b}^{h^{\prime}}\right)$ can converge to a solution $y$ that lives in another $\mathcal{M}\left(O_{a}^{h}, O_{b}^{h^{\prime}}\right)$ (for different $h$ and $h^{\prime}$ ). Claim 2 of Theorem 4.1 rules out this possibility. Thus, a nice compactification of the moduli spaces $\mathcal{M}\left(O_{a}^{h}, O_{b}^{h^{\prime}}\right)$ can be achieved in this setting as well, for all manifolds with $b^{1}(Y)>0$ and $c_{1}(L) \neq 0$. There is, therefore, a well defined associated Seiberg-Witten Floer homology which is finer than the one we are considering here.

On the other hand we can also consider critical orbits $\tilde{O}_{a}$ in the configuration space $\tilde{\mathcal{B}}^{0}(Y)$. In this case the relative Morse index is not well defined, in fact the spectral flow between two gauge equivalent orbits satisfies

$$
\mu\left(\lambda \tilde{O}_{a}\right)-\mu\left(\tilde{O}_{a}\right)=\int_{Y} c_{1}(L) \wedge h(\lambda),
$$

with $h(\lambda)=\lambda^{-1} d \lambda$. Thus the grading of the Floer complex is defined only up to a periodicity of

$$
l=g . c . d\left\{\left\langle c_{1}(L) \cup h(\lambda),[Y]\right\rangle\right\} .
$$

Therefore in the configuration space $\tilde{\mathcal{B}}\left(\tilde{O}_{a}, \tilde{O}_{b}\right)$ obtained as a quotient of

$$
\mathcal{A}_{k, \delta}\left(\tilde{O}_{a}, \tilde{O}_{b}\right)=\bigcup_{\lambda \in U(1)} \mathcal{A}_{k, \delta}\left(\lambda\left(A_{a}, \psi_{a}\right), \lambda\left(A_{b}, \psi_{b}\right)\right),
$$

with $\left(A_{a}, \psi_{a}\right)$ and $\left(A_{b}, \psi_{b}\right)$ representatives of elements $\left[A_{a}, \psi_{a}\right]$ and $\left[A_{b}, \psi_{b}\right]$ in $\tilde{O}_{a}$ and $\tilde{O}_{b}$ respectively, in the space $\tilde{\mathcal{B}}^{0}(Y)$. The space $\mathcal{A}_{k, \delta}\left(\tilde{O}_{a}, \tilde{O}_{b}\right)$ is acted upon by the gauge group $\mathcal{G}_{k+1, \delta}\left(\tilde{O}_{a}, \tilde{O}_{b}\right)$ of gauge transformations in $\tilde{\mathcal{G}}(Y \times \mathbb{R})$ that decay to elements of $G_{a}$ and $G_{b}$.

Now the space $\mathcal{M}\left(\tilde{O}_{a}, \tilde{O}_{b}\right)$ has infinitely many components of virtual dimensions

$$
\mu\left(O_{a}\right)-\mu\left(O_{b}\right)+k l,
$$

with $k \in \mathbb{Z}$. Each component has uniformly bounded energy, hence it has a compactification by boundary strata of broken trajectories.

The Floer homology defined this way, namely with generators in $\tilde{\mathcal{M}}$ and with the boundary operator defined by counting the components of minimal energy in $\mathcal{M}\left(\tilde{O}_{a}, \tilde{O}_{b}\right)$, is finitely generated and $\mathbb{Z}_{l}$-graded. There are various other ways of defining the Floer homology for $b^{1}(Y)>0$. For instance, a way of obtaining a $\mathbb{Z}$-graded Floer homology which is also finitely generated (again for $c_{1}(L) \neq 0$ ) is by a filtration as in [23] or [34]. The comparison 
between these different versions will be discussed in [42]. For the purpose of this paper we shall only consider the Seiberg-Witten Floer homology defined by the choice of the gauge group $\mathcal{G}$.

\subsection{Gluing theorem.}

Theorem 4.1 proves that lower dimensional moduli spaces appear naturally in the compactification of the spaces $\hat{\mathcal{M}}\left(O_{a}, O_{b}\right)$. In the rest of this section we describe a gluing formula, thus proving that all the broken trajectories in the ideal boundary which break through one intermediate critical point actually occur as codimension one strata in $\hat{\mathcal{M}}\left(O_{a}, O_{b}\right)^{*}$. The case of multiply broken trajectories is analyzed in Section 4.3.

In order to show that the gluing construction is well defined over the gauge classes, we need a preliminary discussion of slices of the gauge action.

Definition 4.6. Let $\Gamma=(\mathbb{A}, \Psi)$ be an element in $\mathcal{A}_{k, \delta}\left(O_{a}, O_{b}\right)$. The slice $\mathcal{S}_{\Gamma}$ at $\Gamma$ for the action of the gauge group $\mathcal{G}_{k+1, \delta}^{0}(Y \times \mathbb{R})$, is the set of elements $(\mathbb{A}, \Psi)+(\alpha, \phi)$ in the configuration space $\mathcal{A}_{k, \delta}\left(O_{a}, O_{b}\right)$ with the following properties. The element $(\alpha, \phi)$ satisfies

$$
(\alpha, \phi) \in \operatorname{Ker}\left(G_{(\mathbb{A}, \Psi)}^{*}\right),
$$

where $G_{(\mathbb{A}, \Psi)}^{*}$ is the adjoint with respect to the $L_{\delta}^{2}$ norm of the linearization of the gauge group action. There is a $T_{0}>>0$, such that, on $Y \times\left[T_{0}, \infty\right)$ we have

$$
(\alpha, \phi)=(\tilde{\alpha}, \tilde{\phi})+\left(e_{b}^{-}\right)^{*}\left(A_{1}, \psi_{1}\right),
$$

and on $Y \times\left(-\infty,-T_{0}\right]$ we have

$$
(\alpha, \phi)=(\tilde{\alpha}, \tilde{\phi})+\left(e_{a}^{+}\right)^{*}\left(A_{0}, \psi_{0}\right),
$$

with $(\tilde{\alpha}, \tilde{\phi})$ is in $L_{k, \delta}^{2}$ on $Y \times \mathbb{R}$. The elements $\left(A_{0}, \psi_{0}\right)$ and $\left(A_{1}, \psi_{1}\right)$ are representatives in the configuration space $\mathcal{A}$ over $Y$ of elements in the orbits $O_{a}$ and $O_{b}$.

The following Lemma shows that there is a uniform choice of slices for the gluing construction, cf. Lemma 2.1.4 and 2.1.5 of [46].

Lemma 4.7. Given $\Gamma=(\mathbb{A}, \Psi)$ in the space $\mathcal{A}_{k, \delta}\left(O_{a}, O_{b}\right)$, consider $\mu_{(\mathbb{A}, \Psi)}$, the first positive eigenvalue of the operator $G_{(\mathbb{A}, \Psi)}^{*} G_{(\mathbb{A}, \Psi)}$. There is a ball of radius $r(\mu)$ around $\Gamma$ in the configuration space $\mathcal{A}_{k, \delta}\left(O_{a}, O_{b}\right)$ such that the intersection of the ball with the slice $\mathcal{S}_{\Gamma}$ embeds into $\mathcal{B}_{k, \delta}^{0}\left(O_{a}, O_{b}\right)$. 
Moreover, there is a radius $r$ such that we have an embedding

$$
\mathcal{S}_{(\mathbb{A}, \Psi)} \cap B_{r}(\mathbb{A}, \Psi) \hookrightarrow \mathcal{B}_{k, \delta}^{0}\left(O_{a}, O_{b}\right)
$$

for all $[\mathbb{A}, \Psi]$ in $\mathcal{M}\left(O_{a}, O_{b}\right)$.

Proof. The claim that the radius can be taken to be $r=r(\mu)$ follows from Lemma 10.3.1 of [47] (cf. similar arguments in Lemma 2.1.5 of [46]). We need to show that there is a uniform lower bound for $\mu_{(\mathbb{A}, \Psi)}$ as $[\mathbb{A}, \Psi]$ varies in $\mathcal{M}\left(O_{a}, O_{b}\right)$. Consider a sequence of elements $x_{i}$ in $\mathcal{M}\left(O_{a}, O_{b}\right)$. Theorem 4.1 implies that a subsequence converges smoothly on compact sets to a limit $y$ that defines an element in some $\mathcal{M}\left(O_{c}, O_{d}\right)$. Since we are considering the adjoint operator $G_{(\mathbb{A}, \Psi)}^{*}$ with respect to the $L_{\delta}^{2}$ norm, for a generic choice of metric and perturbation, we have $\mu_{y}>0$, as discussed in Lemma 3.13. We also have $\mu_{x_{i}} \rightarrow \mu_{y}$, and this provides the uniform bound. In fact, according to Theorem 4.1, only finitely many different components $\mathcal{M}\left(O_{c}, O_{d}\right)$ can appear in the boundary of $\mathcal{M}\left(O_{a}, O_{b}\right)$.

The following result is also useful in constructing a gluing map at the level of gauge classes, cf. Lemma 2.1.8 of [46].

Lemma 4.8. Consider a compact subset $K \subset \mathcal{M}\left(O_{a}, O_{b}\right)$. Then there is a radius $r$, such that, for every $[\Gamma] \in K$, there is a $U(1)$-invariant submanifold $V_{\Gamma} \subset \mathcal{A}_{k, \delta}\left(O_{a}, O_{b}\right)$ with the following properties. Suppose given any solution $(\mathbb{A}, \Psi)$ of equations (20) and (21) that is in the ball of radius $r$ around $\Gamma$. Then $V_{\Gamma}$ contains an element gauge equivalent to $(\mathbb{A}, \Psi)$. Moreover, the quotient by the action of the gauge group $\mathcal{G}_{k+1, \delta}^{0}(Y \times \mathbb{R})$ induces a map

$$
V_{\Gamma} \rightarrow \mathcal{M}\left(O_{a}, O_{b}\right)
$$

that is a diffeomorphism on a neighborhood of $[\Gamma]$. Moreover, there is a $T_{0}$ such that, for all $T \geq T_{0}$ we can find families of gauge transformations

$$
\left\{\lambda_{x}^{ \pm} \mid x \in V_{\Gamma}\right\}
$$

such that the elements in the set

$$
W_{\Gamma}^{ \pm}=\left\{\lambda_{x}^{ \pm}(x) \mid x \in V_{\Gamma}\right\}
$$

are in a temporal gauge on $(-\infty,-T]$ in the case of $W_{\Gamma}^{-}$and on $[T, \infty)$ in the case of $W_{\Gamma}^{+}$. The set $W_{\Gamma}^{ \pm}$has the same properties of $V_{\Gamma}$, namely the quotient with respect to the gauge action induces a diffeomorphism

$$
W_{\Gamma}^{ \pm} \rightarrow \mathcal{M}\left(O_{a}, O_{b}\right)
$$


on a neighborhood of $\Gamma$.

Proof. It is sufficient to take $V_{\Gamma}$ to be the intersection of the space of solutions to (20) and (21) in $\mathcal{A}_{k, \delta}\left(O_{a}, O_{b}\right)$ with the slice $\mathcal{S}_{\Gamma}$. According to Lemma 4.7, there is a radius $r_{1}$ such that the intersection of this set with the ball of radius $r_{1}$ around $\Gamma$ embeds in $\mathcal{M}\left(O_{a}, O_{b}\right)$. In general, elements of $V_{\Gamma}$ are not in a temporal gauge, however, following Corollary 3.1.7 of [47], there exists a. constant $C$ independent of $x \in V_{\Gamma}$ such that we obtain $\left\|\lambda_{x}^{ \pm}(x)\right\| \leq C\|x\|$, and the elements in the set

$$
W_{\Gamma}^{ \pm}=\left\{\lambda_{x}^{ \pm}(x) \mid x \in V_{\Gamma}\right\}
$$

are in a temporal gauge on $(-\infty,-T]$ and on $[T, \infty)$, respectively. This follows from the exponential decay of solutions towards the endpoints. Upon rescaling the radius $r$ to $r / C$ the properties stated for $V_{\Gamma}$ hold for $W_{\Gamma}^{ \pm}$. The constant $C$ depends only on the set $K$, cf. Lemma 2.1.8 and Proposition 2.6 .4 of $[46]$.

Now we can introduce the gluing construction. This identifies the codimension one boundary in the compactification $\mathcal{M}\left(O_{a}, O_{b}\right)^{*}$.

Theorem 4.9. Suppose given $O_{a}, O_{b}$ and $O_{c}$ in $\mathcal{M}^{0}$ with $\mu\left(O_{a}\right)>\mu\left(O_{b}\right)>$ $\mu\left(O_{c}\right)$. Assume that $b$ is irreducible. Then, given a compact set

$$
K \subset \mathcal{M}\left(O_{a}, O_{b}\right) \times O_{b} \mathcal{M}\left(O_{b}, O_{c}\right),
$$

there are a lower bound $T_{0}(K)>0$ and a smooth map

$$
\begin{gathered}
\#: K \times\left[T_{0}, \infty\right) \rightarrow \mathcal{M}\left(O_{a}, O_{c}\right) \\
\left(\left(\mathbb{A}_{1}, \Psi_{1}\right),\left(\mathbb{A}_{2}, \Psi_{2}\right), T\right) \mapsto\left(\mathbb{A}_{1} \#_{T} \mathbb{A}_{2}, \Psi_{1} \#_{T} \Psi_{2}\right),
\end{gathered}
$$

such that $\#_{T}$ is an embedding for all $T>T_{0}(K)$. The gluing map \# induces a smooth embedding

$$
\hat{\#}: \hat{K} \times\left[T_{0}, \infty\right) \rightarrow \hat{\mathcal{M}}\left(O_{a}, O_{c}\right),
$$

where

$$
\hat{K} \subset \hat{\mathcal{M}}\left(O_{a}, O_{b}\right) \times O_{b} \hat{\mathcal{M}}\left(O_{b}, O_{c}\right) .
$$

This local diffeomorphism is compatible with the orientation, where the orientation on $K \times\left[T_{0}, \infty\right)$ is induced by the product orientation on

$$
\mathcal{M}\left(O_{a}, O_{b}\right) \times \mathbb{R} \times \mathcal{M}\left(O_{b}, O_{c}\right) .
$$


If $b$ is reducible, then $O_{b}$ is just a point and there is a similar orientation preserving local diffeomorphism

$$
\hat{\#}: \hat{\mathcal{M}}\left(O_{a}, b\right) \times \hat{\mathcal{M}}\left(b, O_{c}\right) \times\left[T_{0}, \infty\right) \rightarrow \hat{\mathcal{M}}\left(O_{a}, O_{c}\right) .
$$

Moreover, any sequence of trajectories in $\hat{\mathcal{M}}\left(O_{a}, O_{c}\right)$ converging to a broken trajectory in $\hat{\mathcal{M}}\left(O_{a}, O_{b}\right) \times O_{b} \hat{\mathcal{M}}\left(O_{b}, O_{c}\right)$ lies eventually in the image of the gluing map.

In order to prove Theorem 4.9, we first define a pre-gluing map $\#_{T}^{0}$. This provides an approximate solution via the following construction. Consider classes $[x]=\left[\mathbb{A}_{1}, \Psi_{1}\right]$ and $[y]=\left[\mathbb{A}_{2}, \Psi_{2}\right]$ in $\mathcal{M}\left(O_{a}, O_{b}\right)$ and $\mathcal{M}\left(O_{b}, O_{c}\right)$ respectively. Let

$$
\left(A_{1}(t), \psi_{1}(t)\right) \in \mathcal{A}_{k, \delta}\left(O_{a}, O_{b}\right), \quad\left(A_{2}(t), \psi_{2}(t)\right) \in \mathcal{A}_{k, \delta}\left(O_{b}, O_{c}\right)
$$

be temporal gauge representatives of $[x]$ and $[y]$.

Choose slices

$$
\mathcal{S}_{\Gamma_{a b}} \subset \mathcal{A}_{k, \delta}\left(O_{a}, O_{b}\right)
$$

and

$$
\mathcal{S}_{\Gamma_{b c}} \subset \mathcal{A}_{k, \delta}\left(O_{b}, O_{c}\right),
$$

determined respectively by the elements

$$
\Gamma_{a b} \in \mathcal{A}_{k, \delta}\left(\left(A_{a}, \psi_{a}\right),\left(A_{b}, \psi_{b}\right)\right)
$$

and

$$
\Gamma_{b c} \in \mathcal{A}_{k, \delta}\left(\left(A_{b}, \psi_{b}\right),\left(A_{c}, \psi_{c}\right)\right) .
$$

We choose them so that there is a representative $x$ in the ball of radius $r$ in $\mathcal{A}_{k, \delta}\left(O_{a}, O_{b}\right)$ centered at $\Gamma_{a b}$ and a representative $y$ in the ball of radius $r$ in $\mathcal{A}_{k, \delta}\left(O_{b}, O_{c}\right)$ centered at $\Gamma_{b c}$. According to Lemma 4.8, there are gauge transformations $\lambda_{1}^{+}$and $\lambda_{2}^{-}$such that we have

$$
\lambda_{1}^{+} x \in W_{\Gamma_{a b}}^{+}, \quad \lambda_{2}^{-} y \in W_{\Gamma_{b c}}^{-} .
$$

Thus, for $|t|>T_{0}$ and for all $T \geq T_{0}$, we can write $\lambda_{1}^{+} x(t)=\lambda_{b}\left(A_{b}, \psi_{b}\right)+$ $\left(\alpha_{1}(t), \phi_{1}(t)\right)$ and $\lambda_{2}^{-} y(t)=\lambda_{b}\left(A_{b}, \psi_{b}\right)+\left(\alpha_{2}(t), \phi_{2}(t)\right)$. Here we have $\lambda_{b} \in$ $\mathcal{G}(Y)$ and $\lambda_{b}\left(A_{b}, \psi_{b}\right)$ is a representative of an element in the orbit $O_{b}$ that satisfies

$$
\lim _{t \rightarrow \infty}\left(A_{1}(t), \psi_{1}(t)\right)=\lambda_{b}\left(A_{b}, \psi_{b}\right)=\lim _{t \rightarrow-\infty}\left(A_{2}(t), \psi_{2}(t)\right) .
$$


The elements $\left(\alpha_{i}, \phi_{i}\right)$ have exponentially decaying behavior, as in Proposition 3.12 .

We construct an approximate solution $x \#_{T}^{0} y=\left(\mathbb{A}_{1} \#_{T}^{0} \mathbb{A}_{2}, \Psi_{1} \#_{T}^{0} \Psi_{2}\right)$ of the form

$$
x \#_{T}^{0} y= \begin{cases}\lambda_{1}^{+}\left(\mathbb{A}_{1}^{2 T}, \Psi_{1}^{2 T}\right) & t \leq-1 \\ \lambda_{b}\left(A_{b}, \psi_{b}\right)+\rho^{-}(t)\left(\alpha_{1}(t+2 T), \phi_{1}(t+2 T)\right) & \\ +\rho^{+}(t)\left(\alpha_{2}(t-2 T), \phi_{2}(t-2 T)\right) & -1 \leq t \leq 1 \\ \lambda_{2}^{-}\left(\mathbb{A}_{2}^{-2 T}, \Psi_{2}^{-2 T}\right) & t \geq 1 .\end{cases}
$$

Here $\rho^{ \pm}(t)$ are smooth cutoff functions with bounded derivative, such that $\rho^{-}(t)$ is equal to one for $t \leq-1$ and to zero for $t \geq 0$ and $\rho^{+}(t)$ is equal to zero for $t \leq 0$ and to one for $t \geq 1$.

Lemma 4.10. The pre-gluing map $\#_{T}^{0}$ descends to a map at the level of gauge classes. Namely, it is well defined with respect to the choice of slices of the gauge action.

Proof. Consider a different choice of slices

$$
\mathcal{S}_{\tilde{\Gamma}_{a b}} \subset \mathcal{A}_{k, \delta}\left(O_{a}, O_{b}\right)
$$

determined by

$$
\tilde{\Gamma}_{a b} \in \mathcal{A}_{k, \delta}\left(\left(\tilde{A}_{a}, \tilde{\psi}_{a}\right),\left(\tilde{A}_{b}, \tilde{\psi}_{b}\right)\right)
$$

and

$$
\mathcal{S}_{\tilde{\Gamma}_{b c}} \subset \mathcal{A}_{k, \delta}\left(O_{b}, O_{c}\right)
$$

determined by

$$
\tilde{\Gamma}_{b c} \in \mathcal{A}_{k, \delta}\left(\left(\tilde{A}_{b}, \tilde{\psi}_{b}\right),\left(\tilde{A}_{c}, \tilde{\psi}_{c}\right)\right) .
$$

Suppose that $x$ and $y$ are now in balls of radius $r^{\prime} \leq r$ centered at $\tilde{\Gamma}_{a b}$ and $\tilde{\Gamma}_{b c}$ respectively. Then, according to Lemma 4.8 , there are gauge transformations $\tilde{\lambda}_{1}^{+}$and $\tilde{\lambda}_{2}^{-}$that conjugate $x$ and $y$ so that we get

$$
\tilde{\lambda}_{1}^{+} x \in W_{\tilde{\Gamma}_{a b}^{+}}^{+}, \quad \tilde{\lambda}_{2}^{-} y \in W_{\tilde{\Gamma}_{b c}^{-}}^{-} .
$$

Consider the pre-glued element of the form

$$
\tilde{x} \#_{T}^{0} \tilde{y}= \begin{cases}\tilde{\lambda}_{1}^{+}\left(\mathbb{A}_{1}^{2 T}, \Psi_{1}^{2 T}\right) & t \leq-1 \\ \tilde{\lambda}_{b}\left(\tilde{A}_{b}, \tilde{\psi}_{b}\right)+\rho^{-}(t)\left(\tilde{\alpha}_{1}(t+2 T), \tilde{\phi}_{1}(t+2 T)\right) & \\ +\rho^{+}(t)\left(\tilde{\alpha}_{2}(t-2 T), \tilde{\phi}_{2}(t-2 T)\right) & -1 \leq t \leq 1 \\ \tilde{\lambda}_{2}^{-}\left(\mathbb{A}_{2}^{-2 T}, \Psi_{2}^{-2 T}\right) & t \geq 1 .\end{cases}
$$


The temporal gauge representatives are defined up to an ambiguity given by the action gauge transformations that are constant in the $\mathbb{R}$ direction, that is by the action of $\mathcal{G}(Y)$. Thus, on the interval $[-1,1]$, the gauge transformations $\tilde{\lambda}_{i}^{ \pm} \circ \lambda_{i}^{ \pm-1}$ are constant with respect to $t$,

$$
\left.\tilde{\lambda}_{i}^{ \pm} \circ \lambda_{i}^{ \pm-1}\right|_{Y \times[-1,1]}=\tilde{\lambda}_{b} \lambda_{b}^{-1} .
$$

Thus $\tilde{\lambda}_{1}^{+} \circ \lambda_{1}^{+-1}$ and $\tilde{\lambda}_{2}^{-} \circ \lambda_{2}^{--1}$ define a gauge transformation $\lambda$ in $\mathcal{G}(Y \times \mathbb{R})$ (depending on $T$ ) such that

$$
\tilde{x} \#_{T}^{0} \tilde{y}=\lambda\left(x \#_{T}^{0} y\right)
$$

is satisfied. Thus, Lemma 4.7 and Lemma 4.8 imply that the pre-gluing map induces a well defined map $\#_{T}^{0}$ on compact subsets of broken trajectories,

$$
\#_{T}^{0}: K \subset \hat{\mathcal{M}}\left(O_{a}, O_{b}\right) \times_{O_{b}} \hat{\mathcal{M}}\left(O_{b}, O_{c}\right) \rightarrow \hat{\mathcal{B}}_{k, \delta}^{0}\left(O_{a}, O_{c}\right)
$$

The proof of Theorem 4.9 will consist of showing that the pre-glued approximate solution $x \#_{T}^{0} y$ can be perturbed to an actual solution of the flow equations by a small perturbation.

We need a preliminary discussion of some properties of the linearization of the Seiberg-Witten equations at the pre-glued solution.

Consider the weight function $e_{\delta^{T}}(t)=e^{\delta^{T}(t)}$, with $\delta^{T}(t)$ a function that satisfies $e_{\delta T}(t)=e^{\delta(t+T)}$ for $t<0$ and $e_{\delta^{T}}(t)=e^{\delta(t-T)}$ for $t>0$. Here $e_{\delta}(t)=e^{\delta(t)}$ is the usual weight function introduced in Proposition 2.9. We define the norm $L_{k, \delta(T)}^{2}$ as $\|f\|_{L_{k, \delta(T)}^{2}}=\left\|f e_{\delta^{T}}\right\|_{L_{k}^{2}}$. We can choose the weight function $e_{\delta^{T}}(t)$ as above, so that the weighted norms satisfy the estimates

$$
C_{1} e^{\delta T}\|f\|_{L_{k, \delta}^{2}} \leq\|f\|_{L_{k, \delta(T)}^{2}} \leq C_{2} e^{\delta T}\|f\|_{L_{k, \delta}^{2}}
$$

with constants $C_{1}$ and $C_{2}$ independent of $T$.

Suppose given classes $[x]=\left[\mathbb{A}_{1}, \Psi_{1}\right]$ and $[y]=\left[\mathbb{A}_{2}, \Psi_{2}\right]$ in $\mathcal{M}\left(O_{a}, O_{b}\right)$ and $\mathcal{M}\left(O_{b}, O_{c}\right)$ respectively. We can consider the operator

$$
\mathcal{L}_{x}(\alpha, \Phi)=\left\{\begin{array}{l}
d^{+} \alpha-\frac{1}{2} \operatorname{Im}\left(\Psi_{1} \cdot \bar{\Phi}\right)+\mathcal{D} P_{\left(\mathbb{A}_{1}, \Psi_{1}\right)}(\alpha, \Phi) \\
D_{\mathbb{A}_{1}} \Phi+\alpha \Psi_{1} \\
G_{\Gamma_{a b}}^{*}(\alpha, \Phi)
\end{array}\right.
$$


acting on the space of $L_{1, \delta}^{2}$-decaying 1-forms and spinor sections on $Y \times \mathbb{R}$. Analogously we have

$$
\mathcal{L}_{y}(\alpha, \Phi)=\left\{\begin{array}{l}
d^{+} \alpha-\frac{1}{2} \operatorname{Im}\left(\Psi_{2} \cdot \bar{\Phi}\right)+\mathcal{D} P_{\left(\mathbb{A}_{2}, \Psi_{2}\right)}(\alpha, \Phi) \\
D_{\mathbb{A}_{2}} \Phi+\alpha \Psi_{2} \\
G_{\Gamma_{b c}}^{*}(\alpha, \Phi)
\end{array}\right.
$$

acting on $L_{1, \delta}^{2} 1$-forms and spinor sections on $Y \times \mathbb{R}$. We also consider

$$
\mathcal{L}_{x \#_{T}^{0} y}(\alpha, \Phi)=\left\{\begin{array}{l}
d^{+} \alpha-\frac{1}{2} \operatorname{Im}\left(\Psi_{1} \#_{T}^{0} \Psi_{2} \cdot \bar{\Phi}\right)+\mathcal{D} P_{\left(\mathbb{A}_{1} \#_{T}^{0} \mathbb{A}_{2}, \Psi_{1} \#_{T}^{0} \Psi_{2}\right)}(\alpha, \Phi) \\
D_{\mathbb{A}_{1} \#_{T}^{0} \mathbb{A}_{2}} \Phi+\alpha \cdot\left(\Psi_{1} \#_{T}^{0} \Psi_{2}\right) \\
G_{\Gamma_{a c}}^{*}(\alpha, \Phi)
\end{array}\right.
$$

acting on $L_{1, \delta(T)}^{2} 1$-forms and spinor sections on $Y \times \mathbb{R}$. Moreover, if $\left(A_{b}, \psi_{b}\right)$ is a representative of a point on $O_{b}$, with $O_{b}$ a free $U(1)$-orbit, consider the operator

$$
\mathcal{L}_{b}(\alpha, \Phi)=\left\{\begin{array}{l}
d^{+} \alpha-\frac{1}{2} \operatorname{Im}\left(\psi_{b} \cdot \bar{\Phi}\right) \\
D_{A_{b}} \Phi+\alpha \psi_{b} \\
G_{\left(A_{b}, \psi_{b}\right)}^{*}(\alpha, \Phi)
\end{array}\right.
$$

acting on $L_{1}^{2} 1$-forms and spinor sections on $Y \times \mathbb{R}$. If $O_{b} \equiv b$ is fixed by the $U(1)$-action, consider the operator

$$
\mathcal{L}_{b}(\alpha, \Phi)=\left\{\begin{array}{l}
d^{+} \alpha \\
D_{A_{b}} \Phi \\
G_{\left(A_{b}, \psi_{b}\right)}^{*}(\alpha, \Phi)
\end{array}\right.
$$

acting on $L_{1}^{2} 1$-forms and spinor sections on $Y \times \mathbb{R}$.

The central technique in the gluing construction is the study of the behavior of the eigenspaces of small eigenvalues of the Laplacians $\mathcal{L}_{x \#_{T}^{*} y}^{*} \mathcal{L}_{x \#_{T}^{0} y}$ and $\mathcal{L}_{x \#_{T}^{0} y} \mathcal{L}_{x \#_{T}^{0} y}^{*}$ of the linearization of the Seiberg-Witten equations at the approximate solutions. The general philosophy can be summarized as follows: we are trying to paste together solutions of a system of non-linear elliptic equations. If the equations were linear, we would simply encounter the obstruction to inverting the operator $\mathcal{L}_{x \#_{T}^{0} y}^{*}$. This obstruction is represented by $\operatorname{Coker}\left(\mathcal{L}_{x \#_{T}^{0} y}\right)$. However, the equations being non-linear, the presence of eigenvectors with small eigenvalues of $\mathcal{L}_{x \#_{T}^{0} y} \mathcal{L}_{x \#_{T}^{0} y}^{*}$ also represents obstructions. (cf. the discussion of this phenomenon in [60], pg. 
169). For this reason, we introduce the notation "approximate cokernel", $\operatorname{ApprCoker}\left(\mathcal{L}_{x \#_{T}^{0} y}\right)$ (cf. Definition 4.16) to denote this eigenspace of small eigenvalues. The notation may be slightly confusing, as it may suggest a space of approximate eigenvectors, whereas it simply denotes the eigenspace of small eigenvalues, as explained in Definition 4.16. We trust that the reader will not be confused by our, perhaps unorthodox, choice of notation.

So the central technical issue in the gluing construction becomes relating the small eigenvalues eigenspace of the Laplacian $\mathcal{L}_{x \#_{T}^{0} y} \mathcal{L}_{x \#_{T}^{0} y}^{*}$ (we call this space ApprCoker $\left(\mathcal{L}_{x \#_{T}^{0} y}\right)$ ) to the kernels of the Laplacians $\mathcal{L}_{x} \mathcal{L}_{x}^{*}, \mathcal{L}_{y} \mathcal{L}_{y}^{*}$, and $\mathcal{L}_{b} \mathcal{L}_{b}^{*}$, and similarly for the other Laplacians $\mathcal{L}_{x}^{*} \mathcal{L}_{x}$ and $\mathcal{L}_{y}^{*} \mathcal{L}_{y}$ and the small eigenvalues eigenspace of $\mathcal{L}_{x \#_{T}^{0} y}^{*} \mathcal{L}_{x \#_{T}^{0} y}$ (which we call $\operatorname{Appr} \operatorname{Ker}\left(\mathcal{L}_{x \#_{T}^{0} y}\right)$ ).

First of all we can identify explicitly the kernel and cokernel of the operator $\mathcal{L}_{b}$ as in (40) and (41).

Lemma 4.11. The operator $\mathcal{L}_{b}$ defined as in (41), that is, for $O_{b} \equiv b$ the fixed point of the $U(1)$-action, has trivial $L_{1, \delta^{-}}^{2}$-kernel and trivial $L_{0, \delta^{-}}^{2}$ cokernel. The operator $\mathcal{L}_{b}$ defined as in (40), with $O_{b}$ a free $U(1)$-orbit, also has trivial kernel in $L_{1, \delta}^{2}$, and trivial cokernel in $L_{0, \delta}^{2}$.

Proof. We prove the case of (41), where the critical orbit $O_{b} \equiv b$ is the unique fixed point of the $U(1)$ action $b=\theta$. The irreducible case, with a free $U(1)$ orbit $O_{b}$ follows by an analogous argument.

Let $(\alpha, \Phi)$ satisfy

$$
\left\{\begin{array}{l}
d^{+} \alpha=0 \\
D_{A_{b}} \Phi=0 \\
G_{A_{b}, 0}^{*}(\alpha, \Phi)=0,
\end{array}\right.
$$

where the operator $G_{A_{b}, 0}^{*}$ is the adjoint of the infinitesimal gauge group action with respect to the $L_{0, \delta}^{2}$ inner product. In this case, this is simply given by the operator $e_{\delta} d^{*} e_{-\delta}$. The Dirac equation $D_{A_{b}} \Phi=0$ can be rewritten in terms of the 3-dimensional Dirac operator, $\left(\partial_{t}+\partial_{A_{b}}\right) \Phi=0$. Upon expanding the spinor on a basis of eigenvectors for the 3-dimensional Dirac operator $\partial_{A_{b}}$, we obtain $\Phi=\sum a_{k}(t) \phi_{k}$ and the equation has solutions $\Phi(t)=\sum_{k} a_{k}(0) e^{\lambda_{k} t} \phi_{k}$. Since none of these solutions is in $L_{1, \delta}^{2}$, we have obtained $\Phi \equiv 0$. Now we consider the curvature equation. This gives $d^{+} \alpha=0$ and $e_{\delta} d^{*} e_{-\delta} \alpha=0$. These two conditions imply that $e_{-\delta / 2} \alpha$ is a harmonic representative in $H_{\delta / 2}^{2+}(Y \times \mathbb{R})$, with respect to the Laplacian $\Delta_{\delta / 2}=e_{\delta / 2} \Delta e_{-\delta / 2}$. For a rational homology sphere $Y$ (the only case where we have to consider the presence of the reducible point $\theta$ ), this is necessarily 
trivial. The argument for the cokernel is similar. Consider an element in the cokernel, namely, suppose given a triple $(\beta, f, \xi)$ of a self-dual 2-form, a 0 -form, and a spinor section of the bundle of negative spinors over $Y \times \mathbb{R}$, such that we have

$$
\left\langle\beta, d^{+} \alpha\right\rangle+\left\langle f, e_{\delta} d^{*} e_{-\delta} \alpha\right\rangle+\left\langle\xi, D_{A_{b}} \Phi\right\rangle=0
$$

in the $L^{2}$ pairing. We then have $D_{A_{b}} \xi=0$ which implies $\xi=0$ by the previous argument, and $d^{*} \omega=e_{-\delta} d e_{\delta} f$. This equation implies $d^{*} \omega=0$ and $e_{-\delta} d e_{\delta} f=0$. The first equation then implies that $\omega$ is a harmonic representative in $H^{2+}(Y \times \mathbb{R})$. Again, this is trivial since the only case where the reducible $\theta$ appears is when $Y$ is a rational homology sphere. The remaining equation gives $f=c e_{-\delta}$. The condition $f\left(x_{0}\right)=0$ at the base point $x_{0}=\left(y_{0}, t_{0}\right)$ implies $c=0$, hence $f \equiv 0$.

The following Lemma, together with Lemma 4.13 and Lemma 4.15, contains the key argument that will be used in Proposition 4.17 to relate the spaces ApprCoker $\left(\mathcal{L}_{x \#_{T}^{0} y}\right)$ and $\operatorname{ApprKer}\left(\mathcal{L}_{x \#_{T}^{0} y}\right)$ of eigenvectors of small eigenvalues to the kernels and cokernels of $\mathcal{L}_{x}$ and $\mathcal{L}_{y}$ and in Corollary 4.18 for the formula on the splitting of the index.

Lemma 4.12. Let $x$ and $y$ be elements in $\mathcal{M}\left(O_{a}, O_{b}\right)$ and $\mathcal{M}\left(O_{b}, O_{c}\right)$ respectively. Suppose given a sequence $\xi_{k}$ of $L_{1, \delta}^{2} 1$-forms and spinor sections on $Y \times \mathbb{R}$. Assume these elements live in the tangent space, in the $L_{1, \delta}^{2}-$ norm, to the fiber $\left(e_{a}^{+}\right)^{-1}\left(x_{a}\right)$ of the endpoint map

$$
e_{a}^{+}: \mathcal{S}_{\Gamma_{a c}} \rightarrow O_{a},
$$

with $x_{a}$ the asymptotic value at $t \rightarrow-\infty$ of $x \in \mathcal{M}\left(O_{a}, O_{b}\right)$, and for a given choice of slices $\mathcal{S}_{\Gamma_{a b}}$ and $\mathcal{S}_{\Gamma_{b c}}$ as in (32) and (33), and

$$
\mathcal{S}_{\Gamma_{a c}} \subset \mathcal{A}_{1, \delta}\left(O_{a}, O_{c}\right) .
$$

Suppose given a corresponding sequence of gluing parameters $T_{k}$ with $T_{k} \rightarrow \infty$. We assume that $\xi_{k}$ and $T_{k}$ satisfy $e^{\delta T_{k}}\left\|\xi_{k}\right\|_{L_{1, \delta}^{2}}=1$ and $\left\|\mathcal{L}_{x \#_{T_{k}}^{0} y} \xi_{k}\right\|_{L_{0, \delta\left(T_{k}\right)}^{2}} \rightarrow 0$, with $\mathcal{L}_{x \#_{T_{k}}^{0} y}$ as in (39). We have $L_{1}^{2}$ convergence $\left.e^{\delta T_{k}} \xi_{k}\right|_{Y \times\left[-T_{k}, T_{k}\right]} \rightarrow 0$. Then there exist $L_{1, \delta}^{2}$-elements $u$ and $v$ in $\operatorname{Ker}\left(\mathcal{L}_{x}\right)$ and $\operatorname{Ker}\left(\mathcal{L}_{y}\right)$ respectively, with $\mathcal{L}_{x}$ and $\mathcal{L}_{y}$ as in (37) and (38), such that we have convergence

$$
\left\|\rho_{1-T_{k}}^{-} \xi_{k}^{-T_{k}}-u\right\|_{L_{1, \delta}^{2}} \rightarrow 0
$$


and

$$
\left\|\rho_{-1+T_{k}}^{+} \xi_{k}^{T_{k}}-v\right\|_{L_{1, \delta}^{2}} \rightarrow 0 .
$$

Here we have $\rho_{-1+T_{k}}^{+}(t)=\rho^{+}\left(t-1+T_{k}\right)$ and $\rho_{1-T_{k}}^{-}(t)=\rho^{-}\left(t+1-T_{k}\right)$.

Proof. We first want to prove that the supports of the $\xi_{k}$ become more and more concentrated at the asymptotic ends as $k \rightarrow \infty$. Consider the operator $\mathcal{L}_{b}$ as in (40) or (41). If $\zeta: Y \times \mathbb{R} \rightarrow[0,1]$ is a smooth function which is equal to 1 on $Y \times[-1 / 2,1 / 2]$ and equal to zero outside $Y \times(-1,1)$. Moreover suppose that, for any fixed $t$, the function $\zeta$ is constant on $Y \times\{t\}$. Let $\zeta_{k}(t)=\zeta\left(\frac{t}{T_{k}}\right)$. Then, using the pointwise estimate $\left|\zeta_{k}\right| \leq 1$, we have

$$
\begin{aligned}
& \left\|\mathcal{L}_{b} \zeta_{k} e^{\delta T_{k}} \xi_{k}\right\|_{L_{0, \delta}^{2}} \\
& \leq\left\|\zeta_{k}^{\prime} e^{\delta T_{k}} \xi_{k}\right\|_{L_{0, \delta}^{2}}+\left\|\zeta_{k} e^{\delta T_{k}} \mathcal{L}_{b} \xi_{k}\right\|_{L_{0, \delta}^{2}} \\
& \leq \frac{1}{T_{k}} \max \left|\zeta^{\prime}\right|+\left\|\zeta_{k}\left(\mathcal{L}_{x \#_{T_{k}} y}-\mathcal{L}_{b}\right) e^{\delta T_{k}} \xi_{k}\right\|_{L_{0, \delta}^{2}}+\left\|e^{\delta T_{k}} \mathcal{L}_{x \#_{T_{k}} y} \xi_{k}\right\|_{L_{0, \delta}^{2}} \\
& \leq \frac{1}{T_{k}} \max \left|\zeta^{\prime}\right|+\sup _{t \in\left[-T_{k}, T_{k}\right]}\left\|\mathcal{L}_{x \#_{T_{k}}^{0} y}-\mathcal{L}_{b}\right\|\left\|e^{\delta T_{k}} \xi_{k}\right\|_{L_{1, \delta}^{2}}+\left\|\mathcal{L}_{x \#_{T_{k}}^{0} y} \xi_{k}\right\|_{L_{0, \delta\left(T_{k}\right)}^{2}} .
\end{aligned}
$$

The first and last term tend to zero as $k \rightarrow \infty$. The remaining term

$$
\sup _{t \in\left[-T_{k}, T_{k}\right]}\left\|\mathcal{L}_{x \#_{T_{k}}^{0} y}-\mathcal{L}_{b}\right\|\left\|e^{\delta T_{k}} \xi_{k}\right\|_{L_{1, \delta}^{2}}
$$

is bounded by

$$
\sup _{t \in[-1,1]}\left\|\mathcal{L}_{x \#_{T_{k}}^{0} y}-\mathcal{L}_{b}\right\|+\sup _{t \in\left[-T_{k},-1\right]}\left\|\mathcal{L}_{x^{2 T_{k}}}-\mathcal{L}_{b}\right\|+\sup _{t \in\left[1, T_{k}\right]}\left\|\mathcal{L}_{y^{-2 T_{k}}}-\mathcal{L}_{b}\right\| .
$$

All these terms tend to zero because of the exponential decay to the critical point $b$ of the trajectories $\left(A_{1}(t), \psi_{1}(t)\right)$ and $\left(A_{2}(t), \psi_{2}(t)\right)$, and because of condition (5) of Definition 2.10. Thus, we have $\left\|\mathcal{L}_{b} \zeta_{k} e^{\delta T_{k}} \xi_{k}\right\|_{L_{0, \delta}^{2}} \rightarrow 0$ as $k \rightarrow \infty$.

Lemma 4.11 shows that the condition $\left\|\mathcal{L}_{b} \zeta_{k} e^{\delta T_{k}} \xi_{k}\right\|_{L_{0, \delta}^{2}} \rightarrow 0$ implies the convergence $\zeta_{k} e^{\delta T_{k}} \xi_{k} \rightarrow 0$ in the $L_{1, \delta}^{2}$-norm, and therefore we have

$$
\left\|e^{\delta T_{k}} \xi_{k}\right\|_{L_{1}^{2}\left(Y \times\left[-T_{k}, T_{k}\right]\right)} \rightarrow 0 .
$$

In other words, the $\xi_{k}$ get localized at the asymptotic ends exponentially fast. 
This result allows us to rephrase the convergence condition $\left\|\mathcal{L}_{x \#_{T_{k}}^{0}} \xi_{k}\right\| \rightarrow 0$ in terms of the Fredholm operators $\mathcal{L}_{x}$ and $\mathcal{L}_{y}$ :

$$
\begin{aligned}
& \left\|\mathcal{L}_{x}\left(\rho_{1-T_{k}}^{-} \xi_{k}^{-T_{k}}\right)\right\|_{L_{0, \delta}^{2}} \\
& \leq\left\|\rho_{1-T_{k}}^{-} \xi_{k}^{-T_{k}}\right\|_{L_{0, \delta}^{2}}+\left\|\rho^{-} \mathcal{L}_{x \#_{T_{k}}^{0} y} \xi_{k}\right\|_{L_{0, \delta\left(T_{k}\right)}^{2}} \\
& \leq C\left\|\rho_{1}^{-} e^{\delta T_{k}} \xi_{k}\right\|_{L_{0, \delta}^{2}}+\left\|\rho^{-} \mathcal{L}_{x \#_{T_{k}}^{0} y} \xi_{k}\right\|_{L_{0, \delta\left(T_{k}\right)}^{2}} \rightarrow \|{ } \rightarrow 0, \\
& \leq C\left\|e^{\delta T_{k}} \xi_{k}\right\|_{L_{1}^{2}(Y \times[-1,1])}+\left\|\mathcal{L}_{x \#_{T_{k}}^{0} y} \xi_{k}\right\|_{L_{0, \delta\left(T_{k}\right)}^{2}} \rightarrow 0,
\end{aligned}
$$

where $\rho_{1-T_{k}}^{-}(t)=\rho\left(t+1-T_{k}\right)$ and $\xi_{k}^{-T_{k}}(t)=\xi_{k}\left(t-T_{k}\right)$.

The linearization $\mathcal{L}_{x}$ is a Fredholm operator. The sequence $\rho_{1-T_{k}}^{-} \xi_{k}^{-T_{k}}$ is uniformly bounded in the $L_{1, \delta}^{2}$ norm: in fact, by the estimate (36) we have

$$
\left\|\rho_{1-T_{k}}^{-} \xi_{k}^{-T_{k}}\right\|_{L_{1, \delta}^{2}} \leq C\left\|\rho_{1}^{-} e^{\delta T_{k}} \xi_{k}\right\|_{L_{1, \delta}^{2}} \leq C .
$$

This implies the existence of an $L_{1, \delta}^{2}$-element $u$ in $\operatorname{Ker}\left(\mathcal{L}_{x}\right)$ such that, upon passing to a subsequence, we obtain the $L_{1, \delta}^{2}$ convergence

$$
\left\|\rho_{1-T_{k^{\prime}}}^{-} \xi_{k^{\prime}}^{-T_{k^{\prime}}}-u\right\|_{L_{1, \delta}^{2}} \rightarrow 0
$$

This implies the convergence

$$
\left\|\rho_{1}^{-} e^{\delta T_{k^{\prime}}} \xi_{k^{\prime}}-\rho^{-} e^{\delta T_{k^{\prime}}} u^{T_{k^{\prime}}}\right\|_{L_{1, \delta}^{2}} \rightarrow 0 .
$$

Similarly we obtain an $L_{1, \delta}^{2}$-element $v$ in $\operatorname{Ker}\left(\mathcal{L}_{y}\right)$ and a subsequence such that

$$
\left\|\rho_{-1+T_{k^{\prime}}}^{+} \xi_{k^{\prime}}^{T_{k^{\prime}}}-v\right\|_{L_{1, \delta}^{2}} \rightarrow 0
$$

and

$$
\left\|\rho_{-1}^{+} e^{\delta T_{k^{\prime}}} \xi_{k^{\prime}}-\rho^{+} e^{\delta T_{k^{\prime}}} v^{-T_{k^{\prime}}}\right\|_{L_{1, \delta}^{2}} \rightarrow 0 .
$$

Here we have $\rho_{-1}^{+}(t)=\rho^{+}(t-1)$ and $\rho_{1}^{-}(t)=\rho^{-}(t+1)$.

Thus, by Lemma 4.12 we have identified the eigenspace of small eigenvalues of $\mathcal{L}_{x \#_{T}^{0} y}^{*} \mathcal{L}_{x \#_{T}^{0} y}$ with a subspace of $\operatorname{Ker}\left(\mathcal{L}_{x}\right) \times \operatorname{Ker}\left(\mathcal{L}_{y}\right)$. Lemma 4.15 
will prove the reverse inclusion. The next Lemma proves a stronger result, namely that the operator $\mathcal{L}_{x \#_{T}^{0} y}^{*} \mathcal{L}_{x \#_{T}^{0} y}$ is uniformly invertible, in the rescaled norm $L_{1, \delta(T)}^{2}$, on the complement of the eigenspace of small eigenvalues. This is the essential result on the eigenvalue splitting which we are going to use in Section 6, in Lemma 6.13.

Let us introduce the following notation. Let $F_{\#_{T}}$ be the linearization of the pre-gluing map (35). Thus, $F_{\#_{T}}$ is a map

$$
\left.F_{\# T}\right|_{(x, y)}: \operatorname{Ker}\left(\mathcal{L}_{x}\right) \times \operatorname{Ker}\left(\mathcal{L}_{y}\right) \rightarrow \mathcal{T}_{1}\left(\left(e_{a}^{+}\right)^{-1}\left(x_{a}\right)\right) \subset \mathcal{T}_{1}\left(\mathcal{B}_{k, \delta}^{0}\left(O_{a}, O_{b}\right)\right)
$$

Here $x_{a}$ is the asymptotic value as $t \rightarrow-\infty$ of $x$ and $e_{a}^{+}$is the endpoint map $e_{a}^{+}: \mathcal{S}_{\Gamma_{a c}} \rightarrow O_{a}$. Since the pre-glued solution (35) involves translations, the map $F_{\#_{T}}$ in (44) has operator norm bounded by $C_{T} \sim C e^{-\delta T}$, namely, we have

$$
\left\|\left.F_{\#_{T}}\right|_{(x, y)}(u, v)\right\|_{1, \delta} \leq C e^{-\delta T}\|(u, v)\|_{1, \delta} .
$$

We may also consider the same map acting as

$$
\begin{aligned}
\left.F_{\#_{T}}\right|_{(x, y)}: \operatorname{Ker}\left(\mathcal{L}_{x}\right) \times & \operatorname{Ker}\left(\mathcal{L}_{y}\right) \\
& \rightarrow \mathcal{T}_{1, T}\left(\left(e_{a}^{+}\right)^{-1}\left(x_{a}\right)\right) \subset \mathcal{T}_{1, T}\left(\mathcal{B}_{k, \delta(T)}^{0}\left(O_{a}, O_{b}\right)\right),
\end{aligned}
$$

with $\mathcal{T}_{1, T}$ the virtual tangent space in the $L_{1, \delta(T)}^{2}$ norm. In this case $F_{\#_{T}}$ is bounded in the operator norm uniformly in $T$, given the estimate (36) for the rescaled norms.

Lemma 4.13. There exist $T_{0}$ such that

$$
\left\|\mathcal{L}_{x \#_{T}^{0} y} \xi\right\|_{L_{0, \delta(T)}^{2}} \geq C_{T}\|\xi\|_{L_{1, \delta}^{2}}
$$

for all $T \geq T_{0}$ and for all $\xi$ in the orthogonal complement

$$
\mathcal{T}^{\perp}=\left(F_{\# T}\left(\operatorname{Ker}\left(\mathcal{L}_{x}\right) \times \operatorname{Ker}\left(\mathcal{L}_{y}\right)\right)\right)^{\perp}
$$

in the space of $L_{1, \delta}^{2}$-connections and sections. With this choice of norms, the constant $C_{T}$ grows like $C e^{\delta T}$. If we consider the rescaled norms $L_{\ell, \delta(T)}^{2}$ or the original norms $L_{\ell, \delta}^{2}$ on both the source and target space, the constant is bounded uniformly in $T$, given the estimate (36) for the rescaled norms. 
Proof. Suppose there is a sequence of parameters $T_{k}$ and elements $\xi_{k}$ in the orthogonal complement

$$
\xi_{k} \in\left(F_{\# T_{k}}\left(\operatorname{Ker}\left(\mathcal{L}_{x}\right) \times \operatorname{Ker}\left(\mathcal{L}_{y}\right)\right)\right)^{\perp}
$$

in the space of $L_{1, \delta^{-}}^{2}$-connections and sections, such that $e^{\delta T_{k}}\left\|\xi_{k}\right\|_{L_{1, \delta}^{2}}=1$ and $\left\|\mathcal{L}_{x \#_{T_{k}}^{0} y} \xi_{k}\right\|_{L_{0, \delta\left(T_{k}\right)}^{2}} \rightarrow 0$. We use the estimates of Lemma 4.12 to derive a contradiction with the assumption $\xi_{k} \in \mathcal{T}^{\perp}$. We have

$$
1=\lim _{k} e^{2 \delta T_{k}}\left\|\xi_{k}\right\|_{L_{1, \delta}^{2}}^{2}=\lim _{k}\left\langle\rho_{1}^{-} e^{\delta T_{k}} \xi_{k}, e^{\delta T_{k}} \xi_{k}\right\rangle_{L_{1, \delta}^{2}}+\left\langle\rho_{-1}^{+} e^{\delta T_{k}} \xi_{k}, e^{\delta T_{k}} \xi_{k}\right\rangle_{L_{1, \delta}^{2}},
$$

since the remaining term satisfies

$$
\lim _{k}\left\langle\left(1-\rho_{1}^{-}-\rho_{-1}^{+}\right) e^{\delta T_{k}} \xi_{k}, e^{\delta T_{k}} \xi_{k}\right\rangle=0 .
$$

This follows from Lemma 4.12, since $\left(1-\rho_{1}^{-}-\rho_{-1}^{+}\right)$is supported in $[-2,2]$, and we are considering the case where $\operatorname{Ker}\left(\mathcal{L}_{b}\right)=0$ in $L_{1, \delta}^{2}$.

Moreover, we know from Lemma 4.12 that, upon passing to a subsequence we have convergence as in (42) and (43). This implies that, upon passing to a subsequence, we obtain

$$
\lim _{k}\left\langle\rho_{1}^{-} e^{\delta T_{k}} \xi_{k}, e^{\delta T_{k}} \xi_{k}\right\rangle_{L_{1, \delta}^{2}}=\lim _{k} e^{2 \delta T_{k}}\left\langle\rho^{-} u^{T_{k}}, \xi_{k}\right\rangle_{L_{1, \delta}^{2}},
$$

and correspondingly

$$
\lim _{k}\left\langle\rho_{-1}^{+} e^{\delta T_{k}} \xi_{k}, e^{\delta T_{k}} \xi_{k}\right\rangle_{L_{1, \delta}^{2}}=\lim _{k} e^{2 \delta T_{k}}\left\langle\rho^{+} v^{-T_{k}}, \xi_{k}\right\rangle_{L_{1, \delta}^{2}} .
$$

The sum of these two terms therefore gives

$$
\lim _{k} e^{2 \delta T_{k}}\left\langle F_{\#_{T_{k}}}(u, v), \xi_{k}\right\rangle_{L_{1, \delta}^{2}} .
$$

But this term is zero since we are assuming that the elements $\xi_{k}$ are in the orthogonal complement

$$
\xi_{k} \in\left(F_{\#_{T_{k}}}\left(\operatorname{Ker}\left(\mathcal{L}_{x}\right) \times \operatorname{Ker}\left(\mathcal{L}_{y}\right)\right)\right)^{\perp} .
$$


Remark 4.14. The results of Lemma 4.12 and Lemma 4.13 can be reformulated and proved, with minor modifications, for the operator $\mathcal{L}_{x \#_{T}^{0} y}^{*}$ and elements

$$
(u, v) \in \operatorname{Ker}\left(\mathcal{L}_{x}^{*}\right) \times \operatorname{Ker}\left(\mathcal{L}_{y}^{*}\right) .
$$

Namely, suppose given a sequence $\xi_{k}$ in $\mathcal{T}_{0}\left(\mathcal{S}_{\Gamma_{a c}}\right)$, bounded uniformly in the $L_{0, \delta\left(T_{k}\right)}^{2}$-norms, such that $\mathcal{L}_{x \#_{T_{k}}^{0} y}^{*} \xi_{k} \rightarrow 0$ as $k \rightarrow \infty$. Then we have convergence $\left\|\mathcal{L}_{b}^{*} \zeta_{k} \xi_{k}\right\|_{L_{1, \delta}^{2}} \rightarrow 0$, which implies $L_{0, \delta}^{2}$-convergence of the elements $\zeta_{k} \xi_{k}$ to zero. Moreover, there exist elements $u \in \operatorname{Coker}\left(\mathcal{L}_{x}\right)$ and $v \in \operatorname{Coker}\left(\mathcal{L}_{y}\right)$ satisfying

$$
\left\|\rho_{1-T_{k}}^{-} \xi_{k}^{-T_{k}}-u\right\|_{0, \delta} \rightarrow 0
$$

and

$$
\left\|\rho_{-1+T_{k}}^{+} \xi_{k}^{T_{k}}-v\right\|_{0, \delta} \rightarrow 0 .
$$

Thus, for all $T \geq T_{0}$, there is an estimate

$$
\left\|\mathcal{L}_{x \#_{T}^{0} y}^{*} \xi\right\|_{1, \delta} \geq C\|\xi\|_{0, \delta}
$$

for all $\xi$ in

$$
\left(F_{\# T}\left(\operatorname{Coker}\left(\mathcal{L}_{x}\right) \times \operatorname{Coker}\left(\mathcal{L}_{y}\right)\right)\right)^{\perp} \subset \mathcal{T}_{0}\left(\mathcal{S}_{\Gamma_{a c}}\right) .
$$

The constant $C$ is independent of $T \geq T_{0}$.

Thus, in Lemma 4.13 and Remark 4.14, we have obtained invertibility of the linearization of the pre-glued solution on the orthogonal complement of the linearization of the pre-gluing map. The norm of the inverse operator grows exponentially with the gluing parameter $T$. This is an effect of using the weighted Sobolev norms that are not translation invariant. If we use the rescaled norm $\delta(T)$, or the original $\delta$-norm on both the source and target space, we obtain uniform invertibility, with the norm of the inverse operator uniformly bounded in $T$.

Consider the two Laplacians of the gluing construction, namely, the selfadjoint operators

$$
H_{x \#_{T}^{0} y}^{0}=\mathcal{L}_{x \#_{T}^{0} y} \mathcal{L}_{x \#_{T}^{0} y}^{*}
$$

and

$$
H_{x \#_{T}^{0} y}^{1}=\mathcal{L}_{x \#_{T}^{0} y}^{*} \mathcal{L}_{x \#_{T}^{0} y}
$$

acting on $L_{0, \delta(T)}^{2}$ (or $L_{0, \delta}^{2}$ ) and on $L_{1, \delta(T)}^{2}$ (or $L_{1, \delta}^{2}$ ), respectively. 
Lemma 4.15. The operator $H_{x \#_{T}^{0} y}^{1}$ has at least

$$
\operatorname{dim} \operatorname{Ker}\left(\mathcal{L}_{x}\right)+\operatorname{dim} \operatorname{Ker}\left(\mathcal{L}_{y}\right)
$$

independent eigenvectors with small eigenvalues $\mu_{T}$ satisfying $\mu_{T} \rightarrow 0$ as $T \rightarrow \infty$. All these eigenvectors have fast decaying eigenvalues, namely $\mu_{T}$ satisfying $\mu_{T} \leq C e^{-\delta T}$. The operator $H_{x \#_{T}^{0} y}^{0}$ has at least

$$
\operatorname{dim} \operatorname{Coker}\left(\mathcal{L}_{x}\right)+\operatorname{dim} \operatorname{Coker}\left(\mathcal{L}_{y}\right)
$$

independent eigenvectors with small eigenvalues, namely with eigenvalues $\mu_{T}$ satisfying the property that $\mu_{T} \rightarrow 0$ as $T \rightarrow \infty$. Again, these have fast decaying eigenvalues, namely with $\mu_{T}$ satisfying $\mu_{T} \leq C e^{-\delta T}$.

Proof. Let us prove the case of $H_{x \#_{T}^{0} y}^{1}$ first. Suppose given an element $u$ in the $L_{1, \delta}^{2}$-kernel $\operatorname{Ker}\left(\mathcal{L}_{x}\right)$. Consider the element $\xi_{T}=\rho^{-} u^{T}$. We obtain the following estimate

$$
\left\|\mathcal{L}_{x \#_{T}^{0} y}\left(\xi_{T}\right)\right\|_{L_{0, \delta(T)}^{2}} \leq\left\|\mathcal{L}_{x} u\right\|_{L_{0, \delta}^{2}}+\left\|\mathcal{L}_{x \#_{T}^{0} y} e^{\delta T} \xi_{T}\right\|_{L_{1, \delta}^{2}(Y \times[-1,1])} .
$$

The first term on the right vanishes, and the second term can be estimated as

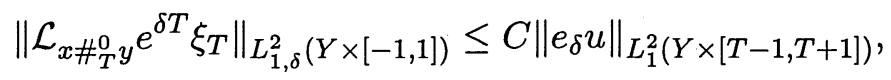

by the effect of time translations on the norms. This last term is certainly bounded by

$$
C \cdot\left\|\xi_{T}\right\|_{L_{1, \delta}^{2}}
$$

Thus, using the relation (36), we obtain

$$
\left\|\mathcal{L}_{x \#_{T}^{0} y}\left(\xi_{T}\right)\right\|_{L_{0, \delta}^{2}} \leq C e^{-\delta T}\left\|\xi_{T}\right\|_{L_{1, \delta}^{2}} .
$$

The same argument can be repeated starting with elements $v \in \operatorname{Ker}\left(\mathcal{L}_{y}\right)$ and constructing $\xi_{T}=\rho^{+} v^{-T}$. Notice moreover that if $u$ and $w$ are two independent vectors in $\operatorname{Ker}\left(\mathcal{L}_{x}\right)$, then the corresponding elements $\xi_{T}^{u}=\rho^{-} u^{T}$ and $\xi_{T}^{w}=\rho^{-} w^{T}$ are independent vectors in ApprCoker $\left(\mathcal{L}_{x \#_{T}^{0} y}\right)$, since we have

$$
\left\langle\xi_{T}^{u}, \xi_{T}^{w}\right\rangle_{L_{1, \delta(T)}^{2}} \rightarrow\langle u, w\rangle_{L_{1, \delta}^{2}}
$$

This proves the statement for $H_{x \#_{T}^{0} y}^{1}$. The argument for $H_{x \#_{T}^{0} y}^{0}$ is analogous.

Thus we can give the following definition. 
Definition 4.16. We define the approximate kernel and cokernel

$$
\operatorname{ApprKer}\left(\mathcal{L}_{x \#_{T}^{0} y}\right) \quad \operatorname{ApprCoker}\left(\mathcal{L}_{x \#_{T}^{0} y}\right)
$$

of the linearization at the pre-glued solution as the span of the eigenvectors of $H_{x \#_{T}^{1} y}^{1}$ and $H_{x \#_{T}^{0} y}^{0}$ respectively, with small eigenvalues, namely with eigenvalues $\mu_{T}$ satisfying $\mu_{T} \rightarrow 0$ as $T \rightarrow \infty$.

Lemma 4.12 and Lemma 4.13, together with Remark 4.14, and Lemma 4.15 give the following result.

Proposition 4.17. We have isomorphisms

$$
\operatorname{ApprKer}\left(\mathcal{L}_{x \#_{T}^{0} y}\right) \stackrel{\cong}{\rightrightarrows} \operatorname{Ker}\left(\mathcal{L}_{x}\right) \times \operatorname{Ker}\left(\mathcal{L}_{y}\right)
$$

and

$$
\operatorname{ApprCoker}\left(\mathcal{L}_{x \#{ }_{T}^{0} y}\right) \stackrel{\cong}{\operatorname{Coker}}\left(\mathcal{L}_{x}\right) \times \operatorname{Coker}\left(\mathcal{L}_{y}\right),
$$

given by the projections. Moreover, we obtain constraints on the dimensions of the actual kernel and cokernel of the linearization at the pre-glued solution:

$$
\operatorname{dim} \operatorname{Ker}\left(\mathcal{L}_{x \#_{T}^{0} y}\right) \leq \operatorname{dim} \operatorname{Ker}\left(\mathcal{L}_{x}\right)+\operatorname{dim} \operatorname{Ker}\left(\mathcal{L}_{y}\right)
$$

and

$$
\operatorname{dim} \operatorname{Coker}\left(\mathcal{L}_{x \#_{T}^{0} y}\right) \leq \operatorname{dim} \operatorname{Coker}\left(\mathcal{L}_{x}\right)+\operatorname{dim} \operatorname{Coker}\left(\mathcal{L}_{y}\right) .
$$

Proof. We have proved in Lemma 4.12 and Lemma 4.13 that

$$
\operatorname{dim} \operatorname{ApprKer}\left(\mathcal{L}_{x \#_{T}^{0} y}\right) \leq \operatorname{dim} \operatorname{Ker}\left(\mathcal{L}_{x}\right)+\operatorname{dim} \operatorname{Ker}\left(\mathcal{L}_{y}\right),
$$

and in Lemma 4.15 we have proved the reverse estimate, namely that

$$
\operatorname{dim} \operatorname{ApprKer}\left(\mathcal{L}_{x \#_{T}^{0} y}\right) \geq \operatorname{dim} \operatorname{Ker}\left(\mathcal{L}_{x}\right)+\operatorname{dim} \operatorname{Ker}\left(\mathcal{L}_{y}\right) .
$$

This, together with the result of Lemma 4.13 shows that the linearization of the gluing map $F_{\#}$ gives the isomorphism of the Proposition. The result for the cokernels is analogous, by Remark 4.14 and Lemma 4.15 . 
Corollary 4.18. A direct consequence of Proposition 4.17 is the splitting of the index:

$$
\operatorname{Ind}\left(\mathcal{L}_{x \#_{T}^{0} y}\right)=\operatorname{Ind}\left(\mathcal{L}_{x}\right)+\operatorname{Ind}\left(\mathcal{L}_{y}\right)
$$

This can be rephrased as

$$
\operatorname{Ind}_{e}\left(\mathcal{L}_{x \#_{T}^{0} y}\right)=\operatorname{Ind}_{e}\left(\mathcal{L}_{x}\right)+\operatorname{Ind}_{e}\left(\mathcal{L}_{y}\right)-1,
$$

in the case of gluing across a free orbit $O_{b}$, or as

$$
\operatorname{Ind}_{e}\left(\mathcal{L}_{x \#_{T}^{0} y}\right)=\operatorname{Ind}_{e}\left(\mathcal{L}_{x}\right)+\operatorname{Ind}_{e}\left(\mathcal{L}_{y}\right)
$$

in the case of the fixed point $b=\theta$, with Ind Is $_{e}$ in Theorem 3.8, satisfying

$$
\operatorname{Ind}_{e}\left(\mathcal{L}_{\mathbb{A}, \Psi}\right)=\mu\left(O_{a}\right)-\mu\left(O_{b}\right)+1-\operatorname{dim}\left(G_{a}\right) .
$$

Proof. The index of the linearization $\mathcal{L}_{x \#_{T}^{0} y}$ can be computed as

$$
\operatorname{Ind}\left(\mathcal{L}_{x \#_{T}^{0} y}\right)=\operatorname{dim}\left(\operatorname{ApprKer}\left(\mathcal{L}_{x \#_{T}^{0} y}\right)\right)-\operatorname{dim}\left(\operatorname{ApprCoker}\left(\mathcal{L}_{x \#_{T}^{0} y}\right)\right) .
$$

This can be seen, for instance, by the supertrace formula for the index. Proposition 4.17 then gives the result. The statement for $I n d_{e}$ follows from the relation

$$
\operatorname{Ker}\left(\mathcal{L}_{x}\right) \cong \operatorname{Ker}_{e}\left(\mathcal{L}_{x}\right) / \mathbb{R} \text { and } \operatorname{Ker}\left(\mathcal{L}_{y}\right) \cong \operatorname{Ker}_{e}\left(\mathcal{L}_{y}\right) / \mathbb{R},
$$

with $\mathrm{Ker}_{e}$ the space of extended $L_{1, \delta}^{2}$ solutions as described in Theorem 3.8.

All the previous discussion makes no assumption on the cokernels of the linearizations $\mathcal{L}_{x}$ and $\mathcal{L}_{y}$, so it applies equally to the unobstructed case analyzed in the rest of this section (the case of the moduli spaces $\hat{\mathcal{M}}\left(O_{a}, O_{b}\right)$ of flow lines), and to the obstructed case of the moduli spaces $\mathcal{M}\left(O_{a}, O_{a^{\prime}}\right)$ and $\mathcal{M}^{P}\left(O_{a}, O_{b}\right)$ introduced in Section 6, Theorem 6.1. The obstructed case is discussed in Lemma 6.14 and Proposition 6.17.

For the remaining of this section we assume that $\operatorname{Coker}\left(\mathcal{L}_{x}\right)=0$ and $\operatorname{Coker}\left(\mathcal{L}_{y}\right)=0$. When these conditions are satisfied, we say that our gluing theory is unobstructed. In this case, we obtain the following result. 
Proposition 4.19. Let $K$ be a compact set

$$
K \subset \hat{\mathcal{M}}\left(O_{a}, O_{b}\right) \times_{O_{b}} \hat{\mathcal{M}}\left(O_{b}, O_{c}\right) .
$$

There exist a bound $T_{0}(K)>0$ such that, for all $T \geq T_{0}(K)$ and for all broken trajectories

$$
\left(\left(A_{1}(t), \psi_{1}(t)\right),\left(A_{2}(t), \psi_{2}(t)\right)\right) \in K,
$$

the Fredholm operator $\mathcal{L}_{x \#_{T}^{0} y}$

$$
\mathcal{L}_{x \#_{T}^{0} y}: \mathcal{T}_{1_{x \#_{T}^{0} y}} \rightarrow \mathcal{T}_{0 x \#_{T}^{0} y}
$$

is surjective. We are also assuming that $\operatorname{Coker}\left(\mathcal{L}_{x}\right)=0$ and $\operatorname{Coker}\left(\mathcal{L}_{y}\right)=0$. Then the composition of the pre-gluing map $\#_{T}^{0}$ with the orthogonal projection on the actual kernel $\operatorname{Ker}\left(\mathcal{L}_{x \#_{T}^{0} y}\right)$ gives an isomorphism

$$
\operatorname{Ker}\left(\mathcal{L}_{x}\right) \times \operatorname{Ker}\left(\mathcal{L}_{y}\right) \stackrel{\cong}{\rightarrow} \operatorname{Ker}\left(\mathcal{L}_{x \#_{T}^{0} y}\right) .
$$

Proof. We know that $\mathcal{L}_{x \#_{T}^{0} y}$ is Fredholm of index $\mu\left(O_{a}\right)-\mu\left(O_{c}\right)$. We also know that $\operatorname{dim} \operatorname{Ker}_{e}\left(\mathcal{L}_{x}\right)=\mu\left(O_{a}\right)-\mu\left(O_{b}\right)+\operatorname{dim}\left(O_{a}\right)$ and $\operatorname{dim} \operatorname{Ker}_{e}\left(\mathcal{L}_{y}\right)=$ $\mu\left(O_{b}\right)-\mu\left(O_{c}\right)+\operatorname{dim}\left(O_{b}\right)$.

We need to know that for any pair $x=\left(\mathbb{A}_{1}, \Psi_{1}\right)$ and $y=\left(\mathbb{A}_{2}, \Psi_{2}\right)$ there is a bound $T_{0}=T(x, y)$ such that $\mathcal{L}_{x \#_{T}^{0} y}$ is surjective for $T \geq T_{0}$. The compactness of $K$ will ensure that there is a uniform such bound $T(K)$.

Lemma 4.13 provides the crucial step in the argument: the linearization $\mathcal{L}_{x \#_{T}^{0} y}$ has a bounded right inverse when restricted to the orthogonal complement of $F_{\#}\left(\operatorname{Ker}\left(\mathcal{L}_{x}\right) \times \operatorname{Ker}\left(\mathcal{L}_{y}\right)\right)$.

Now Proposition 4.19 follows form the splitting of the index 4.18, since we obtain the estimate

$$
\begin{aligned}
\operatorname{dim} \operatorname{Ker}\left(\mathcal{L}_{x \#_{T}^{0} y}\right) & \leq \operatorname{dim} \operatorname{Ker}\left(\mathcal{L}_{x}\right)+\operatorname{dim} \operatorname{Ker}\left(\mathcal{L}_{y}\right) \\
& =\operatorname{Ind}\left(\mathcal{L}_{x}\right)+\operatorname{Ind}\left(\mathcal{L}_{y}\right)=\operatorname{Ind}\left(\mathcal{L}_{x \#_{T}^{0} y}\right) \\
& \leq \operatorname{dim} \operatorname{Ker}\left(\mathcal{L}_{x \#_{T}^{0} y}\right)
\end{aligned}
$$

hence

$$
\operatorname{dim} \operatorname{Ker}\left(\mathcal{L}_{x \#_{T}^{0} y}\right)=\operatorname{dim} \operatorname{Ker}\left(\mathcal{L}_{x}\right)+\operatorname{dim} \operatorname{Ker}\left(\mathcal{L}_{y}\right)
$$


In terms of the extended kernels, the isomorphism (51) gives rise to the isomorphism

$$
\operatorname{Ker}_{e}\left(\mathcal{L}_{x \#_{T}^{0} y}\right) \cong \operatorname{Ker}_{e}\left(\mathcal{L}_{x}\right) \times_{\mathbb{R}} \operatorname{Ker}_{e}\left(\mathcal{L}_{y}\right) .
$$

The space on the right hand side is the tangent space to the fibered product

$$
\mathcal{M}\left(O_{a}, O_{b}\right) \times_{O_{b}} \mathcal{M}\left(O_{b}, O_{c}\right) .
$$

Now we can proceed to give a proof of Theorem 4.9.

Proof of Theorem 4.9. Given the approximate solution obtained via the pregluing map $\#_{T}^{0}$, we prove that this can be perturbed to an actual solution. Similar arguments are presented in [18], [57].

In order to deform a pre-glued approximate solution to an actual solution we need to construct a projection of pre-glued solutions onto $\mathcal{M}\left(O_{a}, O_{c}\right)$. Thus we need a normal bundle to $\mathcal{M}\left(O_{a}, O_{c}\right)$ inside $\mathcal{B}_{k \delta}^{0}\left(O_{a}, O_{c}\right)$. We proceed according to the following construction. Let $\mathcal{U}\left(O_{a}, O_{c}\right)$ be the image in $\mathcal{B}^{0}\left(O_{a}, O_{c}\right)$ of the pre-gluing map $\#_{T}^{0}$ defined on $\mathcal{M}\left(O_{a}, O_{b}\right) \times O_{b} \mathcal{M}\left(O_{b}, O_{c}\right)$. Consider the Hilbert bundles $\mathcal{T}_{1}$ and $\mathcal{T}_{0}$ given by the $L_{1, \delta}^{2}$ and $L_{0, \delta}^{2}$ tangent bundles of $\mathcal{A}\left(O_{a}, O_{c}\right)$. Choose a slice

$$
\mathcal{S}_{\Gamma_{a c}} \subset \mathcal{A}\left(O_{a}, O_{c}\right),
$$

such that the pre-glued element $x \#_{T}^{0} y$ lies in a ball of radius $r$ in $\mathcal{A}\left(O_{a}, O_{c}\right)$ centered at $\Gamma_{a c}$. The flow equation

$$
f\left(x \#_{T}^{0} y\right)=\left\{\begin{array}{l}
D_{\mathbb{A}_{1} \#_{T}^{0} \mathbb{A}_{2}}\left(\Psi_{1} \#_{T}^{0} \Psi_{2}\right) \\
F_{\mathbb{A}_{1} \#_{T}^{0} \mathbb{A}_{2}}^{+}-\left(\Psi_{1} \#_{T}^{0} \Psi_{2}\right) \cdot \overline{\left(\Psi_{1} \#_{T}^{0} \Psi_{2}\right)}-i \mu-P_{\left(\mathbb{A}_{1} \#_{T}^{0} \mathbb{A}_{2}, \Psi_{1} \#_{T}^{0} \Psi_{2}\right)} \\
G_{\Gamma_{a c}}^{*}\left(\mathbb{A}_{1} \#_{T}^{0} \mathbb{A}_{2}, \Psi_{1} \#_{T}^{0} \Psi_{2}\right)
\end{array}\right.
$$

induces a bundle map $f: \mathcal{T}_{1} \rightarrow \mathcal{T}_{0}$. The linearization $\mathcal{L}_{x \#_{T}^{0} y}$ is the fiber derivative of $f$.

We assume here that the linearizations $\mathcal{L}_{x}$ and $\mathcal{L}_{y}$ are surjective. Thus, consider the space

$$
\mathcal{K}=\bigcup_{K \times\left[T_{0}, \infty\right)} \operatorname{Ker}_{e}\left(\mathcal{L}_{x}\right) \times_{\mathbb{R}} \operatorname{Ker}_{e}\left(\mathcal{L}_{y}\right) .
$$

The image of $\mathcal{K}$ under the linearization $F_{\#}$ of the pre-gluing map defines a sub-bundle of $\mathcal{T}_{1}$. We consider the space $\mathcal{T}_{x \#_{T}^{0} y}^{\perp}$ of elements of the tangent space $\mathcal{T}_{1}$ at the point $x \#_{T}^{0} y$ that are orthogonal to

$$
\mathcal{T}_{x \#_{T}^{0} y}=F_{\#}\left(\operatorname{Ker}_{e}\left(\mathcal{L}_{x}\right) \times_{\mathbb{R}} \operatorname{Ker}_{e}\left(\mathcal{L}_{y}\right)\right) .
$$


The space $\mathcal{T}^{\perp}$ gives the normal bundle of the gluing construction. In the case of the fixed point $b=\theta$, we have

$$
\mathcal{K}=\cup_{K \times\left[T_{0}, \infty\right)} \operatorname{Ker}_{e}\left(\mathcal{L}_{x}\right) \times \operatorname{Ker}\left(\mathcal{L}_{y}\right)
$$

and the normal bundle of the gluing construction given by

$$
\mathcal{T}_{x \#_{T}^{0} y}^{\perp}=\left(F_{\# T}\left(\operatorname{Ker}_{e}\left(\mathcal{L}_{x}\right) \times \operatorname{Ker}\left(\mathcal{L}_{y}\right)\right)\right)^{\perp} .
$$

Now we want to define the actual gluing map \# that provides a solution of the flow equations in $\mathcal{M}\left(O_{a}, O_{c}\right)$. This means that we want to obtain a section $\sigma$ of $\mathcal{T}_{1}$ such that the image under the bundle homomorphism given by the flow equation is zero in $\mathcal{T}_{0}$. Moreover, we want this element

$$
\sigma(x, y, T)=\sigma\left(\mathbb{A}_{1}, \Psi_{1}, \mathbb{A}_{2}, \Psi_{2}, T\right)
$$

to converge to zero sufficiently rapidly as $T \rightarrow \infty$, so that the glued solution will converge to the broken trajectory in the limit $T \rightarrow \infty$.

The perturbation of the approximate solution to an actual solution can be obtained as a fixed point theorem in Banach spaces, via the following contraction principle.

Remark 4.20. Suppose given a smooth map $f: E \rightarrow F$ between Banach spaces of the form

$$
f(x)=f(0)+D f(0) x+N(x)
$$

with $\operatorname{Ker}(D f(0))$ finite dimensional, with a right inverse $D f(0) \circ G=I d_{F}$, and with the nonlinear part $N(x)$ satisfying the estimate

$$
\|G N(x)-G N(y)\| \leq C(\|x\|+\|y\|)\|x-y\|
$$

for some constant $C>0$ and $x$ and $y$ in a small neighborhood $B_{\epsilon(C)}(0)$. Then, with the initial condition $\|G(f(0))\| \leq \epsilon / 2$, there is a unique zero $x_{0}$ of the map $f$ in $B_{\epsilon}(0) \cap G(F)$. This satisfies $\left\|x_{0}\right\| \leq \epsilon$.

The map $f$ is given in our case by the flow equation, viewed as a bundle homomorphism $\mathcal{T}_{1} \mapsto \mathcal{T}_{0}$.

Proposition 4.21. Lemma 4.13 and Proposition 4.19 imply that the linearization $\mathcal{L}_{x \#_{T}^{0} y}$ is uniformly invertible on the orthogonal complement of the actual kernel

$$
\mathcal{T}_{x \#_{T}^{0} y}^{\perp}=\operatorname{Ker}_{e}\left(\mathcal{L}_{x \#_{T}^{0} y}\right)^{\perp} \cong\left(F_{\# T}\left(\operatorname{Ker}_{e}\left(\mathcal{L}_{x}\right) \times_{\mathbb{R}} \operatorname{Ker}_{e}\left(\mathcal{L}_{y}\right)\right)\right)^{\perp} .
$$


Consider the right inverse map of $\mathcal{L}=\mathcal{L}_{x \#_{T}^{0} y}$ restricted to $\mathcal{T}^{\perp}$,

$$
G: \mathcal{T}_{0} \rightarrow \mathcal{T}^{\perp}
$$

There is a $T(K)$ and a constant $C>0$ independent of $T$, such that we have

$$
\left\|G_{\chi} \xi\right\|_{L_{1, \delta}^{2}} \leq C\|\xi\|_{L_{0, \delta}^{2}}
$$

for $\chi=(x, y, T) \in K \times[T(K), \infty)$. Moreover, we can write $f$ as a sum of a linear and a non-linear term, where the linear term is $\mathcal{L}$ and the nonlinear term is

$$
N_{(A(t), \psi(t))}(\alpha, \phi)=\left(\sigma(\phi, \phi)+\mathcal{N} q_{(A(t), \psi(t))}(\alpha, \phi), \alpha \cdot \phi\right) .
$$

Here we write the perturbation $q$ of equation (19) as sum of a linear and a non-linear term, $2 q=\mathcal{D} q+\mathcal{N} q$. Then the conditions of Remark 4.20 are satisfied. This provides the existence of a unique correction term

$$
\sigma\left(\mathbb{A}_{1}, \Psi_{1}, \mathbb{A}_{2}, \Psi_{2}, T\right) \in B_{\epsilon}(0) \cap \mathcal{T}^{\perp}
$$

satisfying $f(\sigma)=0$. This element $\sigma$ is smooth and it decays to zero when $T$ is very large, as proved in Section 6 , using the estimate (103). This means that the glued solution converges to the broken trajectory $(x, y)$ for $T \rightarrow \infty$. The gluing map is given by

$$
\left(A_{1} \#_{T} A_{2}, \psi_{1} \#_{T} \psi_{2}\right)=\left(A_{1} \#_{T}^{0} A_{2}, \psi_{1} \#_{T}^{0} \psi_{2}\right)+\sigma\left(\mathbb{A}_{1}, \Psi_{1}, \mathbb{A}_{2}, \Psi_{2}, T\right),
$$

with a rate of decay

$$
\left\|\sigma\left(\mathbb{A}_{1}, \Psi_{1}, \mathbb{A}_{2}, \Psi_{2}, T\right)\right\|_{L_{1, \delta}^{2}} \leq C e^{-\delta T}
$$

as $T \rightarrow \infty$.

In Section 6, in Lemma 6.14 and Proposition 6.17, we shall consider a similar fixed point problem in the presence of obstructions coming form non-vanishing cokernels. The proof of Proposition 4.21 is given in Section 6, after Lemma 6.14, since it follows from the more general case discussed there. The rate of decay $\left\|\sigma\left(\mathbb{A}_{1}, \Psi_{1}, \mathbb{A}_{2}, \Psi_{2}, T\right)\right\|_{1, \delta} \leq C e^{-\delta T}$ as $T \rightarrow \infty$ is derived in Lemma 6.14 in Section 6.2.

Remark 4.22. Notice that, if we have a nontrivial cokernel of $\mathcal{L}_{x}$ or $\mathcal{L}_{y}$, then not only $\mathcal{T}^{\perp}$ is not be a bundle, but in general $\mathcal{L}_{x \#_{T}^{0} y}$ may not be invertible on all of the orthogonal complement of the actual kernel. We still 
know that $\mathcal{L}_{x \#_{T}^{0} y}$ is uniformly invertible on the orthogonal complement of the approximate kernel, but in this case the kernel is only a proper subspace of the approximate kernel. Thus there may be elements $\chi=(x, y, T)$ in $K \times\left[T_{0}, \infty\right)$ that cannot be perturbed to an actual solution. In fact, as seen in the proof of Lemma 4.13 , the condition that the composition $\tilde{F}_{\#_{T}}$ of the linearization $F_{\#_{T}}$ of the pre-gluing map $\#_{T}^{0}$ with the orthogonal projection onto $\operatorname{Ker}\left(\mathcal{L}_{x \#_{T}^{0} y}\right)$ gives an isomorphism

$$
\operatorname{Ker}_{e}\left(\mathcal{L}_{x}\right) \times_{\mathbb{R}} \operatorname{Ker}_{e}\left(\mathcal{L}_{y}\right) \stackrel{\cong}{\rightarrow} \operatorname{Ker}_{e}\left(\mathcal{L}_{x \#_{T}^{0} y}\right)
$$

is equivalent to the condition that

$$
\operatorname{Coker}\left(\mathcal{L}_{x}\right) \times \operatorname{Coker}\left(\mathcal{L}_{y}\right) \cong \operatorname{Coker}\left(\mathcal{L}_{x \#_{T}^{0} y}\right) .
$$

However, in the general case, where we may have non-trivial Coker $\left(\mathcal{L}_{x}\right)$ and Coker $\left(\mathcal{L}_{y}\right)$ we expect to have the isomorphism only at the level of the approximate kernel and cokernel, whereas the actual kernel and cokernel satisfy the weaker condition

$$
\operatorname{dim} \operatorname{Ker}\left(\mathcal{L}_{x \#_{T}^{0} y}\right)=\operatorname{dim} \operatorname{Ker}\left(\mathcal{L}_{x}\right)+\operatorname{dim} \operatorname{Ker}\left(\mathcal{L}_{y}\right)-k,
$$

for some $k \geq 0$, and

$$
\operatorname{dim} \operatorname{Coker}\left(\mathcal{L}_{x \#_{T}^{0} y}\right)=\operatorname{dim} \operatorname{Coker}\left(\mathcal{L}_{x}\right)+\operatorname{dim} \operatorname{Coker}\left(\mathcal{L}_{y}\right)-k,
$$

according to the computation of Lemma 4.13. This case occurs for instance in the case discussed in Section 6, Theorem 6.21 and Theorem 6.22.

To continue with the proof of Theorem 4.9, we now look at the induced map

$$
\hat{\#}: \hat{K} \times\left[T_{0}, \infty\right) \rightarrow \hat{\mathcal{M}}\left(O_{a}, O_{c}\right) .
$$

Recall that we have an identification of the moduli spaces $\hat{\mathcal{M}}\left(O_{a}, O_{b}\right)$ with the balanced moduli spaces $\mathcal{M}^{\text {bal }}\left(O_{a}, O_{b}\right)$ of classes in $\mathcal{M}\left(O_{a}, O_{b}\right)$ that satisfy the equal energy condition (29). Thus, we can define the induced gluing map \# by restricting the gluing map \# of (55) to the subspace

$$
\mathcal{M}^{b a l}\left(O_{a}, O_{b}\right) \times O_{b} \mathcal{M}^{b a l}\left(O_{b}, O_{c}\right) \subset \mathcal{M}\left(O_{a}, O_{b}\right) \times O_{b} \mathcal{M}\left(O_{b}, O_{c}\right),
$$

and then composing the image $x \#_{T} y \in \mathcal{M}\left(O_{a}, O_{c}\right)$ with a time translation that determines an element $\widehat{x \#_{T} y} \in \mathcal{M}^{b a l}\left(O_{a}, O_{c}\right)$. We define $x \hat{\#}_{T} y=$

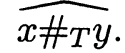


The identification $\hat{\mathcal{M}}\left(O_{a}, O_{b}\right) \cong \mathcal{M}^{b a l}\left(O_{a}, O_{b}\right)$ determines an identification of the tangent spaces

$$
\mathcal{T}_{x} \mathcal{M}\left(O_{a}, O_{b}\right) \cong \mathcal{T}_{x} \hat{\mathcal{M}}\left(O_{a}, O_{b}\right) \oplus \mathbb{R} \cdot U_{x}
$$

for $x \in \mathcal{M}^{\text {bal }}\left(O_{a}, O_{b}\right)$. Here we have

$$
U_{x}=\Pi_{\mathcal{S}} \nabla \mathcal{C}_{\rho}\left(\lambda_{1}^{+} x(t)\right),
$$

where the gauge transformation $\lambda_{1}^{+}$is as in (34) and $\Pi_{\mathcal{S}}$ is the projection on the tangent space to the slice $\mathcal{T}\left(\mathcal{S}_{\Gamma_{a b}}\right)$. Here $\mathcal{T}$ denotes the $L_{1, \delta}^{2}$ tangent space.

In order to prove that $\hat{\#}$ is a local diffeomorphism, we first prove that the linearization $\tilde{F}_{\hat{\#}_{T}}$ is an isomorphism. Then we prove that the linearization of the gluing map is sufficiently close to $\tilde{F}_{\hat{\#}_{T}}$, by estimating the variation of the error term with respect to parameters (the solutions in the component spaces $\mathcal{M}\left(O_{a}, O_{b}\right), \mathcal{M}\left(O_{a}, O_{c}\right)$, and $\left.T\right)$. Finally, we prove injectivity of $\#_{T}$.

Recall that we have tangent spaces

$$
\mathcal{T}_{x} \mathcal{M}\left(O_{a}, O_{b}\right) \cong \operatorname{Ker}_{e}\left(\mathcal{L}_{x}\right) \text { and } \mathcal{T}_{y} \mathcal{M}\left(O_{a}, O_{b}\right) \cong \operatorname{Ker}_{e}\left(\mathcal{L}_{y}\right) .
$$

We have a decomposition as in (57) with an element $U_{y}$ defined analogously. Moreover, we have the isomorphism of Proposition 4.19, cf. Remark 4.22, which gives an isomorphism

$$
\tilde{F}_{\#_{T}}: \mathcal{T}_{(x, y)}\left(\mathcal{M}\left(O_{a}, O_{b}\right) \times_{O_{b}} \mathcal{M}\left(O_{b}, O_{c}\right)\right) \stackrel{\cong}{\rightarrow} \mathcal{T}_{x \#_{T} y} \mathcal{M}\left(O_{a}, O_{c}\right),
$$

where $\tilde{F}_{\#_{T}}$ is the composition of the linearization $F_{\# T}$ of the pre-gluing map with the projection onto the kernel $\operatorname{Ker}_{e}\left(\mathcal{L}_{x \#_{T} y}\right)$.

We can consider the subspace

$$
\mathcal{T}_{(x, y)}\left(\hat{\mathcal{M}}\left(O_{a}, O_{b}\right) \times_{O_{b}} \hat{\mathcal{M}}\left(O_{b}, O_{c}\right)\right) \oplus \mathbb{R} \cdot\left(U_{x},-U_{y}\right)
$$

inside the space

$$
\begin{aligned}
\mathcal{T}_{(x, y)}\left(\mathcal{M}\left(O_{a}, O_{b}\right) \times_{O_{b}} \mathcal{M}\left(O_{b}, O_{c}\right)\right) \cong & \mathcal{T}_{(x, y)}\left(\hat{\mathcal{M}}\left(O_{a}, O_{b}\right) \times_{O_{b}} \hat{\mathcal{M}}\left(O_{b}, O_{c}\right)\right) \\
& \mathbb{R} \cdot U_{x} \oplus \mathbb{R} \cdot U_{y} .
\end{aligned}
$$

Here we have $x$ and $y$ in $\mathcal{M}^{\text {bal }}\left(O_{a}, O_{b}\right)$ and $\left.\mathcal{M}^{\text {bal }}\left(O_{b}, O_{c}\right)\right)$, respectively.

Claim. For all $T \geq T_{0}$, sufficiently large, the restriction of the isomorphism (58) to the subspace (59) gives the desired isomorphism

$$
\tilde{F}_{\hat{\#}_{T}}: \mathcal{T}_{(x, y)}\left(\hat{\mathcal{M}}\left(O_{a}, O_{b}\right) \times{ }_{O_{b}} \hat{\mathcal{M}}\left(O_{b}, O_{c}\right)\right) \oplus \mathbb{R} \cdot\left(U_{x},-U_{y}\right) \cong \mathcal{T}_{x \hat{\#}_{T} y} \hat{\mathcal{M}}\left(O_{a}, O_{c}\right) .
$$


Proof of Claim. Suppose there exist a sequence $T_{n} \rightarrow \infty$ such that there exist $\left(\xi_{n}, \eta_{n}, \tau_{n}\right)$ in the space (59), with the property that

$$
F_{\#_{T_{n}}}\left(\xi_{n}, \eta_{n}, \tau_{n}\right) \in \mathbb{R} \cdot U_{T_{n}}
$$

where $U_{T_{n}}$ is defined as

$$
U_{T_{n}}=\Pi_{\mathcal{S}} \nabla \mathcal{C}_{\rho}\left(\lambda_{n}\left(x \hat{\#}_{T_{n}} y\right)(t)\right),
$$

with gauge transformations $\lambda_{n}$ as in (34) and the projection $\Pi_{\mathcal{S}}$ on the slice $\mathcal{S}_{\Gamma_{a c}}$. We are using the identification

$$
\mathcal{T}_{x \#_{T_{n}} y} \mathcal{M}\left(O_{a}, O_{c}\right) \cong \mathcal{T}_{x \#_{T_{n}} y} \hat{\mathcal{M}}\left(O_{a}, O_{c}\right) \oplus \mathbb{R} \cdot U_{T_{n}} .
$$

We have

$$
F_{\# T_{n}}\left(\xi_{n}, \eta_{n}, \tau_{n}\right)=\tilde{\tau}_{n} U_{T_{n}},
$$

for some $\tilde{\tau}_{n} \in \mathbb{R}$. We can normalize the elements $\left(\xi_{n}, \eta_{n}, \tau_{n}\right)$ so that $\tilde{\tau}_{n}=1$ for all $n$. By the result of Proposition 4.21, this implies that the pre-glued elements satisfy

$$
\left\|F_{\#_{T_{n}}^{0}}\left(\xi_{n}, \eta_{n}\right)+\tau_{n} F_{\#_{T_{n}}^{0}}\left(U_{x},-U_{y}\right)-F_{\#_{T_{n}}^{0}}\left(U_{x}, U_{y}\right)\right\|_{L_{1, \delta\left(T_{n}\right)}^{2}} \rightarrow 0
$$

as $n \rightarrow \infty$. We are using the fact that the error term in the gluing map (55) decays to zero as $T_{n} \rightarrow \infty$ as in Proposition 4.21 and in (103). This then implies the following convergence

$$
\left\|\xi_{n}+\left(\tau_{n}-1\right) U_{x}\right\|_{L_{1, \delta}^{2}\left(Y \times\left(-\infty, T_{n}-1\right]\right)} \rightarrow 0
$$

and

$$
\left\|\eta_{n}-\left(1+\tau_{n}\right) U_{y}\right\|_{L_{1, \delta}^{2}\left(Y \times\left[+1-T_{n}, \infty\right)\right)} \rightarrow 0,
$$

where we use the norm estimate for the map (46).

This gives a contradiction, according to the decomposition (57), which prescribes that $\xi_{n} \perp U_{x}$ and $\eta_{n} \perp U_{y}$, cf. Proposition 2.56 of [57].

We can estimate explicitly the norm of the isomorphism $\tilde{F}_{\hat{\#}_{T}}$. This is bounded by the product of the norm of the linearization $F_{\#_{T}}$ at the pre-glued solution (44), the norm of the isomorphism $\operatorname{Ker}\left(\mathcal{L}_{x \#_{T}^{0} y}\right) \cong \operatorname{Ker}\left(\mathcal{L}_{x \#_{T} y}\right)$, and the norm of the isomorphism $\operatorname{Ker}\left(\mathcal{L}_{x \#_{T} y}\right) \cong \operatorname{Ker}\left(\mathcal{L}_{x \hat{\#}_{T} y}\right)$ given by time translation. The first norm is bounded uniformly in $T$, if we use the rescaled norms $L_{1, \delta\left(T_{n}\right)}^{2}$ on the tangent space of $\mathcal{M}\left(O_{a}, O_{c}\right)$. The second norm is bounded by a constant $C_{2}=C_{2}(K)$ over the compact set $K$ because of the decay of the error term in the gluing map (55), cf. (103). The third 
norm is bounded by $C e^{\delta \tau\left(x \#_{T} y\right)}$, where $\tau\left(x \#_{T} y\right)$ is the unique time shift that maps $x \#_{T} y$ to the element $\widehat{x \#_{T} y}$ satisfying the equal energy condition (29). Thus, on the compact set $K$ this norm is also bounded by a term $C e^{\delta \cdot \tau(K)}$. Summarizing, we have obtained the estimate $\left\|\tilde{F}_{\hat{\#}_{T}}\right\| \leq C$, on the operator norm of $\tilde{F}_{\hat{\#}_{T}}$, uniformly in $T$, for

$$
\tilde{F}_{\hat{\#}_{T}}: \mathcal{T}_{1, \delta}\left(\mathcal{M}\left(O_{a}, O_{b}\right)\right) \times \mathcal{T}_{1, \delta}\left(\mathcal{M}\left(O_{b}, O_{c}\right)\right) \rightarrow \mathcal{T}_{1, \delta(T)} \mathcal{M}\left(O_{a}, O_{c}\right),
$$

or $\left\|\tilde{F}_{\hat{\#}_{T}}\right\| \leq C e^{-\delta T}$, for

$$
\tilde{F}_{\hat{\#} \boldsymbol{T}}: \mathcal{T}_{1, \delta}\left(\mathcal{M}\left(O_{a}, O_{b}\right)\right) \times \mathcal{T}_{1, \delta}\left(\mathcal{M}\left(O_{b}, O_{c}\right)\right) \rightarrow \mathcal{T}_{1, \delta} \mathcal{M}\left(O_{a}, O_{c}\right) .
$$

The linearization $\tilde{F}_{\hat{\#}_{T}}$ is an approximation to the linearization of the gluing map. In order to show that the latter is an isomorphism, we consider derivatives of the correction term, and show that they are small compared to $F_{\#_{T}}$. Similar arguments are given in [18] §7.2.4-7.2.6.

We have the correction term $\sigma(x, y, T)=(\alpha, \Phi)$, as in (55), such that $\left[x \#_{T}^{0} y+(\alpha, \Phi)\right] \in \mathcal{M}\left(O_{a}, O_{c}\right)$. This term satisfies (99), which we write here simply as

$$
f+\mathcal{L} \sigma+\mathcal{N} \sigma=0
$$

We write $\sigma=\mathcal{L}^{*} \tilde{\sigma}$, with $\tilde{\sigma}=(\beta, \xi)$, as in Lemma 6.14 . If we denote by $D$ differentiation with respect to parameters, we obtain from (60)

$$
\left(\mathcal{L} \mathcal{L}^{*}+\mathcal{N} \mathcal{L}^{*}\right) D \tilde{\sigma}=-\left(D f+D\left(\mathcal{L} \mathcal{L}^{*}+\mathcal{N} \mathcal{L}^{*}\right) \tilde{\sigma}\right) .
$$

We denote the right hand side with $\mathcal{Q}(D f, \tilde{\sigma})$. In the contraction neighborhood of Lemma 6.14 and Proposition 4.21, we can estimate, for some $C>0$

$$
\|\mathcal{Q}(D f, \tilde{\sigma})\|_{L_{1, \delta}^{2}} \leq C\left\|F_{\#_{T}}\right\| e^{-\delta T} .
$$

Here $\left\|F_{\# T}\right\|$ is the operator norm of $F_{\#_{T}}$ acting between $L_{1, \delta}^{1}$ spaces. The estimate (61) is obtained by combining the estimate $\|\tilde{\sigma}\| \leq C e^{-\delta T}$ for the correction term, as in Proposition 4.21, and the estimate $\left\|D\left(\mathcal{L L}^{*}+\mathcal{N} \mathcal{L}^{*}\right)\right\| \leq$ $C\left\|F_{\#_{T}}\right\|$, together with an estimate for the derivative $D f$ of the error term $f=f\left(x \#_{T}^{0} y\right)$ :

$$
\begin{aligned}
& \left\|\left.D f\right|_{(x, y)}(u, v)\right\|_{1, \delta} \\
& \leq\left\|\left.F_{\#_{T}}\right|_{(x, y)}\right\|\left(\left\|\mathcal{L}_{x \#_{T}^{0} y}\left(\rho^{-} u^{T}\right)\right\|_{1, \delta}+\left\|\mathcal{L}_{x \#_{T}^{0} y}\left(\rho^{+} v^{-T}\right)\right\|_{1, \delta}\right),
\end{aligned}
$$


which gives, by estimates like (45) and (47), $\|D f\|_{L_{1, \delta}^{2}} \leq C\left\|F_{\hat{\#}_{T}}\right\| e^{-\delta T}$.

Thus, from the identity

$$
D \tilde{\sigma}=-\left(\mathcal{L} \mathcal{L}^{*}\right)^{-1}(1-\Pi)\left(\mathcal{N} \mathcal{L}^{*} D \tilde{\sigma}+\mathcal{Q}(D f, \tilde{\sigma})\right),
$$

with $\Pi=\Pi\left(\mu, x \#_{T}^{0} y\right)$ as in Lemma 6.14 , we obtain, for some $C>0$,

$$
\|D \tilde{\sigma}\|_{L_{1, \delta}^{2}} \leq C\left\|F_{\#_{T}}\right\| e^{-\delta T}
$$

We still have to prove the injectivity of the map $\#_{T}$ for all sufficiently large $T \geq T_{0}$. Suppose there exist a sequence $T_{k} \rightarrow \infty$ and elements $\left(x_{1, k}, y_{1, k}\right) \neq\left(x_{2, k}, y_{2, k}\right)$ in $\mathcal{M}^{b a l}\left(O_{a}, O_{b}\right) \times_{O_{b}} \mathcal{M}^{b a l}\left(O_{b}, O_{c}\right)$, such that

$$
x_{1, k} \hat{\#}_{T_{k}} y_{1, k}=x_{2, k} \hat{\#}_{T_{k}} y_{2, k}
$$

for all $k$. Then, there exist a based $L_{2, \delta}^{2}$ gauge transformation $\lambda$ on $Y \times \mathbb{R}$ such that we have

$$
\lim _{k \rightarrow \infty}\left\|x_{1, k} \#_{T_{k}}^{0} y_{1, k}-\lambda\left(x_{2, k} \#_{T_{k}}^{0} y_{2, k}\right)\right\|_{L_{1, \delta}^{2}}=0 .
$$

We are using here the fact that the time shifts agree as $T_{k} \rightarrow \infty$. Upon passing to a subsequence, we know that there are limits $x_{i}=\lim _{k} x_{i, k}$ and $y_{i}=\lim _{k} y_{i, k}$, for $i=1,2$, by the assumed compactness of $\hat{K}$. We obtain

$$
x_{1} \hat{\#}_{T_{k}} y_{1}=x_{1} \hat{\#}_{T_{k}} y_{2},
$$

for all $T_{k}$, but for $T_{k} \rightarrow \infty$ we have convergence of $x_{i} \hat{\#}_{T_{k}} y_{i}$ to the broken trajectory $\left(x_{i}, y_{i}\right)$. We obtain $\left(x_{1}, y_{1}\right)=\left(x_{2}, y_{2}\right)=(x, y)$. Now consider the elements $\left(u_{i, k}, v_{i, k}\right)=\left(x_{i, k}-x, y_{i, k}-y\right)$, for $i=1,2$. They satisfy $\left(u_{1, k}, v_{1, k}\right) \neq\left(u_{2, k}, v_{2, k}\right)$ and

$$
\left.F_{\# T_{k}}\right|_{(x, y)}\left(u_{1, k}, v_{1, k}\right)=\left.F_{\#_{T_{k}}}\right|_{(x, y)}\left(u_{2, k}, v_{2, k}\right),
$$

for all $k$, which contradicts the fact that the map $F_{\#}$ is a local diffeomorphism, as we just proved. Notice that it is also possible to give an alternate proof of this fact by direct estimates, along the lines of [18], replacing our proof by contradiction: some may find that more appealing.

Now a comment about orientations. Notice that the isomorphism of Proposition 4.19 induces an isomorphism

$$
\Lambda^{\max } \operatorname{Ker}_{e}\left(\mathcal{L}_{x}\right) \otimes \Lambda^{\max } \operatorname{Ker}_{e}\left(\mathcal{L}_{y}\right) \rightarrow \Lambda^{\max } \operatorname{Ker}_{e}\left(\mathcal{L}_{x \#} y\right)
$$


Thus, in the unobstructed case we are considering, where all the cokernels are trivial, we obtain that the gluing map $\#_{T}$ is compatible with the orientations. Using the decompositions (57) we obtain that the gluing map $\hat{\#}_{T}$ is also compatible with the induced orientations on the moduli spaces $\hat{\mathcal{M}}\left(O_{a}, O_{b}\right)$, cf. Proposition 3.6 of [57]. Notice that the orientation on

$$
\left(\hat{\mathcal{M}}\left(O_{a}, O_{b}\right) \times O_{b} \hat{\mathcal{M}}\left(O_{b}, O_{c}\right)\right) \times \mathbb{R}
$$

induced, under the decomposition (57), by the product orientation on

$$
\mathcal{M}\left(O_{a}, O_{b}\right) \times_{O_{b}} \mathcal{M}\left(O_{b}, O_{c}\right)
$$

agrees with the pullback of the product orientation of

$$
\hat{\mathcal{M}}\left(O_{a}, O_{b}\right) \times \mathbb{R} \times \hat{\mathcal{M}}\left(O_{b}, O_{c}\right)
$$

under the map $(x, y, T) \rightarrow(x, T, y)$.

We now come to the last statement in Theorem 4.9. We prove that any sequence of trajectories in $\mathcal{M}\left(O_{a}, O_{c}\right)$ converging to a broken trajectory in $\mathcal{M}\left(O_{a}, O_{b}\right) \times O_{b} \mathcal{M}\left(O_{b}, O_{c}\right)$ lies eventually in the image of the gluing map.

This requires a preliminary statement about the endpoint maps

$$
e_{b}^{-}: \mathcal{M}\left(O_{a}, O_{b}\right) \rightarrow O_{b} \text { and } e_{b}^{+}: \mathcal{M}\left(O_{b}, O_{c}\right) \rightarrow O_{b} .
$$

Claim. Suppose given a sequence $\left(x_{n}, y_{n}\right)$ in

$$
K_{1} \times K_{2} \subset \mathcal{M}\left(O_{a}, O_{b}\right) \times \mathcal{M}\left(O_{b}, O_{c}\right),
$$

with $K_{1}$ and $K_{2}$ compact sets in the $L_{2, \delta^{-}}^{2}$ topology. Suppose that the endpoints $e_{b}^{-}\left(x_{n}\right)$ and $e_{b}^{+}\left(y_{n}\right)$ converge to the same element $x_{b}$ on the critical orbit $O_{b}$, in the $L_{1}^{2}$-topology on $\mathcal{B}^{0}$. Then there is a subsequence $\left(x_{n}^{\prime}, y_{n}^{\prime}\right)$ converging to an element $\left(x^{\prime}, y^{\prime}\right)$ in the fibered product $\mathcal{M}\left(O_{a}, O_{b}\right) \times O_{b} \mathcal{M}\left(O_{b}, O_{c}\right)$.

Proof of Claim. The compactness of $K_{1} \times K_{2}$ ensures the existence of a convergent subsequence $\left(x_{n}^{\prime}, y_{n}^{\prime}\right)$ with limit $\left(x^{\prime}, y^{\prime}\right)$. Moreover, we can estimate the distance

$$
\begin{aligned}
\left\|e_{b}^{-}\left(x^{\prime}\right)-e_{b}^{+}\left(y^{\prime}\right)\right\|_{L_{1}^{2}} \leq & \left\|e_{b}^{-}\left(x_{n}\right)-e_{b}^{-}\left(x^{\prime}\right)\right\|_{L_{1}^{2}} \\
& +\left\|e_{b}^{+}\left(y_{n}\right)-e_{b}^{+}\left(y^{\prime}\right)\right\|_{L_{1}^{2}}+\left\|e_{b}^{-}\left(x_{n}\right)-e_{b}^{+}\left(y_{n}\right)\right\|_{L_{1}^{2}} .
\end{aligned}
$$


The last term goes to zero by hypothesis, and the first and second term on the right hand side go to zero by the continuity of the endpoint maps. Thus, we have $e_{b}^{-}\left(x^{\prime}\right)=e_{b}^{+}\left(y^{\prime}\right)=x_{b}$.

Now we return to the statement on the range of the gluing map. This is the analogue in our setting of the method of continuity used in Section 7.3 of [18], and of the arguments of Lemma 4.5.1 and Section 4.6 of [46].

Suppose given a sequence $X_{k}$ of elements in $\mathcal{M}\left(O_{a}, O_{c}\right)$ which converges smoothly on compact sets (as in Theorem 4.1) to a broken trajectory $(x, y)$ in the fibered product $\mathcal{M}\left(O_{a}, O_{b}\right) \times O_{b} \mathcal{M}\left(O_{b}, O_{c}\right)$.

For a sequence of gluing parameters $T_{k} \rightarrow \infty$, let $\left(x_{k}, y_{k}\right)$ be the projection onto the slices $\mathcal{S}_{\Gamma_{a b}} \times \mathcal{S}_{\Gamma_{b c}}$,

$$
\mathcal{S}_{\Gamma_{a b}} \subset \mathcal{A}_{k, \delta}\left(O_{a}, O_{b}\right) \text { and } \mathcal{S}_{\Gamma_{b c}} \subset \mathcal{A}_{k, \delta}\left(O_{b}, O_{c}\right),
$$

of the cut off elements $\left(\rho_{1-T_{k}}^{-} X_{k}^{-T_{k}}, \rho_{-1+T_{k}}^{+} X_{k}^{T_{k}}\right)$. The elements $x_{k}$ and $y_{k}$ are no longer solutions of the Seiberg-Witten equations. However, we can choose the sequence $T_{k} \rightarrow \infty$ such that the elements $x_{k}$ and $y_{k}$ lie respectively within neighborhoods of radius $\epsilon / 4$ of the solutions $x$ and $y$, in the $L_{2, \delta^{-}}^{2}$ norm. We choose $\epsilon$ so that it satisfies

$$
\epsilon \leq \min \left\{\epsilon_{a c}, \epsilon_{a b}, \epsilon_{b c}\right\} .
$$

Here we have $\epsilon_{a b}$ and $\epsilon_{b c}$ determined as in Lemma 6.14, for the contraction principle for the fixed point problem (102) in $\mathcal{B}_{2, \delta}\left(O_{a}, O_{b}\right)$ and in $\mathcal{B}_{2, \delta}\left(O_{b}, O_{c}\right)$, respectively. Similarly, the constant $\epsilon_{a c}$ is the constant for the contraction principle of Lemma 6.14 in $\mathcal{B}_{2, \delta}\left(O_{a}, O_{c}\right)$. (cf. Proposition 4.21 and Remark 4.20.) By applying the result of Proposition 4.21, there are then unique elements $\tilde{x}_{k}$ and $\tilde{y}_{k}$ in a $\epsilon / 2$-neighborhood of $x_{k}$ and $y_{k}$, respectively, which satisfy the equations. Notice that these elements $\left(\tilde{x}_{k}, \tilde{y}_{k}\right)$ will in general have $e_{b}^{-}\left(\tilde{x}_{k}\right) \neq e_{b}^{+}\left(\tilde{y}_{k}\right)$, hence they do not define an element in the fibered product. However, we can estimate that the distance between the endpoints goes to zero. In fact, we have

$$
\begin{aligned}
& \left\|e_{b}^{-}\left(\tilde{x}_{k}\right)-e_{b}^{+}\left(\tilde{y}_{k}\right)\right\|_{L_{1}^{2}} \\
& \leq C\left(\operatorname{dist}_{L_{1}^{2}}\left(\lambda_{k}^{-} \tilde{x}_{k}(t), e_{b}^{-}\left(\tilde{x}_{k}\right)\right)+\operatorname{dist}_{L_{1}^{2}}\left(\lambda_{k}^{+} \tilde{y}_{k}(t), e_{b}^{+}\left(\tilde{y}_{k}\right)\right)\right) \\
& \quad+C\left(\left\|\tilde{x}_{k}-x\right\|_{L_{2, \delta}^{2}}+\left\|\tilde{y}_{k}-y\right\|_{L_{2, \delta}^{2}}\right) \\
& \quad+C\left(\operatorname{dist}_{L_{1}^{2}}\left(\lambda^{-} x(t), e_{b}^{-}(x)\right)+\operatorname{dist}_{L_{1}^{2}}\left(\lambda^{+} y(t), e_{b}^{+}(y)\right)\right) .
\end{aligned}
$$

The first two terms and last two terms on the right hand side decay exponentially like $C e^{-\delta|t|}$ by the results of Section 3.4, Theorem 3.12. The gauge 
elements $\lambda_{k}^{-}, \lambda_{k}^{+}, \lambda^{-}$, and $\lambda^{+}$are as in (34), cf. Lemma 4.8. The remaining term is bounded by $\epsilon$.

Since in the statement of Theorem 4.9 we are only interested in the codimension one boundary, we may as well assume that, upon passing to a subsequence, the sequence $\left(\tilde{x}_{k}, \tilde{y}_{k}\right)$ converges in the strong topology to a pair $\left(x^{\prime}, y^{\prime}\right)$. By the result of the previous Claim, we know that this element $\left(x^{\prime}, y^{\prime}\right)$ is in the fibered product $\mathcal{M}\left(O_{a}, O_{b}\right) \times O_{b} \mathcal{M}\left(O_{b}, O_{c}\right)$.

Now consider the pre-glued solutions $x^{\prime} \#_{T_{k}}^{0} y^{\prime}$. By our construction, the original elements $X_{k}$ lie within neighborhoods of radius $\epsilon$ of $x^{\prime} \#_{T_{k}}^{0} y^{\prime}$ in the $L_{2, \delta}^{2}$-norms. By Lemma 6.14, and Remark 4.20 there is a unique zero of the map $f$ in this neighborhood, that is, a unique solution of the SeibergWitten equations, obtained as a small deformation of the approximate solution $x^{\prime} \#_{T_{k}}^{0} y^{\prime}$. This implies the desired equality $X_{k}=x^{\prime} \#_{T_{k}} y^{\prime}$.

This completes the proof of Theorem 4.9.

Before discussing the fine structure of the compactification, we can add a brief comment about the gluing result in the non-equivariant setting, as stated in Lemma 2.16. The difference in the non-equivariant setting is the presence of the extra $U(1)$-gluing parameter, in gluing across the reducible $\theta$. Namely, the gluing map is of the form

$$
\#: \hat{\mathcal{M}}(a, \theta) \times \hat{\mathcal{M}}(\theta, c) \times U(1) \times\left[T_{0}, \infty\right) \rightarrow \hat{\mathcal{M}}(a, c) .
$$

The reason for the presence of the extra $U(1)$ gluing parameter is the fact that the reducible point $\theta$ is not a smooth point in the non-equivariant moduli space, hence, in order to formulate the pre-gluing and gluing construction, it is necessary to lift the pre-gluing to the framed moduli space, in the proximity of the reducible point. More explicitly, we define the pregluing map as

$$
x \#_{T, u}^{0} y= \begin{cases}\lambda_{1}^{+}\left(\mathbb{A}_{1}^{2 T}, \Psi_{1}^{2 T}\right) & t \leq-2 \\ \lambda_{0}\left(A_{0}+\nu, 0\right) & \\ +\exp (i u(t+2))\left(\alpha_{1}(t+2 T), \phi_{1}(t+2 T)\right) & -2 \leq t \leq-1 \\ \lambda_{0}\left(A_{0}+\nu, 0\right)+\exp (i u) . & -1 \leq t \leq 1 \\ \left(\rho^{-}(t)\left(\alpha_{1}(t+2 T), \phi_{1}(t+2 T)\right)\right. & \\ \left.+\rho^{+}(t)\left(\alpha_{2}(t-2 T), \phi_{2}(t-2 T)\right)\right) & \\ \lambda \lambda_{2}^{-}\left(\mathbb{A}_{2}^{-2 T}, \Psi_{2}^{-2 T}\right) & t \geq 1\end{cases}
$$

with the extra gluing parameter $\lambda=\exp (i u)$ in $U(1)$. 
This pre-gluing map gives a choice of a lift of

$$
\hat{\mathcal{M}}(a, \theta) \times \hat{\mathcal{M}}(\theta, c) \times U(1)
$$

to $\hat{\mathcal{M}}\left(O_{a}, \theta\right) \times \hat{\mathcal{M}}\left(\theta, O_{c}\right)$, then the gluing construction works as in the equivariant case and composition with the projection onto the quotient by the $U(1)$ action then provides the resulting gluing map with values in $\hat{\mathcal{M}}(a, c)$.

\subsection{Multiple gluing theorem and corner structure.}

We now generalize the result of Theorem 4.9 of the previous subsection to the case of multiple gluings of broken trajectories in boundary strata of higher codimension. The purpose is to identify the fine structure of the compactification, namely to show that the spaces $\hat{\mathcal{M}}\left(O_{a}, O_{b}\right)$ of flow lines compactify to a $\mathcal{C}^{\infty}$ manifold with corners, in the sense of [44]. We shall follow the notation $\hat{\mathcal{M}}\left(O_{a}, O_{b}\right)^{*}$ to distinguish the compactification from the original space $\hat{\mathcal{M}}\left(O_{a}, O_{b}\right)$.

Theorem 4.23. The compactification $\hat{\mathcal{M}}\left(O_{a}, O_{b}\right)^{*}$ has the structure of a smooth manifold with corners, with codimension $k$ boundary faces of the form

$$
\bigcup_{c_{1}, \cdots c_{k}} \hat{\mathcal{M}}\left(O_{a}, O_{c_{1}}\right)^{*} \times_{O_{c_{1}}} \hat{\mathcal{M}}\left(O_{c_{1}}, O_{c_{2}}\right)^{*} \times \cdots \times{ }_{O_{c_{k}}} \hat{\mathcal{M}}\left(O_{c_{k}}, O_{b}\right)^{*} .
$$

Here the union is over all possible sequences of critical orbits $O_{c_{1}}, \cdots, O_{c_{k}}$ with decreasing indices.

We also have the following.

Corollary 4.24. The endpoint maps $e_{a}^{+}$and $e_{b}^{-}$and their derivatives extend continuously over the boundary and on the boundary they coincide with $e_{a}^{+}$ and $e_{b}^{-}$on $\hat{\mathcal{M}}\left(O_{a}, O_{c_{1}}\right)$ and $\hat{\mathcal{M}}\left(O_{c_{k}}, O_{b}\right)$ respectively. Thus, the maps $e_{a}^{+}$and $e_{b}^{-}$are fibrations with compact fibers in the category of smooth manifolds with corners.

Proof of Theorem 4.23. We proceed as follows. First we prove that $\hat{\mathcal{M}}\left(O_{a}, O_{b}\right)^{*}$ is a $t$-manifold (has a $\mathcal{C}^{\infty}$ structure with corners) in the sense of Definition 1.6.1 of [44]. This amounts to showing that the multiple gluing maps define a compatible set of charts in the sense of Section 1.6 of [44] on open sets in $\hat{\mathcal{M}}\left(O_{a}, O_{b}\right)$ near the boundary. We then show that this $t$ manifold has the structure of a smooth manifold with corners, in the sense of 
Section 1.8 of [44], by showing that the boundary faces satisfy the condition (1.8.7) of [44].

Consider broken trajectories $\left(x_{0}, x_{1}, \ldots, x_{k}\right)$ in a compact subset

$$
\hat{K} \subset \hat{\mathcal{M}}\left(O_{a}, O_{c_{1}}\right) \times{ }_{O_{c_{1}}} \hat{\mathcal{M}}\left(O_{c_{1}}, O_{c_{2}}\right) \times \cdots \times_{O_{c_{k}}} \hat{\mathcal{M}}\left(O_{c_{k}}, O_{b}\right),
$$

where $O_{c_{1}}, \ldots, O_{c_{k}}$ are the critical points with decreasing indices

$$
\mu\left(O_{a}\right)>\mu\left(O_{c_{1}}\right)>\mu\left(O_{c_{2}}\right)>\cdots>\mu\left(O_{c_{k}}\right)>\mu\left(O_{b}\right) .
$$

We introduce multiple gluing maps

$$
\hat{\#}_{i_{1}, \ldots, i_{k}}: \hat{K} \times\left[T_{0}, \infty\right)^{k} \rightarrow \hat{\mathcal{M}}\left(O_{a}, O_{b}\right) .
$$

Here $\left(T_{1}, \ldots, T_{k}\right) \in\left[T_{0}, \infty\right)^{k}$ are gluing parameters, and the indices $\left\{i_{1}, \ldots, i_{k}\right\}$ are a permutation of the set $\{1, \ldots, k\}$ which specifies in which order the multiple gluing is performed as a sequence of $k$ gluings as in Theorem 4.9. For instance, we have

$$
\hat{\#}_{1,2, \ldots, k}^{T_{1}, \ldots, T_{k}}\left(x_{0}, x_{1}, \ldots, x_{k}\right)=\left(\cdots\left(\left(\left(x_{0} \hat{\#}_{T_{1}} x_{1}\right) \hat{\#}^{T_{2}} x_{2}\right) \hat{\#}_{T_{3}} x_{4}\right) \cdots\right) \hat{\#}_{T_{k}} x_{k}
$$

or

$$
\left.\hat{\#}_{2,1,3, \ldots, k}^{T_{1}, \ldots, T_{k}}\left(x_{0}, x_{1}, \ldots, x_{k}\right)=\left(\cdots\left(x_{0} \hat{\#}_{T_{1}}\left(x_{1} \hat{\#}^{T_{2}} x_{2}\right)\right) \hat{\#}_{T_{3}} x_{3}\right) \cdots\right) \hat{\#}_{T_{k}} x_{k}
$$

etc.

The gluing construction is non-canonical, in the sense that the identification of the normal bundle $\mathcal{T}^{\perp}$ in the pre-gluing construction, as in Lemma 4.13 , is dependent on the order of the gluing, hence there is no obvious associativity law for multiple gluings. However, we are going to show that the maps $\hat{\#}_{i_{1}, \ldots, i_{k}}^{T_{1}, \ldots, T_{k}}$, or rather their inverses, to be consistent with the notation of (1.6.1) of [44], define a system of charts of a $\mathcal{C}^{\infty}$ structure with corners, or $t$-manifold. We prove the following result, which ensures that the $\hat{\#}_{i_{1}, \ldots, i_{k}}^{T_{1}, \ldots, T_{k}}$ are local diffeomorphisms as needed.

Proposition 4.25. Suppose given $O_{a}, O_{c_{1}}, \cdots O_{c_{k}}$ and $O_{b}$ in $\mathcal{M}^{0}$ with decreasing indices. Then, given a compact set $\hat{K}$ in

$$
\hat{\mathcal{M}}\left(O_{a}, O_{c_{1}}\right) \times_{O_{c_{1}}} \hat{\mathcal{M}}\left(O_{c_{1}}, O_{c_{2}}\right) \times \cdots \times_{O_{c_{k}}} \hat{\mathcal{M}}\left(O_{c_{k}}, O_{b}\right),
$$

there is a lower bound $T_{0}(K)>0$ such that the gluing maps $\hat{\#}_{i_{1}, \ldots, i_{k}}^{T_{1}, \ldots, T_{k}}$ define local diffeomorphisms

$$
\hat{\#}_{i_{1}, \ldots, i_{k}}: \quad \hat{K} \times\left[T_{0}(K), \infty\right)^{k} \longrightarrow \hat{\mathcal{M}}\left(O_{a}, O_{b}\right) .
$$


where the orientation on the left hand side is the one induced by the product orientation on

$$
\left(\left(\left(\hat{\mathcal{M}}\left(O_{a}, O_{c_{1}}\right) \times \mathbb{R} \times \hat{\mathcal{M}}\left(O_{c_{1}}, O_{c_{2}}\right)\right) \times \mathbb{R}\right) \times \cdots \times \mathbb{R}\right) \times \hat{\mathcal{M}}\left(O_{c_{k}}, O_{b}\right) .
$$

Proof. We want to generalize the analogous statement proved in Theorem 4.9 for the case of one intermediate critical point.

First, we need to show the analogue of the error estimate (56), which shows that the glued solutions $\#_{i_{1}, \ldots, i_{k}}^{T_{1}, \ldots, T_{k}}\left(x_{0}, \ldots, x_{k}\right)$ converge to broken trajectories in the limit when a certain subset $\left\{T_{i_{1}}, \ldots T_{i_{m}}\right\}$ of the gluing parameters satisfies $T_{i_{\ell}} \rightarrow \infty$.

We then need to show that a sequence of solutions in $\hat{\mathcal{M}}\left(O_{a}, O_{b}\right)$ that converges to a broken trajectory $\left(x_{0}, \ldots, x_{k}\right)$ in the boundary

$$
\hat{\mathcal{M}}\left(O_{a}, O_{c_{1}}\right) \times_{O_{c_{1}}} \cdots \times_{O_{c_{k}}} \hat{\mathcal{M}}\left(O_{c_{k}}, O_{b}\right)
$$

lies eventually in the range of a gluing map $\#_{i_{1}, \ldots, i_{k}}^{T_{1}, \ldots, T_{k}}$. We first prove that, when the gluing parameters $\left\{T_{1}, \ldots, T_{k}\right\}$ satisfy $T_{i} \rightarrow \infty$, for all $i=1, \ldots, k$, we have an estimate

$$
\left\|\sigma_{k}\left(x_{0}, \ldots, x_{k}, T_{1}, \ldots, T_{k}\right)\right\|_{L_{1, \delta}^{2}} \leq C e^{-\delta T},
$$

with $T=\min \left\{T_{1}, \ldots, T_{k}\right\}$. This is the analogue of the estimate (103) for multiple gluing. Here $\sigma_{k}$ is the error term

$$
\sigma_{k}\left(x_{0}, \ldots, x_{k}, T_{1}, \ldots, T_{k}\right)=\#_{i_{1}, \ldots, i_{k}}^{T_{1}, \ldots, T_{k}}\left(x_{0}, \ldots, x_{k}\right)-\#_{i_{1}, \ldots, i_{k}}^{0 T_{1}, \ldots, T_{k}}\left(x_{0}, \ldots, x_{k}\right),
$$

with $\#_{i_{1}, \ldots, i_{k}}^{0 T_{1}, \ldots, T_{k}}$ the pre-gluing map, namely the composite of $k$ pre-gluing maps as in $(35)$, in the order specified by $\left(i_{1}, \ldots, i_{k}\right)$.

We prove the estimate (64) for the case of the gluing map $\#_{1, \ldots, k}^{T_{1}, \ldots, T_{k}}$. The same procedure works with a permutation of the order of gluing. We proceed by induction on $k$. The case $k=1$ follows from Theorem 4.9. Assume as induction hypothesis that the gluing map $\#_{1, \ldots, k-1}^{T_{1}, \ldots, T_{k-1}}$ can be written as

$$
\begin{aligned}
& \#_{1, \ldots, k-1}^{T_{1}, \ldots, T_{k-1}}\left(x_{0}, \ldots, x_{k-1}\right) \\
& =\#^{T_{1}, \ldots, T_{k-1}}\left(x_{0}, \ldots, x_{k-1}\right)+\sigma_{k-1}\left(x_{0}, \ldots, x_{k-1}, T_{1}, \ldots, T_{k-1}\right)
\end{aligned}
$$


with $\#_{1, \ldots, k-1}^{0 T_{1}, \ldots, T_{k-1}}$ the pre-gluing map (i.e., the composition of $k-1$ pre-gluing maps (35), and with the error term $\sigma_{k-1}$ satisfying the estimate

$$
\left\|\sigma_{k-1}\left(x_{0}, \ldots, x_{k-1}, T_{1}, \ldots, T_{k-1}\right)\right\|_{L_{1, \delta}^{2}} \leq C e^{-\delta T},
$$

with $T=\min \left\{T_{1}, \ldots, T_{k_{1}}\right\}$. We can then form the pre-glued solution $y \#_{T_{k}}^{0} x_{k}$, with

$$
y=\#_{1, \ldots, k-1}^{T_{1}, \ldots, T_{k-1}}\left(x_{0}, \ldots, x_{k-1}\right) .
$$

By Proposition 4.21 this approximate solution can be deformed to an actual solution $y \#_{T_{k}} x_{k}$, which by definition is the same as

$$
y \#_{T_{k}} x_{k}=\#_{1, \ldots, k}^{T_{1}, \ldots, T_{k}}\left(x_{0}, \ldots, x_{k}\right) .
$$

We need to estimate the difference $y \#_{T_{k}} x_{k}-y_{0} \#_{T_{k}}^{0} x_{k}$, where $y_{0}$ is the approximate solution

$$
y_{0}=\#_{1, \ldots, k-1}^{0 T_{1}, \ldots, T_{k-1}}\left(x_{0}, \ldots, x_{k-1}\right)
$$

We have

(65) $\left\|y \#_{T_{k}} x_{k}-y_{0} \#_{T_{k}}^{0} x_{k}\right\|_{L_{1, \delta}^{2}}$

$$
\leq\left\|y \#_{T_{k}} x_{k}-y \#_{T_{k}}^{0} x_{k}\right\|_{L_{1, \delta}^{2}}+\left\|y \#_{T_{k}}^{0} x_{k}-y_{0} \#_{T_{k}}^{0} x_{k}\right\|_{L_{1, \delta}^{2}} .
$$

Now we can estimate the second term on the right hand side by

$$
\left\|y \#_{T_{k}}^{0} x_{k}-y_{0} \#_{T_{k}}^{0} x_{k}\right\|_{L_{1, \delta}^{2}} \leq\left\|F_{\#_{T_{k}}}\right\| \cdot\left\|y-y_{0}\right\|_{L_{1, \delta}^{2}} .
$$

We know that (44) has norm bounded by $C e^{-\delta T_{k}}$. Moreover, the quantity $\left\|y-y_{0}\right\|$ is given by

$$
\left\|\sigma_{k-1}\left(x_{0}, \ldots, x_{k-1}, T_{1}, \ldots, T_{k-1}\right)\right\|_{L_{1, \delta}^{2}} .
$$

Using the induction hypothesis, we obtain

$$
\left\|y \#_{T_{k}}^{0} x_{k}-y_{0} \#_{T_{k}}^{0} x_{k}\right\|_{L_{1, \delta}^{2}} \leq C e^{-\delta T} .
$$

The first term in the right hand side of (65), on the other hand, is given by

$$
\left\|y \#_{T_{k}} x_{k}-y \#_{T_{k}}^{0} x_{k}\right\|_{L_{1, \delta}^{2}}=\left\|\sigma\left(y, x_{k}, T_{k}\right)\right\|_{L_{1, \delta}^{2}} .
$$


By the estimate (103) (cf. Proposition 4.21, Lemma 6.14, Remark 4.20, and Proposition 4.21), this satisfies the estimate

$$
\left\|\sigma\left(y, x_{k}, T_{k}\right)\right\|_{L_{1, \delta}^{2}} \leq C e^{-\delta T_{k}} .
$$

Thus, we have the next step of the induction, namely we have obtained

$$
\left\|\sigma_{k}\left(x_{0}, \ldots, x_{k}, T_{1}, \ldots, T_{k}\right)\right\|_{L_{1, \delta}^{2}} \leq C e^{-\delta \cdot \min \left\{T, T_{k}\right\}}
$$

for

$$
\sigma_{k}\left(x_{0}, \ldots, x_{k}, T_{1}, \ldots, T_{k}\right)=y \#_{T_{k}} x_{k}-y_{0} \#_{T_{k}}^{0} x_{k} .
$$

Now consider a sequence $X_{\ell}$ in $\mathcal{M}\left(O_{a}, O_{b}\right)$ that converges smoothly on compact sets, in the sense of Theorem 4.9 , to a broken trajectory $\left(x_{0}, \ldots, x_{k}\right)$ in the fibered product

$$
\mathcal{M}\left(O_{a}, O_{c_{1}}\right) \times{ }_{O_{c_{1}}} \cdots \times{ }_{O_{c_{k}}} \mathcal{M}\left(O_{c_{k}}, O_{b}\right) .
$$

We need to show that these $X_{\ell}$ belong eventually to the range of at least one of the multiple gluing maps $\#_{i_{1}, \cdots, i_{k}}$. We proceed as in the analogous argument in Theorem 4.9, for a single gluing. Convergence in the sense of Theorem 4.9 implies that there exist time shifts $\left\{T_{1, \ell}, \ldots, T_{k, \ell}\right\}$ with $T_{i, \ell} \rightarrow$ $\infty$ for $i=1, \ldots k$, and combinatorial data which describe the order in which successive limits on compact sets and reparameterizations are considered in Theorem 4.9. These data determine which gluing maps $\#_{i_{1}, \cdots, i_{k}}$ have the points $X_{\ell}$ in the range. These combinatorial data can be described as the set of binary trees with $k+1$ leaves, one root, and $k-1$ intermediate nodes. To one such tree we associate a cutoff element

$$
\left(\eta_{0}^{T_{1, \ell}, \ldots, T_{k, \ell}}\left(X_{\ell}\right), \ldots, \eta_{\ell}^{T_{1, \ell}, \ldots, T_{k, \ell}}\left(X_{\ell}\right)\right)
$$

where the $\eta_{i}^{T_{1, \ell}, \ldots, T_{k, \ell}}$ act as a composition of time shifts and cutoff functions, as specified by the combinatorics of the tree.
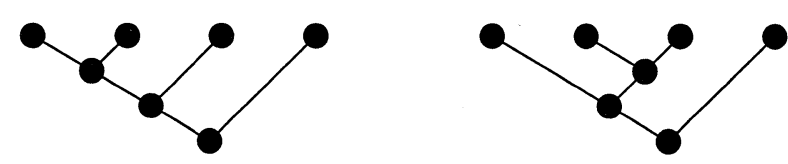
For instance, the two trees of the figure correspond respectively to the elements

$$
\begin{aligned}
& \left(\rho_{1-T_{1, \ell}}^{-}\left(\rho_{1-T_{2, \ell}}^{-}\left(\rho_{1-T_{3, \ell}}^{-} X_{\ell}^{-T_{3, \ell}}\right)^{-T_{2, \ell}}\right)^{-T_{1, \ell}},\right. \\
& \rho_{-1+T_{1, \ell}}^{+}\left(\rho_{1-T_{2, \ell}}^{-}\left(\rho_{1-T_{3, \ell}}^{-} X_{\ell}^{-T_{3, \ell}}\right)^{-T_{2, \ell}}\right)^{T_{1, \ell}} \\
& \rho_{-1+T_{2, \ell}}^{+}\left(\rho_{1-T_{3, \ell}}^{-} X_{\ell}^{-T_{3, \ell}}\right)^{T_{2, \ell}} \\
& \left.\rho_{-1+T_{3, \ell}}^{+} X_{\ell}^{T_{3, \ell}}\right)
\end{aligned}
$$

and

$$
\begin{aligned}
& \left(\rho_{1-T_{1, \ell}}^{-}\left(\rho_{1-T_{3, \ell}}^{-} X_{\ell}^{-T_{3, \ell}}\right)^{-T_{1, \ell},}\right. \\
& \rho_{1-T_{1, \ell}}^{-}\left(\rho_{-1+T_{2, \ell}}^{+}\left(\rho_{1-T_{3, \ell}}^{-} X_{\ell}^{-T_{3, \ell}}\right)^{T_{2, \ell}}\right)^{-T_{1, \ell},}, \\
& \rho_{-1+T_{1, \ell}}^{+}\left(\rho_{-1+T_{2, \ell}}^{+}\left(\rho_{1-T_{3, \ell}}^{-} X_{\ell}^{-T_{3, \ell}}\right)^{T_{2, \ell}}\right)^{T_{1, \ell}}, \\
& \left.\rho_{-1+T_{3, \ell}}^{+} X_{\ell}^{T_{3, \ell}}\right) .
\end{aligned}
$$

These correspond to cases where a sequence of solutions $X_{\ell}$ in, say, $\mathcal{M}\left(O_{a}, O_{b}\right)$ converges smoothly on compact sets, after the different reparameterizations specified above, to elements in $\mathcal{M}\left(O_{a}, O_{c_{1}}\right), \mathcal{M}\left(O_{c_{1}}, O_{c_{2}}\right)$, $\mathcal{M}\left(O_{c_{2}}, O_{c_{3}}\right)$, and $\mathcal{M}\left(O_{c_{3}}, O_{b}\right)$, respectively.

We denote by $\left(x_{1, \ell}, \ldots, x_{k, \ell}\right)$ the projection onto the slices of the cutoff elements (66). The elements $x_{i, \ell}$ are no longer solutions of the Seiberg-Witten equations, however, by hypothesis, for $\ell$ large enough, they are contained in $\epsilon / 2^{k+1}$-neighborhoods of the solutions $x_{i}$, for $i=0, \ldots, k$. Pick $\epsilon$ satisfying

$$
\epsilon \leq \max _{0 \leq j \leq k+1}\left\{\epsilon_{c_{j} c_{j+1}}\right\}
$$

where the $\epsilon_{c_{j} c_{j+1}}$ is the constant for the contraction principle, as in Lemma 6.14 in $\mathcal{B}_{2, \delta}\left(O_{c_{j}}, O_{c_{j+1}}\right)$, and we take $c_{0}=a$ and $c_{k+1}=b$. Then, proceeding as in the proof of Theorem 4.9 , we find elements $\tilde{x}_{i, \ell}$, for $i=0, \ldots k$ that satisfy the Seiberg-Witten equations and are contained in $\epsilon / 2^{k}$-neighborhoods of the elements $x_{i}$. The elements $\left(\tilde{x}_{0, \ell}, \ldots, \tilde{x}_{k, \ell}\right)$ are not necessarily in the fibered product, however, by the same argument used in the proof of Theorem 4.9 , we obtain convergence of the elements $\left(\tilde{x}_{0, \ell}, \ldots, \tilde{x}_{k, \ell}\right)$ to a limit $\left(x_{0}^{\prime}, \ldots, x_{k}^{\prime}\right)$ in the fibered product

$$
\mathcal{M}\left(O_{a}, O_{c_{1}}\right) \times_{O_{c_{1}}} \cdots \times_{O_{c_{k}}} \mathcal{M}\left(O_{c_{k}}, O_{b}\right) .
$$

By construction, the approximate solution

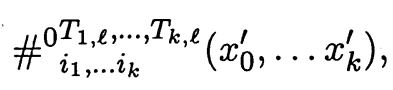


with the order $\left(i_{1}, \ldots i_{k}\right)$ of gluing specified by the combinatorial data, is contained in an $\epsilon$-neighborhood of $X_{\ell}$ in the $L_{2, \delta}^{2}$-norm. By the contraction argument of Lemma 6.14 together with the first part of the proof of this Proposition, we know that we must then have

$$
X_{\ell}=\#_{i_{1}, \ldots i_{k}}^{T_{1, \ell}, \ldots, T_{k, \ell}}\left(x_{0}^{\prime}, \ldots, x_{k}^{\prime}\right) .
$$

This completes the proof.

Thus, the changes of coordinates

$$
\hat{\#}_{i_{1}, \ldots, i_{k}}^{T_{1}, \ldots, T_{k}} \circ\left(\hat{\#}_{j_{1}, \ldots, j_{\ell}}^{\tau_{1}, \ldots, \tau_{\ell}}\right)^{-1}
$$

are local diffeomorphisms between open subsets of

$$
\mathbb{R}_{k}^{n}=\mathbb{R}^{n-k} \times\left[T_{0}, \infty\right)^{k}
$$

and

$$
\mathbb{R}_{\ell}^{n}=\mathbb{R}^{n-\ell} \times\left[T_{0}, \infty\right)^{\ell},
$$

with $n=\operatorname{dim} \hat{\mathcal{M}}\left(O_{a}, O_{b}\right)$, as prescribed in Section 1.6 of [44].

Notice, in particular, that given two multiple gluing maps $\#_{i_{1}, \ldots, i_{k}}^{T_{1}, \ldots, T_{k}}$ and $\#_{j_{1}, \ldots, j_{k}}^{T_{1}, \ldots, T_{k}}$, for two different orders of gluing $\left(i_{1}, \ldots, i_{k}\right) \neq\left(j_{1}, \ldots j_{k}\right)$, we obtain an estimate

$$
\left\|\#_{i_{1}, \ldots, i_{k}}^{T_{1}, \ldots, T_{k}}\left(x_{0}, \ldots, x_{k}\right)-\#_{j_{1}, \ldots j_{k}}^{T_{1}, \ldots, T_{k}}\left(x_{0}, \ldots, x_{k}\right)\right\|_{L_{1, \delta}^{2}} \leq C e^{-\delta T}
$$

for $T=\min \left\{T_{1}, \ldots, T_{k}\right\}$. The proof of this estimate is completely analogous to the argument used in the proof of Proposition 4.25. For instance, we can estimate

$$
\begin{aligned}
& \left\|\left(x_{0} \#_{T_{1}} x_{1}\right) \#_{T_{2}} x_{2}-x_{0} \#_{T_{1}}\left(x_{1} \#_{T_{2}} x_{2}\right)\right\| \\
& \leq \\
& \quad\left\|\left(x_{0} \#_{T_{1}} x_{1}\right) \#_{T_{2}} x_{2}-\left(x_{0} \#_{T_{1}} x_{1}\right) \#_{T_{2}}^{0} x_{2}\right\| \\
& \quad+\left\|\left(x_{0} \#_{T_{1}} x_{1}\right) \#_{T_{2}}^{0} x_{2}-x_{0} \#_{T_{1}}^{0} x_{1} \#_{T_{2}}^{0} x_{2}\right\| \\
& \quad+\left\|x_{0} \#_{T_{1}}^{0} x_{1} \#_{T_{2}}^{0} x_{2}-x_{0} \#_{T_{1}}^{0}\left(x_{1} \#_{T_{2}} x_{2}\right)\right\| \\
& \quad+\left\|x_{0} \#_{T_{1}}^{0}\left(x_{1} \#_{T_{2}} x_{2}\right)-x_{0} \#_{T_{1}}\left(x_{1} \#_{T_{2}} x_{2}\right)\right\| \\
& \leq C e^{-\delta T_{2}}+C e^{-\delta T_{1}+T_{2}}+C e^{-\delta T_{1}+T_{2}}+C e^{-\delta T_{1}} .
\end{aligned}
$$

The estimate (67) implies that, when all the $T_{i}$ go to infinity, the glued elements $\#_{i_{1}, \ldots, i_{k}}^{T_{1}, \ldots, T_{k}}\left(x_{0}, \ldots, x_{k}\right)$ and $\#_{j_{1}, \ldots j_{k}}^{T_{1}, \ldots, T_{k}}\left(x_{0}, \ldots, x_{k}\right)$ end up in the same 
coordinate patch. This may not be the case if only some of the $T_{i}$ tend to infinity and other remain bounded.

We have shown, as a result of Proposition 4.25, and by Lemma 1.7.1 of [44], that the strata

$$
\hat{\mathcal{M}}\left(O_{a}, O_{c_{1}}\right)^{*} \times_{O_{c_{1}}} \times \cdots \times{ }_{O_{c_{k}}} \hat{\mathcal{M}}\left(O_{c_{k}}, O_{b}\right)^{*}
$$

inherit the structure of smooth $t$-sub-manifolds of codimension $k$ in $\hat{\mathcal{M}}\left(O_{a}, O_{b}\right)^{*}$.

The codimension one boundary strata described in Theorem 4.8, compactified to smooth $t$-manifolds, define the boundary hypersurfaces of $\hat{\mathcal{M}}\left(O_{a}, O_{b}\right)^{*}$, as in (1.8.3) and (1.8.4) of [44].

Let us recall that a $\mathcal{C}^{\infty}$ manifold with corners is a $t$-manifold where all the boundary faces are $\mathcal{C}^{\infty}$ embedded sub-manifolds. According to Section 1.8 of [44], to ensure that this is the case, namely that the compactification $\hat{\mathcal{M}}\left(O_{a}, O_{b}\right)^{*}$ has the structure of a $\mathcal{C}^{\infty}$ manifold with corners, one only needs to check the following fact.

Lemma 4.26. Every compactified codimension $k$ boundary face

$$
\hat{\mathcal{M}}\left(O_{a}, O_{c_{1}}\right)^{*} \times O_{c_{1}} \times \cdots \times{ }_{O_{c_{k}}} \hat{\mathcal{M}}\left(O_{c_{k}}, O_{b}\right)^{*}
$$

of $\hat{\mathcal{M}}\left(O_{a}, O_{b}\right)^{*}$ is a component of (precisely) one intersection of $k$ boundary hypersurfaces

$$
H_{i_{1}} \cap \ldots \cap H_{i_{k}} .
$$

Proof of Lemma 4.26. All the boundary hypersurfaces are identified by Theorem 4.9. Thus, we see that the Lemma is verified by setting

$$
\begin{array}{ll}
H_{i_{1}}= & \hat{\mathcal{M}}\left(O_{a}, O_{c_{1}}\right)^{*} \times O_{c_{1}} \hat{\mathcal{M}}\left(O_{c_{1}}, O_{b}\right)^{*} \\
H_{i_{2}}= & \hat{\mathcal{M}}\left(O_{a}, O_{c_{2}}\right)^{*} \times{ }_{O_{c_{2}}} \hat{\mathcal{M}}\left(O_{c_{2}}, O_{b}\right)^{*} \\
\vdots & \vdots \\
H_{i_{k-1}}= & \hat{\mathcal{M}}\left(O_{a}, O_{c_{k-1}}\right)^{*} \times{ }_{O_{c_{k-1}}} \hat{\mathcal{M}}\left(O_{c_{k-1}}, O_{b}\right)^{*} \\
H_{i_{k}}= & \hat{\mathcal{M}}\left(O_{a}, O_{c_{k}}\right)^{*} \times{ }_{O_{c_{k}}} \hat{\mathcal{M}}\left(O_{c_{k}}, O_{b}\right)^{*} .
\end{array}
$$

This completes the proof of Theorem 4.23. 
Corollary 4.24 now follows, by considering the restriction of the asymptotic value maps $e_{a}^{+}$and $e_{b}^{-}$to the range of the gluing maps. By the convergence property, when a subset of the gluing parameters goes to infinity, we obtain that the asymptotic value maps restrict to the corresponding maps on the boundary strata.

\section{Equivariant Homology.}

Let us recall briefly the construction of the de Rham model for $U(1)$ equivariant cohomology (and homology) on a finite dimensional manifold with a $U(1)$ action. The main reference is [2].

Let $W$ be the Weil algebra of the Lie algebra $i \mathbb{R}$ of $U(1)$. This is a free commutative graded algebra in one generator $\theta$ of degree 1 and one generator $\Omega$ of degree 2 , with differential $\delta$ that satisfies

$$
\delta \theta=\Omega \quad \delta \Omega=0 .
$$

Let $M$ be a manifold with a $U(1)$ action. Consider the complex

$$
C^{*}=W \otimes \Omega^{*}(M)
$$

with differential

$$
d_{U(1)}=d-\Omega c(T) .
$$

Here $T$ is the vector field on $M$ generated by the infinitesimal $U(1)$ action and $c$ is the unique derivation in $W$ that satisfies

$$
c(\theta)=1 \quad c(\Omega)=0 .
$$

We choose a sub-complex of $C^{*}$ by taking the cochains on which $c+c(T)$ and $\mathcal{L}(T)$ vanish, where $\mathcal{L}(T)$ is the Lie derivative. Let us call this subcomplex $\Omega_{U(1)}^{*}(M)$. An alternative description of the complex $\Omega_{U(1)}^{*}(M)$ is

$$
\Omega_{U(1)}^{*}(M)=\mathbb{R}[\Omega] \otimes \Omega_{0}^{*}(M)
$$

where $\Omega_{0}^{*}(M)$ are de Rham forms that are annihilated by the Lie differentiation $\mathcal{L}(T)$. The cohomology $H^{*}\left(\Omega_{U(1)}^{*}(M), d_{U(1)}\right)$ is isomorphic to the equivariant cohomology with real coefficients,

$$
H^{*}\left(\Omega_{U(1)}^{*}(M), d_{U(1)}\right) \cong H_{U(1)}^{*}(M, \mathbb{R}) .
$$

In order to compute equivariant homology with real coefficients a de Rham complex can be constructed as in [8] by considering differential forms graded by the dimension of $M$ minus the degree,

$$
\Omega_{* U(1)}(M)=\mathbb{R}[\Omega] \otimes \Omega_{0}^{\operatorname{dim}(M)-*}(M),
$$


with boundary operator $\partial_{U(1)}=d-c(\theta) \otimes c(T)$.

Austin and Braam [8] proved that this complex computes the same homology as the equivariant complex of currents introduced by Duflo and Vergne [19] and studied by Kumar and Vergne [32]. With this understood, we can consider the complex $\Omega_{* U(1)}\left(O_{a}\right)$ associated to each critical orbit $O_{a}$ in $\mathcal{M}_{0}$. This will be a copy of the polynomial algebra $\mathbb{R}[\Omega]$ for the fixed point and a complex of $\mathbb{R}[\Omega]$-modules with de Rham forms in degree zero and one in the case of orbits that come from irreducibles.

We can give a more explicit description of the boundary operator $\partial_{U(1)}$ in this case. Let $O_{a}$ be a critical orbit with a free $U(1)$ action. Then, the generators of $\Omega_{0}^{1-*}\left(O_{a}\right)$ are a 1-form $\eta_{a}$ that generates $H^{1}\left(S^{1}\right)$, in degree zero, and a zero-form $1_{a}$ (the constant function equal to one on $O_{a}$ ), in degree one. Thus we have

$$
\partial_{U(1)}\left(\Omega^{n} \otimes 1_{a}\right)=0
$$

and

$$
\partial_{U(1)}\left(\Omega^{n} \otimes \eta_{a}\right)=-\Omega^{n-1} \otimes 1_{a} .
$$

\subsection{The equivariant complex.}

We can form the bigraded complex that computes equivariant Floer homology as in [6], defined by

$$
C_{k U(1)}(Y)=\bigoplus_{\mu\left(O_{a}\right)=i, i+j=k} \Omega_{j U(1)}\left(O_{a}\right),
$$

with differentials

$$
D_{a, b} \eta=\left\{\begin{array}{lr}
\partial_{U(1)} \eta & O_{a}=O_{b} \\
(-1)^{1-r(\eta)}\left(e_{b}^{-}\right)_{*}\left(e_{a}^{+}\right)^{*} \eta & \mu\left(O_{a}\right)>\mu\left(O_{b}\right) \\
0 & \text { otherwise }
\end{array}\right.
$$

Here $\eta$ is an equivariant differential form on the orbit $O_{a}$, that is, an element of $\Omega_{* U(1)}\left(O_{a}\right)$. The number $r(\eta)$ is the de Rham degree of $\eta$, that is, the maximum degree of the elements of $\Omega_{0}^{*}\left(O_{a}\right)$ that appear in the expression of $\eta$. Recall that $\Omega_{* U(1)}\left(O_{a}\right)$ is the dual of the de Rham complex, and the forms are graded by $\operatorname{dim}\left(O_{a}\right)-*=1-*$.

The analogous complex that computes equivariant Floer cohomology is given by

$$
C^{k}{ }_{U(1)}(Y)=\bigoplus_{\mu\left(O_{a}\right)=i, i+j=k} \Omega^{j}{ }_{U(1)}\left(O_{a}\right)
$$


with coboundaries

$$
\delta_{a, b} \eta=\left\{\begin{array}{lr}
d_{U(1)} \eta & O_{a}=O_{b} \\
(-1)^{r(\eta)}\left(e_{a}^{+}\right)_{*}\left(e_{b}^{-}\right)^{*} \eta & \mu\left(O_{a}\right)>\mu\left(O_{b}\right) \\
0 & \text { otherwise }
\end{array}\right.
$$

Here $\eta$ is an equivariant form, that is an element of $O_{b}$ and $r(\eta)$ is the de Rham degree of $\eta$, i.e. the maximum degree of the elements of $\Omega_{0}^{*}\left(O_{b}\right)$ that appear in the expression of $\eta$.

The boundary map is well defined, since the endpoint maps are compatible with the boundary strata as stated in 4.24. Notice that, as pointed out in [6], for dimensional reasons the maps $D_{a, b}$ are trivial whenever $\mu\left(O_{a}\right) \geq \mu\left(O_{b}\right)+3$. A more explicit description of the boundary map will be given in the following.

\subsection{Equivariant Floer Homology.}

We can prove that the composites $D^{2}$ and $\delta^{2}$ are zero. Moreover we can see that there is a duality at the level of forms that induces a duality between equivariant Floer homology and cohomology as in [6] and [8].

Theorem 5.1. The composite maps $D^{2}$ and $\delta^{2}$ are zero, this means that the identities

$$
\begin{gathered}
D_{a, c}^{2}=\sum_{b} D_{a, b} D_{b, c}=0 \\
\delta_{a, c}^{2}=\sum_{b} \delta_{b, c} \delta_{a, b}=0
\end{gathered}
$$

hold, with $O_{b}$ that ranges among critical orbits satisfying $\mu\left(O_{a}\right) \geq \mu\left(O_{b}\right) \geq$ $\mu\left(O_{c}\right)$. Moreover there is a pairing $\langle$,$\rangle of forms in C_{* U(1)}$ and in $C^{*}{ }_{U(1)}$ that satisfies

$$
\langle D \eta, \gamma\rangle=\langle\eta, \delta \gamma\rangle
$$

Proof. The statement is true for $O_{a}=O_{c}$. Given critical orbits with $\mu\left(O_{a}\right)>$ $\mu\left(O_{c}\right)$, we have the expression

$$
\begin{gathered}
D_{a, c}^{2} \eta=(-1)^{r(\eta)} \sum_{\left\{O_{b} \mid \mu\left(O_{a}\right)>\mu\left(O_{b}\right)>\mu\left(O_{c}\right)\right\}}(-1)^{r\left(\left(e_{b}^{-}\right)_{*}\left(e_{a}^{+}\right)^{*} \eta\right)} \\
\left(e_{c}^{-}\right)_{*}\left(e_{b}^{+}\right)^{*}\left(e_{b}^{-}\right)_{*}\left(e_{a}^{+}\right)^{*} \eta+\left(e_{c}^{-}\right)_{*}\left(e_{a}^{+}\right)^{*} \partial_{U(1)} \eta+\partial_{U(1)}\left(e_{c}^{-}\right)_{*}\left(e_{a}^{+}\right)^{*} \eta .
\end{gathered}
$$


This expression vanishes since the co-dimension 1 boundary of $\hat{\mathcal{M}}\left(O_{a}, O_{b}\right)^{*}$ is the union of the components $\hat{\mathcal{M}}\left(O_{a}, O_{b}\right)^{*} \times O_{b} \hat{\mathcal{M}}\left(O_{b}, O_{c}\right)^{*}$, as in 4.9. Thus, if we use Stokes theorem applied to the fibration with boundary $e_{c}^{-}: \hat{\mathcal{M}}\left(O_{a}, O_{c}\right)^{*} \rightarrow O_{c}$, we obtain [6] that

$$
\partial_{U(1)}\left(e_{c}^{-}\right)_{*} \gamma=\left(e_{c}^{-}\right)_{*} \partial_{U(1)} \gamma-(-1)^{r(\gamma)-\operatorname{dim} F}\left(e_{c}^{-}\right)_{\partial, *} \gamma
$$

Here $\gamma$ is an equivariant form on $\hat{\mathcal{M}}\left(O_{a}, O_{c}\right)^{*}$ and the map $\left(e_{c}^{-}\right)_{\partial, *}$ is the push-forward map induced by the restriction of the bundle to the boundary of each fiber $F$. If we choose $\gamma$ of the form $\gamma=\left(e_{a}^{+}\right)^{*} \eta$ where $\eta$ is an equivariant form on the orbit $O_{a}$, then the map $\left(e_{c}^{-}\right)_{\partial, *}$ can be written as

$$
\left(e_{c}^{-}\right)_{\partial, *} \gamma=\sum_{b}\left(e_{c}^{-}\right)_{*}\left(e_{b}^{+}\right)^{*}\left(e_{b}^{-}\right)_{*}\left(e_{a}^{+}\right)^{*} \eta,
$$

for all the $b$ that satisfy $\mu\left(O_{a}\right)>\mu\left(O_{b}\right)>\mu\left(O_{c}\right)$, as shown in the diagram. Notice that the sign $(-1)^{r\left(\left(e_{b}^{-}\right)_{*}\left(e_{a}^{+}\right)^{*} \eta\right)}$ is exactly the sign $(-1)^{r\left(\left(e_{a}^{+}\right)^{*} \eta\right)-\operatorname{dim} F}$.

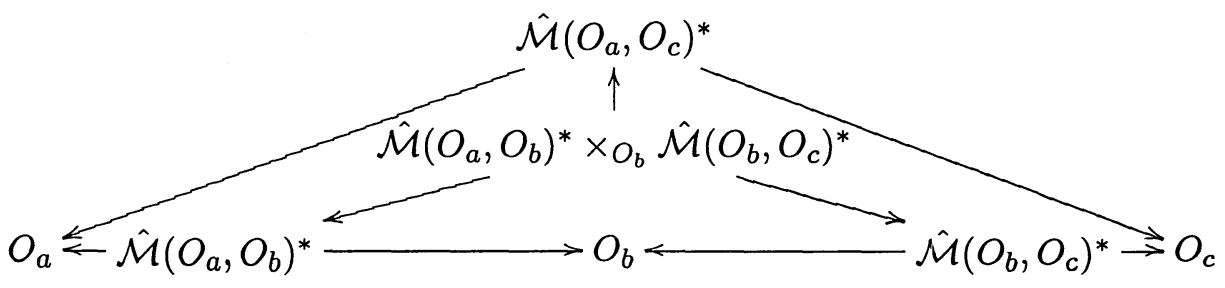

The statement about the pairing follows from the identity

$$
\int_{O_{b}}\left(e_{b}^{-}\right)_{*}\left(e_{a}^{+}\right)^{*} \eta \wedge \gamma=\int_{\hat{\mathcal{M}}\left(O_{a}, O_{b}\right)^{*}}\left(e_{a}^{+}\right)^{*} \eta \wedge\left(e_{b}^{-}\right)^{*} \gamma .
$$

Definition 5.2. We define the equivariant Floer homology and cohomology to be

$$
H F_{*}^{S W}{ }_{U(1)}=H_{*}\left(C_{* U(1)}, D\right)
$$

and

$$
H F^{S W^{*}}{ }^{*}=H^{*}\left(C_{U(1)}^{*}, \delta\right) .
$$

We now give a more detailed description of the equivariant Floer complex, which will be useful in Section 6 and Section 7 . 
Proposition 5.3. Let $\Omega^{n} \otimes \eta_{a}$ and $\Omega^{n} \otimes 1_{a}$ be the generators of the equivariant Floer complex. The only possibly non-trivial coefficients of the boundary map $D$ are those of the form

$$
\left\langle\Omega^{n-1} \otimes 1_{a}, D\left(\Omega^{n} \otimes \eta_{a}\right)\right\rangle,
$$

or of the form

$$
\begin{aligned}
& \left\langle\Omega^{n} \otimes 1_{b}, D\left(\Omega^{n} \otimes 1_{a}\right)\right\rangle, \\
& \left\langle\Omega^{n} \otimes \eta_{b}, D\left(\Omega^{n} \otimes \eta_{a}\right)\right\rangle,
\end{aligned}
$$

when $\mu\left(O_{a}\right)-\mu\left(O_{b}\right)=1$, and

$$
\left\langle\Omega^{n} \otimes 1_{c}, D\left(\Omega^{n} \otimes \eta_{a}\right)\right\rangle,
$$

when $\mu\left(O_{a}\right)-\mu\left(O_{c}\right)=2$. In the case of the critical orbit $\theta$, we obtain the boundary component

$$
\left\langle\Omega^{n} \otimes 1_{c}, D\left(\Omega^{n} \otimes \theta\right)\right\rangle
$$

when $\mu(\theta)-\mu\left(O_{c}\right)=2$, where we consider the one-dimensional space $\hat{\mathcal{M}}\left(\theta, O_{c}\right)$ fibering over $O_{c}$ with zero-dimensional fiber. We also have the component

$$
\left\langle\Omega^{n} \otimes \theta, D\left(\Omega^{n} \otimes \eta_{a}\right)\right\rangle,
$$

when $\mu\left(O_{a}\right)-\mu(\theta)=1$, where the space $\hat{\mathcal{M}}\left(O_{a}, \theta\right)$ is one-dimensional. In this case the coefficient is obtained by integrating the 1-form $\eta_{a}$ over the 1-dimensional fiber of the endpoint map $e_{\theta}: \hat{\mathcal{M}}\left(O_{a}, \theta\right) \rightarrow \theta$.

Proof. For a given orbit $O_{a}$ the complex of equivariant forms is given by

$$
\Omega_{*, U(1)}\left(O_{a}\right)
$$

with the total grading

$$
\Omega_{j, U(1)}\left(O_{a}\right)=\bigoplus_{2 k+l=j} \mathbb{R} \Omega^{k} \otimes \Omega_{0}^{1-l}\left(O_{a}\right) .
$$

Here $\Omega$ is of degree 2 and $l$ is the grading in the dual de Rham complex where forms are graded by $\operatorname{dim}\left(O_{a}\right)-*$.

Thus we have

$$
\begin{aligned}
& \Omega_{0, U(1)}\left(O_{a}\right)=\mathbb{R}\left\langle 1 \otimes \eta_{a}\right\rangle, \\
& \Omega_{1, U(1)}\left(O_{a}\right)=\mathbb{R}\left\langle 1 \otimes 1_{a}\right\rangle, \\
& \Omega_{2, U(1)}\left(O_{a}\right)=\mathbb{R}\left\langle\Omega \otimes \eta_{a}\right\rangle,
\end{aligned}
$$




$$
\Omega_{3, U(1)}\left(O_{a}\right)=\mathbb{R}\left\langle\Omega \otimes 1_{a}\right\rangle,
$$

and so on, where $\eta_{a}$ is the one form that generates $H^{1}\left(S^{1}\right)$ and $1_{a}$ is the constant function equal to 1 on $O_{a}$.

The differential is given by $\partial_{G}=d-\tilde{\Omega} c(T)$, where $\tilde{\Omega}$ is the element in the dual of the Lie algebra $i \mathbb{R}$ such that $\langle\tilde{\Omega}, \Omega\rangle=1$ under the trace pairing, and $c(T)$ is the contraction with the vector field $T$ generated by the infinitesimal action of $U(1)$.

As we have already discussed at the beginning of Section 5 , the differential $\partial_{U(1)}$ of the equivariant complex $\Omega_{*, U(1)}\left(O_{a}\right)$ acts as

$$
D\left(\Omega^{n} \otimes 1_{a}\right)=0
$$

and

$$
D\left(\Omega^{n} \otimes \eta_{a}\right)=-\Omega^{n-1} 1 \otimes 1_{a} .
$$

The homology of this complex (for a fixed irreducible orbit $O_{a}$ ) is therefore one copy of $\mathbb{R}$ (corresponding to the generator $1 \otimes \eta_{a}$ ) in degree zero and zero in all other degrees. This is just the usual result that the equivariant homology of $O_{a}$ with a free action of $U(1)$ is isomorphic to the ordinary homology (with real coefficients) of the quotient, that is of a point.

Now let us consider the bigraded complex where several orbits are considered. The bigraded complex is of the form

$$
\bigoplus_{\mu\left(O_{a}\right)+j=*} \Omega_{j, U(1)}\left(O_{a}\right)
$$

The equivariant boundary operator can be written explicitly in components of the form

$$
\begin{array}{ccc}
\Omega^{n} \otimes 1_{a} & \mapsto & -n_{a b} \Omega^{n} \otimes 1_{b} \\
D: & & \mapsto \\
\Omega^{n} \otimes \eta_{a} & \left.\mapsto n_{a b} \Omega^{n} \otimes \eta_{b}\right) \oplus\left(m_{a c} \Omega^{n} \otimes 1_{c}\right) \\
& & \oplus\left(-\Omega^{n-1} \otimes 1_{a}\right) .
\end{array}
$$

In the case of the generator $\theta$, there are extra components as in the statement of the Proposition. Here $n_{a b}=\# \hat{\mathcal{M}}\left(O_{a}, O_{b}\right)^{*}$, where the relative index is $\mu\left(O_{a}\right)-\mu\left(O_{b}\right)=1$, so that $-\left(e_{b}^{-}\right)_{*}\left(e_{a}^{+}\right)^{*} 1_{a}=-n_{a b} 1_{b}$ and $\left(e_{b}^{-}\right)_{*}\left(e_{a}^{+}\right)^{*} \eta_{a}=n_{a b} \eta_{b}$. The coefficient $m_{a c}$ comes from the integration of the one-form $\eta_{a}$ over the one-dimensional fiber of the moduli space $\mathcal{M}\left(O_{a}, O_{c}\right)^{*}$ with $\mu\left(O_{a}\right)-\mu\left(O_{c}\right)=2$, namely $\left(e_{c}^{-}\right)_{*}\left(e_{a}^{+}\right)^{*} \eta_{a}=m_{a c} 1_{c}$. The components of $D$ are represented in the following diagram. 


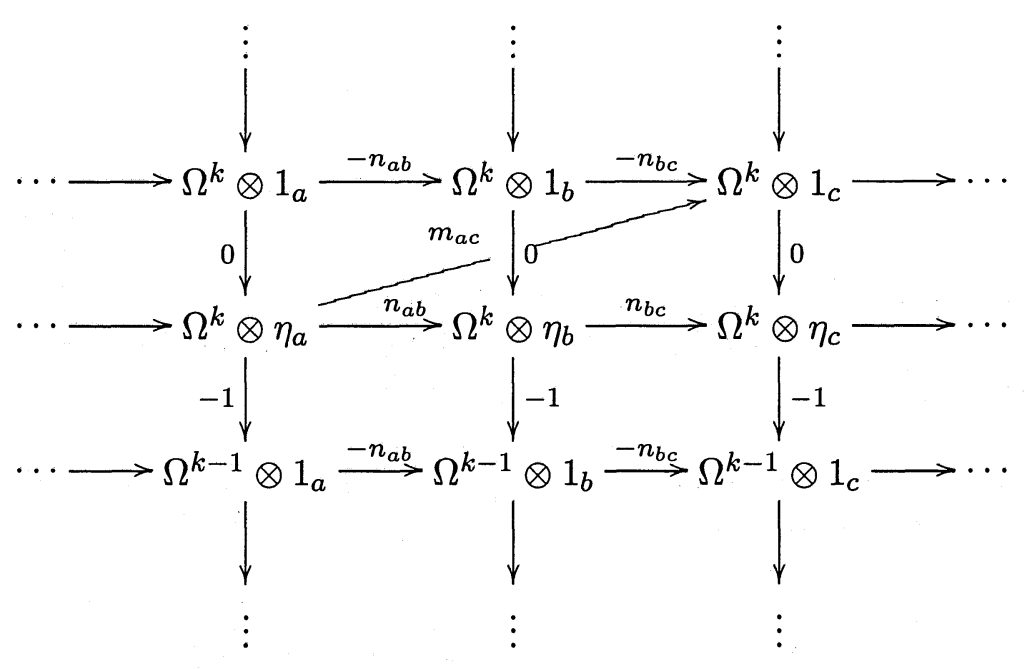

Remark 5.4. In view of the result of Proposition 5.3, we notice that the full strength of Theorem 4.23 is in fact more than what is strictly necessary in order to prove that the boundary $D$ of the equivariant Floer complex satisfies $D^{2}=0$. In fact, knowing that the boundary operator only depends on the moduli spaces $\hat{\mathcal{M}}\left(O_{a}, O_{b}\right)$ of dimension at most two, makes it only necessary, strictly speaking, to know the properties of the compactification for moduli spaces $\hat{\mathcal{M}}\left(O_{b}, O_{c}\right)$ of dimension at most three.

Clearly, it does not make any substantial difference to derive the properties of the fine structure of the compactification $\hat{\mathcal{M}}\left(O_{a}, O_{b}\right)^{*}$ in this reduced case or in the general case proved in Theorem 4.23. However, both here and in Section 6, where we analyze the case of obstructed gluing, it seems useful to identify explicitly what is the minimum requirement on the fine structure of the compactification that is needed in the arguments, cf. Remark 6.2 and Remark 6.3.

\subsection{Intermezzo: the coefficient $m_{a c}$.}

We now give a topological description of the coefficient $m_{a c}$ that appears in the component

$$
\left\langle D\left(\Omega^{n} \otimes \eta_{a}\right), \Omega^{n} \otimes 1_{c}\right\rangle=m_{a c}
$$

of the boundary operator, for $\mu\left(O_{a}\right)-\mu\left(O_{c}\right)=2$. This coefficient is defined as the pullback and pushforward of the 1 -form $\eta_{a}$ along the $U(1)$-equivariant 
endpoint fibrations

$$
e_{a}^{+}: \hat{\mathcal{M}}\left(O_{a}, O_{c}\right)^{*} \rightarrow O_{a}
$$

and

$$
e_{c}^{-}: \hat{\mathcal{M}}\left(O_{a}, O_{c}\right)^{*} \rightarrow O_{c}
$$

that is,

$$
m_{a c}=\left(e_{c}^{-}\right)_{*}\left(e_{a}^{+}\right)^{*} \eta_{a} .
$$

The 2-dimensional $U(1)$-manifold $\hat{\mathcal{M}}\left(O_{a}, O_{c}\right)^{*}$ fibers over the circles $O_{a}$ and $O_{c}$ with 1-dimensional fibers. Topologically, $\hat{\mathcal{M}}\left(O_{a}, O_{c}\right)^{*}$ consists of a collection of finitely many cylinders and finitely many tori.

Lemma 5.5. The pullback 1-form $\left(e_{a}^{+}\right)^{*} \eta_{a}$ defines a Cartan connection on the principal $U(1)$-bundle $\hat{\mathcal{M}}\left(O_{a}, O_{c}\right) \rightarrow \hat{\mathcal{M}}(a, c)$.

Proof. We want to obtain a splitting of the exact sequence

$$
0 \rightarrow \mathcal{T}(U(1)) \rightarrow \mathcal{T} \hat{\mathcal{M}}\left(O_{a}, O_{c}\right) \rightarrow \mathcal{T} \hat{\mathcal{M}}\left(O_{a}, O_{c}\right) / \mathcal{T}(U(1)) \cong \mathcal{T} \hat{\mathcal{M}}(a, c) \rightarrow 0
$$

Since the endpoint map $e_{a}^{+}$is $U(1)$-equivariant, and $\eta_{a}$ is the generator of $H^{1}\left(O_{a}\right)$, the kernel $\operatorname{Ker}\left(\left(e_{a}^{+}\right)^{*} \eta_{a}\right)$ defines a horizontal subspace of $\mathcal{T} \hat{\mathcal{M}}\left(O_{a}, O_{c}\right)$.

Lemma 5.6. Over each component of $\hat{\mathcal{M}}\left(O_{a}, O_{c}\right)$, the pushforward

$$
\left(e_{c}^{-}\right)_{*}\left(e_{a}^{+}\right)^{*} \eta_{a}
$$

computes the winding number $W(\gamma)$ of the 1-dimensional $\gamma=\left(e_{c}^{-}\right)^{-1}\left(x_{c}\right)$, for a point $x_{c} \in O_{c}$, around the fiber of the $U(1)$-fibration $\pi: \hat{\mathcal{M}}\left(O_{a}, O_{c}\right) \rightarrow$ $\hat{\mathcal{M}}(a, c)$.

Proof. Consider a connected component $\hat{X}$ of $\hat{\mathcal{M}}(a, c)$. For $\mu\left(O_{a}\right)-\mu\left(O_{c}\right)=$ 2 , the component

$$
\pi^{-1}(\hat{X}) \subset \hat{\mathcal{M}}\left(O_{a}, O_{c}\right)
$$

is a cylinder or a torus. Choosing a point $x_{c} \in O_{c}$, one can identify the fiber

$$
\left(e_{c}^{-}\right)^{-1}\left(x_{c}\right) \cap \pi^{-1}(\hat{X})
$$

with a lift $\gamma$ of the path $\hat{X} \subset \hat{\mathcal{M}}(a, c)$ to $\hat{\mathcal{M}}\left(O_{a}, O_{c}\right)$. In fact, the endpoint fibration $e_{c}^{-}$is compatible with the boundary strata $\hat{\mathcal{M}}\left(O_{a}, O_{b}\right) \times_{O_{b}}$ 
$\hat{\mathcal{M}}\left(O_{b}, O_{c}\right)$. Since the 1-form $\eta_{a}$ is the generator of $H^{1}\left(O_{a}\right)$, the pullback $\left(e_{a}^{+}\right)^{*} \eta_{a}$ integrated along the path

$$
\gamma=\left(e_{c}^{-}\right)^{-1}\left(x_{c}\right) \cap \pi^{-1}(\hat{X})
$$

measures precisely the number of times this path winds around the fibers of the map $\pi$,

$$
\int_{\left(e_{c}^{-}\right)^{-1}\left(x_{c}\right) \cap \pi^{-1}(\hat{X})}\left(e_{a}^{+}\right)^{*} \eta_{a}=W(\gamma) .
$$

Notice that in $\hat{\mathcal{M}}\left(O_{a}, O_{c}\right)$ we have a choice of a horizontal direction $\ell\left(x_{a}\right)$, given by the integral lines of $\operatorname{Ker}\left(\left(e_{a}^{+}\right)^{*} \eta_{a}\right)$, for any initial condition $x_{a} \in O_{a}$, and a direction given by the path $\left(e_{c}^{-}\right)^{-1}\left(x_{c}\right)$ for fixed $x_{c}$ in $O_{c}$. The horizontal line $\ell\left(x_{a}\right)$ can be identified with the fiber of the other endpoint map $\left(e_{a}^{+}\right)^{-1}\left(x_{a}\right)$. Both the lines $\left(e_{c}^{-}\right)^{-1}\left(x_{c}\right)$ and $\left(e_{a}^{+}\right)^{-1}\left(x_{a}\right)$ represent a homeomorphic lift of $\hat{\mathcal{M}}(a, c)$ to $\hat{\mathcal{M}}\left(O_{a}, O_{c}\right)$, however, in general, an element $[\mathbb{A}, \Psi] \in\left(e_{c}^{-}\right)^{-1}\left(x_{c}\right)$ has a limit at $t \rightarrow-\infty$ which is some $\lambda x_{a}$ in $O_{a}$, not equal to $x_{a}$, and similarly an element $[\mathbb{A}, \Psi] \in\left(e_{a}^{+}\right)^{-1}\left(x_{a}\right)$ has a limit at $t \rightarrow+\infty$ which is some $\lambda x_{c}$ in $O_{c}$ not equal to $x_{c}$. Different elements in $\left(e_{a}^{+}\right)^{-1}\left(x_{a}\right)$ have different $\lambda x_{c}$ limits in $O_{c}$ and similarly for the other endpoint fibration. The intersection between the two lines then consists of finitely many points. By our identification of $\ell\left(x_{a}\right)=\left(e_{a}^{+}\right)^{-1}\left(x_{a}\right)$ with the horizontal direction, this number is equal to the winding number of the path $\left(e_{c}^{-}\right)^{-1}\left(x_{c}\right)$. This intersection number counts the solutions $[\mathbb{A}, \Psi] \in \hat{\mathcal{M}}\left(O_{a}, O_{c}\right)$ that actually have prescribed endpoints $x_{a}$ and $x_{c}$ at both ends.

Now consider the $U(1)$-bundle $\mathcal{M}\left(O_{a}, O_{c}\right) \rightarrow \mathcal{M}(a, c)$ over the parameterized moduli space. Connected components $X$ of $\mathcal{M}(a, c)$ are infinite cylinders or strips of the form $X \cong[0,1] \times \mathbb{R}$. Consider a choice of a base point $\left(y_{0}, t_{0}\right)$ on $Y \times \mathbb{R}$, and a complex line $\ell_{y_{0}}$ in the fiber $W_{y_{0}}$ of the spinor bundle $W=S \otimes L$ over $y_{0} \in Y$. We choose $\ell_{y_{0}}$ so that it does not contain the spinor part $\psi$ of any irreducible critical point. Since there are only finitely many critical points we can guarantee such choice exists. We consider the line bundle

$$
\mathcal{L}_{a c}=\mathcal{M}\left(O_{a}, O_{c}\right) \times_{U(1)}\left(W_{y_{0}} / \ell_{y_{0}}\right) \rightarrow \mathcal{M}(a, c)
$$

with a section given by

$$
s([\mathbb{A}, \Psi])=\left([\mathbb{A}, \Psi], \Psi\left(y_{0}, t_{0}\right)\right) .
$$


Lemma 5.7. For a generic choice of $\left(y_{0}, t_{0}\right)$ the section $s$ of (74) has no zeroes on the boundary strata $\hat{\mathcal{M}}(a, b) \times \hat{\mathcal{M}}(b, c)$. This determines a trivialization of $\mathcal{L}_{a c}$ away from a compact set in $\mathcal{M}(a, c)$. The line bundle $\mathcal{L}_{a c}$ over $\mathcal{M}(a, c)$, with the trivialization $\varphi$ specified above, has relative Euler class satisfying

$$
e\left(\mathcal{L}_{a c}, \varphi\right)=m_{a c}
$$

Proof. The section $s$ induces a trivialization of the line bundle $\mathcal{L}_{a c}$ away from a compact set in $\mathcal{M}(a, c)$. In fact, for $T \rightarrow \infty$, we have

$$
s\left((\mathbb{A}, \Psi)^{-T}\right) \rightarrow s\left(\left(A_{a}, \psi_{a}\right)\right) \neq 0
$$

and

$$
s\left((\mathbb{A}, \Psi)^{T}\right) \rightarrow s\left(\left(A_{c}, \psi_{c}\right)\right) \neq 0 .
$$

This corresponds to trivializing the fibration $\hat{\mathcal{M}}\left(O_{a}, O_{c}\right) \times\{T\}$ with the horizontal lines $\ell\left(x_{a}, \lambda\right)=\left(e_{a}^{+}\right)^{-1}\left(\lambda x_{a}\right)$, for $T \leq-T_{0}$ and with the lines $\ell\left(x_{c}, \lambda\right)=\left(e_{c}^{-}\right)^{-1}\left(\lambda x_{c}\right)$ for $T \geq T_{0}$. The obstruction to extending the trivialization over all of $\mathcal{M}(a, c)$ is then measured precisely by the winding number $m_{a c}$. This is therefore the relative Euler class of $\mathcal{L}_{a c}$, which can also be computed as

$$
e\left(\mathcal{L}_{a c}, \varphi\right)=\# s^{-1}(0) .
$$

In particular, this implies that the coefficient $m_{a c}$ can be computed by counting zeroes of any transverse section

$$
s: \mathcal{M}(a, c) \rightarrow \mathcal{L}_{a c}
$$

which is non-vanishing away from a compact set, and induces the same trivialization $\varphi$,

$$
m_{a c}=\# s^{-1}(0) .
$$

We discuss some identities satisfied by the coefficients $m_{a c}$.

Remark 5.8. Let $a$ and $d$ be two irreducible critical points with $\mu(a)-$ $\mu(d)=3$. Assume that all the critical points $c_{i}$ with $\mu(a)>\mu\left(c_{i}\right)>\mu(d)$ are irreducible. Then we have the identity

$$
\sum_{c_{1}: \mu(a)-\mu\left(c_{1}\right)=1} n_{a, c_{1}} m_{c_{1}, d}-\sum_{c_{2}: \mu\left(c_{2}\right)-\mu(d)=1} m_{a, c_{2}} n_{c_{2}, d}=0 .
$$


When the reducible critical point $\theta$ has index between $(\mu(a), \mu(c))$, with $\mu(a)=1$ and $\mu(d)=-2$, we have the identity

$$
\sum_{c_{1}: \mu\left(c_{1}\right)=0} n_{a, c_{1}} m_{c_{1}, d}+n_{a \theta} n_{\theta d}-\sum_{c_{2}: \mu\left(c_{2}\right)=-1} m_{a, c_{2}} n_{c_{2}, d}=0 .
$$

This identity follows directly from the definition of the invariant $m_{a c}$ as pullback and pushforward of the 1-form $\eta_{a}$, using Stokes' theorem for fibrations with boundary on the compactified moduli space $\hat{\mathcal{M}}\left(O_{a}, O_{d}\right)^{*}$. The sign comes from the orientations in the gluing theorem. However, the identity (75) also has a topological interpretation in terms of relative Euler classes. In fact, the counting above is the counting of the boundary points of the zero set of a strata transverse section of the line bundle $\mathcal{L}_{a d}$ over the unparameterized moduli space $\mathcal{M}(a, d)$. For such section, $s^{-1}(0)$ is a 1-dimensional manifold with boundary. The counting above corresponds to a section that has

$$
\begin{gathered}
\partial s^{-1}(0)=\bigcup_{c_{1}: \mu(a)-\mu\left(c_{1}\right)=1} \hat{\mathcal{M}}\left(a, c_{1}\right) \times\left(s^{-1}(0) \cap \mathcal{M}\left(c_{1}, d\right)\right) \\
\cup \bigcup_{c_{2}: \mu\left(c_{2}\right)-\mu(d)=1}\left(-s^{-1}(0) \cap \mathcal{M}\left(a, c_{2}\right)\right) \times \hat{\mathcal{M}}\left(c_{2}, d\right) .
\end{gathered}
$$

The other identity can be proved by the similar methods, with an extra $U(1)$ gluing parameter when gluing along $\theta$, as in Lemma 2.16 .

Now consider the case of the 2-dimensional moduli spaces $\hat{\mathcal{M}}\left(O_{a}, \theta\right)$, for $\mu\left(O_{a}\right)-\mu(\theta)=2$, and $\hat{\mathcal{M}}\left(\theta, O_{c}\right)$ for $\mu(\theta)-\mu\left(O_{c}\right)=3$, with $\theta$ the unique reducible (the $U(1)$-fixed point in $\mathcal{M}^{0}$ ).

The moduli spaces $\hat{\mathcal{M}}\left(O_{a}, \theta\right)$, with $\mu\left(O_{a}\right)-\mu(\theta)=2$, do not contribute components to the boundary operator $D$ of the Floer complex, because we have

$$
\left(e_{\theta}\right)_{*}\left(e_{a}^{+}\right)^{*} \eta_{a}=0,
$$

since the fibers of the endpoint map

$$
e_{\theta}: \hat{\mathcal{M}}\left(O_{a}, \theta\right) \rightarrow \theta
$$

are 2-dimensional.

It is still true that $\left(e_{a}^{+}\right)^{*} \eta_{a}$ defines a connection on the principal $U(1)$ bundle over $\mathcal{M}(a, \theta)$. Moreover, a component $\hat{X} \subset \hat{\mathcal{M}}(a, \theta)$ is again topologically a circle or a line segment. Given a choice of a lift $\gamma$ of $\hat{X}$ inside the 
component $\pi^{-1}(\hat{X}) \subset \hat{\mathcal{M}}\left(O_{a}, \theta\right)$, we can still compute its winding number and follow the same construction given above. However, now the choice of the lift $\gamma$ is no longer determined by the endpoint fibration

$$
e_{\theta}: \hat{\mathcal{M}}\left(O_{a}, \theta\right) \rightarrow \theta .
$$

Different possible choices of the lift $\gamma$ can have different winding numbers $W(\gamma)$.

Similarly, we can still construct the bundle $\mathcal{L}_{a, \theta}$ over $\mathcal{M}(a, \theta)$. However, the Section (74), in this case, does not give a trivialization away from a compact set. Similarly, the fibers of the endpoint map $e_{\theta}$ do not provide a trivialization at the $T \rightarrow \infty$ end, since they do not determine a lift of $\mathcal{M}(a, \theta)$.

However, we claim that it is still possible to associate an invariant $m_{a \theta}$ to the moduli space $\mathcal{M}(a, \theta)$, which can be interpreted topologically as a relative Euler class. We briefly illustrate here why this should be the case, then in Section 6.3, in Remark 6.24, we give a definition of the invariant $m_{a \theta}$ as counting of the zeroes of a particular section of $\mathcal{L}_{a, \theta}$, the obstruction section.

The choice of the base point $\left(y_{0}, t_{0}\right)$ determines a trivialization of the $U(1)$-fibration $\mathcal{B}^{0}$ over the configuration space $\mathcal{B}$, in a small neighborhood of each irreducible critical point. If we think of parameterized flow lines between two critical points $a$ and $c$ as embedded in the irreducible part $\mathcal{B}^{*}$ of $\mathcal{B}$, then the $U(1)$-fibration $\mathcal{M}\left(O_{a}, O_{c}\right)$ has an induced trivialization away from a compact set in $\mathcal{M}(a, c)$, which agrees with the trivialization $\varphi$ given by the cross section (74) of the associated line bundle $\mathcal{L}_{a c}$. We can choose a small neighborhood $U_{\theta}$ of the reducible critical point $\theta$ such that $U_{\theta}^{*}=U_{\theta} \cap \mathcal{B}^{*}$ is 1-connected, hence there is also a unique trivialization of $\mathcal{B}^{0}$ over $U_{\theta}^{*}$. We assume that this trivialization is given by a constant cross section. Thus, there is an induced trivialization away from a compact set in $\mathcal{M}(a, \theta)$ (where $\mu\left(O_{a}\right)-\mu(\theta)=2$ ) or $\mathcal{M}\left(\theta, O_{c}\right)$ (where $\mu(\theta)-\mu\left(O_{c}\right)=3$ ). Using this trivialization, we can define $m_{a \theta}$ and $m_{\theta c}$ to be the relative Euler class of the corresponding associated line bundles $\mathcal{L}_{a \theta}$ and $\mathcal{L}_{\theta c}$, respectively.

We return to the description of the invariant $m_{a c}$ for the case of $O_{a}$ and $O_{c}$ free orbits of relative index two, in order to illustrate the point of view we shall adopt in Remark 6.24 in describing the invariant $m_{a \theta}$.

In general, we can construct the relative Euler class which is a cycle defined by the zeros of the transverse section as follows. Over the unparameterized moduli space $\hat{\mathcal{M}}(a, c)$, consider the line bundle

$$
\mathcal{L}_{a c}=\hat{\mathcal{M}}\left(O_{a}, O_{c}\right) \times_{U(1)} \mathbb{C},
$$


associated to the principal $U(1)$-fibration

$$
\pi: \hat{\mathcal{M}}\left(O_{a}, O_{c}\right) \rightarrow \hat{\mathcal{M}}(a, c) .
$$

The pullback under the quotient map $\mathcal{M}(a, c) \rightarrow \hat{\mathcal{M}}(a, c)$ induces a pullback line bundle, which we still denote $\mathcal{L}_{a c}$, over the parameterized moduli space $\mathcal{M}(a, c)$.

Lemma 5.9. Over the boundary strata

$$
\hat{\mathcal{M}}(a, b) \times \hat{\mathcal{M}}(b, c)
$$

of $\hat{\mathcal{M}}(a, c)$, the line bundle $\mathcal{L}_{a c}$ has the form

$$
\mathcal{L}_{a c} \cong \pi_{1}^{*} \mathcal{L}_{a b} \otimes \pi_{2}^{*} \mathcal{L}_{b c}
$$

Proof. The result follows by showing that, for all critical orbits $O_{p}$ and $O_{q}$ with $\mu\left(O_{p}\right)>\mu\left(O_{q}\right)$, the principal $U(1)$-bundles

$$
\pi: \hat{\mathcal{M}}\left(O_{p}, O_{q}\right) \rightarrow \hat{\mathcal{M}}(p, q)
$$

are compatible with the gluing maps, namely they define a line bundle

$$
\mathcal{L}_{p q}=\hat{\mathcal{M}}\left(O_{p}, O_{q}\right)^{*} \times_{U(1)} \mathbb{C} \rightarrow \hat{\mathcal{M}}(p, q)^{*}
$$

in the category of manifolds with corners. The compatibility of the $U(1)$ fibration with the gluing maps follows from our proof of Theorem 4.9.

We now specify a choice of a class of transverse sections of $\mathcal{L}_{a c}$ over $\mathcal{M}(a, c)$. We prescribe a choice of non-zero complex numbers $s_{a}$ for each critical point $a$. For each pair of critical points $a, b$ of relative index 1 , we choose a path of non-zero complex numbers $s_{a b}: \mathbb{R} \rightarrow \mathbb{C}-\{0\}$ connecting $s_{a}$ and $s_{b}$, which is contractible in $\mathbb{C}-\{0\}$ and is constant outside a compact set in $\mathbb{R}$. Over the 1-dimensional $\mathcal{M}(a, b)$ we define nowhere vanishing sections of $\mathcal{L}_{a b}$ as pullbacks of the $s_{a b}$ on $\hat{\mathcal{M}}(a, b)$. We still denote these sections over $\mathcal{M}(a, b)$ by $s_{a b}$. Over the 2-dimensional $\mathcal{M}(a, c)$ we consider the class of all transverse sections $s_{a c}$ of $\mathcal{L}_{a c}$ that satisfy

$$
s_{a c}=\pi_{1}^{*} s_{a b} \otimes \pi_{2}^{*} s_{b c}
$$

over the product submanifolds

$$
\hat{\mathcal{M}}(a, b) \times \hat{\mathcal{M}}(b, c) \times[0, \epsilon) \times \mathbb{R}
$$


where $[0, \epsilon)$ is the gluing parameter, and such that, for a sufficiently large $T, s_{a c}$ is the constant section $s_{a}$ over

$$
\hat{\mathcal{M}}(a, c) \times(-\infty,-T]
$$

and the constant section $s_{c}$ over

$$
\hat{\mathcal{M}}(a, c) \times[T, \infty) .
$$

Clearly, any such section $s_{a c}$ gives the same trivialization $\varphi$ of $\mathcal{L}_{a c}$ away from a compact set in $\mathcal{M}(a, c)$. We can therefore compute the relative Euler class by counting the zeros of this transverse section, that is,

$$
e\left(\mathcal{L}_{a c}, \varphi\right)=\# s_{a c}^{-1}(0) .
$$

For higher dimensional moduli spaces $\mathcal{M}(a, c)$, we proceed analogously, by inductively defining the class of transverse sections of $\mathcal{L}_{a c}$ which are compatible with the boundary strata of $\mathcal{M}(a, c)$, that is, we require that

$$
s_{a c}=\pi_{1}^{*} s_{a b} \otimes \pi_{2}^{*} s_{b c}
$$

over all the product submanifolds

$$
\hat{\mathcal{M}}(a, b) \times \hat{\mathcal{M}}(b, c) \times[0, \epsilon) \times \mathbb{R}
$$

and that $s_{a c}$ agrees with the constant section $s_{a}$ over

$$
\hat{\mathcal{M}}(a, c) \times(-\infty,-T]
$$

and with the constant section $s_{c}$ over

$$
\hat{\mathcal{M}}(a, c) \times[T, \infty) .
$$

Lemma 5.10. Consider the line bundle $\mathcal{L}_{a c}$ over a parameterized moduli space $\mathcal{M}(a, c)$, where $\mu(a)-\mu(c)=3$ and $a$ is irreducible or where $a$ is reducible and $\mu(c)=-4$, with the cross section $s_{a c}$ as above. Then the zero set $s_{a c}^{-1}(0)$ is a co-dimension 2 submanifold of $\mathcal{M}(a, c)$ which can be compactified by adding lower dimensional boundary strata such that the codimension one boundary strata are given by

$$
\partial s_{a c}^{-1}(0)=\bigcup_{\mu(b)-\mu(c)=1} s_{a b}^{-1}(0) \times \hat{\mathcal{M}}(b, c)
$$




$$
\cup \underset{\mu(b)-\mu(c)=2}{\bigcup} \hat{\mathcal{M}}(a, b) \times s_{b c}^{-1}(0)
$$

for $\mu(a) \neq 1$;

$$
\begin{gathered}
\partial s_{a c}^{-1}(0)=\bigcup_{\mu(b)-\mu(c)=1} s_{a b}^{-1}(0) \times \hat{\mathcal{M}}(b, c) \\
\cup \bigcup_{\mu(a)-\mu(b)=1} \hat{\mathcal{M}}(a, b) \times s_{b c}^{-1}(0) \\
\cup s_{a c}^{-1}(0) \cap(\hat{\mathcal{M}}(a, \theta) \times \mathcal{M}(\theta, c) \times U(1))
\end{gathered}
$$

for $\mu(a)=1$ and $\mu(c)=-2$.

Proof. The result follows from the choice of the class of sections $s_{a c}$, together with the result of Lemma 5.9.

If we compare the results of Lemma 5.9 and Lemma 5.10, with Lemma 5.7 , we see that our previous definitions of the invariant

$$
m_{a c}=e\left(\mathcal{L}_{a c}, \varphi\right)=\# s_{a c}^{-1}(0)
$$

are a particular case of the procedure illustrated here. In particular, the identities of Remark 5.8 are then the direct consequences of Lemma 5.10.

\section{Topological invariance.}

In the present section we show that the equivariant Floer groups, defined as in Definition 5.2, are topological invariants, in the sense that they are independent of the choice of the metric and of the perturbation.

Throughout all this discussion, recall that the Floer groups depend on

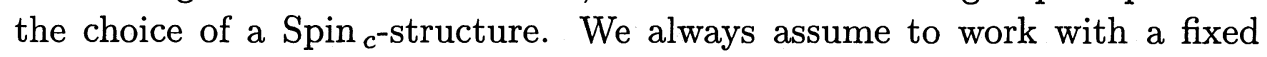
Spin $_{c}$-structure, so we never mention explicitly this dependence.

The easiest case, for the proof of topological invariance, is under the assumption that $b^{1}(Y) \geq 2$. In fact, in this case there are no reducible solutions, hence a cobordism argument can be used to construct a morphism of the chain complexes that gives an isomorphism of the cohomology groups. In the case when $b^{1}(Y)=1$, the metric and the perturbation cannot be chosen arbitrarily. This was already noticed in [5] in the case of the invariant associated to the moduli space $\mathcal{M}$ (which is the Euler characteristic of the Floer homology, [38]). However, given two generic metrics, it is possible 
to find a path of metrics joining them and an open set of sufficiently small perturbations $\rho$ such that the invariant does not change. The dependence which is introduced in this case is rather mild and it amounts to a choice of a homology class in $H^{1}(Y ; \mathbb{Z})$. Thus, the $b^{1}(Y) \geq 1$ case will follow by exactly the same argument that we present below in our simple model case of same chamber metrics and perturbations.

The case of a homology sphere [13] is rather different. In fact in this case there is an explicit dependence on the metric ([15], [31]). The interesting fact about the equivariant Floer homology is that it is instead metric independent. This means that, in this case, though we still have a condition on the kernel of the Dirac operator $\left(\operatorname{Ker}\left(\partial_{A}\right)\right.$ trivial at the reducible solution) that breaks the space of metrics and perturbations into chambers with co-dimension one walls, it is however possible to construct a chain homomorphism between the Floer complexes that correspond to different metrics, and a chain homotopy. That is, for the equivariant Floer homology the wall crossing consists only of a global grade shift.

The main theorem of this section is the following.

Theorem 6.1. Let $Y$ be a rational homology sphere with fixed $\operatorname{Spin}_{c^{-}}$ structure. Suppose given two metrics $g_{0}$ and $g_{1}$ on $Y$ and perturbations $\nu_{0}$ and $\nu_{1}$. Then there exist a chain homomorphism I between the equivariant Floer complexes $\left(C_{* U(1)\left(g_{0}, \nu_{0}\right)}, D\right)$ and $\left(C_{* U(1)\left(g_{1}, \nu_{1}\right)}, D\right)$, defined by considering a generic path $\left(g_{t}, \nu_{t}\right)$ and moduli spaces $\mathcal{M}\left(O_{a}, O_{a^{\prime}}\right)$ of solutions of the flow equations on $\left(Y \times \mathbb{R}, g_{t}+d t^{2}\right)$. This chain homomorphism induces an isomorphism in homology up to an index shift given by the spectral flow of the Dirac operator $\partial_{\nu_{t}}^{g_{t}}$. The analogous construction works for cohomology groups.

We first give a "model proof" in the much simpler case of two metrics and perturbations $\left(g_{0}, \nu_{0}\right)$ and $\left(g_{1}, \nu_{1}\right)$ that are in the same chamber. The proof in this case is obviously much simpler than the general statement of Theorem 6.1. In fact, the interesting part of the statement is only for different chambers. The reason why we present explicitly the proof of this case is because, once we know that all the $H F_{*, U(1)}^{S W}(Y,(g, \nu))$, for $(g, \nu)$ in the same chamber, are isomorphic, we can prove the general case just by analyzing the case of two sufficiently close metrics and perturbations $\left(g_{0}, \nu_{0}\right)$ and $\left(g_{1}, \nu_{1}\right)$ in two different chambers, across a codimension one wall. Moreover, having the model case presented explicitly, we can discuss the case of different chambers by pointing out the various points in the proof where the original argument has to be modified. We prove the general 
statement of Theorem 6.1 in Section 6.3.

Proof, Part I: the same chamber case.

Choose a path of metrics and perturbations $\left(g_{t}, \nu_{t}\right)$ with $t \in[0,1]$ that interpolates between $\left(g_{0}, \nu_{0}\right)$ and $\left(g_{1}, \nu_{1}\right)$. Consider the manifold $Y \times \mathbb{R}$ endowed with the metric $g_{t}+d t^{2}$ on $Y \times\{t\}$ extended as the product metric outside $Y \times[0,1]$. Consider on $Y \times \mathbb{R}$ the perturbed "gradient flow" equations with respect to the metric $g_{t}$. Denote $\left\{O_{a}, O_{b}, \cdots\right\}$ the critical orbits for the metric $g_{0}$ and $\left\{O_{a^{\prime}}, O_{b^{\prime}}, \cdots\right\}$ the critical orbits for the metric $g_{1}$.

We adopt the convention of fixing the absolute grading of the Floer complex to be the relative grading with respect to the reducible solution $\mu\left(O_{a}\right)-\mu\left(\theta_{0}\right)$, and $\mu\left(O_{a^{\prime}}\right)-\mu\left(\theta_{1}\right)$, where $\theta_{0}=\left[\nu_{0}, 0\right]$ and $\theta_{1}=\left[\nu_{1}, 0\right]$. We perform a shift of grading in the complex $C_{k U(1)}\left(Y, g_{1}, \nu_{1}\right)$ by setting $\mu\left(\theta_{1}\right)=-S F\left(\partial_{\nu_{t}}^{g_{t}}\right)$, where $S F\left(\partial_{\nu_{t}}^{g_{t}}\right)$ is the spectral flow of the Dirac operator along the path of reducible solutions $\left[\nu_{t}, 0\right]$. In the same chamber case this shift is irrelevant, since the spectral flow is trivial, but it will be relevant in the general case we prove in Section 6.3.

We have moduli spaces $\mathcal{M}\left(O_{a}, O_{a^{\prime}}\right)$ of solutions modulo gauge transformations of suitably perturbed Seiberg-Witten equations on the manifold $\left(Y \times \mathbb{R}, g_{t}+d t^{2}\right)$, as specified in (87) and (88).

We need an analogue of Proposition 2.14 which ensures that, under a generic choice of the perturbation, the moduli spaces $\mathcal{M}\left(O_{a}, O_{a^{\prime}}\right)$ are smooth manifolds of dimension $\mu\left(O_{a}\right)-\mu\left(O_{a^{\prime}}\right)+\operatorname{dim}\left(O_{a}\right)$, where $\mu\left(O_{a^{\prime}}\right)$ denotes the shifted grading. In Lemma 6.5, Lemma 6.6, Corollary 6.7, and Lemma 6.8 we deal with these transversality issues.

We construct a degree zero homomorphism of the Floer complexes

$$
I: C_{k U(1)}\left(Y, g_{0}, \nu_{0}\right) \rightarrow C_{k U(1)}\left(Y, g_{1}, \nu_{1}\right) .
$$

We define the homomorphism $I$ as

$$
I_{a, a^{\prime}} \eta= \begin{cases}\left(e_{a^{\prime}}^{-}\right)_{*}\left(e_{a}^{+}\right)^{*} \eta & \mu\left(O_{a}\right)-\mu\left(O_{a^{\prime}}\right) \geq 0 \\ 0 & \mu\left(O_{a}\right)-\mu\left(O_{a^{\prime}}\right)<0\end{cases}
$$

with $\eta \in \Omega_{k-\mu\left(O_{a}\right), U(1)}\left(O_{a}\right)$. The maps $e_{a^{\prime}}^{-}$and $e_{a}^{+}$are the end point maps for $\mathcal{M}\left(O_{a}, O_{a^{\prime}}\right)$. Clearly, such $I$ commutes with $\Omega c(T)$.

Requiring that the expression (76) is well defined, that is, that we have a well defined push-forward map $\left(e_{a^{\prime}}^{-}\right)_{*}$, implies checking some properties of the compactification of the moduli spaces $\mathcal{M}\left(O_{a}, O_{a^{\prime}}\right)$. For the same chamber case, the analysis of the boundary structure of $\mathcal{M}\left(O_{a}, O_{a^{\prime}}\right)$ follows closely the model developed in Section 4 for the compactification of the 
moduli spaces of flow lines. However, when adapting this argument to the general case of different chambers, this compactification will require a much more delicate analysis.

The next step is then to prove that the map $I$ is a chain homomorphism. We want the relation $I \circ D-D \circ I=0$ to be satisfied. This corresponds to the expression

$$
\begin{aligned}
& (I D-D I)(\eta)_{a, b^{\prime}} \\
& \left\{\begin{array}{l}
\left(e_{b^{\prime}}^{-}\right)_{*}\left(e_{a}^{+}\right)^{*} \partial_{U(1)} \eta-\partial_{U(1)}\left(e_{b^{\prime}}^{-}\right)_{*}\left(e_{a}^{+}\right)^{*} \eta \\
\text { for } O_{b}=O_{a} \text { and } \mu\left(O_{b}\right) \geq \mu\left(O_{b^{\prime}}\right) \\
\text { or } O_{a^{\prime}}=O_{b^{\prime}} \text { and } \mu\left(O_{a}\right) \geq \mu\left(O_{b^{\prime}}\right) \\
-(-1)^{r(\eta)} \sum_{\left\{b \mid \mu\left(O_{b}\right)<\mu\left(O_{a}\right)\right\}}\left(e_{b^{\prime}}^{-}\right)_{*}\left(e_{b}^{+}\right)^{*}\left(e_{b}^{-}\right)_{*}\left(e_{a}^{+}\right)^{*} \eta \\
+(-1)^{r\left(\left(e_{a^{\prime}}^{-}\right)_{*}\left(e_{a}^{+}\right)^{*} \eta\right)} \sum_{\left\{a^{\prime} \mid \mu\left(O_{a^{\prime}}\right)>\mu\left(O_{b^{\prime}}\right)\right\}}\left(e_{b^{\prime}}^{-}\right)_{*}\left(e_{a^{\prime}}^{+}\right)^{*}\left(e_{a^{\prime}}^{-}\right)_{*}\left(e_{a}^{+}\right)^{*} \eta, \\
\text { for } \mu\left(O_{a}\right)>\mu\left(O_{b}\right) \geq \mu\left(O_{b^{\prime}}\right) \text { and } \mu\left(O_{a}\right) \geq \mu\left(O_{a^{\prime}}\right)>\mu\left(O_{b^{\prime}}\right)
\end{array}\right.
\end{aligned}
$$

where the first line in the right hand side of (77) corresponds to $O_{b}=O_{a}$ and $\mu\left(O_{b}\right) \geq \mu\left(O_{b^{\prime}}\right)$ or $O_{a^{\prime}}=O_{b^{\prime}}$ and $\mu\left(O_{a}\right) \geq \mu\left(O_{b^{\prime}}\right)$, and the other terms correspond to $\mu\left(O_{a}\right)>\mu\left(O_{b}\right) \geq \mu\left(O_{b^{\prime}}\right)$ and $\mu\left(O_{a}\right) \geq \mu\left(O_{a^{\prime}}\right)>\mu\left(O_{b^{\prime}}\right)$.

In order to show that the right hand side is zero, we consider components of the form

$$
\begin{aligned}
& \bigcup_{\left\{a^{\prime} \mid \mu\left(O_{a^{\prime}}\right)>\mu\left(O_{b^{\prime}}\right)\right\}}\left(\mathcal{M}\left(O_{a}, O_{a^{\prime}}\right)^{*} \times{ }_{O_{a^{\prime}}} \hat{\mathcal{M}}\left(O_{a^{\prime}}, O_{b^{\prime}}\right)^{*}\right) \\
& \bigcup_{\left\{b \mid \mu\left(O_{b}\right)<\mu\left(O_{a}\right)\right\}}\left(-\hat{\mathcal{M}}\left(O_{a}, O_{b}\right)^{*} \times_{O_{b}} \mathcal{M}\left(O_{b}, O_{b^{\prime}}\right)^{*}\right),
\end{aligned}
$$

We want to show that these are precisely all the components of the ideal boundary that appear in the actual boundary of the compactification $\mathcal{M}\left(O_{a}, O_{b^{\prime}}\right)^{*}$. We need Stokes' theorem to apply, hence we need to prove that the compactification $\mathcal{M}\left(O_{a}, O_{a^{\prime}}\right)^{*}$ with boundary strata (78) has the structure of a smooth manifold with corners. We also need an analogue of Corollary 4.24, which shows that the endpoint maps

$$
e_{a}^{+}: \mathcal{M}\left(O_{a}, O_{a}^{\prime}\right)^{*} \rightarrow O_{a}
$$

and

$$
e_{a^{\prime}}^{-}: \mathcal{M}\left(O_{a}, O_{a}^{\prime}\right)^{*} \rightarrow O_{a^{\prime}}
$$

are fibrations compatible with the boundary strata and with compact fibers, as in Theorem 6.9. Thus, using Stokes' theorem again, we write the expression $\left(e_{a}^{+}\right)^{*} \eta$ in terms of the push-forward map on the co-dimension one 
boundary strata. This proves that (77) vanishes identically, as expected. Notice that the sign $(-1)^{r\left(\left(e_{a^{\prime}}^{-}\right)_{*}\left(e_{a}^{+}\right)^{*} \eta\right)}$ is just $(-1)^{r(\eta)}$.

Now consider another path of metrics and perturbations $\left(\tilde{g}_{t}, \tilde{\nu}_{t}\right)$ that connects $\left(g_{1}, \nu_{1}\right)$ to $\left(g_{0}, \nu_{0}\right)$. We construct the corresponding homomorphism

$$
J: C_{k U(1)}\left(Y, g_{1}, \nu_{1}\right) \rightarrow C_{k U(1)}\left(Y, g_{0}, \nu_{0}\right),
$$

as in the previous case,

$$
J_{a^{\prime}, a} \eta= \begin{cases}\left(e_{a}^{-}\right)_{*}\left(e_{a^{\prime}}^{+}\right)^{*} \eta & \mu\left(O_{a^{\prime}}\right)-\mu\left(O_{a}\right) \geq 0 \\ 0 & \mu\left(O_{a^{\prime}}\right)-\mu\left(O_{a}\right)<0\end{cases}
$$

with $\eta \in \Omega_{k-\mu\left(O_{a^{\prime}}\right), U(1)}\left(O_{a^{\prime}}\right)$. The maps $e_{a^{\prime}}^{+}$and $e_{a}^{-}$are the end point maps for the moduli space $\mathcal{M}\left(O_{a^{\prime}}, O_{a}\right)$ of solutions on $\left(Y \times \mathbb{R}, \tilde{g}_{t}+d t^{2}\right)$.

The statement of the theorem now follows if we show that there is a chain homotopy $H$ such that

$$
i d_{k}-(J I)_{k}=D_{k+1} H_{k}+H_{k-1} D_{k} .
$$

In order to define $H$ let us consider the manifold $Y \times \mathbb{R}$ endowed with the metric $\gamma_{1}$ which is

$$
\gamma_{1}=\left\{\begin{array}{lr}
g_{0}+d t^{2} & t<-2 \\
g_{t+2}+d t^{2} & t \in[-2,-1] \\
g_{1}+d t^{2} & t \in[-1,1] \\
\tilde{g}_{2-t}+d t^{2} & t \in[1,2] \\
g_{0}+d t^{2} & t>2 .
\end{array}\right.
$$

Consider a path of metrics $\gamma_{\sigma}$ with $\sigma \in[0,1]$ that connects $\gamma_{0}=g_{0}+d t^{2}$ to $\gamma_{1}$, such that for all $\sigma$ the metric $\gamma_{\sigma}$ is the product metric $g_{0}+d t^{2}$ outside a fixed large interval $[-T, T]$.

Let $\mathcal{M}^{P}\left(O_{a}, O_{b}\right)$ be the parameterized moduli space of $(A(t), \psi(t), \sigma)$, solutions of the perturbed gradient flow equations with respect to the metric $\gamma_{\sigma}$, modulo gauge transformations. Lemma 6.5 , with minor modifications to accommodate the presence of parameters, can be employed to show that, under a generic choice of the perturbation, the moduli spaces $\mathcal{M}^{P}\left(O_{a}, O_{b}\right)$ are smooth manifolds of dimension $\mu\left(O_{a}\right)-\mu\left(O_{b}\right)+2-\operatorname{dim} G_{a}$, cut out transversely by the equations. Denote by $\tilde{e}_{a}^{+}$and $\tilde{e}_{b}^{-}$the end point maps from $\mathcal{M}^{P}\left(O_{a}, O_{b}\right)$ to $O_{a}$ and $O_{b}$ respectively.

Now we can define the degree-one map $H$ to be

$$
H: C_{k U(1)}\left(Y, g_{0}, \nu_{0}\right) \rightarrow C_{k+1 U(1)}\left(Y, g_{0}, \nu_{0}\right)
$$




$$
H_{a, b}: \quad \eta \rightarrow(-1)^{1-r(\eta)}\left(\tilde{e}_{b}^{-}\right)_{*}\left(\tilde{e}_{a}^{+}\right)^{*} \eta,
$$

with $\eta \in \Omega_{k-\mu\left(O_{a}\right), U(1)}\left(O_{a}\right)$.

Again we need to verify some properties of the compactified moduli spaces $\mathcal{M}^{P}\left(O_{a}, O_{b}\right)^{*}$, which ensure that the map $H$ is well defined and has the desired properties.

The identity (80) which proves that $H$ is a chain homotopy can be rewritten as the following two identities:

$$
\begin{aligned}
& i d_{a, a}-\sum_{a^{\prime}} I_{a, a^{\prime}} J_{a^{\prime}, a} \\
= & \sum_{\left\{b \mid \mu\left(O_{b}\right) \leq \mu\left(O_{a}\right)\right\}} D_{a, b} H_{b, a}+\sum_{\left\{b \mid \mu\left(O_{b}\right) \geq \mu\left(O_{a}\right)\right\}} H_{a, b} D_{b, a}
\end{aligned}
$$

and, for $a \neq b$,

$$
\begin{aligned}
& -\sum_{a^{\prime}} I_{a, a^{\prime}} J_{a^{\prime}, b} \\
= & \sum_{\left\{c \mid \mu\left(O_{c}\right) \leq \mu\left(O_{a}\right)\right\}} D_{a, c} H_{c, b}+\sum_{\left\{c \mid \mu\left(O_{c}\right) \geq \mu\left(O_{a}\right)\right\}} H_{a, c} D_{c, b} .
\end{aligned}
$$

The identities (83) and (84) can be proved by applying Stokes theorem again, in the way we discussed already. To this purpose, consider the components

$$
\begin{gathered}
\partial^{(1)} \mathcal{M}^{P}\left(O_{a}, O_{a}\right)^{*}=\bigcup_{a^{\prime}}\left(\mathcal{M}\left(O_{a}, O_{a^{\prime}}\right)^{*} \times O_{a^{\prime}} \mathcal{M}\left(O_{a^{\prime}}, O_{a}\right)^{*}\right) \cup\left\{-O_{a}\right\} \\
\bigcup_{\left\{b \mid \mu\left(O_{b}\right) \geq \mu\left(O_{a}\right)\right\}}\left((-1)^{\operatorname{dim} \hat{\mathcal{M}}\left(O_{b}, O_{a}\right)} \mathcal{M}^{P}\left(O_{a}, O_{b}\right)^{*} \times O_{b} \hat{\mathcal{M}}\left(O_{b}, O_{a}\right)^{*}\right) \\
\bigcup_{\left\{b \mid \mu\left(O_{b}\right) \leq \mu\left(O_{a}\right)\right\}}\left((-1)^{\operatorname{dim} \mathcal{M}^{P}\left(O_{b}, O_{a}\right)} \hat{\mathcal{M}}\left(O_{a}, O_{b}\right)^{*} \times O_{b} \mathcal{M}^{P}\left(O_{b}, O_{a}\right)^{*}\right)
\end{gathered}
$$

and, for $a \neq b$,

(86)

$$
\begin{aligned}
& \partial^{(1)} \mathcal{M}^{P}\left(O_{a}, O_{b}\right)^{*}=\bigcup_{a^{\prime}}\left(\mathcal{M}\left(O_{a}, O_{a^{\prime}}\right)^{*} \times_{O_{a^{\prime}}} \mathcal{M}\left(O_{a^{\prime}}, O_{b}\right)^{*}\right)
\end{aligned}
$$

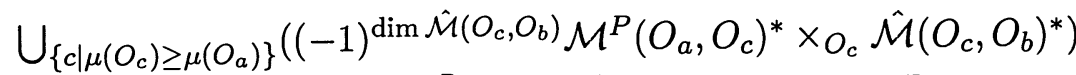

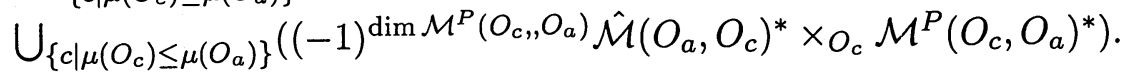

We need to know that these components are precisely the actual co-dimension one boundary in the compactifications $\mathcal{M}^{P}\left(O_{a}, O_{a}\right)^{*}$ and $\mathcal{M}^{P}\left(O_{a}, O_{b}\right)^{*}$ respectively. If we prove that the compactification $\mathcal{M}^{P}\left(O_{a}, O_{b}\right)^{*}$ has the structure of a smooth manifold with corners, with the actual boundary strata in the codimension one boundary consisting precisely of the components of (85) and (86), then the argument is complete.

Again, since we are dealing only with the model case of same chamber metrics and perturbations, the necessary properties of the compactification 
$\mathcal{M}^{P}\left(O_{a}, O_{b}\right)^{*}$ follow from the analysis introduced in Section 4, as in the case of moduli spaces of flow lines. This is another crucial point that requires a much more accurate analysis in the general case of different chambers. The signs in the formulae (78), (85), and (86) denote the difference of orientation, as in Theorem 6.9 and Theorem 6.10 .

In particular, we can describe more explicitly the components of the maps $I$ and $H$. This identifies what are, in fact, the minimal requirements on the compactifications of the moduli spaces $\mathcal{M}\left(O_{a}, O_{a^{\prime}}\right)$ and $\mathcal{M}^{P}\left(O_{a}, O_{b}\right)$ that are necessary for the argument given above to work.

Let $\eta_{a}$ be the one-form that generates $H^{1}\left(S^{1}\right)$ and $1_{a}$ be the constant function equal to 1 on $O_{a}$, so that we can write the generators of the equivariant complex in the form $\Omega^{n} \otimes \eta_{a}$ and $\Omega^{n} \otimes 1_{a}$. We have the following explicit description of the maps $I$ and $H$.

Remark 6.2. The components of the map $I$ vanish whenever we have $\mu\left(O_{a}\right)-\mu\left(O_{a^{\prime}}\right) \geq 2$. Moreover, the only possibly non-trivial components of the chain map $I$ are

$$
n_{a a^{\prime}}=\left\langle\Omega^{n} \otimes \eta_{a^{\prime}}, I\left(\Omega^{n} \otimes \eta_{a}\right)\right\rangle
$$

and

$$
n_{a a^{\prime}}=\left\langle\Omega^{n} \otimes 1_{a^{\prime}}, I\left(\Omega^{n} \otimes 1_{a}\right)\right\rangle,
$$

when $\mu\left(O_{a}\right)-\mu\left(O_{a^{\prime}}\right)=0$, with $O_{a}$ and $O_{a^{\prime}}$ free $U(1)$-orbits. In this case we need to know that the moduli space $\mathcal{M}\left(O_{a}, O_{a^{\prime}}\right)$ has a nice compactification $\mathcal{M}\left(O_{a}, O_{a^{\prime}}\right)^{*}$ analogous to the compactification of the moduli spaces of flow lines analyzed in Sections 4.2 and 4.3. We then have

$$
n_{a a^{\prime}}=\#\left(\mathcal{M}\left(a, a^{\prime}\right)=\mathcal{M}\left(O_{a}, O_{a^{\prime}}\right) / U(1)\right) .
$$

We also have

$$
m_{a b^{\prime}}=\left\langle\Omega^{n} \otimes 1_{b^{\prime}}, I\left(\Omega^{n} \otimes \eta_{a}\right)\right\rangle,
$$

when $\mu\left(O_{a}\right)-\mu\left(O_{b^{\prime}}\right)=1$ and with $O_{a}$ and $O_{b^{\prime}}$ free $U(1)$-orbits. In this case we need to know that the compactification $\mathcal{M}\left(O_{a}, O_{b^{\prime}}\right)^{*}$ is obtained by adding the co-dimension one boundary strata (89) and satisfies the analogue of Theorem 4.9, hence the push-forward map $\left(e_{b^{\prime}}^{-}\right)_{*}$ is well defined. Moreover, in the case of the critical points $\theta_{0}$ and $\theta_{1}$ we have

$$
n_{a, \theta_{1}}=\left\langle\Omega^{n} \otimes \theta_{1}, I\left(\Omega^{n} \otimes \eta_{a}\right)\right\rangle
$$


when $\mu\left(O_{a}\right)-\mu\left(\theta_{1}\right)=0$. The coefficient in this case is obtained by integrating along the 1-dimensional fibers of the map $e_{\theta_{1}}: \mathcal{M}\left(O_{a}, \theta_{1}\right) \rightarrow \theta_{1}$. Thus, we need to know that the moduli space $\mathcal{M}\left(O_{a}, \theta_{1}\right)$ is compact. This gives

$$
n_{a, \theta_{1}}=\# \mathcal{M}\left(a, \theta_{1}\right) .
$$

We also have the component

$$
n_{\theta_{0}, b^{\prime}}=\left\langle\Omega^{n} \otimes 1_{b^{\prime}}, I\left(\Omega^{n} \otimes \theta_{0}\right)\right\rangle,
$$

when $\mu\left(\theta_{0}\right)-\mu\left(O_{b^{\prime}}\right)=1$. Here we have a 1-dimensional space $\mathcal{M}\left(\theta_{0}, O_{b^{\prime}}\right)$ fibering over $O_{b^{\prime}}$ with zero-dimensional fiber, and again we need to know that we have $\mathcal{M}\left(\theta_{0}, O_{b^{\prime}}\right)^{*}=\mathcal{M}\left(\theta_{0}, O_{b^{\prime}}\right)$ in this case, which gives

$$
n_{\theta_{0}, b^{\prime}}=\# \mathcal{M}\left(\theta_{0}, b^{\prime}\right) \text {. }
$$

Remark 6.3. The components of the map $H$ vanish whenever we have $\mu\left(O_{a}\right)-\mu\left(O_{b}\right) \geq 1$. Moreover, the only possibly non-trivial components of the chain homotopy $H$ are

$$
n_{a b}^{P}=\left\langle\Omega^{n} \otimes \eta_{b}, H\left(\Omega^{n} \otimes \eta_{a}\right)\right\rangle,
$$

and

$$
n_{a b}^{P}=\left\langle\Omega^{n} \otimes 1_{b}, H\left(\Omega^{n} \otimes 1_{a}\right)\right\rangle,
$$

when $\mu\left(O_{a}\right)-\mu\left(O_{b}\right)=-1$, with $O_{a}$ and $O_{b}$ free $U(1)$-orbits. In this case we need to know that the one-dimensional moduli space $\mathcal{M}^{P}\left(O_{a}, O_{b}\right)$ is compact. This gives

$$
n_{a b}^{P}=\#\left(\mathcal{M}^{P}(a, b)=\mathcal{M}^{P}\left(O_{a}, O_{b}\right) / U(1)\right) .
$$

We also have

$$
m_{a c}^{P}=\left\langle\Omega^{n} \otimes 1_{c}, H\left(\left(\Omega^{n} \otimes \eta_{a}\right)\right\rangle,\right.
$$

when $\mu\left(O_{a}\right)-\mu\left(O_{c}\right)=0$ and with $O_{a}$ and $O_{c}$ free $U(1)$-orbits.

In this case we need to know that the compactification $\mathcal{M}^{P}\left(O_{a}, O_{c}\right)^{*}$ of the 2-dimensional moduli space $\mathcal{M}^{P}\left(O_{a}, O_{c}\right)$ is obtained by adding the codimension one boundary strata (86) and satisfies the analogue of Theorem 4.9 , hence the push-forward map $\left(\tilde{e}_{c}^{-}\right)_{*}$ used in the definition of $H$ is well defined. Moreover, in the case of the critical point $\theta_{0}$

$$
n_{a, \theta_{0}}^{P}=\left\langle\Omega^{n} \otimes \theta_{0}, H\left(\Omega^{n} \otimes \eta_{a}\right)\right\rangle,
$$


when $\mu\left(O_{a}\right)-\mu\left(\theta_{0}\right)=-1$. In this case the moduli space $\mathcal{M}^{P}\left(O_{a}, \theta_{0}\right)$ is one-dimensional.

Thus, in this case, the coefficient of $H$ is obtained by integrating along the 1-dimensional fibers of the map $e_{\theta_{0}}: \mathcal{M}^{P}\left(O_{a}, \theta_{0}\right)^{*} \rightarrow \theta_{0}$. Thus, we need to know that the 1-dimensional moduli space $\mathcal{M}^{P}\left(O_{a}, \theta_{0}\right)$ has a nice compactification $\mathcal{M}^{P}\left(O_{a}, \theta_{0}\right)^{*}$. This gives

$$
n_{a, \theta_{0}}^{P}=\# \mathcal{M}^{P}\left(a, \theta_{0}\right) \text {. }
$$

We also have the component

$$
n_{\theta_{0}, b}^{P}=\left\langle\Omega^{n} \otimes 1_{b}, H\left(\Omega^{n} \otimes \theta_{0}\right)\right\rangle,
$$

when $\mu\left(\theta_{0}\right)-\mu\left(O_{b}\right)=0$. Here we have a one-dimensional space $\mathcal{M}^{P}\left(\theta_{0}, O_{b}\right)$ fibering over $O_{b}$ with zero-dimensional fiber, and again we need to know that $\mathcal{M}^{P}\left(\theta_{0}, O_{b}\right)^{*}$ is a nice compactification. This gives

$$
n_{\theta_{0}, b}^{P}=\# \mathcal{M}^{P}\left(\theta_{0}, b\right) \text {. }
$$

This gives a fair description of the model argument in the easy case of metric and perturbations in the same chamber, and of the minimal requirements on the compactifications, in order to extend the argument to the more general case. Now we can begin to analyze the general case.

\subsection{The boundary structure of $\mathcal{M}\left(O_{a}, O_{a^{\prime}}\right)$.}

In this section we analyze some properties of the moduli spaces $\mathcal{M}\left(O_{a}, O_{a^{\prime}}\right)$ that are needed in the proof of Theorem 6.1. Here we make no assumption about the metrics and perturbations $\left(g_{0}, \nu_{0}\right),\left(g_{1}, \nu_{1}\right)$. We shall soon restrict our attention to the case of two sufficiently close metrics and perturbations on opposite sides of a codimension one wall, which is the only case we need to complete the proof of Theorem 6.1.

Let us first give a more precise account of the construction of the moduli spaces $\mathcal{M}\left(O_{a}, O_{a^{\prime}}\right)$. On $\left(Y \times \mathbb{R}, g_{t}+d t^{2}\right)$ we consider the equations

$$
D_{\mathbb{A}} \Psi+\rho \Psi=0
$$

and

$$
F_{\mathbb{A}}^{+}=\Psi \cdot \bar{\Psi}+i \mu+P_{(\mathbb{A}, \Psi)} .
$$

Here the form $\mu$ is determined by the path $\nu_{t}$ of perturbations, and the additional perturbation $P$ satisfies conditions (2)-(5) of Definition 2.10 and the modified version of condition (1): 
$\left(1^{\prime}\right)$ the perturbation $P_{(\mathbb{A}, \Psi)}$ restricted to the interval $(1, \infty)$ can be written as $P_{(\mathbb{A}, \Psi)}=* q_{(\mathbb{A}, \Psi)}(t)+q_{(\mathbb{A}, \Psi)}(t) \wedge d t$ with respect to the constant metric $g_{1}$, where $q_{(\mathbb{A}, \Psi)}(t)$ satisfies

$$
q_{\left.(\mathbb{A}, \Psi)\right|_{(1, \infty)} ^{T}}(t)=q_{(\mathbb{A}, \Psi)}(t+T)
$$

for any $T>0$ and for any $t>1$. Correspondingly, when restricted to the interval $(-\infty, 0)$ the perturbation $q_{(\mathbb{A}, \Psi)}(t)$, defined with respect to the constant metric $g_{0}$, satisfies

$$
q_{\left.(\mathbb{A}, \Psi)\right|_{(-\infty, 0)} ^{T}}(t)=q_{(\mathbb{A}, \Psi)}(t+T)
$$

for all $T<0$ and $t<0$.

In the Dirac equation (87), we add the perturbation term given by a 1 form $\rho$ on $Y \times \mathbb{R}$ that is supported on some compact set $Y \times\left[-T_{0}, T_{1}\right]$, with $T_{0}>0$ and $T_{1}>1$. Notice that, according to condition ( $\left.1^{\prime}\right)$, the equations (87) and (88) are translation invariant outside the interval $\left[-T_{0}, T_{1}\right]$. The moduli space $\mathcal{M}\left(O_{a}, O_{a^{\prime}}\right)$ is defined as the set of gauge equivalence classes of solutions of (87) and (88) in $\mathcal{B}_{k, \delta}^{0}\left(O_{a}, O_{a^{\prime}}\right)$.

Now, we first proceed as in Section 4.1 and prove the existence of a compactification for $\mathcal{M}\left(O_{a}, O_{a^{\prime}}\right)$. Theorem 6.4 (the analogue of Theorem 4.1) shows the existence of a compactification and identifies the ideal boundary with fibered products of lower dimensional moduli spaces, as in (89) and (90). This convergence argument follows very closely the argument for Theorem 4.1 , whereas the gluing arguments will require a more sophisticated analysis.

Theorem 6.4. Suppose either $O_{a}$ or $O_{a^{\prime}}$ is an irreducible critical orbit. Consider the moduli space $\mathcal{M}\left(O_{a}, O_{a^{\prime}}\right)$, with $\mu\left(O_{a}\right)-\mu\left(O_{a^{\prime}}\right)+\operatorname{dim}\left(O_{a}\right) \geq 1$. The codimension-one boundary of $\mathcal{M}\left(O_{a}, O_{a^{\prime}}\right)$ is a subset of the space

$$
\bigcup_{O_{b}} \hat{\mathcal{M}}\left(O_{a}, O_{b}\right) \times_{O_{b}} \mathcal{M}\left(O_{b}, O_{a^{\prime}}\right) \cup \bigcup_{O_{b^{\prime}}} \mathcal{M}\left(O_{a}, O_{b^{\prime}}\right) \times_{O_{b^{\prime}}} \hat{\mathcal{M}}\left(O_{b^{\prime}}, O_{a^{\prime}}\right) .
$$

Similarly, the components of higher codimension in the ideal boundary consist of fibered products of the form

$$
\begin{gathered}
\bigcup_{c_{1}, \cdots, c_{k}, b} \hat{\mathcal{M}}\left(O_{a}, O_{c_{1}}\right) \times O_{c_{1}} \cdots \\
\times_{O_{c_{k}}} \hat{\mathcal{M}}\left(O_{c_{k}}, O_{b}\right) \times_{O_{b}} \mathcal{M}\left(O_{b}, O_{a^{\prime}}\right) \\
\bigcup_{b^{\prime}, c_{1}^{\prime}, \cdots, c_{\ell}^{\prime}} \mathcal{M}\left(O_{a}, O_{b^{\prime}}\right) \times \times_{O_{b^{\prime}}} \hat{\mathcal{M}}\left(O_{b^{\prime}}, O_{c_{1}^{\prime}}\right) \times_{O_{c_{1}^{\prime}}} \cdots \\
\times_{O_{c_{\ell}^{\prime}}} \hat{\mathcal{M}}\left(O_{c_{\ell}^{\prime}}, O_{a^{\prime}}\right) .
\end{gathered}
$$


Proof. As in the proof of Theorem 4.1, we want to show that the space is precompact. Namely, any sequence $x_{i}$ of elements in $\mathcal{M}\left(O_{a}, O_{a^{\prime}}\right)$ has a subsequence which either converges in norm to another solution $x \in \mathcal{M}\left(O_{a}, O_{a^{\prime}}\right)$, or converges to a broken trajectory.

Given a sequence $x_{i}$ in $\mathcal{M}\left(O_{a}, O_{a^{\prime}}\right)$, we can follow the steps of the argument given in Theorem 4.1.

Claim 1. There is a subsequence $\left\{x_{i_{k}}\right\}$ that converges smoothly on compact sets to a finite energy solution $y$ of the perturbed equations (87) and (88) on $\left(Y \times \mathbb{R}, g_{t}+d t^{2}\right)$.

The proof of Claim 1 can be divided in the two cases of a compact set contained in the complement of $Y \times[0,1]$, where the metric is constant, and of the set $Y \times[0,1]$ where the metric changes. The first case is a direct application of the proof given in Theorem 4.1. In the second case, we obtain convergence as in [31], Lemma 3.19. We can find a sequence of gauge transformations $\lambda_{i}$ such that the forms $\lambda_{i}\left(\mathbb{A}_{i}-\mathbb{A}_{0}\right)$ are co-closed and annihilate normal vectors at the boundary. Lemma 4 of [30] then shows that the sequence $\lambda_{i}\left(\mathbb{A}_{i}, \Psi_{i}\right)$ has a subsequence that converges smoothly on $Y \times[0,1]$.

Notice that the notion of finite energy, as well as the results of Corollary 3.11 and Theorem 3.12, extend to the case of solutions on $\left(Y \times \mathbb{R}, g_{t}+d t^{2}\right)$, since the metric is constant outside the compact set $Y \times[0,1]$. This implies that the argument given in Theorem 4.1 proves the following claim as well.

Claim 2. If the limit $y$ is an element of $\mathcal{M}\left(O_{a}, O_{a^{\prime}}\right)$ then the convergence $x_{i} \rightarrow y$ is strong in the $L_{k, \delta}^{2}$ norm.

Thus we need to check whether there are obstructions to the convergence in norm, that is, whether broken trajectories arise as limits.

Suppose the element $y$ is a limit smoothly on compact sets, but is not a limit in norm. Again, since the result of Corollary 3.11 holds, the solution $y$ defines an element in some moduli space $\mathcal{M}\left(O_{b}, O_{b^{\prime}}\right)$.

Since the metric is translation invariant only outside the interval $[0,1]$, consider the restriction of the sequence $x_{i}$ and of the limit $y$ to the interval $(1, \infty)$ with the constant metric $g_{1}$. We can choose a value $\alpha$ satisfying

$$
\mathcal{C}_{\rho}\left(O_{b^{\prime}}\right)>\alpha>\mathcal{C}_{\rho}\left(O_{a^{\prime}}\right)
$$

We can find times $T_{i}>1$ such that $\mathcal{C}_{\rho}\left(x_{i}\left(T_{i}\right)\right)=\alpha$. The restriction to $(1, \infty)$ of the reparameterized sequence $x_{i}^{T_{i}}$ is again a sequence of solutions of the flow equations with the constant metric $g_{1}$ on $Y \times(1, \infty)$. A subsequence 
converges smoothly on compact sets to a solution $\tilde{y}$. This defines an element in some $\mathcal{M}\left(O_{c^{\prime}}, O_{d^{\prime}}\right)$, subject to the constraint

$$
\mathcal{C}_{\rho}\left(O_{b^{\prime}}\right) \geq \mathcal{C}_{\rho}\left(O_{c^{\prime}}\right)>\mathcal{C}_{\rho}\left(O_{d^{\prime}}\right) \geq \mathcal{C}_{\rho}\left(O_{a^{\prime}}\right)
$$

Notice that the proof of Theorem 6.4 is essentially along the same lines as the proof of the analogous result, Theorem 4.1, for the moduli spaces of flow lines $\hat{\mathcal{M}}\left(O_{a}, O_{b}\right)$. If we could guarantee that the cokernels of the linearizations are always trivial, then we could also extend the results of Section 4.2 and 4.3 to this case, showing that the same properties of the compactification hold true. However, the essential new feature which appears in dealing with the moduli spaces $\mathcal{M}\left(O_{a}, O_{a^{\prime}}\right)$ is precisely that not all the cokernels are trivial. Thus, we need a much more accurate analysis of the fine structure of the compactification.

The strategy we follow is to restrict our attention to just the cases which are needed for the proof of Theorem 6.4, and show that, in those cases, we obtain the desired properties of the compactification. The purpose of the rest of Section 6 is to deal with these issues in detail.

In order to identify where the problem of cokernels may arise, we study the transversality issue for the moduli spaces $\mathcal{M}\left(O_{a}, O_{a}^{\prime}\right)$. The following Lemma shows that the appearance of non-trivial cokernels is confined to the case of the moduli space $\mathcal{M}\left(\theta_{0}, \theta_{1}\right)$ with $\mu\left(\theta_{0}\right)-\mu\left(\theta_{1}\right)<0$.

Lemma 6.5. Suppose given $\left(g_{0}, \nu_{0}\right),\left(g_{1}, \nu_{1}\right)$ and a generic path $\left(g_{t}, \nu_{t}\right)$ connecting them. Consider the manifold $Y \times \mathbb{R}$ with the metric $g_{t}+d t^{2}$, constant outside the interval $[0,1]$. Suppose given $x$, a solution of equations (87) and (88), with asymptotic values on the critical orbits $O_{a}$ and $O_{a^{\prime}}$. Consider the linearization $\mathcal{L}_{x}$ of equations (87) and (88). Under a generic choice of the metric and of the perturbations $P$ and $\rho$, we have the following result. If the solution $x=(\mathbb{A}, \Psi)$ satisfies $\Psi \neq 0$, then the linearization $\mathcal{L}_{x}$ is surjective. If we have $O_{a}=\theta_{0}$ and $O_{a^{\prime}}=\theta_{1}$ and the solution $x$ is of the form $x=(\mathbb{A}, 0)$, then the linearization $\mathcal{L}_{x}$ is surjective, provided that the relative Morse index satisfies $\mu\left(\theta_{0}\right)-\mu\left(\theta_{1}\right) \geq 0$.

The result of Lemma 6.5 can be rephrased as the following transversality statement for the moduli spaces $\mathcal{M}\left(O_{a}, O_{a}^{\prime}\right)$.

Lemma 6.6. Consider the following cases:

(a) at least one of the asymptotic critical orbits $O_{a}$ and $O_{a^{\prime}}$ has a free $U(1)$-action. 
(b) We have $O_{a}=\theta_{0}$ and $O_{a^{\prime}}=\theta_{1}$ under the condition that $\mu\left(\theta_{0}\right)-\mu\left(\theta_{1}\right) \geq$ 0 .

Then, for a generic choice of the metric and perturbations, the moduli space $\mathcal{M}\left(O_{a}, O_{a^{\prime}}\right)$ is a smooth oriented manifold that is cut out transversely by the equations, of dimension $\mu\left(O_{a}\right)-\mu\left(O_{a^{\prime}}\right)+\operatorname{dim}\left(O_{a}\right)$. In particular, in case (a) the moduli space is generically empty when the virtual dimension $\mu\left(O_{a}\right)-\mu\left(O_{a^{\prime}}\right)+\operatorname{dim}\left(O_{a}\right)$ is negative.

Proof of Lemma 6.5 and 6.6. No solution with $\Psi \equiv 0$ arises under the hypothesis of case (a). Thus the argument of Proposition 2.14 and Proposition 3.14 , applied to the linearization

$$
\mathcal{L}_{(\mathbb{A}, \Psi, P, \rho)}(\alpha, \Phi)=\left\{\begin{array}{c}
D_{\mathbb{A}} \Phi+\alpha \Psi+\rho \Phi \\
d^{+} \alpha-\frac{1}{2} \operatorname{Im}(\Psi \cdot \bar{\Phi})+\mathcal{D} P_{(\mathbb{A}, \Psi)}(\alpha, \Phi) \\
G_{(\mathbb{A}, \Psi)}^{*}(\alpha, \Phi)
\end{array}\right.
$$

gives the desired transversality result. In case (b), we can find solutions in $\mathcal{M}\left(\theta_{0}, \theta_{1}\right)$ with $\Psi \neq 0$, as well as a solution with $\Psi \equiv 0$. The linearization is surjective at solutions with $\Psi \neq 0$ because of the argument of Proposition 2.14 and Proposition 3.14. Consider the linearization $\mathcal{L}_{x}$ at a solution of the form $x=(\mathbb{A}, 0)$. We have

$$
\mathcal{L}_{(\mathbb{A}, 0, P, \rho)}(\alpha, \Phi)=\left\{\begin{array}{c}
D_{\mathbb{A}} \Phi+\rho \Phi \\
d^{+} \alpha+\mathcal{D} P_{(\mathbb{A}, 0)}(\alpha, \Phi) \\
G_{(\mathbb{A}, 0)}^{*}(\alpha, \Phi)
\end{array}\right.
$$

Consider the operator

$$
\hat{\mathcal{L}}_{(\mathbb{A}, 0, P, \rho)}(\alpha, \Phi, p, \eta)=\mathcal{L}_{(\mathbb{A}, 0, P)}(\alpha, \Phi)+\left(\begin{array}{c}
\eta \Phi \\
p_{(\mathbb{A}, 0, P)}(\alpha, \Phi)
\end{array}\right)
$$

where we vary the perturbation of the curvature equation by an element $p_{(\mathbb{A}, 0, P)}$ of $T_{P} \mathcal{P}$, and the perturbation of the Dirac equation by a compactly supported 1-form $\eta$. We follow the analogous argument of Proposition 2.14. Suppose $(\beta, \xi)$ is an element orthogonal to the range of $\hat{\mathcal{L}}_{(\mathbb{A}, 0, P, \rho)}$. We have terms of the form

$$
\begin{aligned}
\left\langle\beta, d^{+} \alpha+\right. & \left.\mathcal{D} P_{(\mathbb{A}, 0)}(\alpha, \Phi)+p_{(\mathbb{A}, 0, P)}(\alpha, \Phi)\right\rangle \\
& +\left\langle\xi, D_{\mathbb{A}} \Phi+\eta \cdot \Phi\right\rangle=0 .
\end{aligned}
$$

By varying $p \in \mathcal{P}$ we force $\beta \equiv 0$. If $\mu\left(\theta_{0}\right)-\mu\left(\theta_{1}\right)=0$, then the condition

$$
\left\langle\xi, D_{\mathbb{A}} \Phi+\eta \cdot \Phi\right\rangle=0
$$


implies that $\xi$ is in the kernel of $D_{\mathbb{A}-\eta}$, but for a generic choice of $\eta$ this operator has trivial kernel. If $\mu\left(\theta_{0}\right)-\mu\left(\theta_{1}\right)>0$, the argument is similar: choose $\Phi$ to be a non-trivial element in $\operatorname{Ker}\left(D_{\mathbb{A}}\right)$. This is possible since we are assuming that $\mu\left(\theta_{0}\right)-\mu\left(\theta_{1}\right)>0$, hence that $\operatorname{dim} \operatorname{Ker}\left(\mathcal{L}_{x}\right)>0$. We show that, by varying $\eta$ we force $\xi$ to vanish. In fact, suppose

$$
\langle\xi, \eta \cdot \Phi\rangle=0
$$

is satisfied for all possible compactly supported 1-forms $\eta$, and for a nontrivial $\xi$. Then we can follow the argument of [45] 6.2.1: choose some small open set where both $\Phi$ and $\xi$ are non-trivial and approximately constant. The isomorphism

$$
\mathbb{R}^{4} \otimes_{\mathbb{R}} \mathbb{C} \rightarrow \operatorname{Hom}_{\mathbb{C}}\left(S^{+}, S^{-}\right)
$$

induced by Clifford multiplication implies that there exist $\eta \in \mathbb{R}^{4}$ such that

$$
\operatorname{Re}(\langle\xi, \eta \cdot \Phi\rangle)>0
$$

at some point in the small open set $U$. By a trivialization of $T^{*}(Y \times R)$ on $U$ the vector $\eta$ can be extended to a 1 -form $\eta$ on $U$. This 1 -form can be extended to a compactly supported 1-form in a neighborhood of $U$, so that we get

$$
\int_{Y \times \mathbb{R}} \operatorname{Re}(\langle\xi, \eta \cdot \Phi\rangle)>0,
$$

which contradicts the initial assumption.

Notice that, since we consider the gauge group $\mathcal{G}^{0}\left(O_{a}, O_{a^{\prime}}\right)$ of gauge transformations that decay to elements $\lambda( \pm \infty)$ in the stabilizers $G_{a}$ and $G_{a^{\prime}}$, a constant element $g$ is necessarily trivial, hence the element $x=(\mathbb{A}, 0)$ is a manifold point in $\mathcal{B}^{0}\left(O_{a}, O_{a^{\prime}}\right)$, fixed by the $U(1)$-action. Thus, the operator $\mathcal{L}_{(\mathbb{A}, \Psi, P, \rho)}$ is surjective, for a generic choice of the perturbations $P$ and $\rho$. This proves that in case (a) or case (b) the moduli space $\mathcal{M}\left(O_{a}, O_{a^{\prime}}\right)$ is cut out transversely by the equations and of dimension equal to the virtual dimension prescribed by the index theorem. The orientation of the moduli spaces $\mathcal{M}\left(O_{a}, O_{a^{\prime}}\right)$ is obtained as in Proposition 2.15, from an orientation of the homology groups

$$
H_{\delta}^{0}(Y \times \mathbb{R}) \oplus H_{\delta}^{2+}(Y \times \mathbb{R}) \oplus H_{\delta}^{1}(Y \times \mathbb{R}) .
$$

Notice that, since we are dealing with the case of homology spheres, we have only finitely many critical orbits $O_{a}$ and $O_{a^{\prime}}$. Thus we can assume that 
there is a fixed compact set $Y \times\left[-T_{0}, T_{1}\right]$, with $T_{0} \geq 0$ and $T_{1} \geq 1$, such that transversality of all the moduli spaces of case (a) and (b) of Lemma 6.6 can be achieved by a perturbation $\rho$ supported on $Y \times\left[-T_{0}, T_{1}\right]$, together with the perturbation $P$ of the curvature equation.

Notice that outside $\left[-T_{0}, T_{1}\right]$ the metric is translation invariant and the equations coincide with the gradient flow equations. For simplicity of notation in the following we shall assume that $\left[-T_{0}, T_{1}\right]$ is just the interval $[0,1]$.

We have the following result.

Corollary 6.7. If either $O_{b}$ or $O_{b^{\prime}}$ is a critical orbit with a free $U(1)$-action, then there are no reducible flow lines in the moduli space $\mathcal{M}\left(O_{b}, O_{b^{\prime}}\right)$, and the moduli space is generically empty if the virtual dimension is negative.

Consider instead the case $O_{a}=\theta_{0}$ and $O_{a^{\prime}}=\theta_{1}$ with $\mu\left(\theta_{0}\right)-\mu\left(\theta_{1}\right)<0$. In this case no solution with $\Psi \neq 0$ appears in the moduli space $\mathcal{M}\left(\theta_{0}, \theta_{1}\right)$.

Both statements are consequences of Lemma 6.5. In fact, if there were any solution, then by argument of Lemma 6.6 the linearization ought to be surjective for a generic choice of the perturbations, and this is incompatible with the value of the index.

This result shows that reducible solutions (non-smooth points) in $\mathcal{M}\left(O_{a}, O_{a^{\prime}}\right)$ may appear only in the moduli spaces $\mathcal{M}\left(\theta_{0}, \theta_{1}\right)$, with $\mu\left(\theta_{0}\right)$ $\mu\left(\theta_{1}\right)<0$, which is in fact the only case not covered by the transversality result of Lemma 6.5 .

We can give a more precise description of this moduli spaces as follows.

Lemma 6.8. Consider a choice of metrics and perturbations $\left(g_{0}, \nu_{0}\right)$ and $\left(g_{1}, \nu_{1}\right)$ in different chambers, with a negative spectral flow $S F\left(\partial_{\nu_{t}}^{g_{t}}\right)<0$. In this case, the moduli space $\mathcal{M}\left(\theta_{0}, \theta_{1}\right)$ consists of a unique reducible class $x=$ $[\mathbb{A}, 0]$. In particular, $\mathcal{M}\left(\theta_{0}, \theta_{1}\right)$ is non-empty, though the virtual dimension $\mu\left(\theta_{0}\right)-\mu\left(\theta_{1}\right)<0$ is negative. Thus, we have

$$
\operatorname{dim} \operatorname{Coker}\left(\mathcal{L}_{x}\right)=\left|\mu\left(\theta_{0}\right)-\mu\left(\theta_{1}\right)\right|=-S F\left(\partial_{\nu_{t}}^{g_{t}}\right) .
$$

Proof. Lemma 6.6 shows that $\mathcal{M}\left(\theta_{0}, \theta_{1}\right)$ can only contain reducible solutions. In particular, $x=[\mathbb{A}, 0]$ is obtained as the unique solution of the equations

$$
d^{+} \mathbb{A}=\mu \text { and } d^{*} \mathbb{A}=0 .
$$

Here $d^{*}$ is the operator on the four-manifold $Y \times \mathbb{R}$, with $*_{4}$ the Hodge operator with respect to the metric $g_{t}+d t^{2}$, and the perturbation form $\mu$ is 
of the form $\mu=d \nu_{t}+*_{3} d \nu_{t} \wedge d t$, with the $*_{3}$ operator with respect to the metric $g_{t}$.

This solution of the four-dimensional Seiberg-Witten equations gives an element $x=[\mathbb{A}, 0]$ which is a fixed point of the $U(1)$-action on the configuration space $\mathcal{B}^{0}\left(\theta_{0}, \theta_{1}\right)$. Notice that, though the point $x=[\mathbb{A}, 0]$ is a smooth point in $\mathcal{B}^{0}\left(\theta_{0}, \theta_{1}\right)$ (fixed by the $U(1)$-action), it is a singular point in $\mathcal{M}\left(\theta_{0}, \theta_{1}\right)$, due to the lack of transversality, that is, the presence of a non-trivial Cokernel.

We see, from the previous discussion, that one first problem we encounter in extending the proof of the easy case of Theorem 6.1 to the harder case is the compactification of the moduli spaces $\mathcal{M}\left(O_{a}, O_{a^{\prime}}\right)$. In fact, consider the case of the compactification of a moduli space $\mathcal{M}\left(\theta_{0}, O_{a^{\prime}}\right)$, under the assumption that $\mu\left(\theta_{0}\right)-\mu\left(\theta_{1}\right)<0$.

For instance, in the case $\mu\left(\theta_{0}\right)-\mu\left(\theta_{1}\right)=-2$, by the result of Theorem 6.4 , we know that the product

$$
\mathcal{M}\left(\theta_{0}, \theta_{1}\right) \times \hat{\mathcal{M}}\left(\theta_{1}, O_{a^{\prime}}\right)
$$

is a possible component of the ideal boundary. The counting of the virtual dimensions is correct as prescribed by Theorem 6.4 for components of codimension one in the ideal boundary. In fact, we have

$$
\begin{gathered}
\operatorname{virtdim}\left(\mathcal{M}\left(\theta_{0}, \theta_{1}\right) \times \hat{\mathcal{M}}\left(\theta_{1}, O_{a^{\prime}}\right)\right)=\mu\left(\theta_{0}\right)-\mu\left(\theta_{1}\right)+\mu\left(\theta_{1}\right)-\mu\left(O_{a^{\prime}}\right)-1 \\
=\mu\left(\theta_{0}\right)-\mu\left(O_{a^{\prime}}\right)-1=\operatorname{dim}\left(\mathcal{M}\left(\theta_{0}, O_{a^{\prime}}\right)\right)-1 .
\end{gathered}
$$

However, from the result of Lemma 6.8, we know that the actual dimension of $\mathcal{M}\left(\theta_{0}, \theta_{1}\right)$ is zero, since it consists of the unique solution $x=(\mathbb{A}, 0)$, hence the actual dimension of (91) is equal to $\mu\left(\theta_{1}\right)-\mu\left(O_{a^{\prime}}\right)$. This gives

$$
\begin{aligned}
& \operatorname{dim}\left(\mathcal{M}\left(\theta_{0}, \theta_{1}\right) \times \hat{\mathcal{M}}\left(\theta_{1}, O_{a^{\prime}}\right)\right)=\mu\left(\theta_{1}\right)-\mu\left(O_{a^{\prime}}\right)-1 \\
& =\mu\left(\theta_{0}\right)+2-\mu\left(O_{a^{\prime}}\right)-1=\operatorname{dim}\left(\mathcal{M}\left(\theta_{0}, O_{a^{\prime}}\right)\right)+1 .
\end{aligned}
$$

Clearly, this implies that, if an entire component of the form (91) actually appears in the compactification (that is, if they can be spliced together and deformed to actual solutions as described in Section 4.2), then the resulting compactification $\mathcal{M}\left(\theta_{0}, O_{a^{\prime}}\right)^{*}$ does not have the structure of smooth manifold with corners described in Section 4.3. This could cause problems in applying the arguments based on Stokes' theorem in the proof of Theorem 6.1. Section 6.2 deals with this problem. The main result is stated in Theorem 6.21. 
We now proceed with general properties of the compactification of the moduli spaces $\mathcal{M}\left(O_{a}, O_{a^{\prime}}\right)$ and $\mathcal{M}^{P}\left(O_{a}, O_{b}\right)$ which we can derive in general, regardless of the behavior of the singular components of the ideal boundary.

We have the following gluing theorem.

Theorem 6.9. For $O_{a}$ and $O_{a^{\prime}}$ irreducible, given a compact set

$$
K \subset \hat{\mathcal{M}}\left(O_{a}, O_{b}\right) \times_{O_{b}} \mathcal{M}\left(O_{b}, O_{a^{\prime}}\right)
$$

or

$$
K \subset \mathcal{M}\left(O_{a}, O_{b^{\prime}}\right) \times_{O_{b^{\prime}}} \hat{\mathcal{M}}\left(O_{b^{\prime}}, O_{a^{\prime}}\right),
$$

there is a lower bound $T_{0}(K)>0$ and gluing maps

$$
\#: K \times[T, \infty) \rightarrow \mathcal{M}\left(O_{a}, O_{a^{\prime}}\right),
$$

such that $\#_{T}$ is a smooth embedding for all $T \geq T_{0}(K)$. The gluing maps have the property that any sequence of solutions in $\mathcal{M}\left(O_{a}, O_{a^{\prime}}\right)$ that converges to an element in the boundary is eventually contained in the range of the gluing map. The map \# is orientation preserving with respect to the orientation given by

$$
\left(\hat{\mathcal{M}}\left(O_{a}, O_{b}\right) \times \mathbb{R}^{+}\right) \times O_{b} \mathcal{M}\left(O_{b}, O_{a^{\prime}}\right)
$$

and

$$
\mathcal{M}\left(O_{a}, O_{b^{\prime}}\right) \times_{O_{b^{\prime}}}\left(\hat{\mathcal{M}}\left(O_{b^{\prime}}, O_{a^{\prime}}\right) \times \mathbb{R}^{+}\right)
$$

Similarly, for

$$
K \subset \hat{\mathcal{M}}\left(O_{a}, \theta_{0}\right) \times \mathcal{M}\left(\theta_{0}, O_{a^{\prime}}\right)
$$

or

$$
K \subset \mathcal{M}\left(O_{a}, \theta_{1}\right) \times \hat{\mathcal{M}}\left(\theta_{1}, O_{a^{\prime}}\right),
$$

there is a $T_{0}(K)>0$ and gluing maps

$$
\#: K \times[T, \infty) \rightarrow \mathcal{M}\left(O_{a}, O_{a^{\prime}}\right),
$$

which are smooth embeddings for $T \geq T_{0}(K)$, and with the same properties on the range and on the orientation.

Proof. We know from the results of Lemma 6.6 that all the moduli spaces involved are smooth manifolds, cut out transversely by the equations. In particular, for any element $(x, y)$ in a fibered product as above, the cokernels $\operatorname{Coker}\left(\mathcal{L}_{x}\right)$ and $\operatorname{Coker}\left(\mathcal{L}_{y}\right)$ are trivial. Thus, we can adapt the same 
argument used in Section 4.2 for the gluing construction, using the result of Proposition 4.21.

Namely, we proceed as follows. We show that all broken trajectories of the form $\mathcal{M}\left(O_{a}, O_{b^{\prime}}\right) \times_{O_{b}} \hat{\mathcal{M}}\left(O_{b^{\prime}}, O_{a^{\prime}}\right)$ are indeed contained in the boundary of $\mathcal{M}\left(O_{a}, O_{a^{\prime}}\right)$. The argument for the components of the form $\hat{\mathcal{M}}\left(O_{a}, O_{b}\right) \times_{O_{b}} \mathcal{M}\left(O_{b}, O_{a^{\prime}}\right)$ is analogous. We need to show that, given $x$ and $\hat{y}$ in $\mathcal{M}\left(O_{a}, O_{b^{\prime}}\right)$ and $\hat{\mathcal{M}}\left(O_{b^{\prime}}, O_{a^{\prime}}\right)$ respectively, we can form a pre-glued approximate solution and perturb it to an actual solution as in Theorem 4.9. We have temporal gauge representatives

$$
\left(A_{1}(t), \psi_{1}(t)\right) \in \mathcal{A}_{k, \delta}\left(O_{a}, O_{b^{\prime}}\right)
$$

and

$$
\left(A_{2}(t), \psi_{2}(t)\right) \in \mathcal{A}_{k, \delta}\left(O_{b^{\prime}}, O_{a^{\prime}}\right) .
$$

The solution $y$ is translation invariant, but the solution $x$ is not, since the metric is constant only outside the interval $[0,1]$. Thus we have to modify slightly the definition of the pre-gluing map. Choose slices

$$
\mathcal{S}_{\Gamma_{a b^{\prime}}} \subset \mathcal{A}_{k, \delta}\left(O_{a}, O_{b^{\prime}}\right)
$$

and

$$
\mathcal{S}_{\Gamma_{b^{\prime} a^{\prime}}} \subset \mathcal{A}_{k, \delta}\left(O_{b^{\prime}}, O_{a^{\prime}}\right)
$$

determined by the elements

$$
\Gamma_{a b^{\prime}} \in \mathcal{A}_{k, \delta}\left(\left(A_{a}, \psi_{a}\right),\left(A_{b^{\prime}}, \psi_{b^{\prime}}\right)\right)
$$

and

$$
\Gamma_{b^{\prime} a^{\prime}} \in \mathcal{A}_{k, \delta}\left(\left(A_{b^{\prime}}, \psi_{b^{\prime}}\right),\left(A_{a^{\prime}}, \psi_{a^{\prime}}\right)\right) .
$$

We choose them in such a way that we have representatives $x$ and $y$ in a ball of radius $r$ centered at $\Gamma_{a b^{\prime}}$ and $\Gamma_{b^{\prime} a^{\prime}}$ respectively. Then, according to Lemma 4.8, there are gauge transformations $\lambda_{1}^{+}$and $\lambda_{2}^{-}$that conjugate $x$ and $y$ into $W_{\Gamma_{a b^{\prime}}}^{+}$and $W_{\Gamma_{b^{\prime} a^{\prime}}}^{-}$respectively. For $t \geq T$, there is a gauge transformation $\lambda_{b^{\prime}} \in \mathcal{G}(Y)$ such that we can write $\lambda_{1}^{+} x(t)=\lambda_{b^{\prime}}\left(A_{b^{\prime}}, \psi_{b^{\prime}}\right)+\left(\alpha_{1}(t), \phi_{1}(t)\right)$ and $\lambda_{2}^{-} y(t)=\lambda_{b^{\prime}}\left(A_{b^{\prime}}, \psi_{b^{\prime}}\right)+\left(\alpha_{2}(t), \phi_{2}(t)\right)$, where $\left[A_{b^{\prime}}, \psi_{b^{\prime}}\right]$ is a point on the orbit $O_{b^{\prime}}$ and we have

$$
\lim _{t \rightarrow \infty}\left(A_{1}(t), \psi_{1}(t)\right)=\lambda_{b^{\prime}}\left(A_{b^{\prime}}, \psi_{b^{\prime}}\right)=\lim _{t \rightarrow-\infty}\left(A_{2}(t), \psi_{2}(t)\right) .
$$


The (right) pre-glued approximate solution is given as follows:

(94) $x \#_{T}^{0} y=\left\{\begin{array}{lr}\lambda_{1}^{+} x & t \leq T-1 \\ \lambda_{b^{\prime}}\left(A_{b^{\prime}}, \psi_{b^{\prime}}\right)+\rho_{T}^{-}(t)\left(\alpha_{1}(t), \phi_{1}(t)\right)+ & \\ \rho_{T}^{+}(t)\left(\alpha_{2}(t-2 T), \phi_{2}(t-2 T)\right) & T-1 \leq t \leq T+1 \\ \lambda_{2}^{-} y^{-2 T} & t \geq T+1 .\end{array}\right.$

Here $\rho_{T}^{ \pm}(t)$ are smooth cutoff functions with bounded derivative, such that $\rho_{T}^{-}(t)$ is equal to one for $t \leq T-1$ and to zero for $t \geq T$ and $\rho_{T}^{+}(t)$ is equal to zero for $t \leq T$ and to one for $t \geq T+1$. Lemma 4.10 shows that $\#_{T}^{0}$ descends to a well defined pre-gluing

$$
\#_{T}^{0}: \mathcal{M}\left(O_{a}, O_{b^{\prime}}\right) \times_{O_{b^{\prime}}} \hat{\mathcal{M}}\left(O_{b^{\prime}}, O_{a^{\prime}}\right) \rightarrow \mathcal{B}^{0}\left(O_{a}, O_{a^{\prime}}\right) .
$$

We are assuming that $O_{a}$ and $O_{a^{\prime}}$ have a free $U(1)$ action, thus, according to Lemma 6.6, Corollary 6.7, and Proposition 2.12, we can ensure that, for a generic choice of the metric and perturbation we have $\operatorname{Coker}\left(\mathcal{L}_{x}\right)=0$ and $\operatorname{Coker}\left(\mathcal{L}_{y}\right)=0$. Lemma 4.13 and Proposition 4.19 show that we have an isomorphism at the level of the actual kernel of the linearization of the pre-glued solution:

$$
\operatorname{Ker}\left(\mathcal{L}_{x \#_{T}^{0} y}\right) \cong \operatorname{Ker}\left(\mathcal{L}_{x}\right) \times \operatorname{Ker}\left(\mathcal{L}_{y}\right) .
$$

This implies that we have $\operatorname{Coker}\left(\mathcal{L}_{x \#_{T}^{0} y}\right)=0$ We proceed as in Proposition 4.21 and we are able to perturb the approximate solutions to an actual solutions by the fixed point argument of Theorem 4.9.

In the case of pre-gluing broken trajectories in the components

$$
\hat{\mathcal{M}}\left(O_{a}, O_{b}\right) \times_{O_{b}} \mathcal{M}\left(O_{b}, O_{a^{\prime}}\right)
$$

we need to consider the (left) pre-gluing map

$$
x \#_{-T}^{0} y=\left\{\begin{array}{lr}
\lambda_{1}^{+} x^{2 T} & t \leq-T-1 \\
\lambda_{b}\left(A_{b}, \psi_{b}\right)+\rho_{-T}^{+}(t)\left(\alpha_{2}(t), \phi_{2}(t)+\right. & \\
\rho_{-T}^{-}(t)\left(\alpha_{1}(t+2 T), \phi_{1}(t+2 T)\right) & -T-1 \leq t \leq-T+1 \\
\lambda_{2}^{-} y & t \geq-T+1 .
\end{array}\right.
$$

Here $\rho_{-T}^{ \pm}(t)$ are smooth cutoff functions with bounded derivative, such that $\rho_{-T}^{-}(t)$ is equal to one for $t \leq-T-1$ and to zero for $t \geq-T$ and $\rho_{-T}^{+}(t)$ is equal to zero for $t \leq-T$ and to one for $t \geq-T+1$. The rest of the argument is analogous. We have

$$
\operatorname{Ker}\left(\mathcal{L}_{x \#_{T}^{0} y}\right) \cong \operatorname{Ker}\left(\mathcal{L}_{x}\right) \times \operatorname{Ker}\left(\mathcal{L}_{y}\right)
$$


and Coker $\left(\mathcal{L}_{x \#_{T}^{0} y}\right)=0$. We then proceed as in the previous case.

We have a similar result for the moduli spaces $\mathcal{M}^{P}\left(O_{a}, O_{c}\right)$.

Theorem 6.10. For $O_{a}$ and $O_{c}$ free orbits, consider a compact set

$$
K \subset \mathcal{M}^{P}\left(O_{a}, O_{b}\right) \times_{O_{b}} \hat{\mathcal{M}}\left(O_{b}, O_{c}\right)
$$

or

$$
K \subset \hat{\mathcal{M}}\left(O_{a}, O_{b}\right) \times_{O_{b}} \mathcal{M}^{P}\left(O_{b}, O_{c}\right) .
$$

There is a bound $T_{0}(K)$ and gluing maps

$$
\#: K \times\left[T_{0}, \infty\right) \rightarrow \mathcal{M}^{P}\left(O_{a}, O_{c}\right)
$$

that are smooth embeddings, compatible with the orientation induced by the product orientation of

$$
\mathcal{M}^{P}\left(O_{a}, O_{b}\right) \times \mathbb{R} \times \hat{\mathcal{M}}\left(O_{b}, O_{c}\right)
$$

and

$$
\hat{\mathcal{M}}\left(O_{a}, O_{b}\right) \times \mathbb{R} \times \mathcal{M}^{P}\left(O_{b}, O_{c}\right) .
$$

Similarly, for a compact set

$$
K \subset \mathcal{M}\left(O_{a}, O_{a^{\prime}}\right) \times_{O_{a^{\prime}}} \mathcal{M}\left(O_{a^{\prime}}, O_{c}\right),
$$

there is a $T_{0}(K)$ and an orientation preserving gluing map

$$
\#: K \subset \mathcal{M}\left(O_{a}, O_{a^{\prime}}\right) \times_{O_{a^{\prime}}} \mathcal{M}\left(O_{a^{\prime}}, O_{c}\right) \rightarrow \mathcal{M}^{P}\left(O_{a}, O_{c}\right) \cap\{\sigma=1\}
$$

that is a smooth embedding. Since by construction the moduli space $\mathcal{M}^{P}\left(O_{a}, O_{c}\right)$ satisfies

$$
\mathcal{M}^{P}\left(O_{a}, O_{c}\right) \cap\{\sigma \in(1-\epsilon, 1]\} \cong\left(\mathcal{M}^{P}\left(O_{a}, O_{c}\right) \cap\{\sigma=1\}\right) \times(1-\epsilon, 1],
$$

this provides the necessary collar structure at the boundary components

$$
\mathcal{M}\left(O_{a}, O_{a^{\prime}}\right) \times_{O_{a^{\prime}}} \mathcal{M}\left(O_{a^{\prime}}, O_{c}\right) .
$$

Moreover, any sequence of solutions in $\mathcal{M}^{P}\left(O_{a}, O_{c}\right)$ converging to the boundary lies eventually in the range of the gluing map. 
Proof. The proof of the first two cases is analogous to the case of flow lines analyzed in Section 4, therefore we concentrate on the case of the gluing map (96).

Consider the moduli space

$$
\mathcal{M}_{\sigma=1}^{P}\left(O_{a}, O_{c}\right)=\mathcal{M}^{P}\left(O_{a}, O_{c}\right) \cap\{\sigma=1\},
$$

with the metric $\gamma_{1}=g_{t} \# \tilde{g}_{t}$, as in (81). We define the metric $\gamma_{1}(R)=g_{t} \#{ }_{R} \tilde{g}_{t}$, for sufficiently large $R \geq R_{0}$, as

$$
\gamma_{1}(R)=\left\{\begin{array}{lr}
g_{0}+d t^{2} & t<-2-R \\
g_{t+2}+d t^{2} & t \in[-2-R,-1-R] \\
g_{1}+d t^{2} & t \in[-1-R, 1+R] \\
\tilde{g}_{2-t}+d t^{2} & t \in[1+R, 2+R] \\
g_{0}+d t^{2} & t>2+R .
\end{array}\right.
$$

Correspondingly, we have metrics $\gamma_{\sigma}(R)$, for $\sigma \in[0,1]$. We consider the moduli spaces $\mathcal{M}^{P}\left(O_{a}, O_{c}\right)$ as before, defined with respect to the metrics $\gamma_{\sigma}(R)$. All the properties of these moduli spaces discussed so far, are independent of the choice of a fixed $R$. However, to describe in detail the appearance of strata of the form (97) in the compactification, and their gluing properties, we need to work with a large $R$. This correspond to stretching a long cylinder $Y \times[-1-R, 1+R]$ with the constant metric $g_{1}+d t^{2}$ inside the manifold $Y \times \mathbb{R}$. The proof is obtained via the following argument.

Proposition 6.11. Consider a solution $x=[\mathbb{A}, \Psi]$ in $\mathcal{M}_{\sigma=1}^{P}\left(O_{a}, O_{c}\right)$ on the manifold $\left(Y \times \mathbb{R}, \gamma_{1}(R)\right)$. For every $\epsilon>0$, there exists $R_{0}$, such that, if $R \geq R_{0}$, the restriction of $x$ to $Y \times[-2,2]$ is sufficiently close to a solution $\left[A_{a^{\prime}}, \psi_{a^{\prime}}\right] \in O_{a^{\prime}}$, for some critical orbit $O_{a^{\prime}}$. Moreover, given a pair of solutions $(x, y)$ in the fibered product $(97)$, for $R$ sufficiently large, we can consider the pre-glued solution obtained by splicing together the solutions $x$ and $y$ cut off by functions $\rho_{-}$and $\rho_{+}$supported in $Y \times(-\infty,-1]$ and $Y \times[1, \infty)$, respectively. For $R$ sufficiently large, these approximate solutions can be deformed to actual solutions. Thus, we obtain the gluing map (96) with the desired properties.

Proof of Proposition 6.11. The proof of the first statement follows closely the argument of Proposition 8 of [30]. The gluing argument again follows very closely the argument illustrated in Section 4:2, Theorem 4.9 in the case of the moduli spaces of flow lines. Again, the key feature is the fact that the cokernels Coker $\left(\mathcal{L}_{x}\right)$ and Coker $\left(\mathcal{L}_{y}\right)$ vanish, hence the gluing argument presents no surprise with respect to the case of gluing flow lines. 
All the remaining statements of Theorem 6.10 are proved by the same techniques developed in Section 4.

The main problem now is to extend these gluing theorems to the case of strata involving a contribution from the singular moduli space $\mathcal{M}\left(\theta_{0}, \theta_{1}\right)$. The strategy we develop in the rest of Section 6.1 and in Section 6.2 is to identify precisely the contribution of these singular components (91) to the compactification of the moduli spaces $\mathcal{M}\left(\theta_{0}, O_{a^{\prime}}\right)^{*}$ and to the analogous case for $\mathcal{M}^{P}\left(O_{a}, O_{b}\right)^{*}$. The main technique we employ is the obstruction bundle for the gluing construction and a direct analysis of the zero set of the canonical obstruction section. We aim at showing that all the components of the actual boundary in the compactification $\mathcal{M}\left(O_{a}, O_{a^{\prime}}\right)^{*}$ behave according to the picture described in Section 4.3, hence the arguments of the easy case of Theorem 6.1 involving the properties of the compactifications moduli spaces $\mathcal{M}\left(O_{a}, O_{a^{\prime}}\right)^{*}$ and $\mathcal{M}^{P}\left(O_{a}, O_{b}\right)^{*}$ can be extended to the case of different chambers as well.

Remark 6.12. Recall that, in the proof of Theorem 6.1, we need only consider the case of two sufficiently close metrics and perturbations $\left(g_{0}, \nu_{0}\right)$ and $\left(g_{1}, \nu_{1}\right)$ in two different chambers. Thus, we can assume that the path $\left(g_{t}, \nu_{t}\right)$ satisfies $S F\left(\partial_{\nu_{t}}^{g_{t}}\right)=-2$ and the path $\left(\tilde{g}_{t}, \tilde{\nu}_{t}\right)$ satisfies $S F\left(\partial_{\tilde{\nu}_{t}}^{\tilde{g}_{t}}\right)=+2$. Thus, the compactified moduli spaces $\mathcal{M}\left(O_{a^{\prime}}, O_{a}\right)^{*}$ used in the definition of the map $J$ and in the proof of $D J-J D=0$ all satisfy the properties of Section 4.2 and 4.3 , by simply rephrasing the same arguments. The only problem then remains in the analysis of the moduli spaces needed in the definition of $I$, in the proof of $I D-D I=0$ and in the construction of the chain homotopy.

\subsection{Obstruction bundle and gluing theorems.}

The obstruction bundle technique we discuss in this Section will both complete the proof of the unobstructed gluing theorems, as stated in Proposition 4.21 , and identify the precise boundary structure in the gluing with obstructions.

Let $\mathcal{U}\left(O_{a}, O_{b}\right)$ be the image of $\mathcal{M}\left(O_{a}, O_{c}\right) \times{ }_{O_{c}} \mathcal{M}\left(O_{c}, O_{b}\right)$ under the pregluing map $\#^{0}$, for a large gluing parameter $T>>0$. In the case of a translation invariant metric, analyzed in Section 4, the statement of the gluing theorem 4.9 is equivalent to the statement that the subset

$$
\mathcal{U}\left(O_{a}, O_{b}\right) \subset \mathcal{B}_{k, \delta}^{0}\left(O_{a}, O_{b}\right)
$$


can be deformed to $\mathcal{M}\left(O_{a}, O_{b}\right)$, whenever $\mathcal{U}\left(O_{a}, O_{b}\right)$ is obtained by pasting together solutions along $O_{b}$ with $\mu\left(O_{a}\right)>\mu\left(O_{c}\right)>\mu\left(O_{b}\right)$. It is this equivalent statement which we are going to verify in the proof of Proposition 4.21 given in this Section, following Lemma 6.14.

However, if we consider the moduli spaces $\mathcal{M}\left(O_{a}, O_{a^{\prime}}\right)$ with the metric $\tilde{g}_{t}$ that varies along the cylinder, there might be topological obstructions that make it impossible to deform the set of pre-glued trajectories to actual solutions of (18) and (19). In other words, given a point $\left[x \#_{T}^{0} y\right] \in \mathcal{U}\left(O_{a}, O_{a^{\prime}}\right)$ there might be an obstruction to pushing that point onto $\mathcal{M}\left(O_{a}, O_{a^{\prime}}\right)$. Typically obstructions may arise either because of the presence of non-trivial cokernels of the linearizations $\mathcal{L}_{x}$ and $\mathcal{L}_{y}$, or because Sard's theorem is not available. In the problem we are considering the obstruction originates in the presence of the non-trivial cokernel of Lemma 6.8. Similar topological obstructions to gluing solutions arise in other gauge theory problems [59], [60]. Following Taubes' construction of the obstruction bundle, it is possible to describe the obstruction completely in terms of the vanishing of a canonical section.

We maintain the notation (53) as in the proof of the gluing theorem: for an element $x \#_{T}^{0} y$, with $x=\left(\mathbb{A}_{1}, \Psi_{1}\right)$ and $y=\left(\mathbb{A}_{2}, \Psi_{2}\right)$, we write

$$
f\left(x \#_{T}^{0} y\right)=\left\{\begin{array}{l}
D_{\mathbb{A}_{1} \#_{T}^{0} \mathbb{A}_{2}}\left(\Psi_{1} \#_{T}^{0} \Psi_{2}\right)+\rho\left(\Psi_{1} \#_{T}^{0} \Psi_{2}\right) \\
F_{\mathbb{A}_{1} \#_{T}^{0} \mathbb{A}_{2}}^{+}-\left(\Psi_{1} \#_{T}^{0} \Psi_{2}\right) \cdot \overline{\left(\Psi_{1} \#_{T}^{0} \Psi_{2}\right)}-i \mu-P_{\left(\mathbb{A}_{1} \#_{T}^{0} \mathbb{A}_{2}, \Psi_{1} \#_{T}^{0} \Psi_{2}\right)} \\
G_{\Gamma_{a a^{\prime}}}^{*}\left(\mathbb{A}_{1} \#_{T}^{0} \mathbb{A}_{2}, \Psi_{1} \#_{T}^{0} \Psi_{2}\right),
\end{array}\right.
$$

for a fixed choice of a slice $\mathcal{S}_{\Gamma_{a a^{\prime}}}$ in $\mathcal{A}\left(O_{a}, O_{a^{\prime}}\right)$, such that $x \#_{T}^{0} y$ is in a ball of radius $r$ in $\mathcal{A}\left(O_{a}, O_{a^{\prime}}\right)$ centered at $\Gamma_{a a^{\prime}}$. If the pre-glued element $x \#_{T}^{0} y$ can be deformed to an actual solution, then there exists a small $(\alpha, \Phi) \in$ $\operatorname{Ker}\left(\mathcal{L}_{x \#_{T}^{0} y}\right)^{\perp}$ such that we have

$$
\left[x \#_{T}^{0} y+(\alpha, \Phi)\right] \in \mathcal{M}\left(O_{a}, O_{a^{\prime}}\right),
$$

that is, the following equation is satisfied

$$
f\left(x \#_{T}^{0} y\right)+\mathcal{L}_{x \#_{T}^{0} y}(\alpha, \Phi)+\mathcal{N}_{x \#_{T}^{0} y}(\alpha, \Phi)=0 .
$$

Here $\mathcal{N}$ denotes the nonlinear term in the equation, as in Proposition 4.21.

$$
\mathcal{N}_{x \#_{T}^{0} y}(\alpha, \Phi)=\left(\Phi \cdot \bar{\Phi}+\mathcal{N} P_{x \#_{T}^{0} y}(\alpha, \Phi), \alpha \cdot \Phi\right),
$$

where the perturbation $P$ of equation (88) is written as sum of a linear and a non-linear term, $P=\mathcal{D} P+\mathcal{N} P$. 
The presence of a non-trival approximate kernel of $\mathcal{L}_{x \#_{T}^{0} y}^{*}$ can generate obstructions to solving equation (99) for $(\alpha, \Phi)$. In fact, the hypothesis that $\mathcal{L}_{x \#_{T}^{0} y}$ has a trivial approximate cokernel is essential in the proof of the gluing theorem (see Lemma 4.13 and Proposition 4.19): the same argument cannot be extended to a case with a non-trivial cokernel.

We follow [60] and introduce open sets $\mathcal{U}(\mu)$ of elements $\left[x \#_{T}^{0} y\right] \in$ $\mathcal{U}\left(O_{a}, O_{a^{\prime}}\right)$ such that $\mu>0$ is not an eigenvalue of the operator $\mathcal{L}_{x \#_{T}^{0} y} \mathcal{L}_{x \#_{T}^{*} y}^{*}$ acting on $L_{0, \delta(T)}^{2}$ connections and sections. There are projection maps $\Pi\left(\mu, x \#_{T}^{0} y\right)$ onto the span of the eigenvectors of $\mathcal{L}_{x \#_{T}^{0} y} \mathcal{L}_{x \#_{T}^{0} y}$ with eigenvalue smaller than $\mu$. These are smooth maps of $\left[x \#_{T}^{0} y\right] \in \mathcal{U}(\mu)$.

We have the following result, which gives the necessary eigenvalue splitting for the operators $\mathcal{L}_{x \#_{T}^{0} y} \mathcal{L}_{x \#_{T}^{0} y}$ and $\mathcal{L}_{x \#_{T}^{*} y}^{*} \mathcal{L}_{x \#_{T}^{0} y}$.

Lemma 6.13. There exists a $\mu_{0}=\mu\left(T_{0}\right)>0$, such that, for all $T \geq T_{0}$, all the small eigenvalues $\mu_{T}$ of $\mathcal{L}_{x \#_{T}^{0} y}^{*} \mathcal{L}_{x \#_{T}^{0} y}\left(\right.$ or $\left.\mathcal{L}_{x \#_{T}^{0} y} \mathcal{L}_{x \#_{T}^{0} y}^{*}\right)$, satisfy $\mu_{T}<\mu_{0}$ and $\mu_{T} \rightarrow 0$, and all the other eigenvalues are bounded below by $\mu_{0}$.

Proof. Recall that, from Lemma 4.12 we know that the number of independent eigenvectors of $\mathcal{L}_{x \#_{T}^{0} y}^{*} \mathcal{L}_{x \#_{T}^{0} y}$ or $\mathcal{L}_{x \#_{T}^{0} y} \mathcal{L}_{x \#_{T}^{0} y}^{*}$ with small eigenvalue $\mu_{T} \rightarrow 0$ is at most

$$
\operatorname{dim} \operatorname{Ker}\left(\mathcal{L}_{x}\right)+\operatorname{dim} \operatorname{Ker}\left(\mathcal{L}_{y}\right)
$$

or at most

$$
\operatorname{dim} \operatorname{Coker}\left(\mathcal{L}_{x}\right)+\operatorname{dim} \operatorname{Coker}\left(\mathcal{L}_{y}\right),
$$

respectively. Moreover, by Lemma 4.15, we know that the spans of the eigenvectors of the small eigenvalues have exactly these dimensions, cf. Proposition 4.17. So there exists a $\mu_{0}$ such that, for $T \geq T_{0}$, there is exactly this number of independent eigenvectors with eigenvalues $\mu_{T}<\mu_{0}$ and $\mu_{T} \rightarrow 0$. In fact, we also know more precise estimates on the rate of decay of these small eigenvalues, as specified in Lemma 4.15.

It remains to be shown that such $\mu_{0}$ can be chosen so that all the remaining eigenvalues are bounded below by $\mu_{0}$. In other words, we have to show that we cannot have other sequences of eigenvalues $\mu_{T}(k)$ satisfying $\mu_{T}(k) \rightarrow \mu_{k}>0$, as $T \rightarrow \infty$, but with the limits $\mu_{k} \rightarrow 0$ as $k \rightarrow \infty$. This follows from Lemma 4.13. In fact, we have proved that the operator $\mathcal{L}_{x \#_{T}^{0} y}^{*} \mathcal{L}_{x \#_{T}^{0} y}$ (or $\mathcal{L}_{x \#_{T}^{0} y} \mathcal{L}_{x \#_{T}^{0} y}^{*}$ ) is uniformly invertible on the orthogonal complement of the small eigenvalues eigenspace. Notice that Lemma 4.15 
and Lemma 4.13 give norms for the operator $\mathcal{L}_{x \#_{T}^{0} y}$ (and by similar arguments for the operator $\mathcal{L}_{x \#_{T}^{0} y}^{*}$ ) which are uniformly bounded in $T$, when the operators are considered as acting between Sobolev spaces with the same $\delta$ weight on source and target space (or with the same rescaled weight $\delta(T)$ on both source and target space). In other words we have

$$
\left\|\mathcal{L}_{x \#_{T}^{0} y}^{*} \mathcal{L}_{x \#_{T}^{0} y} \xi\right\|_{L_{1, \delta}^{2}} \geq C\|\xi\|_{L_{1, \delta}^{2}}
$$

for

$$
\xi \in\left(F_{\#_{T}}\left(\operatorname{Ker}\left(\mathcal{L}_{x}\right) \times \operatorname{Ker}\left(\mathcal{L}_{y}\right)\right)\right)^{\perp}
$$

inside

$$
\mathcal{T}_{1, \delta}\left(\left(e_{a}^{+}\right)^{-1}\left(x_{a}\right)\right) \subset \mathcal{T}_{1, \delta}\left(\mathcal{S}_{\Gamma_{a a^{\prime}}}\right)
$$

and

$$
\left\|\mathcal{L}_{x \#_{T}^{0} y} \mathcal{L}_{x \#_{T}^{0} y}^{*} \xi\right\|_{L_{0, \delta}^{2}} \geq C\|\xi\|_{L_{0, \delta}^{2}}
$$

for

$$
\xi \in\left(F_{\# T}\left(\operatorname{Coker}\left(\mathcal{L}_{x}\right) \times \operatorname{Coker}\left(\mathcal{L}_{y}\right)\right)\right)^{\perp}
$$

inside $\mathcal{T}_{0, \delta}\left(\mathcal{S}_{\Gamma_{a a^{\prime}}}\right)$. With this choices of norms, the constants are all independent of $T$. Here $x_{a} \in O_{a}$ is the asymptotic value as $t \rightarrow-\infty$ of the solution $x \in \mathcal{M}\left(O_{a}, O_{a^{\prime}}\right)$, and $e_{a}^{+}: \mathcal{S}_{\Gamma_{a a^{\prime}}} \rightarrow O_{a}$ is the endpoint map, for the fixed choice of slices.

It is important to notice that the difference between using the rescaled $\delta(T)$-norms or the original $\delta$-norms in the previous Lemma is reflected in the different norm bound on the gluing map $\tilde{F}_{\#_{T}}$, as explained in the proof of Theorem 4.9 .

The kind of analysis of the behavior of small eigenvalues described in Lemma 6.13 has been used in similar context in [59], [60], with a fuller account in [49], and in [10]. A similar analysis has also been used, more recently, in [11], [22].

By Lemma 6.13, we now know that there exist $T_{0}>>0$ and $\mu\left(T_{0}\right)>$ 0 , such that, for $T>T_{0}$ and $0<\mu<\mu\left(T_{0}\right)$, we can identify the projection map $\Pi\left(\mu, x \#_{T}^{0} y\right)$ with projection on the approximate cokernel $\operatorname{ApprCoker}\left(\mathcal{L}_{x \#_{T}^{0} y}\right)$, as defined in Definition 4.16.

Consider a compact set $K \subset \mathcal{U}\left(O_{a}, O_{a^{\prime}}\right)$. Suppose it can be covered with one open set $\mathcal{U}(\mu)$. If not, we can paste together the projections corresponding to the finitely many open sets that cover $K$, using a suitable partition of unity as in [60], pg. 193 and slightly modifying the argument that follows in [60]. 
Since the element $(\alpha, \Phi)$ is in $\operatorname{Ker}\left(\mathcal{L}_{x \#_{T}^{0} y}\right)^{\perp}$, we have

$$
(\alpha, \Phi)=\mathcal{L}_{x \#_{T}^{0} y}^{*}(\beta, \xi) .
$$

Suppose that $\left[x \#_{T}^{0} y+(\alpha, \Phi)\right]$ is a solution, for a non-trivial element $(\alpha, \Phi)$. For $\mu$ small enough, we can assume that $(\beta, \xi)$ satisfies

$$
(\beta, \xi) \in \operatorname{Ker}\left(\Pi\left(\mu, x \#_{T}^{0} y\right)\right) .
$$

Then $(\beta, \xi)$ solves the equations

$$
\begin{aligned}
& \text { (100) } \mathcal{L}_{x \#_{T}^{0} y} \mathcal{L}_{x \#_{T}^{0} y}^{*}(\beta, \xi)
\end{aligned}
$$

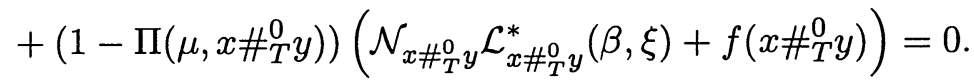

and

$$
\Pi\left(\mu, x \#_{T}^{0} y\right)\left(\mathcal{N}_{x \#_{T}^{0} y} \mathcal{L}_{x \#_{T}^{0} y}^{*}(\beta, \xi)+f\left(x \#_{T}^{0} y\right)\right)=0 .
$$

Lemma 6.14 below ensures that it is always possible to find a small solution of equation (100), hence the problem of deforming an approximate solution to an actual solution depends on whether equation (101) can also be solved. The latter has a geometric interpretation as the section of an obstruction bundle, as described in the following (see [16] Section 4, [59] Section 3 and 5, [60] Section 6).

Lemma 6.14. There is an $\epsilon>0$ and a constant $C>0$ such that, for any $\mu$ with $C \epsilon<\mu<\mu_{0}$, with $\mu_{0}$ the least eigenvalue of $H_{x \#_{T}^{0} y}^{0}$ on the complement of ApprCoker $\left(\mathcal{L}_{x \#_{T}^{0} y}\right)$, the equation (100) has a unique solution

$$
(\beta, \xi) \in \operatorname{Ker}\left(\Pi\left(\mu, x \#_{T}^{0} y\right)\right)
$$

with $\|(\beta, \xi)\|_{L_{2, \delta}^{2}} \leq \epsilon$, provided the error term $\left\|f\left(x \#_{T}^{0} y\right)\right\|$ satisfies an estimate

$$
\left\|f\left(x \#_{T}^{0} y\right)\right\| \leq \frac{\mu}{2 \epsilon} .
$$

Proof. For $\mu>0$ and any $(\mathbb{A}, \Psi)$, the operator

$$
H_{(\mathbb{A}, \Psi)}^{0}=\mathcal{L}_{(\mathbb{A}, \Psi)} \mathcal{L}_{(\mathbb{A}, \Psi)}^{*}
$$


has a bounded inverse, when restricted to the image of $(1-\Pi(\mu,(\mathbb{A}, \Psi))$, in the space of $L_{\delta}^{2}$ connections and sections, as discussed in Lemma 4.13.

Thus, the equation (100) can be rephrased as a fixed point problem

$$
(\beta, \xi)=-H_{x \#_{T}^{0} y}^{0}{ }^{-1}\left(1-\Pi\left(\mu, x \#_{T}^{0} y\right)\right)\left(\mathcal{N}_{x \#_{T}^{0} y} \mathcal{L}_{x \#_{T}^{0} y}^{*}(\beta, \xi)+f\left(x \#_{T}^{0} y\right)\right)
$$

We need to prove that the right hand side is a contraction. For all $(\beta, \xi)$ and $\left(\beta^{\prime}, \xi^{\prime}\right)$, we have an estimate

$$
\begin{aligned}
& \left\|H_{x \#_{T}^{0} y}{ }^{-1}\left(1-\Pi\left(\mu, x \#_{T}^{0} y\right)\right)\left(\mathcal{N} \mathcal{L}^{*}(\beta, \xi)-\mathcal{N} \mathcal{L}^{*}\left(\beta^{\prime}, \xi^{\prime}\right)\right)\right\|_{L_{\delta}^{2}} \\
& \leq \frac{1}{\mu}\left\|(1-\Pi)\left(\mathcal{N} \mathcal{L}^{*}(\beta, \xi)-\mathcal{N} \mathcal{L}^{*}\left(\beta^{\prime}, \xi^{\prime}\right)\right)\right\|_{L_{\delta}^{2}} \\
& \leq \frac{1}{\mu}\left\|\mathcal{N} \mathcal{L}^{*}(\beta, \xi)-\mathcal{N} \mathcal{L}^{*}\left(\beta^{\prime}, \xi^{\prime}\right)\right\|_{L_{\delta}^{2} .}
\end{aligned}
$$

The quadratic form $(\phi \cdot \bar{\phi}, \alpha \cdot \phi)$ satisfies an estimate of the form

$$
\|N(x)-N(y)\| \leq C(\|x\|+\|y\|)\|x-y\|
$$

as required for the contraction method 4.20 .

The perturbation term also satisfies a similar estimate for large enough $T$ because of the assumptions on the perturbation space $\mathcal{P}$. Thus we can improve the last estimate to get

$$
\begin{aligned}
& \frac{1}{\mu}\left\|\mathcal{N} \mathcal{L}^{*}(\beta, \xi)-\mathcal{N} \mathcal{L}^{*}\left(\beta^{\prime}, \xi^{\prime}\right)\right\|_{L_{\delta}^{2}} \\
& \leq \frac{\tilde{C}}{\mu}\left(\left\|\mathcal{L}^{*}(\beta, \xi)\right\|+\left\|\mathcal{L}^{*}\left(\beta^{\prime}, \xi^{\prime}\right)\right\|\right)\left\|\mathcal{L}^{*}\left((\beta, \xi)-\left(\beta^{\prime}, \xi^{\prime}\right)\right)\right\|_{L_{1, \delta}^{2}} \\
& \leq \frac{C}{\mu}\left(\|(\beta, \xi)\|+\left\|\left(\beta^{\prime}, \xi^{\prime}\right)\right\|\right)\left\|(\beta, \xi)-\left(\beta^{\prime}, \xi^{\prime}\right)\right\|_{L_{2, \delta}^{2}} \cdot
\end{aligned}
$$

Thus, we have

$$
\begin{aligned}
& \left\|H_{x \#_{T}^{0} y}^{0}{ }^{-1}\left(1-\Pi\left(\mu, x \#_{T}^{0} y\right)\right)\left(\mathcal{N}_{x \#_{T}^{0} y} \mathcal{L}_{x \#_{T}^{0} y}^{*}(\beta, \xi)+f\left(x \#_{T}^{0} y\right)\right)\right\| \\
& \leq \frac{C}{\mu}\|(\beta, \xi)\|+\frac{1}{\mu}\left\|f\left(x \#_{T}^{0} y\right)\right\| .
\end{aligned}
$$

Suppose given $\epsilon$ and $\mu$ satisfying

$$
\max \{2 C \epsilon, \epsilon\}<\mu<\mu_{0},
$$


and assume the the error term also satisfies

$$
\left\|f\left(x \#_{T}^{0} y\right)\right\| \leq \frac{\mu}{2 \epsilon} .
$$

Then the map is a contraction on the ball $\|(\beta, \xi)\|_{L_{\delta}^{2}} \leq \epsilon$.

Remark 6.15. Lemma 6.14 relies on the identification of the image of $1-$ $\Pi\left(\mu, x \#_{T}^{0} y\right)$, that is, the complement of the small eigenvalues eigenspace for the Laplacian $H_{x \#_{T}^{0} y}^{0}$, with the space

$$
F_{\# T}\left(\operatorname{Coker}\left(\mathcal{L}_{x}\right) \times \operatorname{Coker}\left(\mathcal{L}_{y}\right)\right) .
$$

This identification is obtained from Lemma 4.13 together with Remark 4.14. In particular, recall that, by Lemma 4.13 and Remark 4.14, the isomorphism

$$
F_{\# T}: \text { Coker } \mathcal{L}_{x} \times \operatorname{Coker} \mathcal{L}_{y} \cong \text { Image }\left(1-\Pi\left(\mu, x \#_{T}^{0} y\right)\right)
$$

has norm bounded uniformly in $T$ if we use weighted norms with rescaled weight $\delta(T)$ on the target space and the $\delta$-norms on the source spaces. It has norm bounded by $C e^{-\delta T}$ if the target space is also endowed with the $\delta$-norm.

Remark 6.16. In the case of the gluing theorem for flow lines, with approximate solutions $x \#_{T}^{0} y$ in $\mathcal{U}\left(O_{a}, O_{c}\right)$, obtained from elements $x \in \hat{\mathcal{M}}\left(O_{a}, O_{b}\right)$ and $y \in \hat{\mathcal{M}}\left(O_{b}, O_{c}\right)$, it is possible to choose the constant $\epsilon$ in Lemma 6.14 independent of $T \geq T_{0}$. In fact, in this case it is possible to choose $\mu \leq \mu_{0}$, the least eigenvalue of $H_{x \#_{T}^{0} y}^{0}$, which in this case is independent of $T$, cf. the spectral decomposition of Lemma 6.13. The constant $C$ is also independent of $T \geq T_{0}$, cf. Lemma 6.13. Thus, in this case, the projection $\Pi\left(\mu, x \#_{T}^{0} y\right)$ is trivial, hence the gluing result reduces to the following argument that proves Proposition 4.21.

A proof of Proposition 4.21 follows from Lemma 6.14 in the case the cylindrical metric on $Y \times \mathbb{R}$, and under the assumptions that Coker $\left(\mathcal{L}_{x}\right)=0$ and $\operatorname{Coker}\left(\mathcal{L}_{y}\right)=0$.

Proof of Proposition 4.21. Let us consider the space $\mathcal{T}_{1, \delta}\left(\mathcal{S}_{\Gamma_{a c}}\right)$, endowed with the $L_{1, \delta}^{2}-$ norm. The initial condition of Remark 4.20 is provided by the exponential decay. In fact, we have

$$
\begin{aligned}
& \left\|f\left(\mathbb{A}_{1} \#_{T}^{0} \mathbb{A}_{2}, \Psi_{1} \#_{T}^{0} \Psi_{2}\right)\right\|_{L_{1, \delta}^{2}} \\
& \leq\left\|F_{\# T}\right\| \cdot\left(\left\|\left.\left(\alpha_{1}, \phi_{1}\right)\right|_{Y \times[-1+T, T]}\right\|_{L_{1, \delta}^{2}}+\left\|\left.\left(\alpha_{2}, \phi_{2}\right)\right|_{Y \times[-T,-T+1]}\right\|_{L_{1, \delta}^{2}}\right) .
\end{aligned}
$$


The operator norm $\left\|F_{\#_{T}}\right\|$, for $F_{\#_{T}}$ acting on the $L_{1, \delta}^{2}$ spaces, is bounded by $C e^{-\delta T}$, as in (45). The terms

$$
\left\|\left.\left(\alpha_{1}, \phi_{1}\right)\right|_{Y \times[-1+T, T]}\right\|_{L_{1, \delta}^{2}} \text { and }\left\|\left.\left(\alpha_{2}, \phi_{2}\right)\right|_{Y \times[-T,-T+1]}\right\|_{L_{1, \delta}^{2}}
$$

are bounded by a constant, uniformly in $T \geq T_{0}$, because of the exponential decay of $\left(A_{1}(t), \psi_{1}(t)\right)$ and $\left(A_{2}(t), \psi_{2}(t)\right)$ to the endpoints.

Thus, for all $T \geq T_{0}$, we have obtained an estimate

$$
\left\|f\left(\mathbb{A}_{1} \#_{T}^{0} \mathbb{A}_{2}, \Psi_{1} \#_{T}^{0} \Psi_{2}\right)\right\|_{1, \delta} \leq C e^{-\delta T} .
$$

This result can be adapted to prove all the unobstructed gluing results, such as the ones stated in Theorems 6.9 and 6.10. Now we turn to the more interesting case with obstructions.

Using the result of Lemma 6.14, we can construct the obstruction bundle and the canonical section, in the case with non-trivial approximate cokernel (i.e., non-trivial small eigenvalue eigenspace of $H_{x \#_{T}^{0} y}^{0}$ ).

Proposition 6.17. There is a local bundle $\mathcal{E}$ over $\mathcal{U}\left(O_{a}, O_{a^{\prime}}\right)$ and a section $s_{\mu}$ such that the following property holds. A point $x \#_{T}^{0} y$ in $\mathcal{U}\left(O_{a}, O_{a^{\prime}}\right)$ can be deformed to a solution of the flow equations (18) and (19) iff

$$
x \#_{T}^{0} y \in s_{\mu}^{-1}(0) .
$$

Proof. Consider the section

$$
s_{\mu}\left(x \#_{T}^{0} y\right)=\Pi\left(\mu, x \#_{T}^{0} y\right)\left(\mathcal{N}_{x \#_{T}^{0} y} \mathcal{L}_{x \#_{T}^{0} y}^{*}(\beta, \xi)+f\left(x \#_{T}^{0} y\right)\right)
$$

of the vector bundle over $\mathcal{U}\left(O_{a}, O_{a^{\prime}}\right)$ with fiber $\operatorname{ApprCoker}\left(\mathcal{L}_{x \#_{T}^{0} y}\right)$. Here $(\beta, \xi)$ is the unique solution of $(100)$.

Since $(\beta, \xi)$ satisfies $(100)$, we have

$$
\left[x \#_{T}^{0} y+\mathcal{L}_{x \#_{T}^{0} y}^{*}(\beta, \xi)\right] \in \mathcal{M}\left(O_{a}, O_{a^{\prime}}\right)
$$

iff

$$
s_{\mu}\left(x \#_{T}^{0} y\right)=0
$$


In the case of moduli spaces of solutions of the Seiberg-Witten equations on $Y \times \mathbb{R}$ with the changing metric $g_{t}+d t^{2}$, as in the construction of the map $I$ and the subsequent arguments, Proposition 6.17 characterizes which points of $\mathcal{U}\left(O_{a}, O_{a^{\prime}}\right)$ can be deformed to actual solutions. We need a more explicit description of this obstruction bundle in the specific problem at hand, namely in the case of approximate solutions $\mathcal{U}\left(\theta_{0}, O_{a^{\prime}}\right)$, with the pregluing map

$$
\#^{0}: \mathcal{M}\left(\theta_{0}, \theta_{1}\right) \times \hat{\mathcal{M}}\left(\theta_{1}, O_{a^{\prime}}\right) \times\left[T_{0}, \infty\right) \rightarrow \mathcal{U}\left(\theta_{0}, O_{a^{\prime}}\right)
$$

or $\mathcal{U}\left(O_{a}, \theta_{1}\right)$ with the pre-gluing map

$$
\#^{0}: \hat{\mathcal{M}}\left(O_{a}, \theta_{0}\right) \times \mathcal{M}\left(\theta_{0}, \theta_{1}\right) \times\left[T_{0}, \infty\right) \rightarrow \mathcal{U}\left(O_{a}, \theta_{1}\right) .
$$

We first need to derive the equivalent of Remark 6.16. Namely, we need a suitable choice of $\mu$ and $\epsilon$, so that the map in the fixed point problem (102) is a contraction. This choice depends upon estimating the error term $\left\|f\left(x \#_{T}^{0} y\right)\right\|$. We have the following result.

Lemma 6.18. Let $x=[\mathbb{A}, 0]$ be the unique reducible in $\mathcal{M}\left(\theta_{0}, \theta_{1}\right)$, with $\mu\left(\theta_{0}\right)-\mu\left(\theta_{1}\right)=-2$. Consider (right)pre-glued solutions $x \#_{T}^{0} y$ in $\mathcal{U}\left(\theta_{0}, O_{a^{\prime}}\right)$, with $y \in \hat{\mathcal{M}}\left(\theta_{1}, O_{a^{\prime}}\right)$. We have an estimate on the error term $\left\|f\left(x \#_{T}^{0} y\right)\right\|$ of the form

$$
\left\|f\left(x \#_{T}^{0} y\right)\right\|_{L_{1, \delta}^{2}} \leq \tilde{C},
$$

for some constant independent of $T \geq T_{0}$, satisfying $\tilde{C}<\mu_{0}$. Moreover, choose $\tilde{C}<\mu<\mu_{0}$, independent of $T$, with $\tilde{C}$ as above, and with $\mu_{0}$ the lower bound on the spectrum of the Laplacian $H_{x \#_{T}^{0}}^{0}$ acting on the complement of the space of small eigenvalues, as in Lemma 6.13. Then for $\max \{2 C \epsilon, \epsilon\}<$ $\mu$, we obtain that the map of (102) is a contraction, and the fixed point problem has a unique solution as in Lemma 6.14. The case of the other pre-gluing maps is completely analogous.

Proof. Recall that $(\mathbb{A}, 0)$ is the unique solution to $d^{+} \mathbb{A}=\mu$ and $d^{*} \mathbb{A}=0$. Here we have the 4-dimensional $d^{*}$, with $*_{4}$ with respect to the metric $g_{t}+d t^{2}$, and $\mu$ of the form $\mu=d \nu_{t}+*_{3} d \nu_{t} \wedge d t$, with $*_{3}$ with respect to the metric $g_{t}$. We have

$$
\begin{gathered}
\left\|f\left(x \#_{T}^{0} y\right)\right\| \leq C\left\|F_{\# T}\right\| \cdot \\
\left(\left\|\left(\alpha_{1}, \phi_{1}\right)\right\|_{L_{1, \delta}^{2}(Y \times[-T-1,-T+1])}+\left\|\mathbb{A}-\mathbb{A}_{1}\right\|_{L_{1, \delta}^{2}(Y \times[T-1, T+1])}\right) .
\end{gathered}
$$


Here $\mathbb{A}_{1}$ is the solution of $d^{+} \mathbb{A}=\mu_{1}$ and $d^{*} \mathbb{A}=0$, with $\mu_{1}=d \nu_{1}+*_{3} d \nu_{1} \wedge d t$. Here we have the 4-dimensional $d^{*}$, with $*_{4}$ with respect to the metric $g_{1}+d t^{2}$ and $*_{3}$ with respect to the metric $g_{1}$. In the rest of the proof we write $d^{*} g_{t}$ and $d^{*} g_{1}$ and $*_{g_{t}}$ and $*_{g_{1}}$ for the 4-dimensional $*$-operators with respect to the two different metrics. (We hope this will not cause confusion with our previous notation $*_{4}$ and $*_{3}$.) The norm $\left\|F_{\#_{T}}\right\|$ is exponentially small $\left\|F_{\# T}\right\| \leq C e^{-\delta T}$. The term $\left\|\left(\alpha_{1}, \phi_{1}\right)\right\|_{L_{1, \delta}^{2}(Y \times[-T-1,-T+1])}$ is bounded by a constant because of the exponential decay of $\left(\alpha_{1}, \phi_{1}\right)$, where we write $y=\lambda_{1}\left(A_{1}+\nu_{1}, 0\right)+\left(\alpha_{1}, \phi_{1}\right)$, as $t \rightarrow-\infty$. The estimate for the remaining term is obtained as follows. We write

$$
\mathbb{A}-\mathbb{A}_{1}=d^{* g_{t}}\left(\mu_{t}-\mu_{1}\right)+\left(d^{* g_{t}}-d^{* g_{1}}\right) \mu_{1} .
$$

Thus, we have

$$
\begin{aligned}
\left\|\mathbb{A}-\mathbb{A}_{1}\right\|_{L_{1}^{2}(Y \times[T-1, T+1])} \leq & \left\|d^{* g_{t}}\left(\mu_{t}-\mu_{1}\right)\right\|_{L_{1}^{2}(Y \times[T-1, T+1])} \\
& +\left\|\left(d^{* g_{t}}-d^{* g_{1}}\right) \mu_{1}\right\|_{L_{1}^{2}(Y \times[T-1, T+1])} .
\end{aligned}
$$

The first term in the right hand side goes to zero for $T$ sufficiently large, because the path $\nu_{t}$ ends at $\nu_{1}$. Upon writing

$$
d^{* g_{t}}-d^{*_{g_{1}}}=*_{g_{t}} \circ d \circ\left(*_{g_{t}}-*_{g_{1}}\right)+\left(*_{g_{t}}-*_{g_{1}}\right) \circ d \circ *_{g_{1}}
$$

we estimate the second term by a constant times the norm $\left\|*_{g_{t}}-*_{g_{1}}\right\|$, using the fact that $d$ is invertible on $\operatorname{Ker}\left(d^{*}\right)$, since $Y$ is a rational homology sphere. The difference of the *-operators is controlled by the difference $\mid \sqrt{\left|g_{t}\right|}-$ $\sqrt{\left|g_{1}\right|} \mid$, with $|g|$ the absolute value of the determinant of the metric. For $\left(g_{0}, \nu_{0}\right)$ and $\left(g_{1}, \nu_{1}\right)$ two sufficiently close points in two different chambers, the path $\left(g_{t}, \nu_{t}\right)$ can be chosen so that this term is small enough, so that we obtain an estimate

$$
\left\|F_{\# T}\right\| \cdot\left\|\mathbb{A}-\mathbb{A}_{1}\right\|_{L_{1, \delta}^{2}(Y \times[T-1, T+1])} \leq \tilde{C},
$$

with $\tilde{C}<\mu_{0}$, after combining the resulting estimate

$$
\left\|\mathbb{A}-\mathbb{A}_{1}\right\|_{L_{1}^{2}(Y \times[T-1, T+1])} \leq C_{1},
$$

which gives

$$
\left\|\mathbb{A}-\mathbb{A}_{1}\right\|_{L_{1, \delta}^{2}(Y \times[-T-1,-T+1])} \leq C_{1} e^{\delta T}
$$

with the estimate $\left\|F_{\#_{T}}\right\| \leq C e^{-\delta T}$. 
This gives the required estimate for the error term. We then have the estimate

$$
\begin{aligned}
& \left\|H_{x \#_{T}^{0} y}^{0-1}\left(1-\Pi\left(\mu, x \#_{T}^{0} y\right)\right)\left(\mathcal{N}_{x \#_{T}^{0} y} \mathcal{L}_{x \#_{T}^{0} y}^{*}(\beta, \xi)+f\left(x \#_{T}^{0} y\right)\right)\right\| \\
& \leq \frac{C}{\mu}\|(\beta, \xi)\|+\frac{1}{\mu}\left\|f\left(x \#_{T}^{0} y\right)\right\| \\
& \leq \frac{C}{\mu} \epsilon+\frac{\tilde{C}}{\mu}
\end{aligned}
$$

With the choice of $\mu$ and $\epsilon$ as indicated, the map is a contraction. The case of (right)pre-gluing maps is completely analogous.

For $T \geq T_{0}$ sufficiently large, the projection $\Pi\left(\mu, x \#_{T}^{0} y\right)$ is identified with the projection on the approximate cokernel

$$
\operatorname{ApprCoker}\left(\mathcal{L}_{x \#_{T}^{0} y}\right) \cong \operatorname{Coker}\left(\mathcal{L}_{y}\right) \cong \mathbb{C}
$$

Thus, the construction of the obstruction bundle in [59] can be rephrased as in Theorem 4.53 of [16]. Namely, we obtain the following description of the obstruction.

Theorem 6.19. Consider the space $\mathcal{U}\left(\theta_{0}, O_{a^{\prime}}\right)$, obtained by gluing $x=$ $(\mathbb{A}, 0)$ in $\mathcal{M}\left(\theta_{0}, \theta_{1}\right)$ with $y \in \mathcal{M}\left(\theta_{1}, O_{a^{\prime}}\right)$. There exists a bundle $\tilde{\mathcal{E}}$ over $\mathcal{M}\left(\theta_{1}, O_{a^{\prime}}\right) / U(1)$ with fiber

$$
\operatorname{Coker}\left(\mathcal{L}_{x}\right)=\mathbb{C}
$$

with the following property. The set of pre-glued elements $x \#_{T}^{0} y$ in $\mathcal{U}\left(\theta_{0}, O_{a^{\prime}}\right)$ that can be deformed to an actual solution in $\mathcal{M}\left(\theta_{0}, O_{a^{\prime}}\right)$ form a codimension 2 submanifold of

$$
\mathcal{M}\left(\theta_{1}, a^{\prime}\right) \cong \mathcal{M}\left(\theta_{1}, O_{a^{\prime}}\right) / U(1)
$$

given by the zero set of a transverse section of $\tilde{\mathcal{E}}$. The case of $\mathcal{U}\left(O_{a}, \theta_{1}\right)$ is analogous.

Proof. As a result of Lemma 6.18, we know that, in the case of $\mathcal{U}\left(\theta_{0}, O_{a^{\prime}}\right)$, the obstruction bundle described in Proposition 6.17 is a bundle $\mathcal{E}$ over $\mathcal{M}\left(\theta_{0}, \theta_{1}\right) \times \mathcal{M}\left(\theta_{1}, O_{a^{\prime}}\right)$ with fiber ApprCoker $\left(\mathcal{L}_{x \#_{T}^{0} y}\right)$. Since we are considering the case with $\mu\left(\theta_{0}\right)-\mu\left(\theta_{1}\right)<0$, according to Lemma 6.6, the moduli 
space $\mathcal{M}\left(\theta_{0}, \theta_{1}\right)$ consists of a unique gauge class $[\mathbb{A}, 0]$. Thus we have a diffeomorphism

$$
\pi: \mathcal{M}\left(\theta_{1}, O_{a^{\prime}}\right) \stackrel{\cong}{\rightrightarrows} \mathcal{U}\left(\theta_{0}, O_{a^{\prime}}\right)
$$

induced by the pre-gluing map. According to Corollary 4.17, since we can restrict our attention to the case $\mu\left(\theta_{0}\right)-\mu\left(\theta_{1}\right)=-2$, we have

$$
\operatorname{ApprCoker}\left(\mathcal{L}_{x \#_{T}^{0} y}\right) \cong \operatorname{Coker}\left(\mathcal{L}_{x}\right) \cong \mathbb{C} \text {. }
$$

Consider the bundle $\tilde{\mathcal{E}}$ over $\mathcal{M}\left(\theta_{1}, O_{a^{\prime}}\right)$ with fiber

$$
\operatorname{Coker}\left(\mathcal{L}_{x}\right) \cong \mathbb{C}
$$

obtained as pullback of the obstruction bundle $\mathcal{E}$ via the diffeomorphism $\pi$ of the base spaces. There is a section $\tilde{s}=\pi^{*} s_{\mu}$ of $\tilde{\mathcal{E}}$ that corresponds to the canonical section $s_{\mu}$ of Proposition 6.17. Recall that there is a free $U(1)$ action over the moduli space $\mathcal{M}\left(\theta_{1}, O_{a^{\prime}}\right)$, hence there is a smooth projection to the quotient

$$
\mathcal{M}\left(\theta_{1}, O_{a^{\prime}}\right) / U(1) \cong \mathcal{M}\left(\theta_{1}, a^{\prime}\right) .
$$

The bundle $\tilde{\mathcal{E}}$ can be regarded as the pullback, under this quotient map, of a bundle $\overline{\mathcal{E}}$ over $\mathcal{M}\left(\theta_{1}, O_{a^{\prime}}\right) / U(1)$, with fiber

$$
\operatorname{Coker}\left(\mathcal{L}_{x}\right) \cong \mathbb{C} \text {. }
$$

The section $\tilde{s}$ is the pullback of a corresponding section $\bar{s}$ of $\overline{\mathcal{E}}$. We are going to proceed as follows. We show that, for a generic choice of the perturbations $\rho$ and $P$ of equations (87) and (88), this section $\bar{s}$ is a generic section of $\overline{\mathcal{E}}$.

Consider the universal pre-glued space

$$
\tilde{\mathcal{U}}\left(\theta_{1}, O_{a^{\prime}}\right)=\left\{\left(\rho, P, x \#_{T}^{0} y\right)\right\}
$$

with $\rho$ a compactly supported form in $\Lambda_{c}^{1}(Y \times \mathbb{R}), P$ a perturbation of $\mathcal{P}$ as in Definition 2.10 (with the modified property (1') specified at the beginning of Section 6.1). The pre-glued element $x \#_{T}^{0} y$ is obtained from $x$ and $y$, solutions respectively of the equations (87), (88) and (20), (21), with the perturbations $\rho$ and $P$. We can extend the bundle $\tilde{\mathcal{E}}$ to a local bundle over $\tilde{\mathcal{U}}\left(\theta_{1}, O_{a^{\prime}}\right)$ with fiber $\operatorname{Coker}\left(\mathcal{L}_{x}\right)$. We can still identify this as the pullback of a local bundle $\overline{\mathcal{E}}$ on the quotient $\tilde{\mathcal{U}}\left(\theta_{1}, O_{a^{\prime}}\right) / U(1)$. There is an induced section $\bar{s}$ of $\overline{\mathcal{E}}$ whose pull-back agrees with the section $\pi^{*} s_{\mu}$ for fixed perturbations $(\rho, P)$. 
We prove that this section $\bar{s}$ over $\tilde{\mathcal{U}}\left(\theta_{1}, O_{a^{\prime}}\right) / U(1)$ is transverse to the zero section, hence the restriction to a generic $(\rho, P)$ gives a generic section over $\mathcal{U}\left(\theta_{1}, O_{a^{\prime}}\right) / U(1)$.

The section $\bar{s}$ is given by

$$
\bar{s}\left(\rho, P, x \#_{T}^{0} y\right)=\Pi_{\mathrm{Coker}\left(\mathcal{L}_{x}\right)}\left(\mathcal{N}_{\left(\rho, P, x \#_{T}^{0} y\right)} \mathcal{L}_{\left(\rho, P, x \#_{T}^{0} y\right)}^{*}(\beta, \xi)+f_{(\rho, P)}\left(x \#_{T}^{0} y\right)\right) .
$$

Suppose given a point $\left(\rho, P, x \#_{T}^{0} y\right)$ in $\bar{s}^{-1}(0)$. Consider a small variation of the perturbation $\rho+\epsilon \eta$. The variation of the term $f_{(\rho+\epsilon \eta, P)}\left(x \#_{T}^{0} y\right)$ is given by

$$
\eta \cdot \Psi_{1} \#_{T}^{0} \Psi_{2}=\eta \cdot \rho_{T}^{+} \Psi_{2}^{-2 T} .
$$

Let $\Phi_{1}$ and $\Phi_{2}$ in $\operatorname{Ker}\left(D_{\mathbb{A}-\rho}\right)$ be the generators of the 2-dimensional space Coker $\left(\mathcal{L}_{x}\right)$. Consider a small open sets $U_{i}$ where $\Phi_{i}$ and $\rho_{T}^{+} \Psi_{2}^{-2 T}$ are non-vanishing, and almost constant. There exist 1-forms $\eta_{i}$ supported in small neighborhoods of the open sets $U_{i}$ such that

$$
\left\langle\Phi_{i}, \eta_{i} \cdot \rho_{T}^{+} \Psi_{2}^{-2 T}\right\rangle
$$

is non-zero on $U_{i}$. Thus, we obtain

$$
\int_{Y \times \mathbb{R}}\left\langle\Phi_{i}, \eta_{i} \cdot \rho_{T}^{+} \Psi_{2}^{-2 T}\right\rangle d v \neq 0 .
$$

Thus, by varying the perturbation $\rho$ alone, it is possible to achieve surjectivity of the linearization of the section $\bar{s}$ onto $\operatorname{Coker}\left(\mathcal{L}_{x}\right)$.

There is a free $U(1)$-action on the space $\mathcal{M}\left(\theta_{1}, O_{a^{\prime}}\right)$, whereas the element $[x]=[\mathbb{A}, 0]$ in $\mathcal{M}\left(\theta_{0}, \theta_{1}\right)$ is fixed by the $U(1)$ action. The section

$$
\tilde{s}=\pi^{*} s_{\mu}: \mathcal{M}\left(\theta_{1}, O_{a^{\prime}}\right) \rightarrow \tilde{\mathcal{E}}
$$

is invariant under the $U(1)$-action, being the pullback of $\bar{s}$. We have seen that, for a generic choice of the perturbation $(\rho, P)$, the section $\bar{s}$ is a generic section. Thus, the approximate solutions in $\mathcal{U}\left(\theta_{0}, O_{a^{\prime}}\right)$ that can be glued to actual solutions in $\mathcal{M}\left(\theta_{0}, O_{a^{\prime}}\right)$ are identified with the co-dimension $2 U(1)$ submanifold

$$
\tilde{s}^{-1}(0) \subset \mathcal{M}\left(\theta_{1}, O_{a^{\prime}}\right) .
$$

In other words, we have then proved that the bundle $\overline{\mathcal{E}}$ over the moduli space $\mathcal{M}\left(\theta_{1}, O_{a^{\prime}}\right) / U(1)$ is a model of the obstruction.

We have the following consequence of Theorem 6.19 and Lemma 6.18. 
Proposition 6.20. For any compact set

$$
K \subset \mathcal{M}\left(\theta_{0}, \theta_{1}\right) \times \tilde{s}^{-1}(0),
$$

with $\tilde{s}^{-1}(0) \subset \mathcal{M}\left(\theta_{1}, O_{a^{\prime}}\right)$ the zeroes of the obstruction section

$$
\tilde{s}^{-1}(0) / U(1)=\bar{s}^{-1}(0) \subset \mathcal{M}\left(\theta_{1}, a^{\prime}\right),
$$

there is an orientation preserving gluing map

$$
\#: K \subset \mathcal{M}\left(\theta_{0}, \theta_{1}\right) \times \tilde{s}^{-1}(0) \rightarrow \mathcal{M}\left(\theta_{0}, O_{a^{\prime}}\right)
$$

that is a smooth embedding. There is a similar gluing map for any compact set

$$
K \subset \tilde{s}^{-1}(0) \times \mathcal{M}\left(\theta_{0}, \theta_{1}\right),
$$

with $\tilde{s}^{-1}(0) \subset \mathcal{M}\left(O_{a}, \theta_{0}\right)$ the zeroes of the obstruction section

$$
\tilde{s}^{-1}(0) / U(1)=\bar{s}^{-1}(0) \subset \mathcal{M}\left(a, \theta_{0}\right) .
$$

Finally, we have the following result on the singular components in the ideal boundary of the moduli spaces $\mathcal{M}\left(O_{a}, O_{a^{\prime}}\right)$.

Theorem 6.21. Assume that $\mu(a)-\mu\left(\theta_{0}\right) \geq 2$ and $\mu\left(\theta_{1}\right)-\mu\left(a^{\prime}\right) \geq 3$, then the contributions of the singular strata

$$
\mathcal{M}\left(\theta_{0}, \theta_{1}\right) \times \mathcal{M}\left(\theta_{1}, O_{a^{\prime}}\right)
$$

and

$$
\mathcal{M}\left(O_{a}, \theta_{0}\right) \times \mathcal{M}\left(\theta_{0}, \theta_{1}\right)
$$

to the actual boundary of the compactified moduli spaces

$$
\mathcal{M}\left(\theta_{0}, O_{a^{\prime}}\right)^{*} \text { and } \mathcal{M}\left(O_{a}, \theta_{1}\right)^{*}
$$

are given by the terms

$$
\mathcal{M}\left(\theta_{0}, \theta_{1}\right) \times \partial^{(1)}\left(\tilde{s}^{-1}(0) \cap \mathcal{M}\left(\theta_{1}, O_{a^{\prime}}\right)\right)
$$

and

$$
\partial^{(1)}\left(\tilde{s}^{-1}(0) \cap \mathcal{M}\left(O_{a}, \theta_{0}\right)\right) \times \mathcal{M}\left(\theta_{0}, \theta_{1}\right),
$$


respectively, where $\tilde{s}$ are the obstruction sections. Thus, the compactification of the moduli spaces $\mathcal{M}\left(O_{a}, O_{a^{\prime}}\right)$ has the structure of a smooth manifold with corners, but in addition to the strata of the form (78), we also have the strata (106) and (107). Thus, we obtain

$$
\begin{aligned}
\partial^{(1)} \mathcal{M}\left(\theta_{0}, O_{a^{\prime}}\right)^{*}= & \bigcup_{\left\{a \mid \mu\left(O_{b^{\prime}}\right)>\mu\left(O_{a^{\prime}}\right)\right\}}\left(\mathcal{M}\left(\theta_{0}, O_{b^{\prime}}\right)^{*} \times{ }_{O_{b^{\prime}}} \hat{\mathcal{M}}\left(O_{b^{\prime}}, O_{a^{\prime}}\right)^{*}\right) \\
& \bigcup_{\left\{b \mid \mu\left(O_{b}\right)<\mu\left(\theta_{0}\right)\right\}}\left(-\hat{\mathcal{M}}\left(\theta_{0}, O_{b}\right)^{*} \times{ }_{O_{b}} \mathcal{M}\left(O_{b}, O_{b^{\prime}}\right)^{*}\right) \\
& \cup \mathcal{M}\left(\theta_{0}, \theta_{1}\right) \times \partial^{(1)}\left(\tilde{s}^{-1}(0) \cap \mathcal{M}\left(\theta_{1}, O_{a^{\prime}}\right)^{*}\right)
\end{aligned}
$$

and

(109)

$$
\begin{aligned}
\partial^{(1) \mathcal{M}}\left(O_{a}, \theta_{1}\right)^{*}= & \bigcup_{\left\{a^{\prime} \mid \mu\left(O_{a^{\prime}}\right)>\mu\left(\theta_{1}\right)\right\}}\left(\mathcal{M}\left(O_{a}, O_{a^{\prime}}\right)^{*} \times{O_{a^{\prime}}} \hat{\mathcal{M}}\left(O_{a^{\prime}}, \theta_{1}\right)^{*}\right) \\
& \bigcup_{\left\{b \mid \mu\left(O_{b}\right)<\mu\left(O_{a}\right)\right\}}\left(-\hat{\mathcal{M}}\left(O_{a}, O_{b}\right)^{*} \times{ }_{O_{b}} \mathcal{M}\left(O_{b}, \theta_{1}\right)^{*}\right) \\
& \cup \partial^{(1)}\left(\tilde{s}^{-1}(0) \cap \mathcal{M}\left(O_{a}, \theta_{0}\right)\right) \times \mathcal{M}\left(\theta_{0}, \theta_{1}\right) .
\end{aligned}
$$

Proof. By the results of Proposition 6.20, we have gluing maps

$$
\mathcal{M}\left(\theta_{0}, \theta_{1}\right) \times \bar{s}^{-1}(0) \rightarrow \mathcal{M}\left(\theta_{0}, a^{\prime}\right)
$$

and

$$
\bar{s}^{-1}(0) \times \mathcal{M}\left(\theta_{0}, \theta_{1}\right) \rightarrow \mathcal{M}\left(a, \theta_{1}\right)
$$

that are smooth embeddings. The dimension count then implies that these gluing maps are diffeomorphisms of $\bar{s}^{-1}(0)$ to a union of connected components of $\mathcal{M}\left(\theta_{0}, a^{\prime}\right)$ or $\mathcal{M}\left(a, \theta_{1}\right)$. Similarly, we have diffeomorphisms of $U(1)$-manifolds between $\tilde{s}^{-1}(0)$ and a union of connected components of $\mathcal{M}\left(\theta_{0}, O_{a^{\prime}}\right)$ or $\mathcal{M}\left(O_{a}, \theta_{1}\right)$, induced by the gluing maps

$$
\#: \mathcal{M}\left(\theta_{0}, \theta_{1}\right) \times \tilde{s}^{-1}(0) \rightarrow \mathcal{M}\left(\theta_{0}, O_{a^{\prime}}\right)
$$

and

$$
\#: \tilde{s}^{-1}(0) \times \mathcal{M}\left(\theta_{0}, \theta_{1}\right) \rightarrow \mathcal{M}\left(O_{a}, \theta_{1}\right) .
$$

Under the gluing maps, the image of

$$
\mathcal{M}^{b a l}\left(O_{a}, \theta_{0}\right) \cap \tilde{s}^{-1}(0) \quad \text { or } \quad \mathcal{M}^{b a l}\left(\theta_{1}, O_{a^{\prime}}\right) \cap \tilde{s}^{-1}(0)
$$

is a co-dimension 1 submanifold of $\mathcal{M}\left(\theta_{0}, O_{a^{\prime}}\right)$ or $\mathcal{M}\left(O_{a}, \theta_{1}\right)$. This submanifold actually lies in the interior of $\mathcal{M}\left(\theta_{0}, O_{a^{\prime}}\right)$ or $\mathcal{M}\left(O_{a}, \theta_{1}\right)$, and is not 
part of its boundary strata. This can be seen from the fact that the gluing maps (110) and (111) provide the collar structure around these smooth codimension one embedded submanifolds. In other words, this means that any sequence of solutions in $\mathcal{M}\left(\theta_{0}, O_{a^{\prime}}\right)$ or $\mathcal{M}\left(O_{a}, \theta_{1}\right)$ that converges to an element in the ideal boundary components (104) and (105) is in fact already convergent in the interior (top stratum) of $\mathcal{M}\left(\theta_{0}, O_{a^{\prime}}\right)$ or $\mathcal{M}\left(O_{a}, \theta_{1}\right)$. The only contribution of (104) and (105) to the actual boundary of the compactification then comes from the boundary points of

$$
\mathcal{M}\left(O_{a}, \theta_{0}\right) \cap \tilde{s}^{-1}(0)
$$

and

$$
\mathcal{M}\left(\theta_{1}, O_{a^{\prime}}\right) \cap \tilde{s}^{-1}(0) .
$$

This gives the formulae (108) and (109).

Similarly, we can analyze the boundary structure of $\mathcal{M}^{P}\left(\theta_{0}, O_{a}\right)$, in the presence of obstructions. We have the following result.

Theorem 6.22. For a generic choice of perturbation, the obstruction section

$$
\bar{s}: \mathcal{M}\left(\theta_{1}, a\right) \rightarrow \mathcal{L}_{\theta_{1}, a}=\mathcal{M}\left(\theta_{1}, O_{a}\right) \times_{U(1)} \mathbb{C}
$$

defines a codimension 2 submanifold $\bar{s}^{-1}(0) \subset \mathcal{M}\left(\theta_{1}, a\right)$, which corresponds to a $U(1)$-submanifold of co-dimension $2, \tilde{s}^{-1}(0) \subset \mathcal{M}\left(\theta_{1}, O_{a}\right)$, such that the gluing map

$$
\#: K \subset \mathcal{M}\left(\theta_{0}, \theta_{1}\right) \times \tilde{s}^{-1}(0) \rightarrow \mathcal{M}_{\sigma=1}^{P}\left(\theta_{0}, O_{a}\right)
$$

is a smooth embedding, for any compact set $K \subset \mathcal{M}\left(\theta_{0}, \theta_{1}\right) \times \tilde{s}^{-1}(0)$. Thus, the co-dimension one boundary strata of the compactification $\mathcal{M}^{P}\left(\theta_{0}, O_{a}\right)^{*}$ are given by

$$
\begin{aligned}
\partial^{(1)} \mathcal{M}^{P}\left(\theta_{0}, O_{a}\right)^{*}= & \bigcup_{a^{\prime}}\left(\mathcal{M}\left(\theta_{0}, O_{a^{\prime}}\right)^{*} \times O_{a^{\prime}} \mathcal{M}\left(O_{a^{\prime}}, O_{b}\right)^{*}\right) \\
& \bigcup \mathcal{M}\left(\theta_{0}, \theta_{1}\right) \times\left(\tilde{s}^{-1}(0) \cap \mathcal{M}\left(\theta_{1}, O_{a}\right)\right) \\
& \bigcup_{\left\{c \mid \mu\left(O_{c}\right) \geq \mu\left(\theta_{0}\right)\right\}}\left(\mathcal{M}^{P}\left(\theta_{0}, O_{c}\right)^{*} \times{ }_{O_{c}} \hat{\mathcal{M}}\left(O_{c}, O_{b}\right)^{*}\right) \\
& \bigcup_{\left\{c \mid \mu\left(O_{c}\right) \leq \mu\left(\theta_{0}\right)\right\}}\left(\hat{\mathcal{M}}\left(\theta_{0}, O_{c}\right)^{*} \times_{O_{c}} \mathcal{M}^{P}\left(O_{c}, O_{a}\right)^{*}\right)
\end{aligned}
$$

with the orientations given by the gluing theorem.

The proof follows from the analysis of the obstruction bundles and sections, as in the case of Proposition 6.20 and Theorem 6.21. 


\subsection{Proof of topological invariance.}

We gave an argument for the easy case of theorem 6.1, with maps $I, J$, and $H$ as in (76), (79), and (82). This gives us an isomorphism between the equivariant Floer homologies for any two choices $\left(g_{0}, \nu_{0}\right)$ and $\left(g_{1}, \nu_{1}\right)$ within the same chamber. Now we prove the general case

Proof of Theorem 6.1, Part II: the general case. In Section 6.1 and Section 6.2 , we analysed the boundary structure of the moduli spaces $\mathcal{M}\left(O_{a}, O_{a^{\prime}}\right)^{*}$ and $\mathcal{M}^{P}\left(O_{a}, O_{b}\right)^{*}$, in the case of two metrics and perturbations $\left(g_{0}, \nu_{0}\right)$ and $\left(g_{1}, \nu_{1}\right)$ in different chambers, connected by a path $\left(g_{t}, \nu_{t}\right)$ satisfying $S F\left(\partial_{\nu_{t}}^{g_{t}}\right)=-2$, and a path $\left(\tilde{g}_{t}, \tilde{\nu}_{t}\right)$, in the opposite direction, satisfying $S F\left(\partial_{\tilde{\nu}_{t}}^{\tilde{g}_{t}}\right)=-S F\left(\partial_{\nu_{t}}^{g_{t}}\right)=2$. We assume throughout the discussion that the metrics $\left(g_{0}, \nu_{0}\right)$ and $\left(g_{1}, \nu_{1}\right)$ are "close enough", on the two sides of the wall. We can assume, similarly, that the paths $\left(g_{t}, \nu_{t}\right)$ and $\left(\tilde{g}_{t}, \tilde{\nu}_{t}\right)$ are also close enough.

Recall that we perform a shift of grading in the complex $C_{k U(1)}\left(Y, g_{1}, \nu_{1}\right)$ by setting $\mu\left(\theta_{1}\right)=-S F\left(\partial_{\nu_{t}}^{g_{t}}\right)$, where $S F\left(\partial_{\nu_{t}}^{g_{t}}\right)$ is the spectral flow of the Dirac operator along the path of reducible solutions $\left[\nu_{t}, 0\right]$.

The analysis of the obstruction bundles in Section 6.2 implies that the boundary structure of the moduli spaces $\mathcal{M}\left(O_{a}, O_{a^{\prime}}\right)$ and $\mathcal{M}^{P}\left(O_{a}, O_{b}\right)$ is modified by the presence of the zeroes of the obstruction sections. This difference determines suitable correction terms for the maps $I$ and $H$, so that the argument of Theorem 6.1 can be adapted to this general case.

Remark 6.23. The moduli space $\mathcal{M}\left(\theta_{1}, \theta_{0}\right)$, as a $U(1)$-manifold, consists of a disk, containing the fixed point $x_{1}=\left[\mathbb{A}_{1}, 0\right]$ (the unique solution of $d * \mathbb{A}_{1}=0$ and $d^{+} \mathbb{A}_{1}=\tilde{\mu}_{t}$ ), and with boundary a circle, given either by a component

$$
\mathcal{M}\left(\theta_{1}, O_{a}\right) \times_{O_{a}} \hat{\mathcal{M}}\left(O_{a}, \theta_{0}\right)
$$

with $\mu\left(O_{a}\right)-\mu\left(\theta_{0}\right)=1$, or by a component

$$
\hat{\mathcal{M}}\left(\theta_{1}, O_{a}^{\prime}\right) \times_{O_{a}^{\prime}} \mathcal{M}\left(O_{a}^{\prime}, \theta_{0}\right),
$$

with $\mu\left(O_{a}^{\prime}\right)-\mu\left(\theta_{0}\right)=0$. Thus, we have the following identity that counts the boundary components of the framed monopole moduli space $\mathcal{M}\left(\theta_{1}, \theta_{0}\right)$ :

$$
\sum_{a_{(1)}} n_{\theta_{1} a_{(1)}} n_{a_{(1)} \theta_{0}}+\sum_{a_{(0)}^{\prime}} n_{\theta_{1} a_{(0)}^{\prime}} n_{a_{(0)}^{\prime} \theta_{0}}=1
$$

The sum is over all $O_{a_{(1)}}$ in $\mathcal{M}^{0}\left(Y, g_{0}, \nu_{0}\right)$, and $O_{a_{(0)}^{\prime}}$ in $\mathcal{M}^{0}\left(Y, g_{1}, \nu_{1}\right)$ of (shifted) index $\mu\left(O_{a_{(1)}}\right)-\mu\left(\theta_{0}\right)=1$ and $\mu\left(O_{a_{(0)}^{\prime}}\right)-\mu\left(\theta_{0}\right)=0$, respectively. 
In particular, notice that we can derive the observation of Remark 6.23 if we make explicit use here of the information on the local structure of the moduli space $\mathcal{M}^{0}\left(Y, g_{t}, \nu_{t}\right)$ of critical orbits, as the metric $\left(g_{t}, \nu_{t}\right)$ approaches the wall, This is derived in Section 7.3.

We know that two possibilities arise: either an irreducible orbit $O_{a}$, with $\mu\left(O_{a}\right)-\mu\left(\theta_{0}\right)=1$, disappears into the reducible as the metric and perturbation $\left(g_{t}, \nu_{t}\right)$ hits the wall, or an irreducible orbit $O_{a^{\prime}}$ with $\mu\left(O_{a^{\prime}}\right)-$ $\mu\left(\theta_{0}\right)=0$ arises from the reducible, as $\left(g_{t}, \nu_{t}\right)$ hits the wall. In the first case, the disk of Remark 6.23 has boundary the circle

$$
\mathcal{M}\left(\theta_{1}, O_{a}\right) \times O_{a} \hat{\mathcal{M}}\left(O_{a}, \theta_{0}\right)
$$

and in the second case it has boundary the circle

$$
\hat{\mathcal{M}}\left(\theta_{1}, O_{a}^{\prime}\right) \times_{O_{a}^{\prime}} \mathcal{M}\left(O_{a}^{\prime}, \theta_{0}\right) .
$$

The relation (113) then yields the separate identities

$$
\# \mathcal{M}\left(\theta_{1}, O_{a}\right)=n_{\theta_{1} a}=1 \text { and } \# \hat{\mathcal{M}}\left(O_{a}, \theta_{0}\right)=n_{a \theta_{0}}=1,
$$

for $O_{a}$ the unique orbit that hits the reducible, and

$$
\sum_{a_{(1)} \neq a} n_{\theta_{1} a_{(1)}} n_{a_{(1)} \theta_{0}}+\sum_{a_{(0)}^{\prime}} n_{\theta_{1} a_{(0)}^{\prime}} n_{a_{(0)}^{\prime} \theta_{0}}=0
$$

for $\mu\left(O_{a_{(1)}}\right)-\mu\left(\theta_{0}\right)=1$ and $\mu\left(O_{a_{(0)}^{\prime}}\right)-\mu\left(\theta_{0}\right)=0$. The case of $O_{a^{\prime}}$ is analogous.

The moduli space $\mathcal{M}\left(\theta_{1}, \theta_{0}\right)$, containing this separate component with a fixed point, is one of the differences with respect to the picture for metrics and perturbations within the same chamber. Another essential difference is, of course, the presence of the singular moduli space $\mathcal{M}\left(\theta_{0}, \theta_{1}\right)$. We have already seen, in the analysis of the obtructions how this moduli space plays an essential role. However, what we wish to point out here is that the basic asymmetry between the moduli spaces $\mathcal{M}\left(\theta_{0}, \theta_{1}\right)$ and $\mathcal{M}\left(\theta_{1}, \theta_{0}\right)$ is what calls for correction terms in the maps $I$ and $H$, but not in the map $J$.

In fact, consider first the action of the map $J$, defined as in (79) on the generator $\Omega^{n} \otimes 1_{\theta_{1}}$ in the Floer complex for $\left(g_{1}, \nu_{1}\right)$. We have

$$
J\left(\Omega^{n} \otimes 1_{\theta_{1}}\right)=\sum_{a_{(1)}} n_{\theta_{1} a_{(1)}} \Omega^{n} \otimes 1_{a_{(1)}}
$$

with the sum over all $O_{a_{(1)}}$ with $\mu\left(O_{a_{(1)}}\right)-\mu\left(\theta_{0}\right)=1$. 
Notice that the existence of the extra disk component in the boundary of $\mathcal{M}\left(\theta_{1}, \theta_{0}\right)$, as in remark 6.23 , does not affect the identity $J D-D J=0$, in fact, even though now the count of boundary terms in $\mathcal{M}\left(\theta_{0}, \theta_{1}\right)$ satisfies (113), we still do get

$$
\left\langle\Omega^{n} \otimes 1_{\theta_{0}}, J D-D J\left(\Omega^{n} \otimes 1_{\theta_{1}}\right)\right\rangle=0 .
$$

In fact, the fibrations

$$
\begin{aligned}
& \mathcal{M}\left(O_{a_{(0)}^{\prime}}, \theta_{0}\right) \rightarrow \theta_{0}, \\
& \hat{\mathcal{M}}\left(O_{a_{(1)}}, \theta_{0}\right) \rightarrow \theta_{0},
\end{aligned}
$$

have 1-dimensional fibers, and the pushforward of a zero-form is trivial. The identity $J D-D J=0$ at all the other components follows the argument given in the proof of the easy case of Theorem 6.1, without any modification.

Now, instead, consider the case of the map $I$ acting on $\Omega^{n} \otimes 1_{\theta_{0}}$. The first difference we notice, with respect to the model case of metrics and perturbations in the same chamber, is that the moduli space $\mathcal{M}\left(\theta_{0}, \theta_{1}\right)$ consisting of the unique point $x_{0}=\left[\mathbb{A}_{0}, 0\right]$ defines a non-trivial "pull-back push-forward" acting on the zero form $1_{\theta_{0}}$. To account for this moduli space, we have to assume the existence here of an extra component of the map $I$ connecting $\Omega^{n} \otimes 1_{\theta_{0}}$ to $\Omega^{n-1} \otimes 1_{\theta_{1}}$, where the drop of degree in $\Omega$ accounts for the change of grading of the reducible point, so that the map $I$ can be of degree zero. Thus, we have a new component

$$
\left\langle\Omega^{n-1} \otimes 1_{\theta_{1}}, I\left(\Omega^{n} \otimes 1_{\theta_{0}}\right)\right\rangle=1 .
$$

Notice that the necessity of the additional term (115) in the map $I$ can be made clear by looking at the following example.

Example. Consider a model case where the moduli space $\mathcal{M}^{0}\left(g_{0}, \nu_{0}\right)$ consists solely of the fixed point $\theta_{0}$ and of an irreducible orbit $O_{a}$ in degree one. The Floer complex for $\left(g_{0}, \nu_{0}\right)$ then has generators $\Omega^{n} \otimes 1_{\theta_{0}}$ in even degrees $p=2 n \geq 0, \Omega^{n} \otimes \eta_{a}$ in odd degrees $p=2 n+1 \geq 1$, and $\Omega^{n-1} \otimes 1_{a}$ in even degrees $p=2 n \geq 2$. The boundary operator $D$ has a component

$$
\Omega^{n} \otimes \eta_{a} \mapsto-\Omega^{n-1} \otimes 1_{a}+n_{a 0} \Omega^{n} \otimes 1_{\theta_{0}} .
$$

In the wall crossing with $\mu\left(\theta_{0}\right)-\mu\left(\theta_{1}\right)=-2$, the orbit $O_{a}$ disappears into the reducible, and the Floer complex for $\left(g_{1}, \nu_{1}\right)$ only has the fixed point as generator. In this case, we have the equivariant Floer homology

$$
H F_{p, U(1)}^{S W}\left(Y,\left(g_{0}, \nu_{0}\right)\right)= \begin{cases}\mathbb{R}\left[\Omega^{n-1} \otimes 1_{a}\right] & p=2 n \geq 2 \\ 0 & \text { otherwise }\end{cases}
$$


with $\left[\Omega^{n-1} \otimes 1_{a}\right]=\left[\Omega^{n} \otimes 1_{\theta_{0}}\right]$ for all $n \geq 1$, and $\left[1 \otimes 1_{\theta_{0}}\right]=0$. The Floer complex for $\left(g_{1}, \nu_{1}\right)$ has $\Omega^{n} \otimes 1_{\theta_{1}}$ as unique generators, and no boundary components, hence the Floer homology is

$$
H F_{p, U(1)}^{S W}\left(Y,\left(g_{1}, \nu_{1}\right)\right)=\left\{\begin{array}{lr}
\mathbb{R}\left[\Omega^{n} \otimes 1_{\theta_{1}}\right] & p=2 n+2 \geq 2 \\
0 & \text { otherwise }
\end{array}\right.
$$

after degree shift. The map $I$ that maps $\Omega^{n} \otimes 1_{\theta_{0}} \rightarrow \Omega^{n-1} \otimes 1_{\theta_{1}}$ gives the desired isomorphism. Notice that such examples can in fact be realized geometrically, for instance when considering a metric of positive scalar curvature on $S^{3}$ as $g_{1}$.

It becomes then clear that, if we change the map $I$ with an extra component as in (115), to account for the moduli space $\mathcal{M}\left(\theta_{0}, \theta_{1}\right)$, we need to add further correction terms to the original map $I$ on other generators, so that the identity $I D-D I=0$ continues to be satisfied. In assigning the necessary correction terms, we need to take into account the different structure of the compactification of the moduli spaces $\mathcal{M}\left(O_{a}, O_{a^{\prime}}\right)$, with the boundary strata (108) and (109). Studying the boundary information of $\mathcal{M}\left(\theta_{0}, a_{(-2)}^{\prime}\right)$, we know that there is a contribution of the singular gluing from the monopoles in

$$
s^{-1}(0) \cap \mathcal{M}\left(\theta_{1}, a_{(-2)}^{\prime}\right)
$$

which contributes to the expression

$$
(I D-D I)\left(\Omega^{n} \otimes 1_{\theta_{0}}\right),
$$

in addition to the ordinary components $n_{\theta_{0} a_{(-1)}^{\prime}} \Omega^{n} \otimes 1_{a_{(-1)}^{\prime}}$, where $n_{\theta_{0} a_{(-1)}^{\prime}}$ effectively counts the monopoles from the zeros of the obstruction section over $\mathcal{M}\left(\theta_{1}, a_{(-1)}^{\prime}\right)$. This is precisely the correction term which is needed in order to obtain the identity $I D-D I=0$ once we take into account the presence of the component (115) originates from the presence of the moduli space $\mathcal{M}\left(\theta_{0}, \theta_{1}\right)$ consisting of the unique point $x_{0}=\left[\mathbb{A}_{0}, 0\right]$.

Similarly, we need to introduce correction terms to the map $H$ so that it continues to be a chain homotopy, satisfying $i d-J I=D H+H D$, with respect to the modified map $I$ and with the modified structure of the compactification of $\mathcal{M}^{P}\left(O_{a}, O_{b}\right)$.

In order to introduce the correction terms for the maps $I$ and $H$, we need a preliminary discussion on some identities derived from the counting of zeroes of the obstruction sections. 
Consider the case of the 2-dimensional moduli space $\mathcal{M}\left(a, \theta_{0}\right)$, with $a$ an irreducible critical point of index 2 and $\theta_{0}$ the unique reducible point.

In Section 5.3, in Lemma 5.7, we described the invariant $m_{a c}$ as the relative Euler class of the complex line bundle $\mathcal{L}_{a c}$ over $\mathcal{M}(a, c)$. Consider now the case of the 2-dimensional moduli space $\mathcal{M}\left(a, \theta_{0}\right)$, with $O_{a}$ a free orbit of index 2 and $\theta_{0}$ the fixed point in $\mathcal{M}^{0}\left(Y, g_{0}, \nu_{0}\right)$. We also introduced the invariant $m_{a \theta_{0}}$ in Section 5.3, as as the relative Euler class of the associated complex line bundle over $\mathcal{M}\left(a, \theta_{0}\right)$, according to Lemma 5.9 and Lemma 5.10. As we point out in the following Remark, we can use the obstruction section to obtain the necessary trivialization and compute the invariant $m_{a \theta_{0}}$ as relative Euler class of $\mathcal{L}_{a \theta_{0}}$.

Remark 6.24. We use the same choice of trivialization $s_{a b}$ over $\mathcal{M}(a, b)$, for all the free orbits $O_{a}$ and $O_{b}$ of relative index 1, as determined by the trivialization $\varphi$ of Lemma 5.7. For $\mathcal{M}\left(b, \theta_{0}\right)$ with $\mu\left(O_{b}\right)-\mu\left(\theta_{0}\right)=1$, we set $s_{b, \theta_{0}} \neq 0$ to be the obstruction section

$$
s_{b, \theta_{0}}=\bar{s}: \mathcal{M}\left(b, \theta_{0}\right) \rightarrow \mathcal{M}\left(O_{b}, \theta_{0}\right) \times_{U(1)} \operatorname{Coker}\left(\mathcal{L}_{x}\right) \cong \mathcal{L}_{b \theta_{0}} .
$$

We then set $s_{a, \theta_{0}}$ over $\mathcal{M}\left(a, \theta_{0}\right)$, with $\mu\left(O_{a}\right)-\mu\left(\theta_{0}\right) \geq 2$ to be the obstruction section

$$
s_{a, \theta_{0}}=\bar{s}: \mathcal{M}\left(a, \theta_{0}\right) \rightarrow \mathcal{M}\left(O_{a}, \theta_{0}\right) \times_{U(1)} \operatorname{Coker}\left(\mathcal{L}_{x}\right) \cong \mathcal{L}_{a \theta_{0}} .
$$

This choice satisfies the requirement of the class of sections specified above. Over a moduli spaces $\mathcal{M}\left(a, \theta_{0}\right)$, with $\mu\left(O_{a}\right)-\mu\left(\theta_{0}\right) \geq 2$, then the obstruction section

$$
s_{a, \theta_{0}}=\bar{s}: \mathcal{M}\left(a, \theta_{0}\right) \rightarrow \mathcal{M}\left(O_{a}, \theta_{0}\right) \times_{U(1)} \operatorname{Coker}\left(\mathcal{L}_{x}\right) \cong \mathcal{L}_{a \theta_{0}}
$$

is homotopic to

$$
\pi_{1}^{*} s_{a b} \otimes \pi_{2}^{*} s_{b, \theta_{0}}
$$

over all the submanifolds

$$
\mathcal{M}(a, b) \times \mathcal{M}\left(b, \theta_{0}\right)
$$

of $\mathcal{M}\left(a, \theta_{0}\right)$, where we identify

$$
\mathcal{L}_{a, \theta_{0}}=\pi_{1}^{*} \mathcal{L}_{a b} \otimes \pi_{2}^{*} \mathcal{L}_{b, \theta_{0}},
$$

with $\pi_{1}$ and $\pi_{2}$ the projections on the two factors in

$$
\mathcal{M}(a, b) \times \mathcal{M}\left(b, \theta_{0}\right),
$$


with $\mu\left(O_{a}\right)>\mu\left(O_{b}\right)>\mu\left(\theta_{0}\right)$, and section $s_{b, \theta_{0}}$ is the obstruction section over $\mathcal{M}\left(b, \theta_{0}\right)$, and the section $s_{a b}$ is a transverse section of the line bundle

$$
\mathcal{L}_{a b}=\mathcal{M}\left(O_{a}, O_{b}\right) \times_{U(1)} \mathbb{C}
$$

over $\mathcal{M}(a, b)$ as discussed in Section 5.3.

In summary, we have obtained the following lemma which claims that the relative Euler class $m_{a_{(2)} \theta_{0}}$ and $m_{\theta_{1} a_{(-1)}^{\prime}}$ can be calculated by counting the zeros of the obstruction section over $\mathcal{M}\left(a_{(2)}, \theta_{0}\right)$ and $\mathcal{M}\left(\theta_{1}, a_{(-1)}^{\prime}\right)$ respectively.

Lemma 6.25. For $\mu\left(a_{(2)}\right)-\mu\left(\theta_{0}\right)=2$, the relative Euler class $m_{a_{(2)} \theta_{0}}$ is given by

$$
\# s_{a_{(2)} \theta_{0}}^{-1}(0)
$$

where $s_{a_{(2)} \theta_{0}}$ is the obstruction section over $\mathcal{M}\left(a_{(2)}, \theta_{0}\right)$, similarly, for $\mu\left(\theta_{1}\right)-\mu\left(a_{(-1)}^{\prime}\right)=3$, the relative Euler class $m_{\theta_{1} a_{(-1)}^{\prime}}$ is given by

$$
\# s_{\theta_{0} a_{(-1)}^{\prime}}^{-1}(0)
$$

where $s_{\theta_{0} a_{(-1)}^{\prime}}$ is the obstruction section over $\mathcal{M}\left(\theta_{1}, a_{(-1)}^{\prime}\right)$.

As a consequence of Remark 6.24 and Lemma 6.25, we have the following identities.

Lemma 6.26. Let $O_{a_{(p)}}$ denote a free orbit in the moduli space $\mathcal{M}^{0}\left(Y, g_{0}, \nu_{0}\right)$, of index $\mu\left(O_{a_{(p)}}\right)-\mu\left(\theta_{0}\right)=p$. Let $O_{a_{(q)}^{\prime}}$ denote a free orbit in the moduli space $\mathcal{M}^{0}\left(Y, g_{1}, \nu_{1}\right)$, of (shifted) index $\mu\left(O_{a_{(q)}^{\prime}}\right)-\mu\left(\theta_{0}\right)=q$. We have the following properties.

1. Consider the obstruction bundle over $\mathcal{M}\left(a_{(2)}, \theta_{0}\right)$. The counting of the zeroes of the obstruction, that is, of the flowlines in $\mathcal{M}\left(a_{(2)}, \theta_{0}\right)$ glued to the singular reducible $x=\left[\mathbb{A}^{0}, 0\right]$ in $\mathcal{M}\left(\theta_{0}, \theta_{1}\right)$, is given by

$$
m_{a_{(2)} \theta_{0}}=\#\left(\bar{s}^{-1}(0) \cap \mathcal{M}\left(a_{(2)}, \theta_{0}\right)\right) .
$$

This satisfies

$$
\sum_{a_{(1)}} m_{a_{(3)} a_{(1)}} n_{a_{(1)} \theta_{0}}-\sum_{a_{(2)}} n_{a_{(3)} a_{(2)}} m_{a_{(2)} \theta_{0}}=0 .
$$


2. The counting of the zeroes of the obstruction section $\bar{s}$ of the obstruction bundle over $\mathcal{M}\left(\theta_{1}, a_{(-1)}^{\prime}\right)$, which counts the gluing of the singular reducible $x=\left[\mathbb{A}^{0}, 0\right]$ in $\mathcal{M}\left(\theta_{0}, \theta_{1}\right)$ to flowlines in $\mathcal{M}\left(\theta_{1}, a_{(-1)}^{\prime}\right)$, is given by

$$
m_{\theta_{1} a_{(-1)}^{\prime}}=\#\left(\bar{s}^{-1}(0) \cap \mathcal{M}\left(\theta_{1}, a_{(-1)}^{\prime}\right)\right) .
$$

This satisfies

$$
\sum_{a_{(-1)}^{\prime}} m_{\theta_{1} a_{(-1)}^{\prime}} n_{a_{(-1)}^{\prime} a_{(-2)}^{\prime}}-\sum_{a_{(0)}^{\prime}} n_{\theta_{1} a_{(0)}^{\prime}} m_{a_{(0)}^{\prime} a_{(-2)}^{\prime}}=0 .
$$

Proof. In this case, we know that, for dimensional reasons, we have

$$
\bar{s}^{-1}(0) \cap \mathcal{M}\left(a_{(1)}, \theta_{0}\right)=\emptyset .
$$

Thus, by the previous discussion, and the results of Lemma 5.9, Lemma 5.10 , and Remark 6.24, we know that the boundary of the 1-dimensional manifold $\left(\bar{s}^{-1}(0) \cap \mathcal{M}\left(a_{(3)}, \theta_{0}\right)\right)$ consists of the set

$$
\begin{gathered}
\bigcup_{a_{(2)}} \hat{\mathcal{M}}\left(a_{(3)}, a_{(2)}\right) \times\left(\bar{s}^{-1}(0) \cap \mathcal{M}\left(a_{(2)}, \theta_{0}\right)\right) \\
\cup \bigcup_{a_{(1)}}\left(-s_{a_{(2)} a_{(1)}}^{-1}(0) \cap\left(\mathcal{M}\left(a_{(2)}, a_{(1)}\right) \times \hat{\mathcal{M}}\left(a_{(1)}, \theta_{0}\right)\right)\right) .
\end{gathered}
$$

This implies that we have the identity

$$
\sum_{a_{(1)}} m_{a_{(3)} a_{(1)}} n_{a_{(1)} \theta_{0}}-\sum_{a_{(2)}} n_{a_{(3)} a_{(2)}} m_{a_{(2)} \theta_{0}}=0,
$$

with the sign denoting the different orientation. The remaining case is analogous.

Now we define the necessary modifications to the maps $I$ and $H$.

Definition 6.27. We modify the maps $I, H$ on $\Omega^{n} \otimes 1_{\theta_{0}}$ and $\Omega^{n-1} \otimes 1_{a_{(1)}}$ as follows:

1.

$$
\begin{aligned}
I\left(\Omega^{n} \otimes 1_{\theta_{0}}\right)= & \Omega^{n-1} \otimes 1_{\theta_{1}} \\
& +\sum_{a_{(-1)}^{\prime}} n_{\theta_{0} a_{(-1)}^{\prime}} \Omega^{n} \otimes 1_{a_{(-1)}^{\prime}} \\
& +\sum_{a_{(0)}^{\prime}} n_{\theta_{1} a_{(0)}^{\prime}} \Omega^{n} \otimes \eta_{a_{(0)}^{\prime}}
\end{aligned}
$$


2.

$$
\begin{aligned}
I\left(\Omega^{n-1} \otimes 1_{a_{(1)}}\right)= & n_{a_{(1)} \theta_{0}} \Omega^{n-1} \otimes 1_{\theta_{1}} \\
& +\sum_{a_{(1)}^{\prime}} n_{a_{(1)} a_{(1)}^{\prime}} \Omega^{n-1} \otimes 1_{a_{(1)}^{\prime}}
\end{aligned}
$$

3.

$$
\begin{aligned}
H\left(\Omega^{n} \otimes 1_{\theta_{0}}\right)= & \sum_{a_{(0)}} n_{\theta_{0} a_{(0)}}^{P} \Omega^{n} \otimes 1_{a(0)} \\
& +\sum_{a_{(1)}} n_{\theta_{1} a_{(1)}} \Omega^{n} \otimes \eta_{a_{(1)}}
\end{aligned}
$$

In order to show that the relations $I D=D I$ and $i d-J I=D H+H D$ are still satisfied, we only need to check explicitly all the terms that are directly affected by the presence of the correction terms.

Lemma 6.28. We have

1. $I D=D I$ on $\Omega^{n} \otimes 1_{\theta_{0}}$ and $\Omega^{n-1} \otimes 1_{a_{(1)}}$;

2. $I D=D I$ on $\Omega^{n} \otimes \eta_{a_{(3)}}$;

3. $I D=D I$ on $\Omega^{n} \otimes \eta_{a_{(1)}}$;

Proof. We prove the claim by direct analysis of the boundary strata of the various moduli spaces involved. We have

$$
I D\left(\Omega^{n} \otimes 1_{\theta_{0}}\right)=\sum_{a_{(-2)}} n_{\theta_{0} a_{(-2)}} n_{a_{(-2)} a_{(-2)}^{\prime}} \Omega^{n} \otimes 1_{a_{(-2)}^{\prime}}
$$

and

$$
\begin{aligned}
D I\left(\Omega^{n} \otimes 1_{\theta_{0}}\right)= & -\sum_{a_{(-1)}^{\prime}} n_{\theta_{0} a_{(-1)}^{\prime}} n_{a_{(-1)}^{\prime} a_{(-2)}^{\prime}} \Omega^{n} \otimes 1_{a_{(-2)}^{\prime}} \\
& +\sum_{a_{(0)}^{\prime}} n_{\theta_{1} a_{(0)}^{\prime}} m_{a_{(0)}^{\prime} a_{(-2)}^{\prime}} \Omega^{n} \otimes 1_{a_{(-2)}^{\prime}}
\end{aligned}
$$

The first claim then follows, since we know that the co-dimension one boundary of $\mathcal{M}\left(\theta_{0}, O_{a_{(-2)}^{\prime}}\right)$ consists of

$$
\bigcup_{a_{(-2)}} \hat{\mathcal{M}}\left(\theta_{0}, O_{a_{(-2)}}\right) \times_{O_{a_{(-2)}}} \mathcal{M}\left(O_{a_{(-2)}}, O_{a_{(-2)}^{\prime}}\right)
$$




$$
\begin{gathered}
\cup \bigcup_{a_{(-1)}^{\prime}} \mathcal{M}\left(\theta_{0}, O_{a_{(-1)}^{\prime}}\right) \times \hat{\mathcal{M}}\left(O_{a_{(-1)}}, O_{a_{(-2)}^{\prime}}\right) \\
\cup \mathcal{M}\left(\theta_{0}, \theta_{1}\right) \times\left(\tilde{s}^{-1}(0) \cap\left(\hat{\mathcal{M}}\left(\theta_{1}, O_{a_{(0)}^{\prime}}\right) \times_{O_{a_{(0)}^{\prime}}} \mathcal{M}\left(O_{a_{(0)}^{\prime}}, O_{a_{(-2)}^{\prime}}\right)\right) .\right.
\end{gathered}
$$

In fact, according to Theorem 6.21, we have boundary strata as in (108),

$$
\mathcal{M}\left(\theta_{0}, \theta_{1}\right) \times \partial^{(1)}\left(\tilde{s}^{-1}(0) \cap \mathcal{M}\left(\theta_{1}, O_{a_{(-2)}^{\prime}}\right)^{*}\right) .
$$

Here $\tilde{s}$ is the obstruction section. By Remark 6.24, we have

$$
\begin{aligned}
& \partial^{(1)}\left(\tilde{s}^{-1}(0) \cap \mathcal{M}\left(\theta_{1}, O_{a_{(-2)}^{\prime}}\right)^{*}\right) \\
= & \tilde{s}^{-1}(0) \cap\left(\bigcup_{a_{(0)}^{\prime}} \hat{\mathcal{M}}\left(\theta_{1}, O_{a_{(0)}^{\prime}}\right)^{*} \times_{O_{a_{(0)}^{\prime}}} \mathcal{M}\left(O_{a_{(0)}^{\prime}}, O_{a_{(-2)}^{\prime}}\right)^{*}\right) \\
& \cup \tilde{s}^{-1}(0) \cap\left(\bigcup_{a_{(-1)}^{\prime}} \hat{\mathcal{M}}\left(\theta_{1}, O_{a_{(-1)}^{\prime}}\right)^{*} \times_{O_{a_{(-1)}^{\prime}}} \mathcal{M}\left(O_{a_{(-1)}^{\prime}}, O_{a_{(-2)}^{\prime}}\right)^{*}\right) .
\end{aligned}
$$

Note that the coefficient $n_{\theta_{0} a_{(-1)}^{\prime}}$ effectively counts the monopoles from the zeros of the obstruction section over $\mathcal{M}\left(\theta_{1}, a_{(-1)}^{\prime}\right)$, so we are left with the remaining counting,

$$
\sum_{a_{(0)}^{\prime}} n_{\theta_{1} a_{(0)}^{\prime}} m_{a_{(0)}^{\prime} a_{(-2)}^{\prime}} \Omega^{n} \otimes 1_{a_{(-2)}^{\prime}},
$$

which proves the claim.

In order to prove Claim (2), we compute

$$
I D\left(\Omega^{n} \otimes 1_{a_{(1)}}\right)=-\sum_{a_{(0)}} n_{a_{(1)} a_{(0)}} n_{a_{(0)} a_{(0)}^{\prime}} \Omega^{n} \otimes 1_{a_{(0)}^{\prime}}
$$

and

$$
D I\left(\Omega^{n} \otimes 1_{a_{(1)}}\right)=-\sum_{a_{(1)}^{\prime}} n_{a_{(1)} a_{(1)}^{\prime}} n_{a_{(1)}^{\prime} a_{(0)}^{\prime}} \Omega^{n} \otimes 1_{a_{(0)}^{\prime}}+n_{a_{(1)} \theta_{0}} n_{\theta_{1} a_{(0)}^{\prime}} \Omega^{n} \otimes 1_{a_{(0)}^{\prime}}
$$

Then the claim follows from the boundary structure of $\mathcal{M}\left(a_{(1)}, a_{(0)}^{\prime}\right)$. The zeroes of the obstruction section contribute the term

$$
n_{a_{(1)} \theta_{0}} n_{\theta_{1} a_{(0)}^{\prime}}
$$

in the counting of the boundary points.

The argument for Claims (3) and (4) is analogous.

Now we need to check the effect of the correction terms on the identity $1-J I=H D+H D$. 
Lemma 6.29. We have the identity $1-J I=D H+H D$ on $\Omega^{n} \otimes 1_{\theta_{0}}$.

Proof. By direct calculation, we obtain

$$
\begin{aligned}
& (1-J I)\left(\Omega^{n} \otimes 1_{\theta_{0}}\right) \\
= & \Omega^{n} \otimes 1_{\theta_{0}}-J\left(\Omega^{n-1} \otimes 1_{\theta_{1}}+\sum_{a_{(-1)}^{\prime}} n_{\theta_{0} a_{(-1)}^{\prime}} \Omega^{n} \otimes 1_{a_{(-1)}^{\prime}}\right. \\
& \left.+\sum_{a_{(0)}^{\prime}} n_{\theta_{1} a_{(0)}^{\prime}} \Omega^{n} \otimes \eta_{a_{(0)}^{\prime}}\right) \\
= & \Omega^{n} \otimes 1_{\theta_{0}}-\sum_{a_{(1)}} n_{\theta_{1} a_{(1)}} \Omega^{n-1} \otimes 1_{a_{(1)}} \\
& -\left(\sum_{a_{(-1)}^{\prime}} n_{\theta_{0} a_{(-1)}^{\prime}} n_{a_{(-1)}^{\prime} a_{(-1)}}+\sum_{a_{(0)}^{\prime}} n_{\theta_{1} a_{(0)}^{\prime}} m_{a_{(0)}^{\prime} a_{(-1)}}\right) \Omega^{n} \otimes 1_{a_{(-1)}} \\
& -\sum_{a_{(0)}^{\prime}} n_{\theta_{1} a_{(0)}^{\prime}} n_{a_{(0)}^{\prime} a_{(0)}} \Omega^{n} \otimes \eta_{a_{(0)}}-\sum_{a_{(0)}^{\prime}} n_{\theta_{1} a_{(0)}^{\prime}} n_{a_{(0)}^{\prime} \theta_{0}} \Omega^{n} \otimes 1_{\theta_{0}}
\end{aligned}
$$

We also get

$$
\begin{aligned}
& (D H+H D)\left(\left(\Omega^{n} \otimes 1_{\theta_{0}}\right)\right. \\
= & D\left(\sum_{a_{(0)}} n_{\theta_{0} a_{(0)}}^{P} \Omega^{n} \otimes 1_{a(0)}+\sum_{a_{(1)}} n_{\theta_{1} a_{(1)}} \Omega^{n} \otimes \eta_{a_{(1)}}\right) \\
& +H\left(\sum_{a_{(-2)}} n_{\theta_{0} a_{(-2)}} \Omega^{n} \otimes 1_{a_{(-2)}}\right) \\
= & \sum_{a_{(-1)}}\left(\sum_{a_{(1)}} n_{\theta_{1} a_{(1)}} m_{a_{(1)} a_{(-1)}}-\sum_{a_{(0)}} n_{\theta_{0} a_{(0)}}^{P} n_{a_{(0)} a_{(-1)}}\right. \\
& \left.-\sum_{a_{(-2)}} n_{\theta_{0} a_{(-2)}} n_{a_{(-2)}}^{P} a_{(-1)}\right) \Omega^{n} \otimes 1_{a(-1)} \\
& +\sum_{a_{(1)}, a_{(0)}} n_{\theta_{1} a_{(1)}} n_{a_{(1)} a_{(0)}} \Omega^{n} \otimes \eta_{a_{(0)}}-\sum_{a_{(1)}} n_{\theta_{1} a_{(1)}} \Omega^{n-1} \otimes 1_{a_{(1)}} \\
& +\sum_{a_{(1)}} n_{\theta_{1} a_{(1)}} n_{a_{(1)} \theta_{0}} \Omega^{n} \otimes 1_{\theta_{0}} .
\end{aligned}
$$

Let us first check the coefficient of $\Omega^{n} \otimes 1_{\theta_{0}}$. Equating the coefficients of $\Omega^{n} \otimes 1_{\theta_{0}}$ in the two expressions above yields the identity

$$
\sum_{a_{(1)}} n_{\theta_{1} a_{(1)}} n_{a_{(1)} \theta_{0}}+\sum_{a_{(1)}^{\prime}} n_{\theta_{1} a_{(0)}^{\prime}} n_{a_{(0)}^{\prime} \theta_{0}}=1
$$

This identity is satisfied, since it is exactly the counting of (113).

We then check the coefficient of $\Omega^{n} \otimes \eta_{a_{(0)}}$. We need to prove the identity

$$
\sum_{a_{(1)}} n_{\theta_{1} a_{(1)}} n_{a_{(1)} a_{(0)}}+\sum_{a_{(0)}^{\prime}} n_{\theta_{1} a_{(0)}^{\prime}} n_{a_{(0)}^{\prime} a_{(0)}}=0 .
$$

This follows from the boundary structure of $\mathcal{M}\left(\theta_{1}, O_{a_{(0)}}\right)^{*}$, given by

$$
\bigcup_{a_{(1)}} \mathcal{M}\left(\theta_{1}, O_{a_{(1)}}\right)^{*} \times_{O_{a_{(1)}}} \hat{\mathcal{M}}\left(O_{a_{(1)}}, O_{a_{(0)}}\right)^{*}
$$




$$
\left.\cup \bigcup_{a_{(0)}^{\prime}} \hat{\mathcal{M}} \theta_{1}, O_{a_{(0)}^{\prime}}\right)^{*} \times O_{a_{(0)}^{\prime}} \mathcal{M}\left(O_{a_{(0)}^{\prime}}, O_{a_{(0)}}\right)^{*}
$$

In order to compare the coefficients of $\Omega^{n} \otimes 1_{a(-1)}$, we need to prove the identity

$$
\begin{aligned}
& \sum_{a_{(-1)}^{\prime}} n_{\theta_{0} a_{(-1)}^{\prime}} n_{a_{(-1)}^{\prime} a_{(-1)}}-\sum_{a_{(0)}} n_{\theta_{0} a_{(0)}}^{P} n_{a_{(0)} a_{(-1)}} \\
- & \sum_{a_{(-2)}} n_{\theta_{0} a_{(-2)}} n_{a_{(-2)} a_{(-1)}}^{P} \\
+ & \sum_{a_{(0)}^{\prime}} n_{\theta_{1} a_{(0)}^{\prime}} m_{a_{(0)}^{\prime} a_{(-1)}}+\sum_{a_{(1)}} n_{\theta_{1} a_{(1)}} m_{a_{(1)} a_{(-1)}}=0
\end{aligned}
$$

Among these terms we can isolate a contribution

$$
\sum_{a_{(-1)}^{\prime}} n_{\theta_{0} a_{(-1)}^{\prime}} n_{a_{(-1)}^{\prime} a_{(-1)}}-\sum_{a_{(0)}} n_{\theta_{0} a_{(0)}}^{P} n_{a_{(0)} a_{(-1)}}-\sum_{a_{(-2)}} n_{\theta_{0} a_{(-2)}} n_{a_{(-2)} a_{(-1)}}^{P}
$$

which is the contribution of the boundary terms in $\mathcal{M}^{P}\left(\theta_{0}, O_{a_{(-1)}}\right)^{*}$ obtained by gluing co-dimension one boundary strata along irreducible critical orbits. The term

$$
\sum_{a_{(-1)}^{\prime}} n_{\theta_{0} a_{(-1)}^{\prime}} n_{a_{(-1)}^{\prime} a_{(-1)}}
$$

counts the contribution of the special gluing

$$
\mathcal{M}\left(\theta_{0}, \theta_{1}\right) \times \mathcal{M}\left(\theta_{1}, a_{(-1)}^{\prime}\right) \times \mathcal{M}\left(a_{(-1)}^{\prime}, a_{(-1)}\right),
$$

where the coefficient

$$
n_{\theta_{0} a_{(-1)}^{\prime}}=\# \bar{s}^{-1}(0)
$$

counts the zeroes of the obstruction section in $\mathcal{M}\left(\theta_{1}, a_{(-1)}^{\prime}\right)$.

According to Theorem 6.22 , the counting of the remaining boundary components for $\mathcal{M}^{P}\left(\theta_{0}, a_{(-1)}\right)$ is given by the gluing

$$
\mathcal{M}\left(\theta_{0}, \theta_{1}\right) \times s^{-1}(0),
$$

with

$$
s^{-1}(0) \subset \mathcal{M}\left(\theta_{1}, a_{(-1)}\right) .
$$

Recall that, in this case, $s^{-1}(0)$ is zero-dimensional.

We claim that the zeroes of this obstruction section are counted precisely by the expression

$$
\sum_{a_{(0)}^{\prime}} n_{\theta_{1} a_{(0)}^{\prime}} m_{a_{(0)}^{\prime} a_{(-1)}}+\sum_{a_{(1)}} n_{\theta_{1} a_{(1)}} m_{a_{(1)} a_{(-1)}}
$$


In fact, we can describe the obstruction bundle as the line bundle

$$
\mathcal{M}\left(\theta_{1}, O_{a_{(-1)}}\right)^{*} \times_{U(1)} \operatorname{Coker}\left(\mathcal{L}_{x}\right)
$$

over $\mathcal{M}\left(\theta_{1}, a_{(-1)}\right)^{*}$, with $x=\left[\mathbb{A}_{0}, 0\right]$ the singular reducible in $\mathcal{M}\left(\theta_{0}, \theta_{1}\right)$, and $\operatorname{Coker}\left(\mathcal{L}_{x}\right)=\mathbb{C}$. With the choice of trivilizations, the zeros of the obstruction section are localized in some compact set in

$$
\begin{gathered}
\bigcup_{a_{(0)}^{\prime}} \mathcal{M}\left(\theta_{1}, a_{(0)}^{\prime}\right) \times \mathcal{M}\left(a_{(0)}^{\prime}, a_{(-1)}\right) \\
\cup \bigcup_{a_{(1)}} \mathcal{M}\left(\theta_{1}, a_{(1)}\right) \times \mathcal{M}\left(a_{(1)}, a_{(-1)}\right) .
\end{gathered}
$$

Thus, counting the zeros of this obstruction section, which gives the relative Euler class of the associated complex line bundle over $\mathcal{M}\left(\theta_{1}, a_{(1)}\right)$, contributes

$$
\begin{aligned}
\# s^{-1}(0)= & \sum_{a_{(0)}^{\prime}} n_{\theta_{1}, a_{(0)}^{\prime}} m_{a_{(0)}^{\prime} a_{(-1)}} \\
+ & \sum_{a_{(1)}} n_{\theta_{1} a_{(1)}} m_{a_{(1)} a_{(-1)}},
\end{aligned}
$$

Then the vanishing condition of (122) follows from \# $\left(\partial \mathcal{M}^{P}\left(\theta_{0}, a_{(-1)}\right)\right)=$ 0 . Thus, we have completed the proof of the identity $1-J I=D H+H D$ on the generator $\Omega^{n} \otimes 1_{\theta_{0}}$.

We proceed to check the remaining identities.

Lemma 6.30. We have the identity $1-J I=D H+H D$ on $\Omega^{n} \otimes 1_{a_{(1)}}$.

Proof. We compute

$$
\begin{aligned}
& (1-J I)\left(\Omega^{n} \otimes 1_{a_{(1)}}\right) \\
= & \Omega^{n} \otimes 1_{a_{(1)}}-J\left(n_{a_{(1)} \theta_{0}} \Omega^{n-1} \otimes 1_{\theta_{1}}+\sum_{a_{(1)}^{\prime}} n_{a_{(1)} a_{(1)}^{\prime}} \Omega^{n-1} \otimes 1_{a_{(1)}^{\prime}}\right) \\
= & \Omega^{n} \otimes 1_{a_{(1)}}-\sum_{\tilde{a}_{(1)}}\left(\sum_{a_{(1)}^{\prime}} n_{a_{(1)} a_{(1)}^{\prime}} n_{a_{(1)}^{\prime} \tilde{a}_{(1)}}+n_{a_{(1)} \theta_{0}} n_{\theta_{1} \tilde{a}_{(1)}}\right) \Omega^{n} \otimes 1_{\tilde{a}_{(1)}} \\
& (D H+H D)\left(\Omega^{n} \otimes 1_{a_{(1)}}\right) \\
= & \left(\sum_{a_{(2)}} n_{a_{(1)} a_{(2)}}^{P} n_{a_{(2)} \tilde{a}_{(1)}}+\sum_{a_{(0)}} n_{a_{(1)} a_{(0)}} n_{a_{(0)} \tilde{a}_{(1)}}^{P}\right) \Omega^{n} \otimes 1_{\tilde{a}_{(1)}} .
\end{aligned}
$$

If we compare the coefficient of each term in $D H+H D-1+J I$, we see that the coefficient of $\Omega^{n} \otimes 1_{a_{(1)}}$ is given by

$$
\sum_{a_{(2)}} n_{a_{(1)} a_{(2)}}^{P} n_{a_{(2)} a_{(1)}}+\sum_{a_{(0)}} n_{a_{(1)} a_{(0)}} n_{a_{(0)} a_{(1)}}^{P}
$$




$$
+\sum_{a_{(1)}^{\prime}} n_{a_{(1)} a_{(1)}^{\prime}} n_{a_{(1)}^{\prime} a_{(1)}}-1+n_{a_{(1)} \theta_{0}} n_{\theta_{1} a_{(1)}}
$$

Notice that the sum

$$
\sum_{a_{(2)}} n_{a_{(1)} a_{(2)}}^{P} n_{a_{(2)} a_{(1)}}+\sum_{a_{(0)}} n_{a_{(1)} a_{(0)}} n_{a_{(0)} a_{(1)}}^{P}+\sum_{a_{(1)}^{\prime}} n_{a_{(1)} a_{(1)}^{\prime}} n_{a_{(1)}^{\prime} a_{(1)}}-1
$$

is the algebraic counting of the boundary points in $\mathcal{M}^{P}\left(a_{(1)}, a_{(1)}\right)^{*}$ that correspond to boundary components of the form

$$
\begin{gathered}
\bigcup_{a_{(2)}} \mathcal{M}^{P}\left(a_{(1)}, a_{(2)}\right) \times \hat{\mathcal{M}}\left(a_{(2)}, a_{(1)}\right) \cup \bigcup_{a_{(0)}} \hat{\mathcal{M}}\left(a_{(1)}, a_{(0)}\right) \times \mathcal{M}^{P}\left(a_{(0)}, a_{(1)}\right) \\
\cup \bigcup_{a_{(1)}^{\prime}} \mathcal{M}\left(a_{(1)}, a_{(1)}^{\prime}\right) \times \mathcal{M}\left(a_{(1)}^{\prime}, a_{(1)}\right) \cup\{-a\} .
\end{gathered}
$$

Similarly, for $\tilde{a}_{(1)} \neq a_{(1)}$, the coefficient of $\Omega^{n} \otimes 1_{\tilde{a}_{(1)}}$ is given by

$$
\begin{aligned}
\sum_{a_{(2)}} n_{a_{(1)} a_{(2)}}^{P} n_{a_{(2)} \tilde{a}_{(1)}}+\sum_{a_{(0)}} n_{a_{(1)} a_{(0)}} n_{a_{(0)} \tilde{a}_{(1)}}^{P} & \\
& +\sum_{a_{(1)}^{\prime}} n_{a_{(1)} a_{(1)}^{\prime}} n_{a_{(1)}^{\prime} \tilde{a}_{(1)}}+n_{a_{(1)} \theta_{0}} n_{\theta_{1} \tilde{a}_{(1)}} .
\end{aligned}
$$

Again, notice that the sum

$$
\sum_{a_{(2)}} n_{a_{(1)} a_{(2)}}^{P} n_{a_{(2)} \tilde{a}_{(1)}}+\sum_{a_{(0)}} n_{a_{(1)} a_{(0)}} n_{a_{(0)} \tilde{a}_{(1)}}+\sum_{a_{(1)}^{\prime}} n_{a_{(1)} a_{(1)}^{\prime}} n_{a_{(1)}^{\prime} \tilde{a}_{(1)}}
$$

is the algebraic counting of the boundary points of $\mathcal{M}^{P}\left(a_{(1)}, \tilde{a}_{(1)}\right)^{*}$ which correspond to boundary components of the form

$$
\begin{aligned}
\bigcup_{a_{(2)}} \mathcal{M}^{P}\left(a_{(1)}, a_{(2)}\right) & \times \hat{\mathcal{M}}\left(a_{(2)}, \tilde{a}_{(1)}\right) \cup \bigcup_{a_{(0)}} \hat{\mathcal{M}}\left(a_{(1)}, a_{(0)}\right) \times \mathcal{M}^{P}\left(a_{(0)}, \tilde{a}_{(1)}\right) \\
& \cup \bigcup_{a_{(1)}^{\prime}} \mathcal{M}\left(a_{(1)}, a_{(1)}^{\prime}\right) \times \mathcal{M}\left(a_{(1)}^{\prime}, \tilde{a}_{(1)}\right) .
\end{aligned}
$$

We only need to prove that the counting of the remaining boundary components of $\mathcal{M}^{P}\left(a_{(1)}, \tilde{a}_{(1)}\right)$ is given by

$$
n_{a_{(1)} \theta_{0}} n_{\theta_{1} \tilde{a}_{(1)}}
$$


which counts the contribution of the zeros of the obstruction section, that is, of those monopoles of $\mathcal{M}^{P}\left(a_{(1)}, \tilde{a}_{(1)}\right)$ which are obtained by gluing the singular reducible $x \in \mathcal{M}\left(\theta_{0}, \theta_{1}\right)$ with

$$
\mathcal{M}\left(a_{(1)}, \theta_{0}\right) \times U(1) \times \mathcal{M}\left(\theta_{1}, \tilde{a}_{(1)}\right) .
$$

Recall that the non-equivariant gluing

$$
\#: \bar{s}^{-1}(0) \subset \mathcal{M}\left(a_{(1)}, \theta_{0}\right) \times U(1) \times \mathcal{M}\left(\theta_{1}, \tilde{a}_{(1)}\right) \rightarrow \mathcal{M}^{P}\left(a_{(1)}, \tilde{a}_{(1)}\right)
$$

corresponds to the equivariant gluing in the framed moduli spaces

$$
\#: \tilde{s}^{-1}(0) \subset \mathcal{M}\left(O_{a_{(1)}}, \theta_{0}\right) \times \mathcal{M}\left(\theta_{1}, O_{\tilde{a}_{(1)}}\right) \rightarrow \mathcal{M}^{P}\left(O_{a_{(1)}}, O_{\tilde{a}_{(1)}}\right) .
$$

The pull-back and push-forward map

$$
\left(e_{\tilde{a}_{(1)}}^{-}\right)_{*}\left(e_{a_{(1)}}^{+}\right)^{*}\left(\eta_{a_{(1)}}\right)
$$

defines the relative Euler characteristic number on the associated line bundle of

This gives

$$
\mathcal{L}_{a_{(1)}, \tilde{a}_{(1)}}=\left(\mathcal{M}\left(O_{a_{(1)}}, \theta_{0}\right) \times \mathcal{M}\left(\theta_{1}, O_{\tilde{a}_{(1)}}\right)\right) \times_{U(1)} \mathbb{C} .
$$

$$
\left(e_{\tilde{a}_{(1)}}^{-}\right)_{*}\left(e_{a_{(1)}}^{+}\right)^{*}\left(\eta_{a_{(1)}}\right)=n_{a_{(1)} \theta_{0}} n_{\theta_{1} \tilde{a}_{(1)}} .
$$

Consider the obstruction bundle

$$
\left(\mathcal{M}\left(O_{a_{(1)}}, \theta_{0}\right) \times \mathcal{M}\left(\theta_{0}, \theta_{1}\right) \times \mathcal{M}\left(\theta_{1}, O_{\tilde{a}_{(1)}}\right)\right) \times_{U(1)} \operatorname{Coker}\left(\mathcal{L}_{x}\right)
$$

over the space

$$
\mathcal{M}\left(a_{(1)}, \theta_{0}\right) \times \mathcal{M}\left(\theta_{1}, \tilde{a}_{(1)}\right) \times U(1)
$$

with $\operatorname{Coker}\left(\mathcal{L}_{x}\right)=\mathbb{C}$, for $x \in \mathcal{M}\left(\theta_{0}, \theta_{1}\right)$ the singular point $x=\left[\mathbb{A}_{0}, 0\right]$. Counting the zeros of the obstruction section

$$
\#\left(\bar{s}^{-1}(0) \cap\left(\mathcal{M}\left(a_{(1)}, \theta_{0}\right) \times \mathcal{M}\left(\theta_{1}, \tilde{a}_{(1)}\right) \times U(1)\right)\right)
$$

gives another computation of the same relative Euler class above, that is,

$$
\begin{aligned}
& \#\left(\bar{s}^{-1}(0) \cap\left(\mathcal{M}\left(a_{(1)}, \theta_{0}\right) \times \mathcal{M}\left(\theta_{1}, \tilde{a}_{(1)}\right) \times U(1)\right)\right) \\
= & n_{a_{(1)} \theta_{0}} n_{\theta_{1} \tilde{a}_{(1)}} .
\end{aligned}
$$

Thus, the components of the boundary of $\mathcal{M}^{P}\left(a_{(1)}, \tilde{a}_{(1)}\right)^{*}$ which come from the gluing with the singular $x \in \mathcal{M}\left(\theta_{0}, \theta_{1}\right)$ contribute a term

$$
n_{a_{(1)} \theta_{0}} n_{\theta_{1} \tilde{a}_{(1)}}
$$


to the counting of the boundary points. This completes the proof of the Lemma.

Finally, we have to check the following.

Lemma 6.31. We have the identity $1-J I=D H+H D$ on $\Omega^{n} \otimes \eta_{a_{(1)}}$.

Proof. Direct calculation of the terms $(1-J I)\left(\Omega^{n} \otimes \eta_{a_{(1)}}\right)$ and $(D H+$ $H D)\left(\Omega^{n} \otimes \eta_{a_{(1)}}\right)$ shows that the conditions required in order to have the same coefficients on all the generators are precisely the conditions already verified in the case of Lemma 6.30.

Notice how clearly this argument of topological invariance breaks down for the non-equivariant Floer homology. The invariance within the same chamber is still verified: in fact, no substantial changes are necessary in that first part of the proof, in order to adapt it to the case of the non-equivariant Floer homology. However, as wee see clearly from the structure of this second part of the proof, the general argument for the proof of Theorem 6.1, for metrics and perturbations in two different chambers, relies essentially on the contribution of the reducible points, in order to construct the chain map $I$ and chain homotopy $H$, as discussed in Definition 6.27, Lemma 6.28, Lemma 6.29, Lemma 6.30, and Lemma 6.31. The example presented at the beginning of Section 6.3 also clarifies why the argument cannot be adapted to the non-equivariant Floer homology.

\section{Wall crossing formula for the Casson-type invariant.}

We want to compare the equivariant Floer homology with the ordinary Floer homology in the cases where the latter is defined, i.e. when $b^{1}(Y)$ is nontrivial [38] or when $Y$ is a homology sphere, [13].

In the case when $b^{1}(Y)$ is non-trivial, we expect to find that the equivariant Floer homology, which is computed by considering framed moduli spaces, is isomorphic to the ordinary Floer homology computed in the unframed space. In fact this is the analogue of the well known result for equivariant cohomology of a finite dimensional manifold, where, if the action of the group is free, then the equivariant cohomology is just the ordinary cohomology of the quotient, $H_{G}^{*}(M ; \mathbb{R}) \cong H^{*}(M / G ; \mathbb{R})$ as $H^{*}(B G ; \mathbb{R})$-modules.

In the case of a homology sphere, instead, we expect to find an exact sequence that connects the equivariant Floer homology with the ordinary 
Floer homology and an extra copy of $\mathbb{R}[\Omega]$ that corresponds to the unique reducible solution that has been removed in the computation of the nonequivariant Floer homology.

Recall that we have the explicit description of the boundary operator in the equivariant Floer complex, as analyzed in Section 5, Proposition 5.3, which gives the formula (72),

$$
\begin{array}{rlc}
\Omega^{n} \otimes 1_{a} & \mapsto & -n_{a b} \Omega^{n} \otimes 1_{b} \\
D: \quad \eta^{n} \otimes \eta_{a} & \mapsto & \left(n_{a b} \Omega^{n} \otimes \eta_{b}\right) \oplus\left(m_{a c} \Omega^{n} \otimes 1_{c}\right) \\
& & \oplus\left(-\Omega^{n-1} \otimes 1_{a}\right)
\end{array}
$$

with the extra components in the case of the generator $\theta$.

\subsection{Comparison with the non-equivariant Floer Homology.}

Now we can define a chain homomorphism that maps the equivariant to the non-equivariant complex.

Let us first work in the case with no reducible solution (i.e. with $b^{1}(Y)>$ 0 ). In this case for each $O_{a}$ that appears in the equivariant complex we have a generator $\mathbb{R} a$ that appears in the non-equivariant complex (coefficients in $\mathbb{R})$.

Now we define the chain map

$$
i_{k}: C_{k, U(1)}(Y) \rightarrow C_{k}(Y),
$$

so that it satisfies $\partial_{k} i_{k}=i_{k-1} D_{k}$. Let $i_{k}$ act on the generators as follows

$$
\begin{gathered}
i_{k}: \bigoplus_{\mu(a)+j=k} \Omega_{j, U(1)}\left(O_{a}\right) \rightarrow \sum_{\mu\left(O_{a}\right)=k} \mathbb{R} a, \\
i_{k}\left(\Omega^{n} \otimes 1_{a}\right)=0
\end{gathered}
$$

for all values of $n$ and $\mu\left(O_{a}\right)$,

$$
i_{k}\left(1 \otimes \eta_{a}\right)=a,
$$

if $\mu\left(O_{a}\right)=k$, and in all other cases

$$
i_{k}\left(\Omega^{n} \otimes \eta_{a}\right)=0
$$

This means that the map $i_{k}$ kills all the generators in degree $k$ that are not the generator of the equivariant homology of some orbit $O_{a}$ of degree $k$.

With this definition it is clear that $i_{*}$ is a chain map. Thus it defines a sub-complex of $C_{*, U(1)}(Y)$ given by $Q_{*}=\operatorname{Ker}\left(i_{*}\right)$ with the restriction of the boundary operator $D$. 
Theorem 7.1. If there are no reducible solutions (i.e., $b^{1}(Y)>0$ ) the map $i_{*}$ defined in (123) induces an isomorphism in cohomology,

$$
H F_{*, U(1)}^{S W}(Y) \cong H F_{*}^{S W}(Y) .
$$

Proof. The complexes $C_{k, U(1)}, Q_{k}$, and $C_{k}$ all have a filtration by index. For $C_{k, U(1)}$ the filtration is given by

$$
C_{k, U(1)}(n)=\bigoplus_{\mu\left(O_{a}\right)+j=k, \mu\left(O_{a}\right) \leq n} \Omega_{j, U(1)}\left(O_{a}\right) .
$$

The complex $Q_{*}$ is written as

$$
Q_{k}=\bigoplus_{\mu(a)+j=k, j \geq 1} \Omega_{j, U(1)}\left(O_{a}\right)
$$

It has a filtration by index of the form

$$
Q_{k}(n)=\bigoplus_{\mu(a)+j=k, j \geq 1, \mu(a) \leq n} \Omega_{j, U(1)}\left(O_{a}\right) .
$$

On the other hand also the non-equivariant complex has a filtration by index of the form

$$
C_{k}(n)=\bigoplus_{\mu(a)=k \leq n} \mathbb{R} a
$$

Thus we can look at the spectral sequences associated to the filtrations and prove that $i$ induces a map of spectral sequences and an isomorphism of the $E^{1}$-terms of the spectral sequences associated to the filtration of $C_{*, U(1)}$ and of $C_{*}$. Thus we get the resulting isomorphism of the $E^{\infty}$ terms, i.e. of the homology of $C_{*, U(1)}$ and of $C_{*}$.

Lemma 7.2. Let $E_{U(1)}, E_{Q}$, and $E$ be the spectral sequences associated to the filtration of the complexes $C_{U(1)}, Q$ and $C$ respectively. The chain map $i_{*}$ induces a map of spectral sequences. Moreover, in the case when $b^{1}(Y)>0$ the map $i_{*}$ induces an isomorphism of the $E^{1}$-terms

$$
E_{k l, U(1)}^{1} \cong E_{k l}^{1} .
$$


Proof. Consider the filtration of $C_{*, U(1)}$. The $E_{k l}^{0}$ terms of the spectral sequence are given by

$$
E_{k l, U(1)}^{0}=C_{k+l, U(1)}(k) / C_{k+l, U(1)}(k-1) .
$$

From (124) we get

$$
E_{k l, U(1)}^{0}=\bigoplus_{\mu\left(O_{a}\right)=k} \Omega_{l, U(1)}\left(O_{a}\right),
$$

and the differentials $E_{k l, U(1)}^{0} \rightarrow E_{k l-1, U(1)}^{0}$ are just given by the differential of the equivariant complex on each fixed orbit $O_{a}$. Thus the $E^{1}$-term of this spectral sequence is given by

$$
\begin{gathered}
E_{k l, U(1)}^{1}=H_{k+l}\left(E_{k *, U(1)}^{0}\right), \\
E_{k l, U(1)}^{1}=\bigoplus_{\mu\left(O_{a}\right)=k} H_{k+l, U(1)}\left(O_{a}\right) .
\end{gathered}
$$

But since the $O_{a}$ are irreducible orbits, with a free $U(1)$ action, the equivariant homology is concentrated in degree zero,

$$
\begin{gathered}
E_{k 0, U(1)}^{1}=\bigoplus_{\mu\left(O_{a}\right)=k} \mathbb{R} a \\
E_{k l, U(1)}^{1}=0
\end{gathered}
$$

for $l \neq 0$.

Now let us consider the filtration of the non-equivariant complex $C_{*}$. From (127) we get

$$
E_{k 0}^{0}=\bigoplus_{\mu\left(O_{a}\right)=k} \mathbb{R} a
$$

and $E_{k l}^{0}=0$ for $l \neq 0$. Thus in this case the only terms that survive in the $E^{1}$ is in degree $l=0$ and is

$$
E_{k 0}^{1}=\bigoplus_{\mu\left(O_{a}\right)=k} \mathbb{R} a
$$

Thus the map $i_{*}$ induces an isomorphism on the $E^{1}$-terms of the two spectral sequences, hence on the $E^{\infty}$-terms, namely on the homology

$$
H_{*}\left(C_{k, U(1)}(Y), D_{k}\right) \stackrel{i}{\cong} H_{*}\left(C_{k}(Y), \partial_{k}\right) .
$$


This completes the proof of Theorem 7.1.

Now let us consider the case when the manifold $Y$ is a rational homology sphere. In this case there are also terms in the equivariant complex that come from the reducible solution $\theta=[\nu, 0]$. We assume that $\theta$ has index zero, $\mu(\theta)=0$.

Theorem 7.3. Let $Y$ be a rational homology sphere. Then there is an exact sequence

$$
\cdots \rightarrow H_{k, U(1)}(\theta) \rightarrow H F_{k, U(1)}^{S W}(Y) \stackrel{i}{\rightarrow} H F_{k}^{S W}(Y) \stackrel{\Delta}{\rightarrow} H_{k-1, U(1)}(\theta) \cdots
$$

In the equivariant complex in degree $k$ we have an extra generator $\Omega^{k} \otimes \theta$. The boundary maps that come from the equivariant complex associated to the degenerate orbit $\nu$ with the trivial action of $U(1)$ are trivial: in fact the equivariant homology of a point is

$$
H_{*, U(1)}(\theta)=\mathbb{R}[\Omega]=H_{*}(B U(1), \mathbb{R}) .
$$

However, there are non-trivial boundary maps that hit the generators $\Omega^{n} \otimes \theta$. These can be described as follows. Suppose $O_{a}$ is the orbit of an irreducible solution with index $\mu\left(O_{a}\right)=1$. Then we have a moduli space $\hat{\mathcal{M}}\left(O_{a}, \theta\right)$ that is 1-dimensional and that fibers over $\theta$ with a 1-dimensional fiber. Thus the pullback-pushforward map acts as

$$
\eta_{a} \mapsto\left(e_{\theta}^{-}\right)_{*}\left(e_{a}^{+}\right)^{*} \eta_{a}=m_{a \theta},
$$

where $m_{a \theta}$ is the integration along the 1-dimensional fiber of the 1-form $\left(e_{a}^{+}\right)^{*} \eta_{a}$. This gives rise to a component of the boundary map of the form

$$
1 \otimes \eta_{a} \mapsto m_{a \theta} 1 \otimes \theta .
$$

Moreover, there is a non-trivial boundary map that comes from the moduli spaces that connect the reducible to generators with the orbits with $\mu\left(O_{a}\right)=$ -2 .

Now the map $i_{k}$ is defined as before with the additional condition that it kills the extra generator $\Omega^{k} \otimes \theta$.

Lemma 7.4. Let $Y$ be a homology sphere. Then the homology of the complex $Q_{*}$ is just the equivariant homology of the point $\theta$,

$$
H_{*}(Q) \cong H_{*, U(1)}(\theta)=\mathbb{R}[\Omega] .
$$


Proof. The complex $Q_{*}$ contains the extra generator $\Omega^{k} \otimes \theta$ in degree $k$ and this generator appears in all levels of the filtration $Q_{k}(n)$ for any $n \geq 0$, since $\theta$ is of degree zero.

Thus if we look at the spectral sequence associated to the filtration of the complex $Q_{*}$ we find

$$
E_{k l, Q}^{0}=Q_{k+l}(k) / Q_{k+l}(k-1),
$$

that is, for $k>0$ and $l \geq 0$,

$$
E_{k l, Q}^{0}=\bigoplus_{\mu\left(O_{b}\right)=k-1} \Omega_{l+1, U(1)}\left(O_{b}\right) .
$$

This complex is clearly acyclic because the differentials are just the equivariant differentials for each orbit and no generator survives in homology because we are counting only $l+1 \geq 1$, hence the terms $1 \otimes \eta_{b}$ are suppressed (in fact they are not in $\operatorname{Ker}\left(i_{*}\right)$ ). On the other hand for $k=0$ we get

$$
E_{0 l, Q}^{0}=\mathbb{R}\left\langle\Omega^{l} \otimes \theta\right\rangle
$$

with trivial differentials, so that the $E^{1}$-terms are

$$
E_{k l, Q}^{1}=H_{k+l, U(1)}(\theta) \text {. }
$$

This means that the homology of the complex $Q_{*}$ is actually $H_{*, U(1)}(\theta)=$ $\mathbb{R}[\Omega]$.

Thus if we consider the long exact sequence induced by the short sequence

$$
0 \rightarrow Q_{k} \rightarrow C_{k, U(1)} \stackrel{i}{\rightarrow} C_{k} \rightarrow 0
$$

we have

$$
\cdots \rightarrow H_{k, U(1)}(\theta) \rightarrow H F_{k, U(1)}^{S W}(Y) \stackrel{i}{\rightarrow} H F_{k}^{S W}(Y) \rightarrow \cdots
$$

This proves Theorem 7.3.

Remark. Notice that, in the case where the spectral flow $S F_{\mathbb{C}}\left(\partial_{\nu_{t}}^{g_{t}}\right)$ is zero, we obtain an isomorphism $H F_{*}^{S W}\left(Y, \mathbf{s}, g_{0}\right) \cong H F_{*}^{S W}\left(Y, \mathbf{s}, g_{1}\right)$ by the five-lemma applied to the exact sequence relating equivariant and nonequivariant Floer homologies.

The connecting homomorphism $\Delta$ in the above long exact sequences is particularly interesting. 
Proposition 7.5. Suppose given a representative $\sum_{a} x_{a} a$ in $H F_{2 k+1}^{S W}(Y)$. We assume that $Y$ is a homology sphere. Then the connecting homomorphism in the long exact sequence is

$$
\triangle_{k}: \quad H F_{2 k+1}^{S W} \rightarrow H_{2 k, U(1)}(\theta)=\mathbb{R} \Omega^{k},
$$

where

$$
\triangle_{k}\left(\sum_{a} x_{a} a\right)=\sum x_{a} m_{a c} m_{c e} \cdots m_{\alpha^{\prime} \alpha} n_{\alpha \theta} \Omega^{k} \otimes \theta .
$$

Here the sum is understood over all the repeated indices, that is over all critical points with indices $\mu\left(O_{a}\right)=2 k+1, \mu\left(O_{c}\right)=2 k-1, \mu\left(O_{\alpha^{\prime}}\right)=3$, $\mu\left(O_{\alpha}\right)=1$.

Proof. The map is defined by the standard diagram chase and by adding boundary terms in order to find a representative of the form (128), as illustrated in the following diagram. Sums over repeated indices are understood.

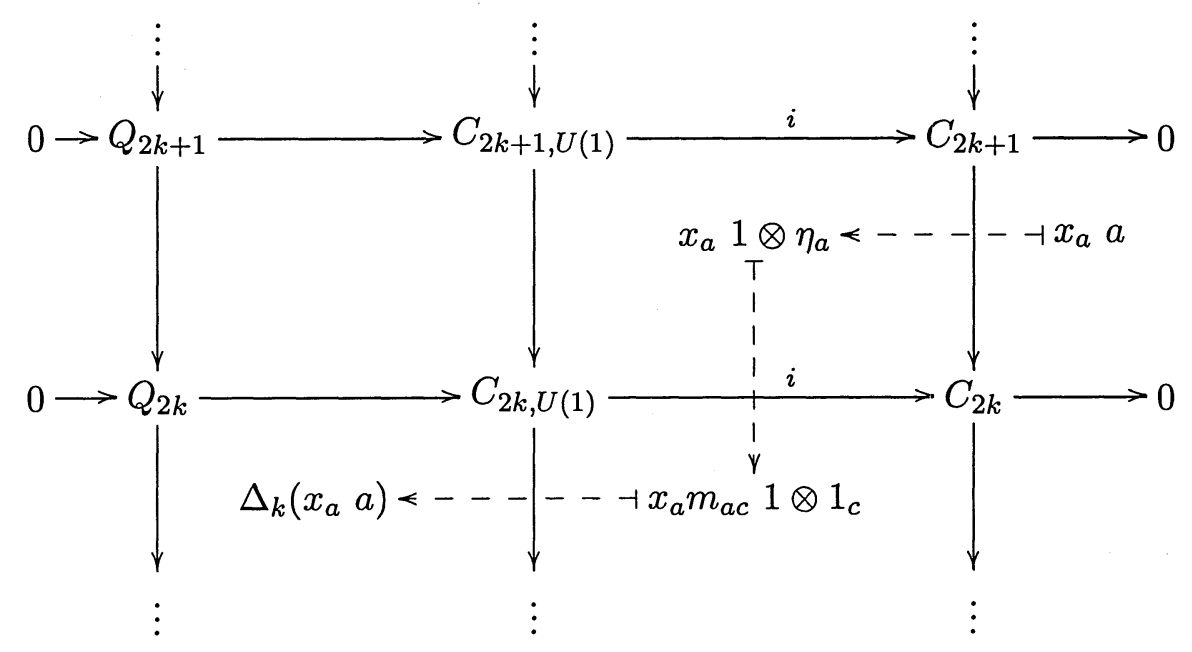

For a cycle $\sum_{a: \mu\left(O_{a}\right)=2 k+1} x_{a} a$ we have $\sum_{a: \mu(a)=2 k+1} x_{a} n_{a b}=0$ for any $b$ with $\mu\left(O_{b}\right)=2 k$. The element $\sum_{a} x_{a} a$ has a preimage $\sum x_{a} 1 \otimes \eta_{a}$ under $i_{2 k+1}$. The image of this element under the equivariant boundary is given by

$$
\sum x_{a} D\left(1 \otimes \eta_{a}\right)=\sum x_{a} n_{a b} 1 \otimes \eta_{b}+\sum x_{a} m_{a c} 1 \otimes 1_{c}
$$


where the first term is zero due to our assumption on $\sum x_{a} a$. The element $\Delta_{k}\left(x_{a} a\right)$ is unchanged if we add to $\sum x_{a} 1 \otimes \eta_{a}$ an element in the kernel of $i_{2 k+1}$. Adding the element $\sum_{a, c} x_{a} m_{a c} \Omega \otimes \eta_{c}$, which is in the kernel of $i$, we get the following diagram

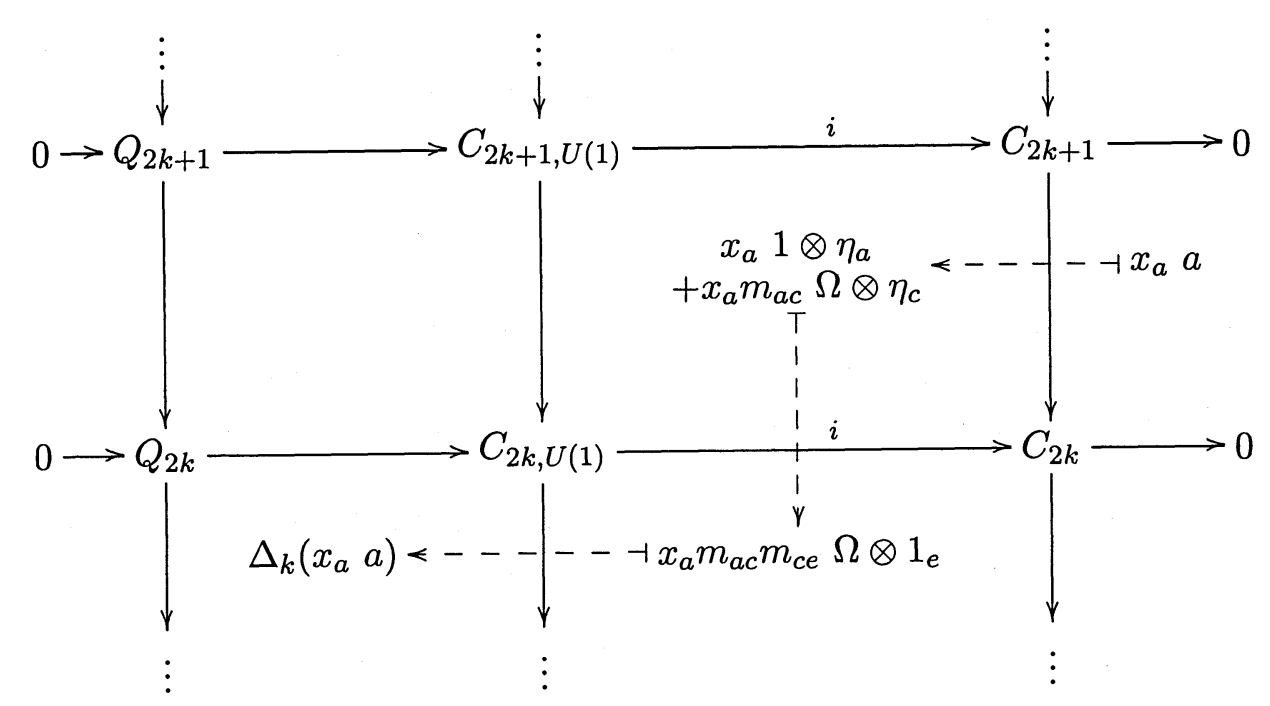

where we have

$$
\begin{gathered}
D\left(x_{a} 1 \otimes \eta_{a}+x_{a} m_{a c} \Omega \otimes \eta_{c}\right)=x_{a} m_{a c} 1 \otimes 1_{c}+ \\
x_{a} m_{a c}\left(-1 \otimes 1_{c}+n_{c d} \Omega \otimes \eta_{d}+m_{c e} \Omega \otimes 1_{e}\right)=x_{a} m_{a c} m_{c e} \Omega \otimes 1_{e}
\end{gathered}
$$

with sums over repeated indices. The last equality follows from the identities $\sum m_{a c} n_{c d}+n_{a b} m_{b d}=0$ and $\sum x_{a} n_{a b} m_{b d}=0$. We can iterate the procedure. In the following step we add a term $\sum x_{a} m_{a c} m_{c e} \Omega^{2} \otimes \eta_{e}$ to the preimage of $\sum x_{a} a$. The corresponding image under the boundary of the equivariant complex is $\sum x_{a} m_{a c} m_{c e} m_{e g} \Omega^{2} \otimes 1_{g}$, where $\mu\left(O_{c}\right)-\mu\left(O_{e}\right)=\mu\left(O_{e}\right)-\mu\left(O_{g}\right)=$ 2. The procedure can be iterated until the reducible point $\theta$ is hit. Contributions from other irreducible critical orbits $O_{p}$ with $\mu\left(O_{p}\right)-\mu(\theta)=0$ are killed in finitely many steps, iterating the same procedure, since the complex is finitely generated and they eventually hit the lowest index critical points. Thus, the resulting image under the connecting homomorphism $\triangle_{k}\left(\sum_{a: \mu a=2 k+1} x_{a} a\right)$ is given by

$$
\sum x_{a} m_{a c} m_{c e} \cdots m_{\alpha^{\prime} \alpha} m_{\alpha \theta} \Omega^{k}
$$


As in the case of instanton Floer theory [62], one expects $\operatorname{Ker}\left(\Delta_{k}\right) \cong$ $\operatorname{Im}\left(i_{2 k+1}\right)$ to be the part of the Floer homology where the relative invariants of four-manifolds with boundary live.

\subsection{Wall-crossing formula: the algebraic picture.}

In this section, we will apply the equivariant Seiberg-Witten-Floer homology theory to study the dependence of the metric for the Casson-type invariant [12], [13] of a rational homology sphere. In order to define the Cassontype invariant, we choose a metric whose ordinary Dirac operator has trivial kernel. The metrics whose ordinary Dirac operator has non-trivial kernel form a chamber structure as proven in Theorem 2.7 and Theorem 2.8. The usual cobordism argument can be adopted to prove that the Casson-type invariant is constant in each chamber. The aim of this section is to get a wall-crossing formula for a path of metrics and perturbations that crosses the wall.

Denote by $\lambda_{S W}(Y, g, \nu)$ the Casson-type invariant for the metric and perturbation $(g, \nu): \lambda_{S W}(Y, g, \nu)$ is the Euler characteristic of the nonequivariant Seiberg-Witten-Floer homology. Recall that this Floer homology [13] is defined by removing the reducible critical point, the trivial solution $\theta=[\nu, 0]$. We have

$$
\lambda_{S W}(Y, g, \nu)=\sum_{k}(-1)^{k} \operatorname{dim} H F_{k}^{S W}(Y, g, \nu) .
$$

In this Section 7.2 we derive the wall crossing formula under the following assumption. Fix metrics and perturbations $\left(g_{0}, \nu_{0}\right),\left(g_{1}, \nu_{1}\right)$ in two different chambers, with the property that there exists an open set of paths $\left(g_{t}, \nu_{t}\right)$ connecting $\left(g_{0}, \nu_{0}\right)$ to $\left(g_{1}, \nu_{1}\right)$ such that $\left(g_{t}, \nu_{t}\right)$ hits a co-dimension one wall only once, transversely.

This situation is certainly verified if the two points $\left(g_{0}, \nu_{0}\right)$ and $\left(g_{1}, \nu_{1}\right)$ are close enough points on either side of a wall $\mathcal{W}_{1}$, in the notation of Theorem 2.8, that is, a wall of metrics and perturbations satisfying $\operatorname{Ker}\left(\partial_{\nu}^{g}\right)=\mathbb{C}$. We also assume that, along the path $\left(g_{t}, \nu_{t}\right)$ we have $S F\left(\partial_{\nu_{t}}^{g_{t}}\right)=2$, that is, that we have $\mu\left(\theta_{0}\right)-\mu\left(\theta_{1}\right)=2$ in the notation used in Section 6 , which determines a global grade shift between the equivariant Floer complexes for $\left(g_{0}, \nu_{0}\right)$ and for $\left(g_{1}, \nu_{1}\right)$.

Using the topological invariance of the equivariant Seiberg-Witten-Floer homology, we obtain the following isomorphism:

$$
H F_{k, U(1)}^{S W}\left(Y, g_{0}, \nu_{0}\right) \cong H F_{k+2, U(1)}^{S W}\left(Y, g_{1}, \nu_{1}\right) .
$$


In fact, the necessary degree shift is computed as follows: if we set $\mu\left(\theta_{0}\right)=0$, hence $\mu\left(\theta_{1}\right)=-2$, and the map $I$ has degree zero, we obtain that the generators $\Omega^{n} \otimes 1_{\theta_{0}}$ and $\Omega^{n+1} \otimes 1_{\theta_{1}}$ have the same degree.

First of all, we express the Casson-type invariant (129) in terms of some alternating sum of the equivariant Seiberg-Witten-Floer homology groups. We consider the Casson-type invariant for metric an perturbation $\left(g_{0}, \nu_{0}\right)$. The exact sequences given in Theorem 7.3, relating the equivariant to the non-equivariant Seiberg-Witten-Floer homology, and the fact that we have $H_{*, U(1)}(\theta)=\mathbb{R}[\Omega]$ give us the following result.

Proposition 7.6. (1) For $k<0$,

$$
H F_{k}^{S W}\left(Y, g_{0}, \nu_{0}\right) \cong H F_{k, U(1)}^{S W}\left(Y, g_{0}, \nu_{0}\right) \text {. }
$$

(2) For $k \geq 0$, we have the following exact sequences

$$
\begin{aligned}
0 \rightarrow & H F_{2 k+1, U(1)}^{S W}\left(Y, g_{0}, \nu_{0}\right) \rightarrow H F_{2 k+1}^{S W}\left(Y, g_{0}, \nu_{0}\right) \rightarrow \mathbb{R} \Omega^{k} \rightarrow \\
& \rightarrow H F_{2 k, U(1)}^{S W}\left(Y, g_{0}, \nu_{0}\right) \rightarrow H F_{2 k}^{S W}\left(Y, g_{0}, \nu_{0}\right) \rightarrow 0 .
\end{aligned}
$$

Thus the dimensions are related by

$$
\operatorname{dim} H F_{2 k}^{S W}-\operatorname{dim} H F_{2 k+1}^{S W}=\operatorname{dim} H F_{2 k, U(1)}^{S W}-\operatorname{dim} H F_{2 k+1, U(1)}^{S W}-1 .
$$

This gives the wall-crossing formula for the Casson-type invariant.

Theorem 7.7. Suppose given two metrics and perturbations $\left(g_{0}, \nu_{0}\right)$ and $\left(g_{1}, \nu_{1}\right)$ in two different chambers and a generic path $\left(g_{t}, \nu_{t}\right)$ that connects them and that crosses the wall $\mathcal{W}$ once. Assume that the crossing happens at a generic point of $\mathcal{W}$, so that the relative Morse index with respect to the reducible solution decreases by 2 across the wall, i.e., that we have $\mu\left(\theta_{0}\right)-$ $\mu\left(\theta_{1}\right)=2$. Then the Casson-type invariant changes by

$$
\lambda_{S W}\left(Y, g_{1}, \nu_{1}\right)=\lambda_{S W}\left(Y, g_{0}, \nu_{0}\right)-1 .
$$

Proof. We can assume that we are in one of the following two cases for the non-equivariant Floer homology group.

Case 1. There exists an integer $N$ such that $H F_{p}^{S W}\left(Y, g_{0}, \nu_{0}\right)=0$ for all $p \geq 2 N$ but $H F_{2 N-1}^{S W}\left(Y, g_{0}, \nu_{0}\right) \neq 0$. 
Case 2. Here exists an integer $N$ such that $H F_{p}^{S W}\left(Y, g_{0}, \nu_{0}\right)=0$ for all $p \geq 2 N+1$ but $H F_{2 N}^{S W}\left(Y, g_{0}, \nu_{0}\right) \neq 0$.

For case 1 , we can assume $N \geq 0$, otherwise the non-equivariant Floer homology groups are isomorphic to the equivariant Floer homology groups by Proposition 7.6. Therefore, from the exact sequences in Proposition 7.6,
we get

- $H F_{p, U(1)}^{S W}\left(Y, g_{0}, \nu_{0}\right)= \begin{cases}0 & p>2 N, \mathrm{p} \text { is odd } \\ \mathbb{R} \Omega^{m} & p=2 m \geq 2 N\end{cases}$

$$
\begin{aligned}
& \operatorname{dim} H F_{2 N-2}^{S W}\left(Y, g_{0}, \nu_{0}\right)-\operatorname{dim} H F_{2 N-1}^{S W}\left(Y, g_{0}, \nu_{0}\right) \\
= & \operatorname{dim} H F_{2 N-2, U(1)}^{S W}\left(Y, g_{0}, \nu_{0}\right)-\operatorname{dim} H F_{2 N-1, U(1)}^{S W}\left(Y, g_{0}, \nu_{0}\right)-1 \\
& \operatorname{dim} H F_{2 N-4}^{S W}\left(Y, g_{0}\right)-\operatorname{dim} H F_{2 N-3}^{S W}\left(Y, g_{0}, \nu_{0}\right) \\
= & \operatorname{dim} H F_{2 N-4, U(1)}^{S W}\left(Y, g_{0}, \nu_{0}\right)-\operatorname{dim} H F_{2 N-3, U(1)}^{S W}\left(Y, g_{0}, \nu_{0}\right)-1 \\
& \cdots \\
& \operatorname{dim} H F_{0}^{S W}\left(Y, g_{0}, \nu_{0}\right)-\operatorname{dim} H F_{1}^{S W}\left(Y, g_{0}, \nu_{0}\right) \\
= & \operatorname{dim} H F_{0, U(1)}^{S W}\left(Y, g_{0}, \nu_{0}\right)-\operatorname{dim} H F_{1, U(1)}^{S W}\left(Y, g_{0}, \nu_{0}\right)-1 .
\end{aligned}
$$

- For $k<0, H F_{k}^{S W}\left(Y, g_{0}, \nu_{0}\right)=H F_{k, U(1)}^{S W}\left(Y, g_{0}, \nu_{0}\right)$.

Given the above information, we can calculate the Casson-type invariant for the metric and perturbation $\left(g_{0}, \nu_{0}\right)$ as,

$$
\begin{aligned}
\lambda_{S W}\left(Y, g_{0}, \nu_{0}\right)= & \sum_{k}(-1)^{k} \operatorname{dim} H F_{k}^{S W}\left(Y, g_{0}, \nu_{0}\right) \\
= & \sum_{k<N}\left(\operatorname{dim} H F_{2 k, U(1)}^{S W}\left(Y, g_{0}, \nu_{0}\right)\right. \\
& \left.-\operatorname{dim} H F_{2 k+1, U(1)}^{S W}\left(Y, g_{0}, \nu_{0}\right)\right)-N .
\end{aligned}
$$
have

From the isomorphism $H F_{k, U(1)}^{S W}\left(Y, g_{1}, \nu_{1}\right)=H F_{k-2, U(1)}^{S W}\left(Y, g_{0}, \nu_{0}\right)$, we

$$
H F_{p, U(1)}^{S W}\left(Y, g_{1}, \nu_{1}\right)= \begin{cases}0 & p>2 N+2, \mathrm{p} \text { is odd } \\ \mathbb{R} \Omega^{m} & p=2 m \geq 2 N+2 .\end{cases}
$$

If we apply the above isomorphisms to the exact sequences in Proposition 7.6, we can see that the Casson-type invariant for metric and perturbation 
$\left(g_{1}, \nu_{1}\right)$ can be rewritten as

$$
\begin{aligned}
\lambda_{S W}\left(Y, g_{1}, \nu_{1}\right)= & \sum_{k}(-1)^{k} \operatorname{dim} H F_{k}^{S W}\left(Y, g_{1}, \nu_{1}\right) \\
= & \sum_{k<N+1}\left(\operatorname{dim} H F_{2 k, U(1)}^{S W}\left(Y, g_{1}, \nu_{1}\right)\right. \\
& \left.-\operatorname{dim} H F_{2 k+1, U(1)}^{S W}\left(Y, g_{1}, \nu_{1}\right)\right)-(N+1) \\
= & \lambda_{S W}\left(Y, g_{0}, \nu_{0}\right)-1
\end{aligned}
$$

For case 2 , similarly, we have

$$
\text { - } H F_{p, U(1)}^{S W}\left(Y, g_{0}, \nu_{0}\right)= \begin{cases}0 & p>2 N, \mathrm{p} \text { is odd } \\ \mathbb{R} \Omega^{m} & p=2 m \geq 2 N+2\end{cases}
$$

$$
\begin{aligned}
& \operatorname{dim} H F_{2 N}^{S W}\left(Y, g_{0}, \nu_{0}\right)=\operatorname{dim} H F_{2 N, U(1)}^{S W}\left(Y, g_{0}, \nu_{0}\right)-1 \\
& \operatorname{dim} H F_{2 N-2}^{S W}\left(Y, g_{0}, \nu_{0}\right)-\operatorname{dim} H F_{2 N-1}^{S W}\left(Y, g_{0}, \nu_{0}\right) \\
= & \operatorname{dim} H F_{2 N-2, U(1)}^{S W}\left(Y, g_{0}, \nu_{0}\right)-\operatorname{dim} H F_{2 N-1, U(1)}^{S W}\left(Y, g_{0}, \nu_{0}\right)-1 \\
& \operatorname{dim} H F_{2 N-4}^{S W}\left(Y, g_{0}, \nu_{0}\right)-\operatorname{dim} H F_{2 N-3}^{S W}\left(Y, g_{0}, \nu_{0}\right) \\
= & \operatorname{dim} H F_{2 N-4, U(1)}^{S W}\left(Y, g_{0}, \nu_{0}\right)-\operatorname{dim} H F_{2 N-3, U(1)}^{S W}\left(Y, g_{0}, \nu_{0}\right)-1 \\
& \cdots \\
& \operatorname{dim} H F_{0}^{S W}\left(Y, g_{0}, \nu_{0}\right)-\operatorname{dim} H F_{1}^{S W}\left(Y, g_{0}, \nu_{0}\right) \\
= & \operatorname{dim} H F_{0, U(1)}^{S W}\left(Y, g_{0}, \nu_{0}\right)-\operatorname{dim} H F_{1, U(1)}^{S W}\left(Y, g_{0}, \nu_{0}\right)-1
\end{aligned}
$$

- For $k<0, H F_{k}^{S W}\left(Y, g_{0}, \nu_{0}\right)=H F_{k, U(1)}^{S W}\left(Y, g_{0}, \nu_{0}\right)$.

Then the Casson-type invariant for $\left(g_{0}, \nu_{0}\right)$ is

$$
\begin{aligned}
\lambda_{S W}\left(Y, g_{0}, \nu_{0}\right)= & \sum_{k}(-1)^{k} \operatorname{dim} H F_{k}^{S W}\left(Y, g_{0}, \nu_{0}\right) \\
= & \sum_{k<N}\left(\operatorname{dim} H F_{2 k, U(1)}^{S W}\left(Y, g_{0}, \nu_{0}\right)\right. \\
& \left.-\operatorname{dim} H F_{2 k+1, U(1)}^{S W}\left(Y, g_{0}, \nu_{0}\right)\right)-N \\
& +\operatorname{dim} H F_{2 N, U(1)}^{S W}\left(Y, g_{0}, \nu_{0}\right)-1 .
\end{aligned}
$$

From the isomorphism $H F_{k, U(1)}^{S W}\left(Y, g_{1}, \nu_{1}\right)=H F_{k-2, U(1)}^{S W}\left(Y, g_{0}, \nu_{0}\right)$, we know that

$$
H F_{p, U(1)}^{S W}\left(Y, g_{1}, \nu_{1}\right)= \begin{cases}0 & p>2 N+2, \mathrm{p} \text { is odd } \\ \mathbb{R} \Omega^{m} & p=2 m \geq 2 N+2 .\end{cases}
$$


If we apply the above isomorphisms to the exact sequences in Proposition 7.6 again, we can see that the Casson-type invariant for metric and perturbation $\left(g_{1}, \nu_{1}\right)$ is

$$
\begin{aligned}
& \lambda_{S W}\left(Y, g_{1}, \nu_{1}\right) \\
= & \sum_{k}(-1)^{k} \operatorname{dim} H F_{k}^{S W}\left(Y, g_{1}, \nu_{1}\right) \\
= & \sum_{k<N+1}\left(\operatorname{dim} H F_{2 k, U(1)}^{S W}\left(Y, g_{1}, \nu_{1}\right)-\operatorname{dim} H F_{2 k+1, U(1)}^{S W}\left(Y, g_{1}, \nu_{1}\right)\right) \\
& -(N+1)+\operatorname{dim} H F_{2 N+2 . U(1)}^{S W}\left(Y, g_{1}, \nu_{1}\right)-1 \\
= & \lambda_{S W}\left(Y, g_{0}, \nu_{0}\right)-1
\end{aligned}
$$

Thus, we have proven the wall-crossing formula:

$$
\lambda_{S W}\left(Y, g_{1}, \nu_{1}\right)=\lambda_{S W}\left(Y, g_{0}, \nu_{0}\right)-1 .
$$

Now we are interested in generalizing the argument of Theorem 7.7 to the case of a path $\left(g_{t}, \nu_{t}\right)$ that crosses the wall structure $\mathcal{W}$ at a point which lies in a stratum $\mathcal{W}_{n}$ of higher codimension.

If we know that a stratum $\mathcal{W}_{n}$ of metrics and perturbations satisfying $\operatorname{Ker}\left(\partial_{\nu}^{g}\right)=\mathbb{C}^{n}$ is obtained as the transverse intersection of $n$ strata $\mathcal{W}_{1}^{i_{1}} \cap$ $\cdots \cap \mathcal{W}_{1}^{i_{n}}$, where every $\mathcal{W}_{1}^{i_{k}}$ consists of metrics with $\operatorname{Ker}\left(\partial_{\nu}^{g}\right)=\mathbb{C}$, then a path $\left(g_{t}, \nu_{t}\right)$ that crosses $\mathcal{W}$ at a point in $\mathcal{W}_{n}$ can be deformed to a path that crosses each $\mathcal{W}_{1}^{i_{k}}$ once transversely. In this case, the wall crossing formula simply follows by applying repeatedly Theorem 7.7. However, we do not really need the assumption on the structure of $\mathcal{W}$ near a stratum of higher codimension. In fact, it is enough to know that the complex spectral flow $S F_{\mathbb{C}}\left(\partial_{\nu_{t}}^{g_{t}}\right)=\frac{1}{2} S F\left(\partial_{\nu_{t}}^{g_{t}}\right)$ is equal to $\pm n$ along the path $\left(g_{t}, \nu_{t}\right)$ that crosses a point on $\mathcal{W}_{n}$. In that case, we can follow the same argument in the proof of Theorem 7.7, but starting with a grade shift of $2 n$ between the equivariant Floer complexes for $\left(g_{0}, \nu_{0}\right)$ and $\left(g_{1}, \nu_{1}\right)$. We obtain the following result.

Proposition 7.8. Let $\left(g_{0}, \nu_{0}\right)$ and $\left(g_{1}, \nu_{1}\right)$ be two metrics and perturbations in two different chambers. Suppose given a path $\left(g_{t}, \nu_{t}\right)$ joining them that crosses the wall $\mathcal{W}$ once transversely at a point of a stratum $\mathcal{W}_{n}$ of codimension $2 n-1$. The relative Morse index with respect to the reducible solution decreases by $2 n$ across the wall, that is, that we have $\mu\left(\theta_{0}\right)-\mu\left(\theta_{1}\right)=2 n$. Then the Casson-type invariant changes by

$$
\lambda_{S W}\left(Y, g_{1}, \nu_{1}\right)=\lambda_{S W}\left(Y, g_{0}, \nu_{0}\right)-n .
$$


Since the argument of Theorem 7.7 depends only on the counting of the grade shift between the Floer complexes for $\left(g_{0}, \nu_{0}\right)$ and $\left(g_{1}, \nu_{1}\right)$ given by the spectral flow $S F\left(\partial_{\nu_{t}}^{g_{t}}\right)$, together with the proof of topological invariance of the equivariant Floer homology (up to this grade shift), we can formulate the result under these more general hypothesis.

Proposition 7.9. Let $\left(g_{0}, \nu_{0}\right)$ and $\left(g_{1}, \nu_{1}\right)$ be two metrics and perturbations in two different chambers. Suppose given a path $\left(g_{t}, \nu_{t}\right)$ joining them that crosses the wall $\mathcal{W}$ transversely in finitely many points. Then the Casson invariant changes by

$$
\lambda_{S W}\left(Y, g_{1}, \nu_{1}\right)=\lambda_{S W}\left(Y, g_{0}, \nu_{0}\right)-S F_{\mathbb{C}}\left(\partial_{\nu_{t}}^{g_{t}}\right),
$$

where $S F_{\mathbb{C}}\left(\partial_{\nu_{t}}^{g_{t}}\right)=\frac{1}{2} S F\left(\partial_{\nu_{t}}^{g_{t}}\right)$ is the complex spectral flow of the Dirac operator along the path of reducible solutions $\left[\nu_{t}, 0\right]$.

Proof. If the spectral flow along the path $\left(g_{t}, \nu_{t}\right)$ is given by $S F\left(\partial_{\nu_{t}}^{g_{t}}\right)$, the topological invariance of the equivariant Floer homology gives

$$
H F_{k, U(1)}^{S W}\left(Y, g_{0}, \nu_{0}\right) \cong H F_{k+S F\left(\partial_{\nu_{t}}^{g_{t}}\right), U(1)}^{S W}\left(Y, g_{1}, \nu_{1}\right) \text {. }
$$

We can then follow the steps of the proof of Theorem 7.7 and compare the ranks of the Floer groups and the counting of the Euler characteristic. This can be done by induction on $\left|S F\left(\partial_{\nu_{t}}^{g_{t}}\right)\right|$. The result is the formula (130).

The wall crossing formula in the case of $J$-invariant perturbations constructed by W. Chen [14] can also be derived with the same method. This gives rise to the following wall crossing result.

Corollary 7.10. Consider the invariant $\lambda_{S W}(Y, g, f)$ where $f$ is the $J$ invariant perturbation of [14] Prop. 2.6.

Given two metrics and perturbations $\left(g_{0}, f_{0}\right)$ and $\left(g_{1}, f_{1}\right)$ in two different chambers and a generic path $\left(g_{t}, f_{t}\right)$ that crosses the wall once with $\operatorname{Ker}\left(\partial_{f}^{g}\right)=\mathbb{H}$. The relative Morse index with respect to the reducible solution decreases by 4 across the wall, namely, we have $\mu\left(\theta_{0}\right)-\mu\left(\theta_{1}\right)=4$. Then the

Casson-type invariant changes by

$$
\lambda_{S W}\left(Y, g_{1}, f_{1}\right)=\lambda_{S W}\left(Y, g_{0}, f_{0}\right)-2 .
$$


Proof. Again the main issue is the change of grading of the equivariant Floer homology induced by the spectral flow of the Dirac operator along the path $\left(g_{t}, f_{t}\right)$. This time, since we are using $J$-invariant perturbations, the Dirac operator $\partial_{f}^{g}$ is quaternion linear, hence, for the chosen path the spectral flow satisfies $S F\left(\partial_{f_{t}}^{g_{t}}\right)=4$. This implies that there is a degree shift

$$
H F_{k, U(1)}^{S W}\left(Y, g_{0}, f_{0}\right) \cong H F_{k+4, U(1)}^{S W}\left(Y, g_{1}, f_{1}\right) .
$$

By applying the previous Proposition 7.9, we obtain the result.

\subsection{Wall-crossing formula: the geometric picture.}

In this section we re-derive, in a more geometric way, the wall crossing formula for a homology three-sphere $Y$ that we proved algebraically in the previous section. We analyze the local structure of the parameterized moduli space. A geometric proof of the wall-crossing formula has been also worked out by [35].

Let $\mathcal{M}^{*}(g, \nu)$ denote the irreducible part of the moduli space $\mathcal{M}$ for the metric and perturbation $(g, \nu)$. Given a family of metrics and perturbation $\left(g_{t}, \nu_{t}\right)$ with $(t \in[-1,1])$, the moduli spaces $\mathcal{M}^{*}\left(g_{-1}, \nu_{-1}\right)$ and $\mathcal{M}^{*}\left(g_{1}, \nu_{1}\right)$ are cobordant as long as the path $\left(g_{t}, \nu_{t}\right)$ does not cross the wall, that is the co-dimension one subspace $W$ in the space of metrics and perturbations

$$
W=\left\{(g, \nu) \mid \operatorname{Ker}\left(\partial_{\nu}^{g}\right) \neq 0\right\} .
$$

Suppose the path $\left(g_{t}, \nu_{t}\right)$ crosses the wall $W$ just once at $t=0$. Generically, $\operatorname{Ker}\left(\partial_{\nu_{0}}^{g_{0}}\right) \cong \mathbb{C}$. We want to analyze the local structure of the parameterized moduli space

$$
\overline{\mathcal{M}}=\left\{\mathcal{M}\left(g_{t}, \nu_{t}\right) \times\{t\} \mid t \in[-1,1]\right\}
$$

at the reducible point $\vartheta_{0}=\left(\theta_{0}, 0\right)$, where $\theta_{0}=\left[\nu_{0}, 0\right]$ is the class of the reducible solution of (15) with the metric and perturbation $\left(g_{0}, \nu_{0}\right)$. There is a family of reducibles $\vartheta_{t}$ in $\overline{\mathcal{M}}$. Let $\overline{\mathcal{M}}^{*}$ be the irreducible set in $\overline{\mathcal{M}}, \mathcal{U}$ be a sufficiently small neighborhood of $\theta_{0}$ in $\overline{\mathcal{M}}$, and $\mathcal{U}^{*}$ be the irreducible part of $\mathcal{U}$.

We construct a bundle over neighborhood of $\vartheta_{0}$ in $\mathcal{A} \times[-1,1]$, together with a section $\varsigma$ such that

$$
\mathcal{U}^{*}=\left(\varsigma^{-1}(0)-\left\{\left(\vartheta_{t}, t\right)\right\}\right) / \mathcal{G}
$$


Lemma 7.11. (1) The slice of the $\mathcal{G} / U(1)$-action at a point $\left(A_{0}, 0\right)$ is $V_{\left(A_{0}, 0\right)}=\operatorname{Ker}\left(d^{*}\right) \times \Gamma_{L^{2}}(S)$,

(2) The slice of the $\mathcal{G} / U(1)$-action at a point $(A, \psi)$ is

$$
V_{(A, \psi)}=\left\{(\alpha, \phi) \mid d^{*}(\alpha)-2 i \operatorname{Im}\langle\phi, \psi\rangle \text { is a constant function on } Y .\right\}
$$

(3) For $(A, \psi)$ close to $\left(A_{0}, 0\right)$ there is an isomorphism

$$
\lambda_{(A, \psi)}: \quad V_{(A, \psi)} \rightarrow V_{\left(A_{0}, 0\right)}
$$

Proof. Properties (1) and (2) follow by direct computation. For (3), choose $(\alpha, \phi)$ in $V_{(A, \psi)}$ and define $\lambda_{(A, \psi)}(\alpha, \phi)$ to be

$$
\left(\alpha-2 d \xi_{(\alpha, \phi)}, \xi_{(\alpha, \phi)} \psi+\phi\right)
$$

where $\xi_{(\alpha, \phi)}$ is the unique solution of the following equations:

$$
\left\{\begin{aligned}
2 d^{*} d \xi_{(\alpha, \phi)} & =d^{*} \alpha \\
\int_{Y} \xi_{(\alpha, \phi)} d v & =0
\end{aligned}\right.
$$

Direct computation shows that $\lambda_{(A, \psi)}$ is an isomorphism.

The above Lemma shows that we obtain a locally trivial vector bundle $V$ over the space of connections and sections $\mathcal{A}$ endowed with a $U(1)$-action.

Define the section $\varsigma$

$$
\varsigma: \mathcal{A} \times[-1,1] \rightarrow V
$$

to be

$$
\varsigma(A, \psi, t)=\lambda_{(A, \psi)}\left(*_{g_{t}}\left(F_{A}-d \nu_{t}\right)-\sigma(\psi, \psi), \partial_{A} \psi\right) .
$$

Near $\theta_{0}$, we know that $\mathcal{U}=\varsigma^{-1}(0) / \mathcal{G}$. Therefore, the local structure of $\mathcal{U}^{*}$ at $\theta_{0}$ is given by the Kuranishi model of $\varsigma^{-1}(0) / \mathcal{G}$ at $\theta_{0}$.

Suppose $\left(A_{t}, \psi_{t}\right)$ is an element in $\mathcal{U}^{*}$. Consider a formal expansion at $\vartheta_{0}$ of the form

$$
\begin{gathered}
A_{t}=\nu_{t}+t \alpha_{1}+t^{2} \alpha_{2}+\cdots \\
\psi_{t}=t \psi_{1}+t^{2} \psi_{2}+\cdots
\end{gathered}
$$


The section $\varsigma$ is approximated by

$$
\begin{gathered}
*_{g_{0}} d\left(\nu_{t}+t \alpha_{1}+t^{2} \alpha_{2}+\cdots\right)-*_{g_{0}} d \nu_{t} \\
\quad-\sigma\left(t \psi_{1}+t^{2} \psi_{2}+\cdots, t \psi_{1}+t^{2} \psi_{2}+\cdots\right) \\
\partial_{\nu_{t}}^{g_{0}}\left(t \psi_{1}+t^{2} \psi_{2}+\cdots\right)+\left(t \alpha_{1}+t^{2} \alpha_{2}+\cdots\right) \cdot\left(t \psi_{1}+t^{2} \psi_{2}+\cdots\right)
\end{gathered}
$$

where we are perturbing in a neighborhood of the wall $W$ just by changing the perturbation and fixing the metric $g_{0}$.

The zero set of the section therefore determines the conditions $* d \alpha_{1}=0$ and $d^{*} \alpha_{1}=0$, which imply $\alpha_{1}=0$ on a homology sphere. Moreover, we have $d^{*} \alpha_{2}=0$ and

$$
* d \alpha_{2}=\sigma\left(\psi_{1}, \psi_{1}\right) .
$$

On the kernel of $d^{*}$ the operator $* d$ is invertible, hence we have

$$
\alpha_{2}=(* d)^{-1} \sigma\left(\psi_{1}, \psi_{1}\right) .
$$

The Kuranishi model near $\vartheta_{0}$ is given by a $U(1)$-equivariant map

$$
\mathcal{S}: \mathbb{R} \times \operatorname{Ker}\left(\partial_{\nu_{0}}^{g_{0}}\right) \rightarrow \operatorname{CoKer}\left(\partial_{\nu_{0}}^{g_{0}}\right),
$$

where $U(1)$ acts on $\operatorname{Ker}\left(\partial_{\nu_{0}}^{g_{0}}\right) \cong \operatorname{CoKer}\left(\partial_{\nu_{0}}^{g_{0}}\right) \cong \mathbb{C}$ by the natural multiplication on $\mathbb{C}$.

There exists a sufficiently small $\delta>0$ such that, for $t \in[-\delta, \delta]$, we have that $\partial_{\nu_{t}}^{g_{0}}$ has exactly one small eigenvalue $\lambda(t)$ with eigenvector $\phi_{t}$ and with $\lambda(0)=0$, that is

$$
\partial_{\nu_{t}}^{g_{0}} \phi_{t}=\lambda(t) \phi_{t}
$$

This implies that, if $\lambda^{\prime}(0)>0$, then the spectral flow of $\partial_{\nu_{t}}^{g_{0}}$ for $(t \in$ $[-1,1])$ is 1 and, if $\lambda^{\prime}(0)<0$, the spectral flow of $\partial_{\nu_{t}}^{g_{0}}$ for $(t \in[-1,1])$ is -1 .

The map $\mathcal{S}$ is given by

$$
\begin{gathered}
\mathcal{S}: \mathbb{R} \times \mathbb{C} \rightarrow \mathbb{C}, \\
\mathcal{S}(t, w \phi)=\Pi_{\operatorname{Ker}\left(\partial_{\nu_{0}}^{g_{0}}\right)}\left(\partial_{A_{t}} w \phi\right) .
\end{gathered}
$$

Here we assume that $\phi$ is a spinor in $\operatorname{Ker}\left(\partial_{\nu_{0}}^{g_{0}}\right)$ with $\|\phi\|=1$, so that $\operatorname{Ker}\left(\partial_{\nu_{0}}^{g_{0}}\right) \cong \mathbb{C} \phi$. Consider the expression

$$
\left\langle\partial_{\nu_{t}}^{g_{0}} \phi, \phi\right\rangle=z(t) .
$$

Notice that we have $z^{\prime}(0)=\lambda^{\prime}(0)$, in fact, we write formally $\lambda(t) \sim t \lambda^{\prime}(0)$, $\phi_{t} \sim \phi+t \phi_{1}$ and the Dirac operator $\partial_{\nu_{t}}^{g_{0}} \sim \partial_{\nu_{0}}^{g_{0}}+t C$, where $\nu_{t} \sim \nu_{0}+t \nu_{1}$ and 
$C$ acts as Clifford multiplication by $\nu_{1}$. We can write the first order term in the relation $\partial_{\nu_{t}}^{g_{0}} \phi_{t}=\lambda(t) \phi_{t}$ as

$$
t\left\langle\partial_{\nu_{0}}^{g_{0}} \phi_{1}, \phi\right\rangle+t\langle C \phi, \phi\rangle=t \lambda^{\prime}(0)+\lambda(0) .
$$

Here the term $\left\langle\partial_{\nu_{0}}^{g_{0}} \phi_{1}, \phi\right\rangle=\left\langle\phi_{1}, \partial_{\nu_{0}}^{g_{0}} \phi\right\rangle$ vanishes, and also $\lambda(0)=0$. Thus, we have the relation

$$
\langle C \phi, \phi\rangle=\lambda^{\prime}(0) .
$$

On the other hand, we have

$$
\langle C \phi, \phi\rangle=z^{\prime}(0)
$$

from the expansion of $\partial_{\nu_{t}}^{g_{0}} \phi=z(t) \phi$.

Thus the map $\mathcal{S}$ can be rewritten as

$$
\begin{aligned}
\mathcal{S}(t, w \phi) & =z(t) w \phi+t^{2}\left\langle\alpha_{2} \phi, \phi\right\rangle w \phi+O\left(t^{3}\right) \\
& =w \phi\left(z(t)+t^{2} r^{2}\left\langle(* d)^{-1} \sigma(\phi, \phi), \sigma(\phi, \phi)\right\rangle\right)+O\left(t^{3}\right) .
\end{aligned}
$$

Here we use the fact that the first order term of the Dirac equation gives $\partial_{\nu_{0}}^{g_{0}} \psi_{1}=0$, therefore $\psi_{1}=r e^{i \theta} \phi$ and $\sigma\left(\psi_{1}, \psi_{1}\right)=r^{2} \sigma(\phi, \phi)$.

The term

$$
\gamma\left(Y, g_{0}, \nu_{0}\right)=\left\langle(* d)^{-1} \sigma(\phi, \phi), \sigma(\phi, \phi)\right\rangle
$$

is a constant that only depends on the manifold and on $\theta_{0}$. An inductive argument shows that, if $\gamma\left(Y, g_{0}, \nu_{0}\right)$ vanishes, then all the forms $\alpha_{i}$ in the formal expansion of $A_{t}$ must also vanish identically. Thus, we can assume that $\gamma\left(Y, g_{0}, \nu_{0}\right) \neq 0$.

Notice that we have

$$
\mathbb{R} \times\left(\operatorname{Ker}\left(\partial_{\nu_{0}}^{g_{0}}\right)-\{0\}\right) / U(1)=\mathbb{R} \times \mathbb{R}^{+} .
$$

The irreducible part of $\varsigma^{-1}(0) / \mathcal{G}$ is tangent to $\{0\} \times \mathbb{R}^{+}$as $t$ approaches 0 , as we see in the following.

The difference $\lambda$ between the Casson-type invariant at $t= \pm \delta$ can be evaluated by counting the number (with sign) of oriented lines in $\varsigma^{-1}(0) / \mathcal{G}$, with $t \in[-\delta, \delta]$, that are tangent to $\{0\} \times \mathbb{R}^{+} \times\{0\}$. Here we identify $\mathcal{U}^{*}$ with the set $\left(\mathcal{S}^{-1}(0)-\{w=0\}\right) / U(1)$. The sign of the wall crossing term is determined by the section $\mathcal{S}$, as follows. The zero set $\left(\mathcal{S}^{-1}(0)-\{w=\right.$ $0\}) / U(1)$ is given by the condition

$$
t=-\frac{\lambda^{\prime}(0)}{r^{2} \gamma\left(Y, g_{0}, \nu_{0}\right)} .
$$


Thus, we have one line in $\mathcal{U}^{*}$ which is counted with the orientation determined by the sign of $-\gamma\left(Y, g_{0}, \nu_{0}\right)$ and the spectral flow. Suppose that we have $\lambda^{\prime}(0)>0$, then the spectral flow is $S F_{\mathbb{C}}\left(\partial_{\nu_{t}}^{g_{t}}\right)=1$ on the path $t \in[-1,1]$. If we have $\gamma\left(Y, g_{0}, \nu_{0}\right)>0$, then there is a unique irreducible solution, which contributes a +1 to the invariant, that flows into the reducible as $t \rightarrow 0$, with $t<0$. If we have $\gamma\left(Y, g_{0}, \nu_{0}\right)<0$, then a unique irreducible that contributes a -1 to the invariant approaches the reducible as $t \rightarrow 0, t>0$. This gives the wall crossing term

$$
\lambda\left(Y, g_{1}\right)=\lambda\left(Y, g_{-1}\right)-S F_{\mathbb{C}}\left(\partial_{\nu_{t}}^{g_{t}}\right) .
$$

This provides a geometric interpretation of the wall-crossing formula that we derived algebraically in the previous section from the exact sequences.

Remark. With the metric dependence of $\lambda(Y, \mathbf{s}, g)$ understood, we can modify this quantity by introducing a correction term as follows. Choose any four manifold $X$ with boundary $Y$, such that $X$ is endowed with a cylindrical-end metric modeled on $(Y, g)$. Choose a $\operatorname{Spin}_{c}$ structure $\mathbf{s}_{X}$ on $X$, which over the end is the pullback of $\mathbf{s}$ on $Y$, and choose a connection $\mathbb{A}$ on $\left(X, \mathbf{s}_{X}\right)$, which extends the unique reducible $\theta_{\mathbf{s}}$ on $(Y, \mathbf{s})$. Then we set

$$
\xi_{Y}(\mathbf{s}, g)=\operatorname{Ind}_{\mathbb{C}}\left(D_{\mathbb{A}}^{X}\right)-\frac{1}{8}\left(c_{1}\left(\mathbf{s}_{X}\right)^{2}-\sigma(X)\right),
$$

where $\operatorname{Ind} d_{\mathbb{C}}\left(D_{\mathbb{A}}^{X}\right)$ is the complex index of the Dirac operator on $\left(X, \mathbf{s}_{X}\right)$, twisted with the chosen $\operatorname{Spin}_{c}$ connection $\mathbb{A}$, and $\sigma(X)$ is the signature of $X$. By the Atiyah-Patodi-Singer index theorem, $\xi_{Y}(\mathbf{s}, g)$ is independent of the choice of $\left(X, \mathbf{s}_{X}\right)$ and $\mathbb{A}$. Actually, $\xi_{Y}(\mathbf{s}, g)$ can be expressed as a combination of the Atiyah-Patodi-Singer eta invariants for the Dirac operator and signature operator on $(Y, \mathbf{s})$. From the definition (131), we see that

$$
\xi_{Y}\left(\mathbf{s}, g_{1}, \nu_{1}\right)-\xi_{Y}\left(\mathbf{s}, g_{-1}, \nu_{-1}\right)=-S F_{\mathbb{C}}\left(\partial_{\nu_{t}}^{g_{t}}\right),
$$

where $\left(g_{t}, \nu_{t}\right)$ is a family of metrics and imaginary-valued 1-forms on $Y$. Then the modified SW invariant

$$
\lambda(Y, g)-\xi_{Y}(\mathbf{s}, g)
$$

is a topological invariant of $(Y, \mathbf{s})$. In [43], we show that the averaged version of these modified Seiberg-Witten invariants agrees with the Casson-Walker invariant for any rational homology sphere. 


\section{Index.}

$\#, \S 4.2$, p. 514

$\hat{\#}, \S 4.2$, p. $514, \S 4.3$, p. 543

ApprCoker, §4.2, p. 527

ApprKer, $\S 4.2$, p. 527

$\mathcal{A}_{k}, \S 2.1$, p. 462

$\mathcal{A}_{k, \delta}\left(O_{a}, O_{b}\right), \S 3.2$, p. 491

$\mathcal{B}^{0}, \S 3.1$, p. $487, \S 4$, p. 51

$\mathcal{B}_{k \delta}^{0}\left(O_{a}, O_{b}\right), \S 3.2$, p. 491

$C_{*, U(1)}(Y), \S 5.1$, p. 550

$\mathcal{C}, \S 2.1$, p. 463

$\mathcal{C}_{\rho}, \S 2.1$, p. 466

$\partial_{\nu}^{g}, \S 2.1$, p. 467

$D, \S 5.1$, p. 550

$\nabla \mathcal{F}, \S 2.1$, p. 46

$F_{\# T}, \S 4.2$, p. 523

$\tilde{F}_{\#_{T}}, \S 4.2$, p. 534

$e_{a}^{+}, e_{b}^{-}, \S 3.2$, p. $491, \S 6$, p. 565,566

$e_{\delta}(t), \S 2.3$, p. 473

$\left.G\right|_{(A, \psi)}, \S 2.1$, p. 465

$G_{(A, \psi)}^{*}, \S 2.1$, p. 466

$\tilde{\mathcal{G}}_{Y \times \mathbb{R}}, \S 2.1$, p. 465

$\tilde{\mathcal{G}}_{k+1}, \S 2.1$, p. 462

$\mathcal{G}, \S 2.1$, p. 463

$\mathcal{G}^{0}, \S 3.1$, p. 487

$\mathcal{G}_{k+1, \delta}^{0}\left(O_{a}, O_{b}\right), \S 3.2$, p. 491

$H F_{*}^{S W}{ }_{U(1)}, \S 5.2$, p. 552

$H_{x \#_{T}^{0} y}^{0}, \S 4.2$, p. 525

$H_{x \#_{T}^{0} y}^{1}, \S 4.2$, p. 525

$H, \S 6$, p. 568

$I, \S 6$, p. 565
Ind $_{e}, \S 3.3$, p. 492

$J, \S 6$, p. 567

$\mathrm{Ker}_{e}, \S 3.3$, p. 492

$\left.L\right|_{(A, \psi)}, \S 2.1$, p. 465

$\mathcal{L}_{b}, \S 4.2$, p. 518

$\mathcal{L}_{x}, \S 2.4$, p. $477, \S 4.2$, p. 517

$\mathcal{L}_{x \#_{T}^{0} y}, \S 4.2$, p. 518

$\mu(a)-\mu(b), \S 2.4$, p. 478

$\mathcal{M}, \S 2.1$, p. 467

$\mathcal{M}^{0}, \S 3.1$, p. 487

$\mathcal{M}(a, b), \S 2.5$, p. 484

$\hat{\mathcal{M}}(a, b), \S 2.5$, p. 484

$\mathcal{M}\left(O_{a}, O_{b}\right), \S 3.2$, p. 491

$\mathcal{M}\left(O_{a}, O_{a^{\prime}}\right), \S 6$, p. 565

$\mathcal{M}\left(O_{a}, O_{a^{\prime}}\right)^{*}, \S 6$, p. 566

$\mathcal{M}^{P}\left(O_{a}, O_{b}\right), \S 6$, p. 567

$\mathcal{M}^{\text {bal }}\left(O_{a}, O_{b}\right), \S 4$, p. 503

$\hat{\mathcal{M}}\left(O_{a}, O_{b}\right), \S 4$, p. 502

$\hat{\mathcal{M}}\left(O_{a}, O_{b}\right)^{*}, \S 4$, p. 501

$m_{a c}^{P}, \S 6$, p. 570

$m_{a b^{\prime}}, \S 6$, p. 569

$n_{\theta_{0}, b}^{P}, \S 6$, p. 571

$n_{a, \theta_{0}}^{P}, \S 6$, p. 570

$n_{a b}^{P}, \S 6$, p. 570

$n_{\theta_{0} b^{\prime}}, \S 6$, p. 570

$n_{a \theta_{1}}, \S 6$, p. 569

$n_{a a^{\prime}}, \S 6$, p. 569

$\Omega_{* U(1)}\left(O_{a}\right), \S 5$, p. 550

$O_{a}, \S 3.2$, p. 490 
$P_{(\mathbb{A}, \Psi)}, \S 2.3$, p. 474

$\mathcal{D} P_{(\mathbb{A}, \Psi)}, \S 2.4$, p. 477

$\mathcal{P}, \S 2.3$, p. 474

$q_{(\mathbb{A}, \Psi)}, \S 2.3$, p. 474

$\sigma(\psi, \psi), \S 2.1$, p. 463

$\sigma(x, y, T), \S 4.2$, p. 532

$\sigma_{k}\left(x_{0}, \ldots, x_{k}, T_{1}, \ldots, T_{k}\right), \S 4.3$, p. 543

$S F\left(\partial_{\nu_{t}}^{g t}\right), \S 6$, p. $56, \S 7.2$, p. 622

$\mathcal{S}_{\Gamma_{a b}}, \S 4.2$, p. 513,515

$\bar{s}, \S 6.2$, p. 596 $\tilde{s}, \S 6.2$, p. 596

$s_{\mu}, \S 6.2$, p. 591

$\theta=[\nu, 0], \S 2.1$, p. 467

$\left.T\right|_{(A, \psi)}, \S 2.1$, p. 466

$\mathcal{T}_{x \#_{T}^{0} y}, \S 4.2$, p. 530

$\mathcal{U}\left(O_{a}, O_{c}\right), \S 4.2$, p. 530

$V_{\Gamma}, \S 4.2$, p. 513

$W_{\Gamma}^{ \pm}, \S 4.2$, p. 513

$\mathcal{W}_{n}, \S 2.2$, p. 471

$x \#_{T}^{0} y, \S 4.2$, p. 516 


\section{References.}

[1] M.F. Atiyah, New invariants of 3- and 4-dimensional manifolds, Symp. Pure Math., 48 (1988), 285-299.

[2] M.F. Atiyah and R. Bott, The moment map and equivariant cohomology, Topology, 23 (1984), 1-28.

[3] M.F. Atiyah, V.K. Patodi and I.M. Singer, Spectral asymmetry and Riemannian geometry, I,II,III; Math. Proc. Cambridge Phil. Soc., 77 (1975), 43-69; 78 (1975), 405-432; 79 (1976), 71-99.

[4] D. Auckly, Surgery, knots and the Seiberg-Witten equations, Lectures for the 1995 TGRCIW, preprint.

[5] D. Auckly, The Thurston norm and three-dimensional Seiberg-Witten theory, Osaka J. Math., 33(3) (1996), 737-750.

[6] D.M. Austin and P.J. Braam, Equivariant Floer theory and gluing Donaldson polynomials, Topology, 35(1) (1996), 167-200.

[7] D.M. Austin and P.J. Braam, Morse-Bott theory and equivariant cohomology, Floer Memorial Volume, Birkhäuser, 1995.

[8] D.M. Austin and P.J. Braam, Equivariant homology, Math. Proc. Camb. Phil. Soc., 118 (1995) 125-139.

[9] C. Bär, Metrics with harmonic spinors, Geom. Funct. Anal., 6(6) (1996), 899-942.

[10] S.E. Cappell, R. Lee and E.Y. Miller, Self-adjoint elliptic operators and manifold decompositions. I: Low eigenmodes and stretching, Commun. Pure Appl. Math., 49(8) (1996), 825-866.

[11] A.L. Carey, M. Marcolli and B.L. Wang, Exact triangles in SeibergWitten Floer theory, Part I: the geometric triangle, preprint.

[12] A.L. Carey, J. McCarthy, B.L. Wang and R.B. Zhang, Seiberg-Witten monopoles in three dimensions, Lett. Math. Phys., 39(3) (1997), 213228.

[13] A.L. Carey and B.L. Wang, Seiberg-Witten-Floer homology and Gluing formulae, preprint. 
[14] W. Chen, Casson's invariant and Seiberg-Witten gauge theory, Turkish J. Math., 21(1) (1997), 61-81.

[15] S.K. Donaldson, The Seiberg-Witten equations and 4-manifold topology, Bull. AMS, 33(1) (1996), 45-70.

[16] S.K. Donaldson, Connections, cohomology and the intersection forms of 4-manifolds, J. Differential Geom., 24(3) (1986), 275-341.

[17] S.K. Donaldson, The orientation of Yang-Mills moduli spaces and 4manifold topology, J. Differential Geom.,26 (1987), 397-428.

[18] S.K. Donaldson and P.B. Kronheimer, The geometry of four-manifolds, Oxford, 1991.

[19] M. Duflo and M. Vergne, Cohomologie équivariante de descente, Astérisque, 215 (1993), 5-108.

[20] J. Eichhorn, Elliptic differential operators on noncompact manifolds, Seminar Analysis of the Karl-Weierstrass-Institute of Mathematics, 1986/87 (Berlin, 1986/87), 4-169, Teubner-Texte Math., 106, Teubner, Leipzig, 1988.

[21] P.M.N. Feehan, Generic metrics, irreducible rank-one PU(2) monopoles, and transversality, preprint, math.DG/9809001.

[22] P.M.N. Feehan and T.G. Leness, Donaldson invariants and wall-crossing formulas. I: Continuity of gluing maps, preprint, math.DG/9812060.

[23] R. Fintushel and R. Stern, Integer graded instanton homology groups for homology three-spheres, Topology, 31(3) (1992), 589-604.

[24] A. Floer, An instanton-invariant for 3-manifolds, Comm. Math. Phys., 118 (1988), 215-240.

[25] K.A. Froyshov, The Seiberg-Witten equations and four-manifolds with boundary, Math. Res. Lett., 3(3) (1996), 373-390.

[26] N. Hitchin, Harmonic spinors, Advances in Mathematics, 14 (1974), $1-54$.

[27] K. Iga, Moduli spaces of Seiberg-Witten flows, Ph.D. thesis, Stanford, June 1998. 
[28] U. Koschorke, Infinite-dimensional K-theory and characteristic classes of Fredholm bundle maps, 1970 Global Analysis (Proc. Sympos. Pure Math., Vol. XV, Berkeley, Calif., 1968), 95-133, Amer. Math. Soc.

[29] P.B. Kronheimer, Embedded surfaces and gauge theory in three and four dimensions, in 'Surveys in differential geometry', Vol. III (Cambridge, MA, 1996), 243-298, Int. Press, Boston, MA, 1998.

[30] P.B. Kronheimer and T.S. Mrowka, The genus of embedded surfaces in the projective plane, Math. Research Lett., 1 (1994), 797-808.

[31] P.B. Kronheimer and T.S. Mrowka, Monopoles and contact structures, Invent. Math., 130(2) (1997), 209-255.

[32] S. Kumar and M. Vergne, Equivariant cohomology with generalized coefficients, Astérisque, 215 (1993), 109-204.

[33] H.B. Lawson and M.L. Michelsohn, Spin geometry, Princeton 1989.

[34] W. Li, Künneth formulae and cross products for the symplectic Floer cohomology, Topology Appl., 110(3) (2001), 211-236.

[35] Y. Lim, Seiberg-Witten invariants for 3-manifolds in the case $b_{1}=0$ or 1, Pacific J. Math., 195(1) (2000), 179-204.

[36] R.B. Lockhard and R.C. McOwen, Elliptic operators on non-compact manifolds, Ann. Sci. Norm. Sup. Pisa, IV(12) (1985), 409-446.

[37] S. Maier, Generic metrics and connections on Spin- and Spin ${ }^{c}$ manifolds, Comm. Math. Phys., 188(2) (1997), 407-437.

[38] M. Marcolli, Seiberg-Witten-Floer homology and Heegaard splittings, Internat. J. Math., 7(5) (1996), 671-696.

[39] M. Marcolli, Seiberg-Witten gauge theory, Texts and Readings in Mathematics, 17, Hindustan Book Agency, New Delhi, 1999.

[40] M. Marcolli and B.L. Wang, Exact triangles in Seiberg-Witten Floer theory, Part II: geometric limits of flow lines, preprint.

[41] M. Marcolli and B.L. Wang, Exact triangles in Seiberg-Witten Floer theory, Part III: proof of exactness, preprint.

[42] M. Marcolli and B.L. Wang, Exact triangles in Seiberg-Witten Floer theory, Part IV: $\mathbb{Z}$-graded monopole homology, preprint. 
[43] M. Marcolli and B.L. Wang, Seiberg-Witten invariant and CassonWalker invariant for any rational homology 3-sphere, preprint.

[44] R.B. Melrose, Differential analysis on manifolds with corners, unpublished manuscript (available at the author's website.)

[45] J.W. Morgan, The Seiberg-Witten equations and applications to the topology of smooth four-manifolds, Princeton University Press, 1996.

[46] J.W. Morgan and T. Mrowka, On the gluing theorem for instantons on manifolds containing long cylinders, preprint.

[47] J.W. Morgan, T. Mrowka and D. Ruberman, The $L^{2}$-moduli space and a vanishing theorem for Donaldson polynomial invariants, International Press, 1994.

[48] J.W. Morgan, Z. Szabó and C.H. Taubes, A product formula for SeibergWitten invariants and the generalized Thom conjecture, J. Differential Geom., 44(4) (1996), 706-788.

[49] T.S. Mrowka, A local Mayer-Vietoris principle for. Yang-Mills moduli spaces, PhD thesis. U.C. Berkeley, 1989.

[50] T. Mrowka, P.Ozsváth and B. Yu, Seiberg-Witten monopoles on Seifert fibered spaces, Comm. Anal. Geom., 5(4) (1997), 685-791.

[51] L. Nicolaescu, private communication.

[52] L. Nicolaescu, Eta invariants of Dirac operators on circle bundles over Riemann surfaces and virtual dimensions of finite energy SeibergWitten moduli spaces, Israel J. Math., 114 (1999), 61-123.

[53] L. Nicolaescu, Generalized symplectic geometries and the index of families of elliptic problems, Memoirs AMS, 609 (1997).

[54] L. Nicolaescu, Adiabatic limits of the Seiberg-Witten equations on Seifert manifolds, Comm. Anal. Geom., 6(2) (1998), 331-392.

[55] Ch. Okonek and A. Teleman, 3-dimensional Seiberg-Witten invariants and non-Kählerian geometry, Math. Ann., 312(2) (1998), 261-288.

[56] D. Salamon, Spin geometry and Seiberg-Witten invariants, preprint.

[57] M. Schwarz, Morse Homology, Birkhäuser 1993. 
[58] L. Simon, Asymptotics for a class of non-linear evolution equations, with applications to geometric problems, Ann. of Math., 118 (1983), 525-571.

[59] C.H. Taubes, Self-dual connections on 4-manifolds with indefinite intersection matrix, J.Diff.Geom., 19 (1984), 517-560.

[60] C.H. Taubes, The stable topology of self-dual moduli spaces, J. Diff. Geom., 29 (1989), 163-230.

[61] C.H. Taubes, $L^{2}$-moduli spaces on 4-manifolds with cylindrical ends, International Press, 1993.

[62] C.H. Taubes, A simplicial model for Donaldson-Floer theory, preprint.

[63] B.L. Wang, Seiberg-Witten-Floer theory for homology 3-spheres, preprint, dg-ga/9602003.

[64] R.G. Wang, On Seiberg-Witten Floer invariants and the generalized Thom problem, preprint.

[65] G. Wang and R. Ye, Equivariant and Bott-type Seiberg-Witten Floer homology, I, II, preprints, math.DG/9901058 and math.DG/9901059.

[66] E. Witten, Monopoles and four-manifolds, Math. Research Lett., 1 (1994), 769-796.

MaX-Planck-INSitut Für Mathematik

BONN D-53111, GERMANY

E-mail address: marcolli@mpim-bonn.mpg.de

Department of Pure Mathematics,

The University of AdELAide

Adelaide SA 5005, Australia

E-mail address: bwang@maths.adelaide.edu.au

Received June 13, 1997. 